\title{
Computation of Complex Eigenmodes for Resonators Filled With Gyrotropic Materials
}

\section{Berechnung komplexer Eigenmoden von Resonatoren mit gyrotropen Materialien}

Vom Fachbereich Elektrotechnik und Informationstechnik der Technischen Universität Darmstadt zur Erlangung des akademischen Grades Doktor-Ingenieur (Dr.-Ing.)

genehmigte Dissertation von Klaus Klopfer, M. Sc. hon., aus Hammelburg

Tag der Einreichung: 01. Juli 2014, Tag der Prüfung: 15. Oktober 2014

Darmstadt 2014 - D 17

1. Gutachten: Prof. Dr.-Ing. Thomas Weiland

2. Gutachten: Prof. Dr.-Ing. Harald Klingbeil

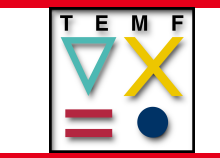


Computation of Complex Eigenmodes for Resonators Filled With Gyrotropic Materials Berechnung komplexer Eigenmoden von Resonatoren mit gyrotropen Materialien

Genehmigte Dissertation von Klaus Klopfer, M. Sc. hon., aus Hammelburg

1. Gutachten: Prof. Dr.-Ing. Thomas Weiland

2. Gutachten: Prof. Dr.-Ing. Harald Klingbeil

Tag der Einreichung: 01. Juli 2014

Tag der Prüfung: 15. Oktober 2014

Darmstadt - D 17

Bitte zitieren Sie dieses Dokument als:

URN: urn:nbn:de:tuda-tuprints-42100

URL: http://tuprints.ulb.tu-darmstadt.de/4210

Dieses Dokument wird bereitgestellt von tuprints,

E-Publishing-Service der TU Darmstadt

http://tuprints.ulb.tu-darmstadt.de

tuprints@ulb.tu-darmstadt.de

\section{(c) $(1) \Theta \Theta$ \\ BY NC ND}

Die Veröffentlichung steht unter folgender Creative Commons Lizenz:

Namensnennung - Keine kommerzielle Nutzung - Keine Bearbeitung 2.0 Deutschland http://creativecommons.org/licenses/by-nc-nd/2.0/de/ 


\section{Zusammenfassung}

In der vorliegenden Arbeit wird die numerische Berechnung von komplexen Eigenmoden von Resonatoren mit gyrotropem Material behandelt. Zu diesem Zweck wurde ein geeigneter Löser auf Grundlage der Methode der Finiten Integration (FIT) entwickelt, effizient implementiert und erfolgreich verifiziert.

Eigenwertprobleme mit gyrotropen Materialien treten beispielsweise bei der Berechnung der Resonanzfrequenzen von ferritgeladenen Resonatoren wie der GSI SIS 18 Kavität auf. Ferrite weisen gyromagnetisches Verhalten auf. Dieses zeichnet sich durch eine anisotrope Permeabilität aus, die zudem von der Frequenz des Wechselfeldes wie auch von der Vormagnetisierung abhängt. Analog kann gyroelektrisches Material, wie zum Beispiel magnetisiertes Plasma, durch einen frequenz- und vormagnetisierungsabhängigen Permittivitätstensor beschrieben werden. Da diese Materialtensoren in die Systemmatrix des Eigenwertproblems eingehen, wird ein geeigneter Löser benötigt. Im Rahmen dieser Arbeit wird FIT auf einem hexaedrischen ,staircase $^{\natural}$ Gitter zur Diskretisierung verwendet. In der Standardformulierung ist FIT jedoch auf diagonal anisotrope Materialien beschränkt. Ein wesentliches Ziel dieser Arbeit war es daher, die Methode auf gyromagnetische und gyroelektrische Substanzen im Frequenzbereich zu erweitern. Die hergeleiteten Ausdrücke für die nichtdiagonalen Materialmatrizen sind für den Fall nichtgyrotroper Substanzen konsistent mit der Standardformulierung. Darüber hinaus weisen sie im verlustlosen Fall selbst für nichtäquidistante Gitter eine hermitesche Struktur auf.

Auf Grund der genannten Anforderungen ist der neu entwickelte Löser aus zwei Teilkomponenten aufgebaut: Die erste ist ein Magnetostatiklöser für nichtlineares Material basierend auf der $H_{i}$-Methode zur Berechnung des Magnetfeldes, welches durch den Vormagnetisierungsstrom hervorgerufen wird. Mit Hilfe der berechneten Feldverteilung werden anschließend die Materialeigenschaften am Arbeitspunkt lokal in jeder Gitterzelle ausgewertet. Die zweite Komponente ist ein Löser vom Jacobi-Davidson Typ zur iterativen Lösung des nichtlinearen Eigenwertproblems. Um Materialverluste berücksichtigen zu können, werden vom Eigenwertlöser auch Systemmatrizen mit nichthermitescher Struktur unterstützt. Außerdem wird eine effiziente parallele Rechnung auf Maschinen mit verteiltem Speicher ermöglicht. Hierzu ist eine vom Standardschema abweichende Anordnung der FIT Freiheitsgrade implementiert, wodurch ein höheres Verhältnis von Rechen- zu Kommunikationszeit resultiert. Weiterhin werden alle Freiheitsgrade, die aus verschiedenen Gründen a priori verschwinden, vollständig aus allen Vektoren und Matrizen entfernt. Insgesamt können so gyrotropische Eigenwertprobleme mit einer Anzahl von mehreren Millionen Gitterzellen in einer vertretbaren Zeit gelöst werden. Die Gültigkeit der numerischen Ergebnisse wird anhand von (semi-)analytischen Vergleichsrechnungen bestätigt.

Als Anwendungsbeispiel wird eine Eigenmodeanalyse der GSI SIS 18 Kavität durchgeführt. Da keine ausreichenden Materialdaten aus dem Datenblatt des Herstellers verfügbar sind, wurden Messungen der benötigten magnetischen Kennzahlen des Ferroxcube 8C12m Ferrits durchgeführt. Ringe aus diesem Material sind in der GSI SIS 18 Kavität eingebaut. Zu den gemessenen Größen zählen die komplexe Permeabilität als Funktion von Frequenz und Vormagnetisierungsfeld bei niedrigem Pegel sowie die $B-H$-Kurve. Die verwendeten Messmethoden werden ebenso wie die detaillierte Analyse zusammen mit den ausgewerteten Daten vorgestellt. Die so erhalte- 
ne skalare Permeabilität wird dann in der Simulation verwendet. Die berechneten Werte für die Resonanzfrequenz und des Qualitätsfaktors der Grundmode sind mit den vorliegenden Messdaten vereinbar. Um das weitere Potential des Lösers aufzuzeigen, wird zusätzlich eine Analyse von Moden höherer Ordnung durchgeführt sowie ein Ausblick zur Untersuchung von Vormagnetisierungsschemen mit sich senkrecht überlagernden Magnetfeldern gegeben. 


\section{Abstract}

In this thesis, the numerical computation of complex eigenmodes of cavity resonators filled with magnetically biased gyrotropic material is demonstrated. For this purpose, a dedicated solver based on the Finite Integration Technique (FIT) has been developed, efficiently implemented as well as successfully verified.

Gyrotropic field problems arise, for instance, for the calculation of the resonance frequencies of ferrite-loaded resonators like the GSI SIS 18 cavity. Ferrites exhibit gyromagnetic properties with an anisotropic permeability, which furthermore depends both on frequency and bias field. Similarly, gyroelectric material such as magnetized plasmas can be described by a frequencyand bias field dependent permittivity tensor. Since these material tensors affect the system matrix of the eigenvalue problem, a dedicated solver is required. In this thesis, the FIT with a hexaedral staircase filling is employed for discretization. In the standard formulation, it is, however, limited to diagonally anisotropic materials. Hence, as one of the goals of this thesis, the FIT has been extended to gyromagnetic as well as gyroelectric materials in frequency domain. The derived expressions for the non-diagonal material matrices are fully consistent with the standard FIT when applied to non-gyrotropic materials. Moreover, their structure is manifestly Hermitian in the lossless case, even for non-equidistant grids.

Due to the above-mentioned material requirements, the newly developed solver consists of two components: The first one is a magnetostatic solver based on the $H_{i}$-algorithm supporting nonlinear material to calculate the magnetic field excited by the bias current. Having obtained the field distribution, the material properties are evaluated locally in each mesh cell at the specified working point. The second component is a Jacobi-Davidson type eigenvalue solver for the iterative solution of the nonlinear eigenproblem. To be capable of handling material losses, the eigensolver also supports non-Hermitian eigenproblems. What is more, efficient parallel computing on machines with distributed memory is possible. To this end, an ordering of the FIT-DOFs different from the standard scheme is implemented, which results in an increased computation to communication ratio. Furthermore, all DOFs that vanish a priori due to several reasons are completely removed from the vectors and matrices. All in all, gyrotropic eigenproblems discretized with several millions of mesh cells can be solved in a reasonable time by the developed solver. The validity of the numerically obtained results is confirmed by thorough comparisons with (semi-)analytical calculations.

As an application example, an eigenmode analysis of the GSI SIS 18 cavity is carried out. Since the required material data are not available in the data sheet of the manufacturer, designated measurements of the magnetic characteristics of the Ferroxcube $8 \mathrm{C} 12 \mathrm{~m}$ ferrite ring cores, which are installed inside the GSI SIS 18 cavities, were performed. Among these characteristics are the complex permeability as a function of frequency and bias magnetic field strength at low radiofrequency power levels as well as the $B-H$ curve. The measurement methods together with the detailed data analysis including the presentation of evaluated data are supplemented to this thesis. The scalar, isotropic permeability retrieved this way is used for the cavity simulations. The obtained values for the resonance frequency and quality factor for the fundamental mode are in accordance with available measurement data. To demonstrate the further potential of 
the solver, also higher-order modes are investigated and an outlook on possibly advantageous 2-directional bias schemes is given. 


\section{Contents}

1. Introduction 1

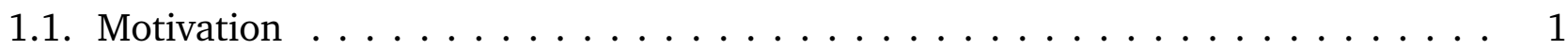

1.1.1. GSI SIS 18 ferrite cavity $\ldots \ldots \ldots \ldots \ldots \ldots \ldots \ldots \ldots \ldots \ldots \ldots \ldots$

1.1.2. Facility for Antiproton and Ion Research (FAIR) $\ldots \ldots \ldots \ldots \ldots$

1.2. Overview ........................... 3

2. Material modeling and measurement of ferrites 5

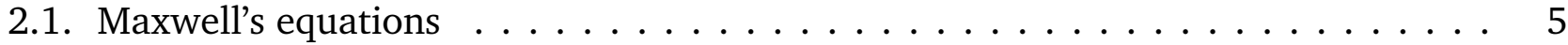

2.2. Fundamental material properties $\ldots \ldots \ldots \ldots \ldots$

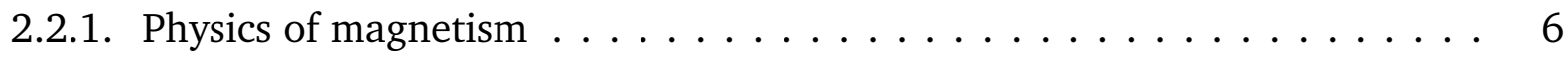

2.2.2. Magnetodynamics equation $\ldots \ldots \ldots \ldots \ldots \ldots$

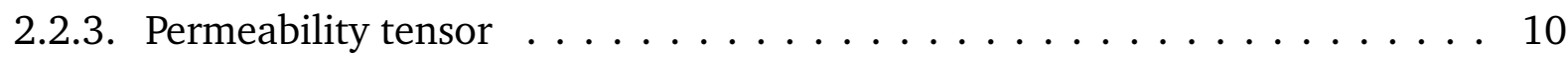

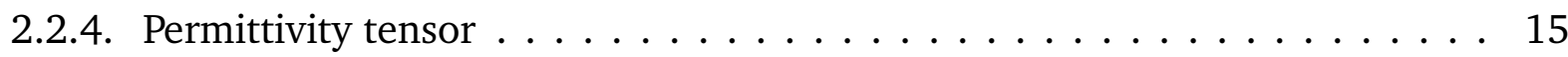

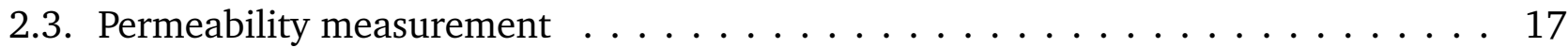

2.3.1. Measurement setup . . . . . . . . . . . . . . . . . . 17

2.3.2. Analysis of the reflection measurement data . . . . . . . . . . . . . . 19

2.3.3. Uncertainty estimation for the reflection measurement . . . . . . . . . 26

2.3.4. Analysis of the transmission measurement data . . . . . . . . . . . . . . 28

2.3.5. Uncertainty estimation for the transmission measurement . . . . . . . 30

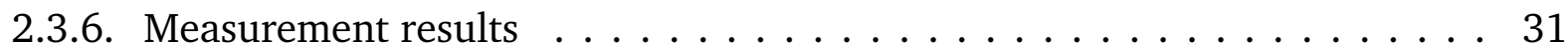

2.3.7. Measurement of the $B-H$ curve of a working cycle with reduced bias range 36

3. Calculation of eigensystems for materials with fully occupied material tensors 39

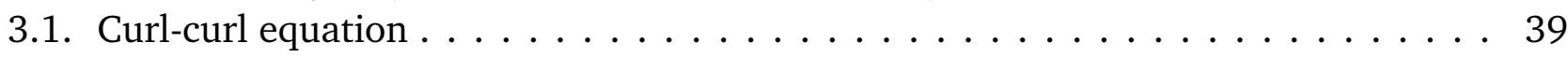

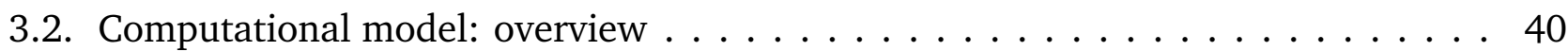

3.3. Basics principles of distributed computing . . . . . . . . . . . . . 41

3.4. The Finite Integration Technique . . . . . . . . . . . . . . . . . . . 42

3.4.1. Definition of the primary and dual grid . . . . . . . . . . . . 42

3.4.2. Grid allocation of degrees of freedom . . . . . . . . . . . . 43

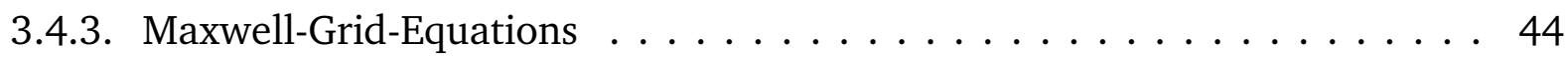

3.4.4. Construction of the material matrices . . . . . . . . . . . . . 45

3.4.5. Discretized matrices . . . . . . . . . . . . . . . . . 48

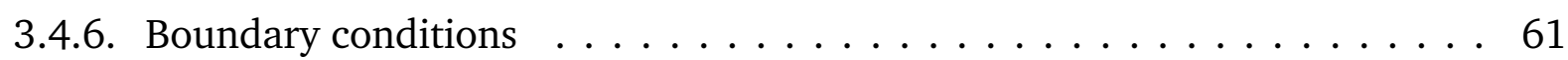

3.4.7. Reduced grid . . . . . . . . . . . . . . . . . . . 61

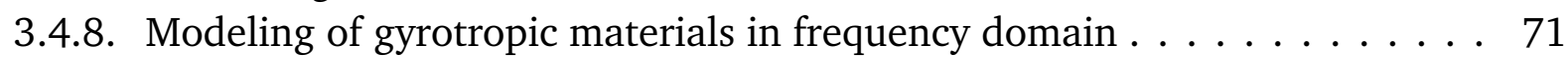

3.4.9. Curl-curl equation in the FIT . . . . . . . . . . . . . 78

3.4.10. Lumped elements . . . . . . . . . . . . . . . . . 78

3.4.11. Magnetostatic field problem . . . . . . . . . . . . . . 80 
3.5. Numerical methods for the computation of eigenvectors . . . . . . . . . . . . . 89

3.5.1. General remarks . . . . . . . . . . . . . . . . . . 89

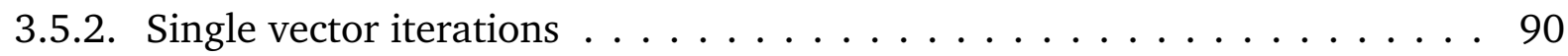

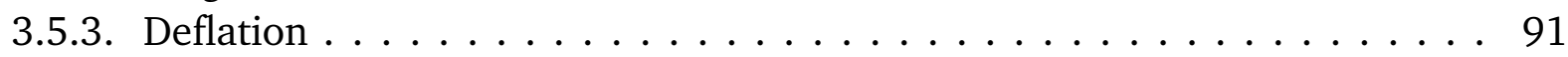

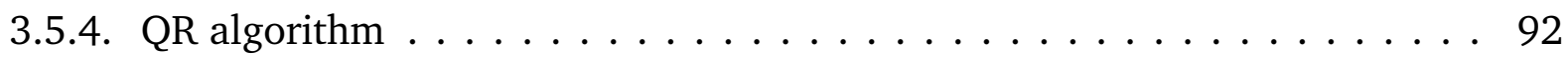

3.5.5. Subspace methods with projection . . . . . . . . . . . . . . 93

3.5.6. Implementation of the eigenvalue solver $\ldots \ldots \ldots \ldots 2$

4. Applications 111

4.1. Prerequisites for the material modeling . . . . . . . . . . . . . . . . . 111

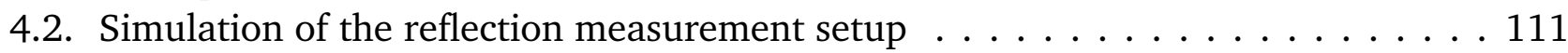

4.3. Simulation of the GSI SIS 18 ferrite cavity . . . . . . . . . . . . . . . . 114

4.3.1. Analysis of the fundamental mode . . . . . . . . . . . . . . . . . 114

4.3.2. Comparison with nonlinear equivalent circuit model . . . . . . . . . . . 117

4.3.3. Analysis of higher-order modes . . . . . . . . . . . . . . . . . . . . . . . . . . . . . . . .

4.3.4. 2-directional bias scheme . . . . . . . . . . . . . . . . . 119

$\begin{array}{ll}\text { 5. Summary and outlook } & 121\end{array}$

$\begin{array}{ll}\text { A. Proofs } & 123\end{array}$

A.1. Proof of the properties of the topological matrices for arbitrary ordering schemes 123

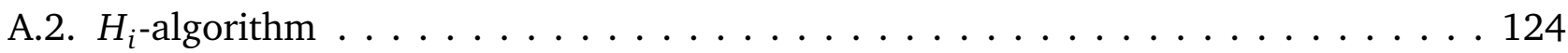

A.2.1. Initialization of the $H_{i}$-components on the surface $\ldots \ldots \ldots \ldots 124$

A.2.2. Determination of the $H_{i}$-components after each subdivision $\ldots \ldots \ldots \ldots$

B. Simplified class diagram of the combined magnetostatic and eigenvalue solver 129

$\begin{array}{ll}\text { List of abbreviations and symbols } & 131\end{array}$

$\begin{array}{ll}\text { Bibliography } & 143\end{array}$ 


\section{Introduction}

\subsection{Motivation}

\subsubsection{GSI SIS 18 ferrite cavity}

The GSI Helmholtzzentrum für Schwerionenforschung in Darmstadt [1] operates the heavy-ion synchrotron SIS 18 for fundamental research. Two identical cavities are installed within the ring for the acceleration of the heavy ions. On each side of the accelerating gap, 32 ring cores of the Ferroxcube $8 \mathrm{C} 12 \mathrm{~m}$ ferrite material are installed, which can be magnetically biased parallel to the superimposed radio-frequency (RF) by means of six crossed (figure-of-eight) current windings (cf. Fig. 1.1(a)). The main advantage of such ferrite cavities for the acceleration of heavy ions in ramped operation in synchrotrons is twofold [2]: On the one hand, the high permeability of the ferrite material causes a reduction of the wavelength compared to vacuum. This allows the construction of smaller accelerating structures, which is beneficial in the range of relatively low resonance frequencies in the order of a few $\mathrm{MHz}$. On the other hand, the dependence of the incremental permeability on the bias magnetic field strength enables to quickly modify the eigenfrequency of the resonator system (cf. Fig. 1.1(b)). The tuning is particularly important during the acceleration phase. Here the resonance frequency of the cavities has to be adjusted to the revolution frequency of the heavy ions to reflect their increasing speed. For the SIS 18 ferrite cavity, biasing enables to alter the resonance frequency in a range of about 0.6 to $5.0 \mathrm{MHz}$ by driving the bias current from $\approx 5 \mathrm{~A}$ up to $\approx 500 \mathrm{~A}[2]$. A more detailed description of the GSI SIS 18 ferrite cavity can be found in [2].

\subsubsection{Facility for Antiproton and Ion Research (FAIR)}

Though the SIS 18 synchrotron has been operated since the year 1990, new interest in a better understanding of the SIS 18 cavity, particularly of the tuning process, has aroused recently. The main reason is the planning of the new international Facility for Antiproton and Ion Research (FAIR) [3], which is currently under construction at the GSI site. It is expected that FAIR will provide unique opportunities for fundamental research in a variety of fields, including nuclear, atomic, anti-matter and plasma physics as well as biology and bio-medical science. A schematic sketch of the planned facility is shown in figure 1.2. One of the main components will be the double ring synchrotron SIS $100 / 300$ with a circumference of $1.1 \mathrm{~km}$. A proper understanding of the GSI SIS 18 cavity is crucial for a successful operation of the FAIR synchrotron for two reasons: Firstly, the SIS 18 ring will be used as injector for SIS $100 / 300$. Thus, the provided beam has to fulfill the FAIR specification. Secondly, it is foreseen that several accelerating cavities of the SIS 18 type will also be installed in the SIS 100 synchrotron. For that reason, it is the aim of the presented thesis to develop a tool for the numerical computation of the lowest eigenmodes of biased, ferrite-loaded resonators. Moreover, relevant material characteristics 
(a)

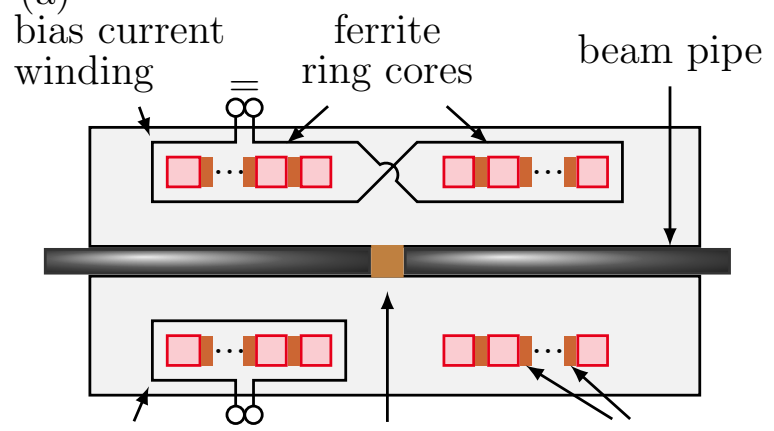

RF winding $\sim$ ceramic gap copper (cooling) (b)

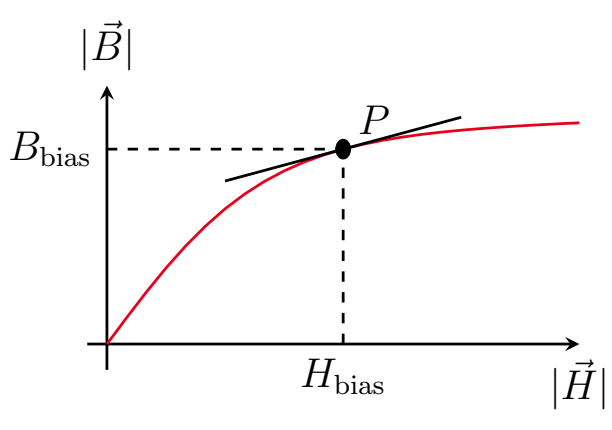

Figure 1.1.: (a) Simplified sketch of the main components of the GSI SIS 18 ferrite cavity. (b) Typical dependence of the magnetic induction on the magnetic field strength (neglecting hysteresis). The incremental permeability at the working point $P$ changes with the bias field strength $H_{\text {bias }}$.

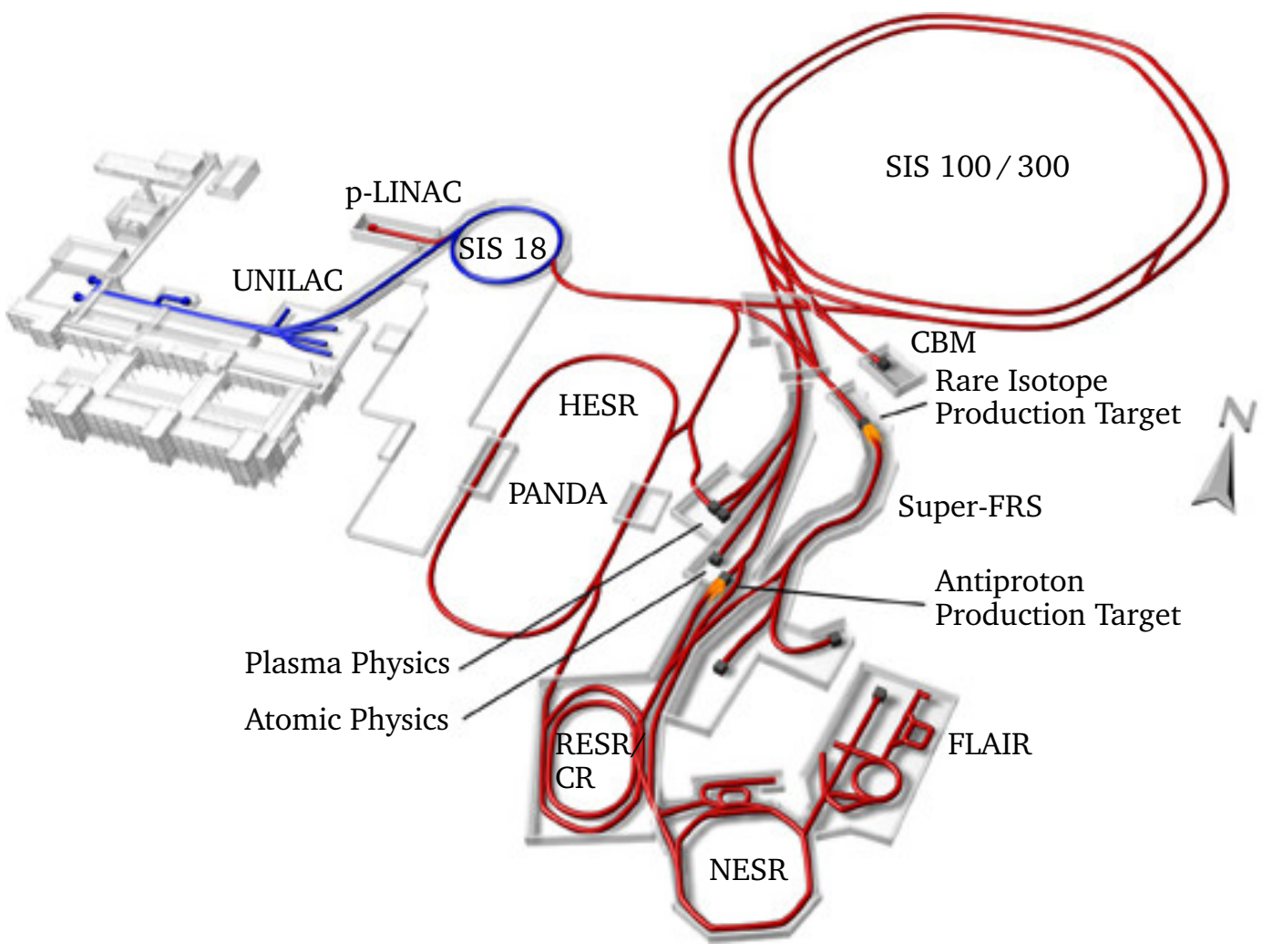

Figure 1.2.: Schematic sketch of the FAIR complex. The planned infrastructure for FAIR is shown in red, the already existing one of the GSI facility in blue. [3] 
are required for the simulations. Since, however, only limited information is provided by the manufacturer in the data sheet, designated measurements were carried out at the GSI facility. Hence, one part of the thesis is also dedicated to the measurement and data analysis of the magnetic properties of the Ferroxcube $8 \mathrm{C} 12 \mathrm{~m}$ material.

\subsection{Overview}

This thesis is structured as follows:

In chapter 2, fundamental properties of gyrotropic materials are discussed. The emphasis is clearly put on the magnetic characteristics of ferrites and other gyromagnetic substances. Accordingly, the structure of the permeability tensor is treated in detail. Furthermore, section 2.3 is dedicated to the permeability measurements, which were carried out at the GSI facility as part of this thesis. After the description of the measurement methods, obtained results for the complex permeability as a function of frequency and bias current as well as for the $B-H$ curve are presented.

After that, the problem of the numerical calculation of eigensystems for materials with gyrotropic properties is addressed in chapter 3. At first, the requirements on the applied computational model are elaborated, also taking into account aspects relevant for efficient distributed computing. The employed method for the numerical solution of the electromagnetic field problems, in this work the Finite Integration Technique, is introduced subsequently. This includes a detailed discussion of the structure of the involved topological matrices for different ordering schemes of the DOFs and the extension of the standard method to materials characterized by fully occupied material tensors in frequency domain. The properties of the gyrotropic materials finally lead to complex nonlinear eigenvalue problems. Hence, numerical methods for their solution are reviewed in section 3.5. Moreover, implementation details and numerical examples for verification are provided in the particular sections of that chapter.

In chapter 4 , the developed solver is used for the simulation of the measurement setup mentioned in chapter 2 as well as for the simulation of the GSI SIS 18 ferrite cavity. For the latter, both the fundamental and higher-order modes are analyzed and an outlook on 2-directional bias schemes is given.

The thesis closes with a summary and outlook. 


\section{Material modeling and measurement of ferrites}

In this chapter, the material modeling of ferrites as well as the measurement of magnetic material characteristics required for the eigenmode computation of ferrite-loaded devices is discussed. To begin with, Maxwell's equations are stated for further reference. In section 2.2, an overview of fundamental material properties of ferrites is given, which particularly focuses on the dynamic response of a ferrite excited by an electromagnetic RF-field for different magnetized states. Additionally, the permittivity tensor of gyroelectric materials is presented. Section 2.3 deals with the measurements that were performed at the GSI facility and their analysis.

\subsection{Maxwell's equations}

Maxwell's equations are the basis for the description of phenomena of electromagnetism. In differential form, the macroscopic variant takes the form [4]:

$$
\begin{aligned}
\nabla \times \vec{E} & =-\frac{\partial \vec{B}}{\partial t}, \\
\nabla \times \vec{H} & =\vec{J}+\frac{\partial \vec{D}}{\partial t}, \\
\nabla \cdot \vec{D} & =\rho, \\
\nabla \cdot \vec{B} & =0 .
\end{aligned}
$$

Therein, $\vec{E}$ is the electric field, $\vec{D}$ the electric displacement, $\vec{B}$ the magnetic induction (or magnetic flux density) and $\vec{H}$ the magnetizing field. Moreover, the total density of free charges is denoted by $\rho$ and the (free) current density by $\vec{J}$. All of the quantities are implicitly assumed to be dependent on position and time. The electric and magnetic fields are related to each other by [4]

$$
\begin{aligned}
& \vec{D}=\varepsilon_{0} \vec{E}+\vec{P}, \\
& \vec{B}=\mu_{0} \vec{H}+\mu_{0} \vec{M},
\end{aligned}
$$

with the polarization field $\vec{P}$, the magnetization field $\vec{M}$ and the permittivity and permeability of free space $\varepsilon_{0}$ and $\mu_{0}$, respectively. Only in the special case of linear, isotropic materials the constitutive relations simplify to

$$
\begin{aligned}
\vec{D} & =\varepsilon_{0} \vec{E}+\varepsilon_{0} \chi_{e} \vec{E}, \\
\vec{B} & =\mu_{0} \vec{H}+\mu_{0} \chi_{m} \vec{H},
\end{aligned}
$$

with the material specific electric and magnetic susceptibilities $\chi_{e}$ and $\chi_{m}$, respectively. In the general case, however, the fields $\vec{P}$ and $\vec{M}$ may be complicated functions of both $\vec{E}$ and $\vec{B}$. In the following section, those relations are discussed for magnetized ferrites as well as magnetized plasmas. 


\subsection{Fundamental material properties}

An overview over fundamental magnetic material properties is given in this section. After briefly reviewing some basics on the physics of magnetism, the magnetodynamics equation taking into account different phenomenological loss terms is stated. From this equation, the form of the permeability tensor for the fully magnetized state is derived and expressions for more general cases are given in subsection 2.2.3. For completeness, also the permittivity tensor as suitable for the description of the electric characteristics of magnetized plasmas is discussed in subsection 2.2.4.

\subsubsection{Physics of magnetism}

The macroscopic magnetization of solids is composed of the total of all microscopic magnetic moments of the constituents of the solid. Depending on the type of the individual magnetic moments of the constitutive atoms as well as on the interaction between them, different magnetic phenomena are observed. Accordingly, magnetism may be classified into the following types $[5,6]$.

\subsubsection{Diamagnetism}

Purely diamagnetic substances do not have a net resulting magnetic moment. Yet, an external magnetic field induces magnetic moments in its opposite orientation, which act against the external field. Thus, their magnetic susceptibility $\chi_{m}$ is negative and typically in the order of $-10^{-4}$ to $-10^{-5}$. Though the diamagnetic effect is intrinsic to all materials, it may be overcompensated by stronger paramagnetic contributions.

\subsubsection{Paramagnetism}

Paramagnetic effects occur in materials which are constituted by atoms featuring a net magnetic moment independent of the applied field [6]. Due to thermal excitation, the individual atomic magnetic moments are oriented randomly in the absence of an external field. With increasing field strength, the magnetic moments gradually line up with the applied field resulting in a positive macroscopic magnetization in the direction of the external field (cf. Fig. 2.1). Hence, the magnetic susceptibility is positive and typically in the order of $10^{-5}$ to $10^{-3}$.

\subsubsection{Ferromagnetism}

Also the constitutive atoms of ferromagnetic materials exhibit a net magnetic moment, which is due to the spin of single shell-electrons. In contrast to paramagnetism, a spontaneous magnetization is observed, that is, an alignment of the magnetic moments in parallel in the absence of an applied field [6]. Such behavior can only be explained with the help of a quantum mechanical effect, the exchange interaction. If the temperature exceeds a critical point, the so-called Curie temperature, there is no spontaneous magnetization anymore and the material becomes paramagnetic. Despite the parallel alignment of the magnetic moments below the Curie temperature, ferromagnets may be macroscopically demagnetized in their initial state if no magnetic 


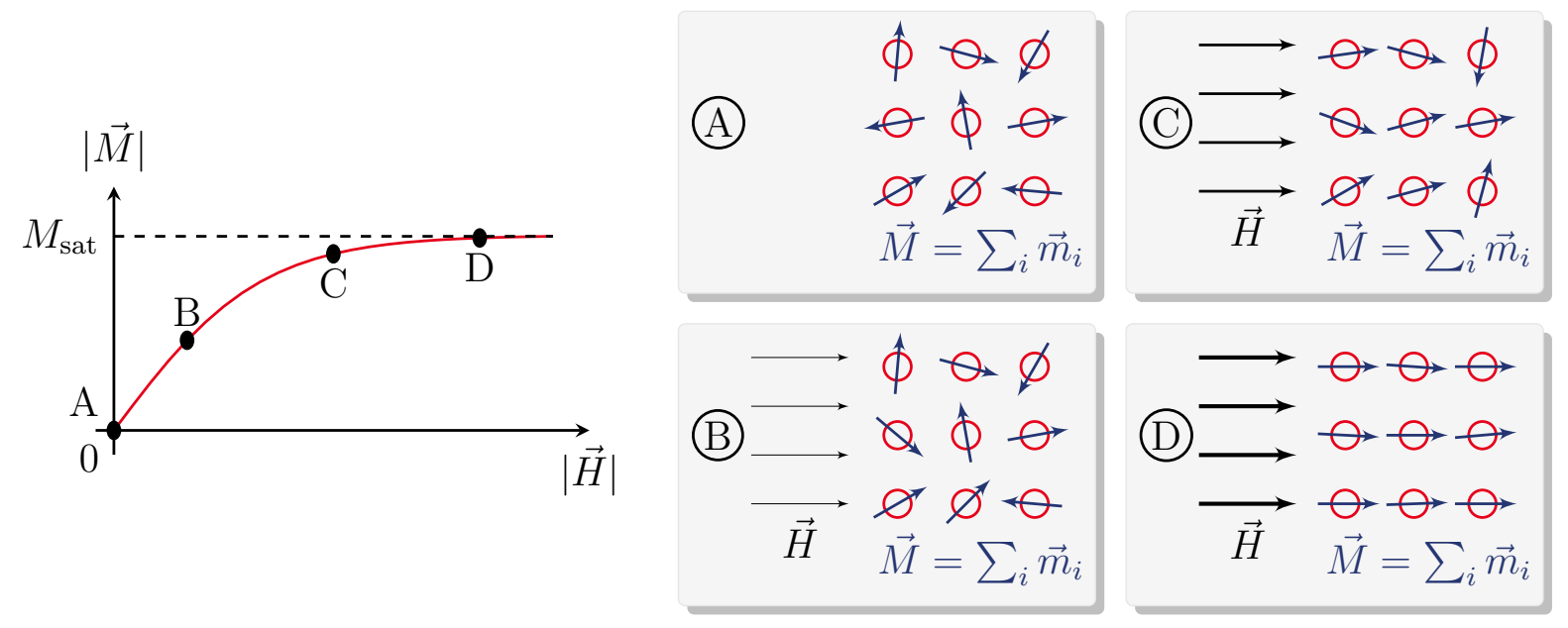

Figure 2.1.: Magnetization as a function of the applied magnetic field strength for a paramagnetic material. The pictures on the right illustrate the orientation of the microscopic magnetic moments $\vec{m}$ at the specified points. With increasing field strength, they gradually line up with the applied field $\vec{H}$ until the macroscopic magnetization $|\vec{M}|=\left|\sum_{i} \vec{m}_{i}\right|$ reaches its saturation value $M_{\text {sat }}$.

field is applied. The reason for this is that the material is divided into many magnetic domains (Weiss domains), in which the magnetic moments are parallel to each other. The direction of alignment of all domains is more or less random albeit the easy axes of the crystal lattice of magnetic anisotropic materials may be preferred. Moreover, the domains are separated by domain walls, where the orientation of the magnetic moments rotates gradually from the direction in one domain to the one of the other. Eventually, the fundamental reason for the formation of the domains and walls is the minimization of the internal energy [7]. When a magnetic field is applied, the size of the domains with an orientation in the direction of the external field increases at the expense of the others. This process is reversible for a small strength of the applied field but becomes irreversible for higher field strengths mainly due to displacements of domain walls over crystallographic inhomogeneities and defects. A further increased external field leads to rotation of the domains away from the easy axis, which is a predominantly reversible process. Finally, the magnetic moments of all domains are aligned with the external field and magnetic saturation is reached. Due to the involved irreversible processes, the (macroscopic) magnetization does not follow the same curve when the applied magnetic field strength is reduced again but shows hysteresis effects. In particular, even if the external field is absent there is still a net magnetization, called the remanence magnetization. The intensity of the magnetic field that is required to reduce the magnetization to zero again is denoted by coercivity. The magnetization process and the important magnetic quantities are also illustrated in figure 2.2.

\subsubsection{Antiferromagnetism and ferrimagnetism}

Antiferromagnetic and ferrimagnetic materials are characterized by two crystallographic sublattices whose magnetic moments are aligned in opposite direction to each other. In the case of antiferromagnetism, the contributions to the total magnetization of the two subsystems are identical in magnitude and thus cancel each other resulting in a vanishing macroscopic magneti- 

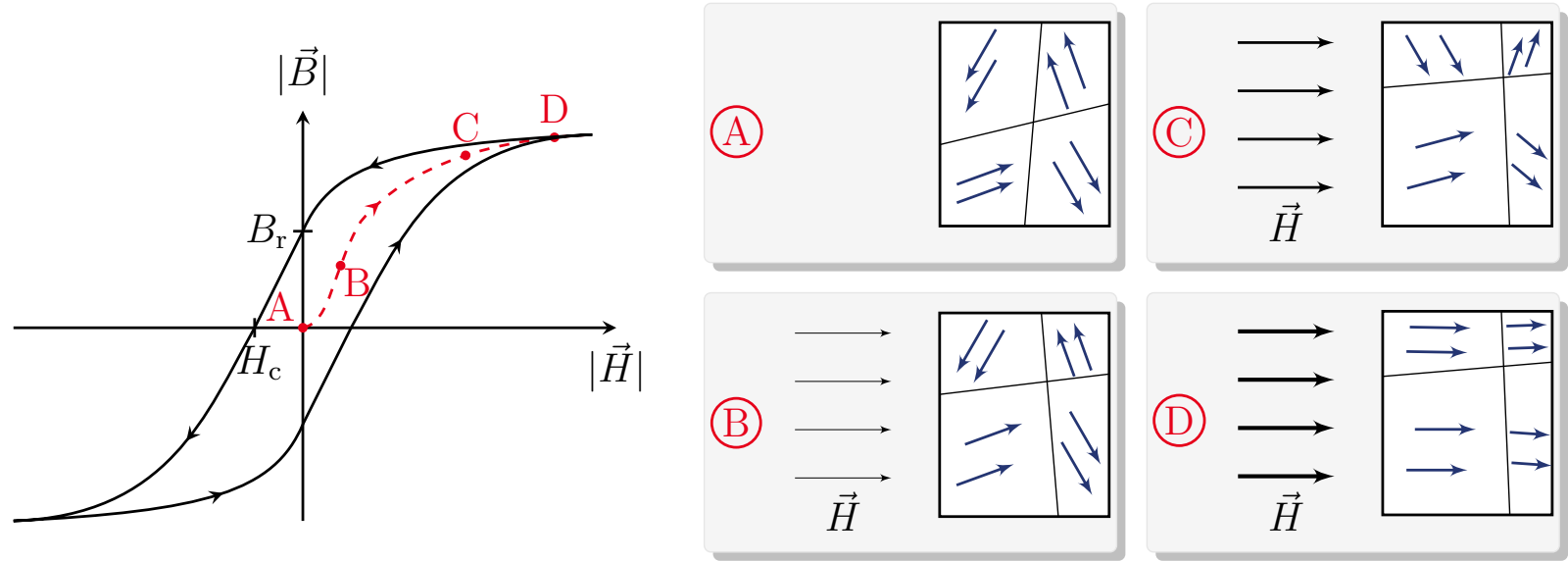

Figure 2.2.: Typical initial magnetization curve (dashed, red) and hysteresis loop (black, solid) of a ferromagnet. The pictures on the right illustrate the domain configuration for the selected points. The domains are distributed randomly in the demagnetized state (A). With increasing magnetic field strength, at first the size of the domains with an orientation in the direction of the external field increases (B), then domains are rotated $(C)$ until they are all lined up with the applied field in the magnetic saturated state (D). Moreover, the remanence (with residual induction $B_{\mathrm{r}}$ ) and the coercivity $H_{\mathrm{c}}$ are shown.

zation. In contrast, different magnetic ions occupy the sites of the two lattices for ferrimagnetic materials. Hence, a spontaneous magnetization of the substance remains. The magnetic properties are consequently similar to those of ferromagnetic material. Examples for ferrimagnetic substances are magnetic garnets, such as yttrium iron garnets, and ferrites. The latter are ceramic oxides with a chemical structure like magnetite $\left(\mathrm{Fe}_{3} \mathrm{O}_{4}\right)$ but with the divalent ferrous ion substituted with one or several different divalent transition metals [6]. Depending on the type of the compound metal, they are categorized into hard and soft ferrites. Whereas the former exhibit a high coercivity and high remanence after magnetization, the latter have a comparatively low coercivity. Among the most common soft ferrites are manganese-zinc ferrites (MnZn, with chemical composition $\mathrm{Mn}_{a} \mathrm{Zn}_{(1-a)} \mathrm{Fe}_{2} \mathrm{O}_{4}$ ) and the nickel-zinc ferrites (NiZn, with chemical composition $\mathrm{Ni}_{a} \mathrm{Zn}_{(1-a)} \mathrm{Fe}_{2} \mathrm{O}_{4}$ ). The latter feature a particularly high electrical resistivity, which prevents eddy currents in the cores. Also the Ferroxcube $8 \mathrm{C} 12 \mathrm{~m}$ material, which is installed inside the GSI SIS 18 cavities, is a NiZn ferrite.

\subsubsection{Magnetodynamics equation}

The dynamics of the magnetization subject to an external magnetic field is governed by the relation [5]

$$
\frac{\mathrm{d} \vec{M}}{\mathrm{~d} t}=\gamma_{e} \mu_{0}(\vec{M} \times \vec{H})
$$

This equation describes the precession movement of the magnetization around the axis defined by the external magnetic field $\vec{H}$ of strength $H$ (cf. Fig. 2.3(a)) with angular frequency

$$
\omega_{0}=-\gamma_{e} \mu_{0} H
$$


(a)

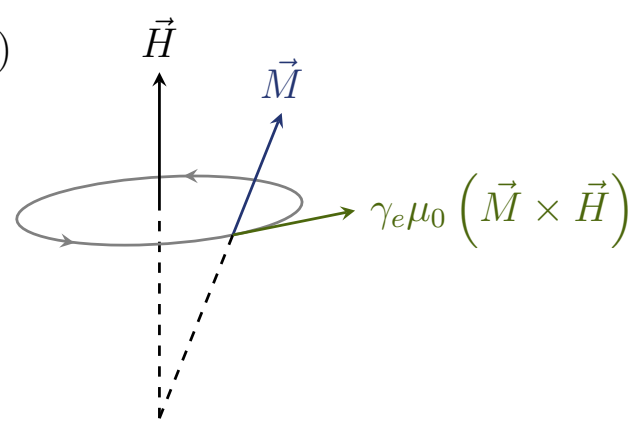

(b)

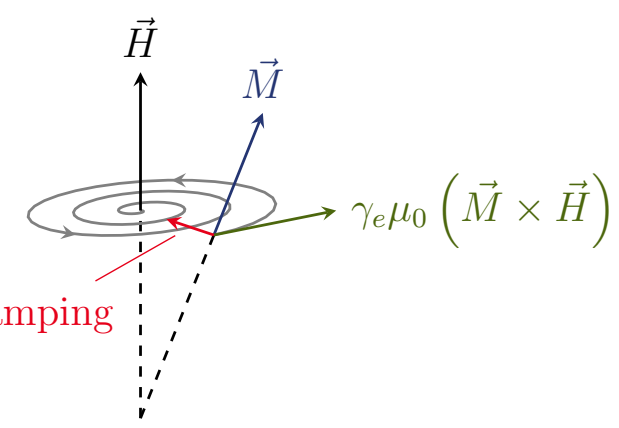

Figure 2.3.: Precession movement of the magnetization $\vec{M}$ around the external magnetic field $\vec{H}$ without (a) and including damping (b). The green arrows point towards the direction of the change of magnetization as described by equation (2.9), whereas the red one in (b) indicates the damping due to a term as in equation (2.13) or equation (2.14).

which is also known as Larmor frequency [5]. Therein, the gyromagnetic ratio is given by

$$
\gamma_{e}=-\frac{g e}{2 m_{e}}
$$

with $g$ being the Landé $g$-factor, $e$ the positive elementary charge and $m_{e}$ the electron mass. In order to take into account losses, damping terms have to be added to the right hand side of equation (2.9). Though several phenomenological terms were proposed in the past, the most common ones can be expressed in the general form of the ferromagnetic dynamical equation

$$
\frac{\mathrm{d} \vec{M}}{\mathrm{~d} t}=\gamma(\vec{M} \times \vec{H})-\frac{\alpha}{M^{2}}(\vec{M} \times(\vec{M} \times \vec{H}))-\beta \vec{M}
$$

which was obtained by Callen from quantum mechanical considerations [8]. Therein, $\gamma, \lambda$ and $\alpha$ are unknown scalar functions of $M, H$ and intrinsic characteristics of the material. By setting $\gamma=$ const. $=\gamma_{e} \mu_{0}=: \gamma_{\mathrm{LL}}, \alpha=$ const. $=: \lambda_{\mathrm{LL}}$ and $\beta=0$, the Landau-Lifshitz equation [9]

$$
\frac{\mathrm{d} \vec{M}}{\mathrm{~d} t}=\gamma_{\mathrm{LL}}(\vec{M} \times \vec{H})-\frac{\lambda_{\mathrm{LL}}}{M^{2}}(\vec{M} \times(\vec{M} \times \vec{H})),
$$

is obtained. The Landau-Lifshitz damping term results in a precession of the magnetization on gradually smaller cones until it is eventually aligned with the magnetic field (cf. Fig. 2.3(b)). Equation (2.13) can be expressed in the mathematically equivalent form

$$
\frac{\mathrm{d} \vec{M}}{\mathrm{~d} t}=\gamma_{\mathrm{G}}(\vec{M} \times \vec{H})+\frac{\alpha_{\mathrm{G}}}{M}\left(\vec{M} \times \frac{\mathrm{d} \vec{M}}{\mathrm{~d} t}\right)
$$

by defining $\gamma_{\mathrm{G}}=\gamma_{\mathrm{LL}}\left(1+\alpha_{\mathrm{G}}^{2}\right)$ and $\alpha_{\mathrm{G}}=-\lambda_{\mathrm{LL}} /\left(\gamma_{\mathrm{LL}} M\right)[10,11]$. This form, originally formulated this way (with $\gamma_{\mathrm{G}}=\gamma_{e} \mu_{0}$ ) by Gilbert [11], manifestly shows a damping proportional to the magnitude in the rate of change of $M$. Hence, despite the mathematical equivalence, the physical interpretation of the two distinct damping terms is quite different [12]; only in the limit of a small damping constant $\alpha_{\mathrm{G}} \ll 1$ the two coincide also from the physical point of view [6, 12]. 
This condition is met for single crystal ferrites, where $0.01<\alpha_{\mathrm{G}}<0.1$ [6]. Another class of damping terms are adapted from the Bloch equations [13], which were originally derived for the description of paramagnetic resonance. One common characteristic consists of a non-vanishing coefficient $\beta$ in the general equation (2.12). For instance, the so-called Bloch-Bloembergen equation takes the form [14]

$$
\frac{\mathrm{d} \vec{M}}{\mathrm{~d} t}=\gamma_{e}(\vec{M} \times \vec{H})-\vec{e}_{x} \frac{M_{x}}{T_{2}}-\vec{e}_{y} \frac{M_{y}}{T_{2}}-\vec{e}_{z} \frac{M_{z}-M_{0}}{T_{1}},
$$

where $T_{1}$ and $T_{2}$ are relaxation times of the longitudinal and transversal magnetization, respectively, $M_{x}, M_{y}$ and $M_{z}$ the $x$-, $y$ - and z-component of the magnetization, respectively, and $M_{0}$ the (static) saturation magnetization. Note that, unlike the Landau-Lifshitz as well as the Gilbert damping terms, the one in equation (2.15) does not conserve the magnitude of macroscopic magnetization and is therefore applicable to the description of relaxation processes in material with imperfect ferromagnetic order [15].

Throughout this work a Gilbert damping term as in equation (2.14) with $\gamma_{\mathrm{G}}=\gamma_{e} \mu_{0}$ will be used. Moreover, the explicit subscript of the damping constant will be omitted, i.e. $\alpha:=\alpha_{\mathrm{G}}$. Note that $\alpha$ is related to the line width $\Delta H_{0}$ as [6]

$$
\alpha=\frac{\gamma_{e} \Delta H_{0}}{2 \omega} .
$$

Therein, $\Delta H_{0}$ is defined as the difference between the two values of the magnetic field strength at which the imaginary part of the permeability takes half of its maximum value at resonance evaluated at a fixed frequency $\omega$.

\subsubsection{Permeability tensor}

The magnetodynamic equation discussed in the previous section is the basis for the derivation of the permeability tensor. It is thus clear that the tensor components depend on the magnetic properties of the material as well as on the strength and direction of the applied bias magnetic field. To begin with, an expression for the tensor will be derived for the fully magnetized state for a static magnetic field along the z-axis of the coordinate system analogously to [6]. Subsequently, the obtained formulas will be generalized for different magnetization states, namely for the fully demagnetized state in subsection 2.2.3.2 and the partially magnetized state in subsection 2.2.3.3. Finally, expressions for the tensor for a general orientation of the bias magnetic field are given in subsection 2.2.3.4.

\subsubsection{Fully saturated state}

For the derivation of the permeability tensor $\overleftrightarrow{\mu}$, the magnetic field and the magnetization in the relation (2.6) are explicitly decomposed into contributions constant in time and time-dependent parts, i.e.

$$
\begin{aligned}
\vec{H}(t) & =\vec{H}_{0}+\vec{H}_{d}(t)=\vec{H}_{0}+\operatorname{Re}\left(\vec{H}_{d} \cdot e^{\mathrm{i} \omega t}\right), \\
\vec{M}(t)=\vec{M}_{0}+\vec{M}_{d}(t) & =\vec{M}_{0}+\operatorname{Re}\left(\vec{M}_{d} \cdot e^{\mathrm{i} \omega t}\right) .
\end{aligned}
$$


In the absence of RF-components, that is $\left|\vec{H}_{d}\right|=\left|\vec{M}_{d}\right|=0$, the magnetization lines up with the static magnetic field, i.e. $\vec{H}_{0} \| \vec{M}_{0}$, at thermal equilibrium due to relaxation. For the following derivation, the coordinate system is set such that this orientation is aligned with the z-axis, i.e.

$$
\vec{H}_{0}=H_{0} \cdot \vec{e}_{z} ; \vec{M}_{0}=M_{0} \cdot \vec{e}_{z}
$$

Throughout this subsection, only the fully magnetized state is considered, where the bias field strength is assumed to be large compared to the superimposed RF, that is to say, $\left|\vec{H}_{d}\right| \ll\left|\vec{H}_{0}\right|$ and consequently $\left|\vec{M}_{d}\right| \ll\left|\vec{M}_{0}\right|$ with $M_{0}=M_{\text {sat }}$. Furthermore, hysteresis effects are not taken into account. Substituting equations (2.17) and (2.18) into the equation of motion for the magnetization including the Gilbert damping term (2.14) then yields

$$
i \omega \vec{M}_{d}=\gamma_{e} \mu_{0} M_{\mathrm{sat}}\left(\vec{e}_{z} \times \vec{H}_{d}\right)+\left(-\gamma_{e} \mu_{0} H_{0}+\mathrm{i} \omega \alpha\right)\left(\vec{e}_{z} \times \vec{M}_{d}\right)
$$

where terms of order two in the components of the time-harmonic contributions $M_{d}$ and $H_{d}$ are neglected according to the above assumption. Evaluating the cross-products in equation (2.19) and simultaneously solving for the three vector components of $\vec{M}_{d}$ in terms of $\vec{H}_{d}$, one obtains the susceptibility tensor from the relation

$$
\vec{M}_{d}=\overleftrightarrow{\chi}_{m} \vec{H}_{d}
$$

and from this finally the permeability tensor

$$
\overleftrightarrow{\mu}=\mu_{0}\left(\mathbb{1}_{3 \times 3}+\overleftrightarrow{\chi_{m}}\right)
$$

as $^{1}$

$$
\overleftrightarrow{\mu}=\mu_{0}\left(\begin{array}{ccc}
\mu_{\text {diag }} & -\mathrm{i} \kappa & 0 \\
\mathrm{i} \kappa & \mu_{\text {diag }} & 0 \\
0 & 0 & \mu_{z}
\end{array}\right) \quad\left(\text { for } \vec{H}_{0} \| \vec{e}_{z}\right)
$$

with

$$
\begin{aligned}
\mu_{\text {diag }} & =1+\chi \\
\chi & =\frac{\left(\omega_{0}+i \omega \alpha\right) \omega_{\mathrm{M}}}{\left(\omega_{0}+i \omega \alpha\right)^{2}-\omega^{2}}, \\
\kappa & =\frac{-\omega \omega_{\mathrm{M}}}{\left(\omega_{0}+\mathrm{i} \omega \alpha\right)^{2}-\omega^{2}}, \\
\omega_{0} & =-\gamma_{e} \mu_{0} H_{0}, \\
\omega_{\mathrm{M}} & =-\gamma_{e} \mu_{0} M_{\mathrm{sat}}, \\
\text { and } \mu_{z} & =1 .
\end{aligned}
$$

$1 \quad$ Note that the position of the minus sign in the off-diagonal elements depends on the sign chosen in the exponent of the time dependent part of the fields (cf. Eq. (2.17)). Hence, the occurrence of the minus sign is found to be interchanged in some textbooks. 
In this form the tensor was first formulated by Polder [16] and is therefore often referred to as Polder tensor. If one of the alternative damping terms introduced in subsection 2.2.2 is considered instead of the Gilbert term, the tensor structure (2.22) remains unchanged. Only the expressions for its components (2.23) have to be replaced by corresponding ones.

As mentioned in subsection 1.1.1, the bias current and thus the resulting magnetization of the ferrite material is modified in a rather wide range during one operation cycle of a ferrite-loaded cavity. Consequently, for an accurate computation of the eigenfrequencies also for low bias field strengths, modified expressions for the partially magnetized state are required. In this regime ferrites can by described by an effective permeability tensor, which depends on local properties [17] and which is discussed in the following subsections beginning with the fully demagnetized state.

\subsubsection{Fully demagnetized state}

In the completely demagnetized state, the effective permeability tensor reduces to a scalar since the medium is assumed to be effectively isotropic due to the randomly oriented domains. Its form was first derived by Schlömann [18]. Assuming only spin up and down domains (aligned along the $z$-direction), whose local permeability is described by a tensor of the form of equation (2.22) with $H_{0}$ resulting from the anisotropy field, the effective permeability tensor in the case of a cylindrically symmetric domain configuration takes the form

$$
\overleftrightarrow{\mu}=\mu_{0}\left(\begin{array}{ccc}
\mu_{\mathrm{eff}} & 0 & 0 \\
0 & \mu_{\mathrm{eff}} & 0 \\
0 & 0 & \mu_{z}
\end{array}\right)
$$

where

$$
\mu_{\mathrm{eff}}=\sqrt{\mu_{\mathrm{diag}}^{2}-\kappa^{2}}
$$

with $\mu_{\text {diag }}$ and $\kappa$ as defined in equations (2.23). This value may be interpreted as the geometric average $\mu_{\text {eff }}=\sqrt{\mu_{+} \mu_{-}}$of the permeabilities $\mu_{+}=\mu_{\text {diag }}-\kappa$ and $\mu_{-}=\mu_{\text {diag }}+\kappa$ for clockwise and counterclockwise rotating circularly polarized fields, respectively [19]. Averaging over all directions for random orientation of up and down domains, yields the scalar isotropic permeability

$$
\hat{\mu}=\frac{1}{3}\left(\mu_{z}+2 \sqrt{\mu_{\text {diag }}^{2}-\kappa^{2}}\right)
$$

with $\mu_{z}=1$ as in equation (2.23f). Despite of the rather restrictive assumptions, it reproduces experimental results rather well [20]. Moreover, an alternative derivation using effectivemedium approximation by Bouchaud and Zérah [21] confirms the above formula also from the theoretical point of view.

\subsubsection{Partially magnetized state}

The partially magnetized state is characterized by the reduced magnetization, defined as the ratio of the magnitude of the present magnetization to the saturation magnetization $m=M / M_{\text {sat }}$, 
being in the range $0<m<1$. The first formulation of the tensor in this regime was proposed by Rado [22] for the limit $\omega_{0} \ll \omega$ and $\omega_{M} \ll \omega$. Without taking into account magnetic losses and provided that the average magnetization points into z-direction, starting from the expression for the fully magnetized state (2.22) and (2.23) these conditions result in a tensor of the form of equation (2.22) with 1 as the main diagonal elements, i.e. $\mu_{\text {diag }}=\mu_{z}=1$, and the off-diagonal term given by

$$
\widetilde{\kappa}=-m \frac{\omega_{\mathrm{M}}}{\omega} .
$$

It is found that equation (2.27) provides a reasonably good approximation for the real part of the off-diagonal element as a function of frequency for particular materials [20, 23]. However, it suffers from the inaccuracy of the main diagonal terms. Based on measurements on yttrium iron garnets and magnesium-manganese (zinc) ferrites, Green and Sandy formulated empirical expressions for $\overleftrightarrow{\mu}[17,23]$. According to this, the real part of the main diagonal elements can be described well by

$$
\begin{aligned}
\tilde{\mu}_{\text {diag }} & =\hat{\mu}+(1-\hat{\mu}) m^{1.5}, \\
\tilde{\mu}_{z} & =\hat{\mu}^{\left(1-m^{2.5}\right)} \\
\text { with } \hat{\mu} & =\frac{1}{3}\left(1+2 \sqrt{1-\left(\frac{\omega_{\mathrm{M}}}{\omega}\right)^{2}}\right) .
\end{aligned}
$$

Note that equation (2.28c) coincides with the effective scalar permeability for the completely demagnetized state (cf. Eq. (2.26)) for vanishing anisotropy magnetic fields and losses, i.e. $H_{0}=0$ and $\alpha=0$. For incorporation of losses, the above authors further assume the imaginary part of the off-diagonal elements to be negligible, whereas $\operatorname{Im} \widetilde{\mu}_{\text {diag }}=\operatorname{Im} \widetilde{\mu}_{z}=$ const. throughout the partially magnetized state. The main flaw of the above formulation is that it does not agree with the Polder tensor (2.22) in the limit of the fully magnetized state. But even more important for the study of ferrite loaded-cavities, the formulas are not necessarily applicable below gyromagnetic resonance, where $\omega<\omega_{0}$, due to the made assumptions.

An alternative expression for the effective permeability tensor is presented by Igarashi and Naito [24]. They solved the equation of motion for the magnetization (2.14) for a static magnetic field with general orientation

$$
\alpha_{x} \vec{e}_{x}+\alpha_{y} \vec{e}_{y}+\alpha_{z} \vec{e}_{z} \text { with } \alpha_{x}^{2}+\alpha_{y}^{2}+\alpha_{z}^{2}=1
$$

Analogous to the derivation of Rado's formula, spatial averaging, denoted by $<\cdots>$, with random domain orientations and the approximation

$$
1-\left\langle\alpha_{z}^{2}\right\rangle \simeq 1-\left\langle\alpha_{z}\right\rangle^{2}=1-m^{2}
$$

finally lead to

$$
\overleftrightarrow{\mu}=\mu_{0}\left(\begin{array}{ccc}
1+\frac{1}{2} \chi\left(1+m^{2}\right) & -\mathrm{i} m \kappa & 0 \\
\mathrm{i} m \kappa & 1+\frac{1}{2} \chi\left(1+m^{2}\right) & 0 \\
0 & 0 & 1+\chi\left(1-m^{2}\right)
\end{array}\right) \quad\left(\text { for } \vec{H}_{0} \| \vec{e}_{z}\right)
$$


Therein $\chi$ and $\kappa$ are defined as for the fully magnetized state (cf. Eq. (2.23)), except that $H_{0}$ is considered as a free parameter, which has to be determined from experiment. This tensor agrees both with the one of Rado's theory in the validity limit (i.e. $\omega_{0} \ll \omega$ and $\omega_{M} \ll \omega$ ) and with the Polder tensor for the fully magnetized state. However, Igarashi and Naito claim that the main diagonal elements are not in accordance with experimental data for partially magnetized ferrites.

All the before mentioned approaches do not take into account a coupling of the magnetic moments of adjacent domains. This is accomplished by the self-consistent model of Gelin and Berthou-Pichavant [25]. Therein, the ferrite is regarded as an assembly of independent grains with random orientation. Each of the grains consists of interacting domains with magnetization parallel or anti-parallel to its easy axis. For the calculation of the permeability tensor components, at first the modified internal magnetic field and the magnetization equilibrium direction due to the applied external static magnetic field are determined in each domain. In order to incorporate interactions of adjacent domains via the Polder-Smit effect [26], two coupled equations of motion with Gilbert damping term (cf. Eq. (2.14)) are solved in the second step. Eventually, the global effective tensor components are obtained by spatial averaging of the individual contributions over all grains. To sum up, given the saturation magnetization, the magnetocrystalline anisotropy field, the demagnetizing coefficient depending on the shape of the domains as well as the (Gilbert) loss factor $\alpha$, this model provides analytical integral expressions for the components of the permeability tensor with arbitrary magnetization state. Though accordance of this theory with previous approaches in their validity limits is confirmed [25], Gelin and Quéffélec point to the incorrect description of the loss mechanism for low field bias and unsatisfactory results for the internal static magnetic field vector [20]. Hence, these two authors generalized the Gelin-Berthou model [20] by introducing statistical distribution laws for domain and grain demagnetizing coefficients as well as by incorporating the Stoner-Wohlfarth hysteresis model [27]. The latter enables the reproduction of the second order hysteresis effect [20], which was observed in experiments by Green and Sandy [23].

In the above expressions for the permeability tensor, the static magnetic field is assumed to be parallel to the $z$-axis of the coordinate system. Yet, a numerical solution of the curl-curl equation (3.5) of problems with a non-homogeneous bias field requires a form of the tensor for a fully general orientation of $\vec{H}_{0}$, which is derived in the following subsection.

\subsubsection{Permeability tensor for arbitrary orientation of the bias magnetic field}

With the aim of deriving an expression for the permeability tensor when the bias magnetic field is not aligned with the $z$-axis, let the general direction of the bias magnetic field be defined by the unit vector

$$
\begin{array}{r}
\vec{e}_{r}:=\left(\begin{array}{c}
\sin \theta \cos \phi \\
\sin \theta \sin \phi \\
\cos \theta
\end{array}\right), \\
\text { i.e. } \vec{H}_{0}=H_{0} \cdot \vec{e}_{r} ; \vec{M}_{0}=M_{0} \cdot \vec{e}_{r} \text {. }
\end{array}
$$

Herein, $\theta$ and $\phi$ are the polar and azimuthal angle in the spherical coordinate system, respec- 


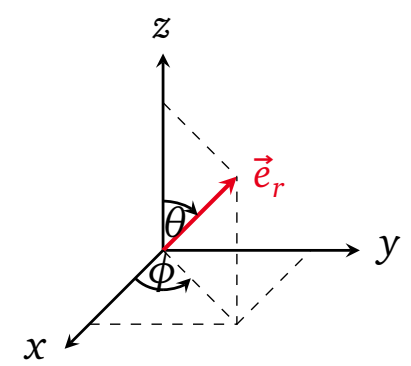

Figure 2.4.: Unit vector $\vec{e}_{r}$ in the spherical coordinate system with the polar angle $\theta$ and the azimuthal angle $\phi$.

tively (see also Fig. 2.4). Repeating the same procedure as explained in subsection 2.2.3.1 with vectors (2.32) instead of (2.18) leads to the permeability tensor

$$
\overleftrightarrow{\mu}(\theta, \phi)=\mu_{0}\left(\begin{array}{lll}
\mu_{x, x}(\theta, \phi) & \mu_{x, y}(\theta, \phi) & \mu_{x, z}(\theta, \phi) \\
\mu_{y, x}(\theta, \phi) & \mu_{y, y}(\theta, \phi) & \mu_{y, z}(\theta, \phi) \\
\mu_{z, x}(\theta, \phi) & \mu_{z, y}(\theta, \phi) & \mu_{z, z}(\theta, \phi)
\end{array}\right)
$$

with

$$
\begin{aligned}
& \mu_{x, x}(\theta, \phi)=\cos ^{2} \phi\left(\mu_{\text {diag }} \cos ^{2} \theta+\mu_{z} \sin ^{2} \theta\right)+\mu_{\text {diag }} \sin ^{2} \phi, \\
& \mu_{x, y}(\theta, \phi)=\cos \phi\left(-\mu_{\text {diag }}+\mu_{z}\right) \sin ^{2} \theta \sin \phi-\mathrm{i} \kappa \cos \theta, \\
& \mu_{x, z}(\theta, \phi)=\sin \theta\left(\cos \theta \cos \phi\left(-\mu_{\text {diag }}+\mu_{z}\right)+\mathrm{i} \kappa \sin \phi\right), \\
& \mu_{y, x}(\theta, \phi)=\cos \phi\left(-\mu_{\text {diag }}+\mu_{z}\right) \sin ^{2} \theta \sin \phi+\mathrm{i} \kappa \cos \theta, \\
& \mu_{y, y}(\theta, \phi)=\mu_{\text {diag }} \cos \phi^{2}+\left(\mu_{\text {diag }} \cos ^{2} \theta+\mu_{z} \sin ^{2} \theta\right) \sin ^{2} \phi, \\
& \mu_{y, z}(\theta, \phi)=\sin \theta\left(\cos \theta\left(-\mu_{\text {diag }}+\mu_{z}\right) \sin \phi-i \kappa \cos \phi\right), \\
& \mu_{z, x}(\theta, \phi)=-\sin \theta\left(\cos \theta \cos \phi\left(\mu_{\text {diag }}-\mu_{z}\right)+\mathrm{i} \kappa \sin \phi\right), \\
& \mu_{z, y}(\theta, \phi)=\sin \theta\left(\cos \theta\left(-\mu_{\text {diag }}+\mu_{z}\right) \sin \phi+\mathrm{i} \kappa \cos \phi\right), \\
& \mu_{z, z}(\theta, \phi)=\mu_{z} \cos ^{2} \theta+\mu_{\text {diag }} \sin ^{2} \theta .
\end{aligned}
$$

This expression is in accordance with the one given in [28] for $\mu_{\text {diag }}=1+\chi$ and $\mu_{z}=1$, which can easily be seen after applying some trigonometric identities (and taking into account the different sign convention for $\kappa$ therein). Obviously, after substituting the tensor components $\mu_{\text {diag }}, \kappa$ and $\mu_{z}$ for the effective tensor components, this expression is also applicable to the partially magnetized state. Alternatively, the general form of the tensor (2.33) can readily be obtained by applying the orthogonality transformation

$$
\overleftrightarrow{\mu}(\theta, \phi)=R(\theta, \phi)^{T} \overleftrightarrow{\mu} R(\theta, \phi)
$$

with the rotation matrix in three dimensions $R(\theta, \phi)$ to the original tensor for $\vec{H}_{0} \| \vec{e}_{z}$.

\subsubsection{Permittivity tensor}

Though ferrites exhibit only purely gyromagnetic properties, the problem of the calculation of eigensystems in chapter 3 will be formulated for materials with general gyrotropic characteristics to allow a broader field of application. Hence, also the behavior of gyroelectric substances 
is discussed briefly in this subsection using the example of a magnetized plasma. A plasma is an electrically quasi-neutral medium consisting of a negatively-charged electron gas, which moves freely in front of a background of positive ions. If the electron gas is displaced by the distance $|\vec{x}|$ with respect to the ions, the (electric) dipole moment

$$
\vec{P}=N_{e} e \vec{x}
$$

is induced, where $N_{e}$ is the number of electrons per unit volume and $e$ the (positive) elementary charge. Due to the Coulomb force, the electrons oscillate with respect to the ions while being damped by particle collisions, which is quantified by the collision frequency $\mathrm{v}_{\mathrm{c}}$. Moreover, in the presence of a static magnetic field $\vec{B}_{0}$ with a large field strength compared to $R F$-components, the Lorentz-force must not be neglected. Hence, the time-dependence of the displacement of the electrons in a magnetized plasma can be described approximately by the differential equation [29]

$$
m_{e} \frac{\mathrm{d}^{2} \vec{x}}{\mathrm{~d} t^{2}}+m_{e} \mathrm{v}_{\mathrm{c}} \frac{\mathrm{d} \vec{x}}{\mathrm{~d} t}=-e\left(\vec{E}+\frac{\mathrm{d} \vec{x}}{\mathrm{~d} t} \times \vec{B}_{0}\right)
$$

In the absence of a magnetic field, the electrons oscillate accordingly with the so-called plasma frequency

$$
\omega_{\mathrm{p}}=\sqrt{\frac{N_{e} e^{2}}{\varepsilon_{0} m_{e}}}
$$

whereas the cyclotron frequency of the charged particles in the magnetic field is given by

$$
\omega_{\mathrm{b}}=\frac{B_{0} e}{m_{e}} .
$$

Inserting relation (2.36) into the differential equation (2.37) and employing the above definitions (2.38) and (2.39), leads to the (electric) susceptibility tensor

$$
\vec{P}=\varepsilon_{0} \overleftrightarrow{\chi_{e}} \vec{E}
$$

and from this to the permittivity tensor

$$
\overleftrightarrow{\varepsilon}=\varepsilon_{0}\left(\mathbb{1}_{3 \times 3}+\overleftrightarrow{\chi_{e}}\right)
$$

If the plasma is experiencing a magnetic field in $z$-direction, i.e. $\vec{B}_{0}=B_{0} \cdot \vec{e}_{z}$, the tensor takes the form [29]

$$
\overleftrightarrow{\varepsilon}=\varepsilon_{0}\left(\begin{array}{ccc}
\varepsilon_{1} & -\mathrm{i} \varepsilon_{2} & 0 \\
\mathrm{i} \varepsilon_{2} & \varepsilon_{1} & 0 \\
0 & 0 & \varepsilon_{3}
\end{array}\right) \quad\left(\text { for } \vec{B}_{0} \| \vec{e}_{z}\right)
$$


with

$$
\begin{aligned}
& \varepsilon_{1}=1-\frac{\omega_{\mathrm{p}}^{2}\left(\omega-\mathrm{iv}_{\mathrm{c}}\right)}{\omega\left(\left(\omega-\mathrm{iv}_{\mathrm{c}}\right)^{2}-\omega_{\mathrm{b}}^{2}\right)}, \\
& \varepsilon_{2}=\frac{\omega_{\mathrm{p}}^{2} \omega_{\mathrm{b}}}{\omega\left(\left(\omega-\mathrm{iv}_{\mathrm{c}}\right)^{2}-\omega_{\mathrm{b}}^{2}\right)}, \\
& \varepsilon_{3}=1-\frac{\omega_{\mathrm{p}}^{2}}{\omega\left(\omega-\mathrm{i} \mathrm{v}_{\mathrm{c}}\right)}
\end{aligned}
$$

Furthermore, the permittivity tensor for a general orientation of the magnetic field can readily be obtained out of the above expression by applying the orthogonality transformation (cf. subsection 2.2.3.4)

$$
\overleftrightarrow{\varepsilon}(\theta, \phi)=R(\theta, \phi)^{T} \overleftrightarrow{\varepsilon} R(\theta, \phi)
$$

\subsection{Permeability measurement}

In the theoretical description of the properties of gyrotropic material of the previous section, a couple of a-priori unknown parameters remain for a quantitative description. These parameters have to be determined from experimental data for each particular material. As mentioned above, it is the objective of the presented thesis to develop a tool for the numerical simulation of the lowest eigenmodes of ferrite-loaded cavities. Evidently, accurate material characteristics are inevitable ingredients for the numerical computations. Since ferrites exhibit only purely gyromagnetic and no gyroelectric properties, only the former are considered in the following. Due to the limited information available in the data sheet provided by the manufacturer, own experiments were carried out at the GSI facility. The aim of these measurements is to gain knowledge about the magnetic properties of the Ferroxcube $8 \mathrm{C} 12 \mathrm{~m}$ ferrite material in the parameter range where the GSI SIS 18 cavity resonators are operated. Among the properties of interest are the $B-H$ curve, the frequency characteristic of both the real and imaginary part of the permeability as well as its dependence on the presence of a (quasi static) bias magnetic field of variable strength. The measurement of these magnetic characteristics and its analysis are discussed in the following section. Firstly, the determination of the complex permeability in a reflection and a transmission setup is discussed including an extensive uncertainty analysis. Finally, results of the measurement of the $B-H$ curve which is enclosed throughout a reduced working cycle of the cavity are presented.

This section is basically a reproduction of the part about the material measurement of the publication [30], which is supplemented with a quantitative uncertainty analysis.

\subsubsection{Measurement setup}

Two different methods are used for the measurement of the frequency-dependent permeability. Whereas the first approach is based on the measurement of the input port reflection coefficient, the $S_{11}$-parameter, transmission measurements of $S$-parameters are carried out in the second 

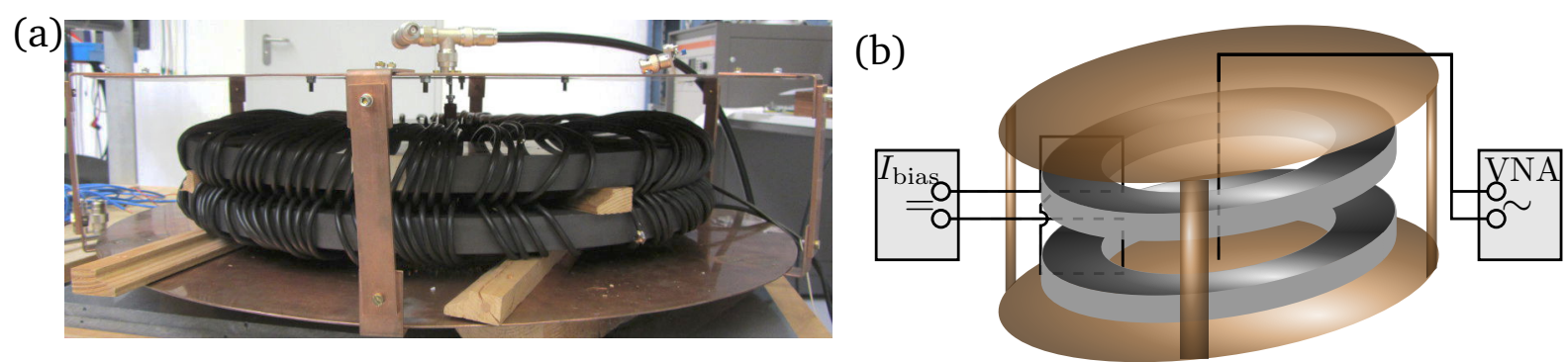

Figure 2.5.: Picture (a) and schematic view (b) of the experimental setup in the reflection measurement mode. The ferrite ring pair with bias windings is located inside the cavity housing. The $\mathrm{N}$-type cable in the center on top is connected to the VNA. A $50 \Omega$ resistor and optionally external capacitors can be installed in parallel.

Table 2.1.: Settings of the network analyzer for both measurement methods.

\begin{tabular}{r|l}
\hline \hline Number of sample points & 4001 \\
Sweep type & logarithmic frequency \\
Source power & $0 \mathrm{dBm}$ \\
Frequency range & $0.5 \mathrm{MHz}$ to $20 \mathrm{MHz}$ \\
Bandwidth & $1 \mathrm{kHz}$ \\
Averaging & 4 times \\
\hline \hline
\end{tabular}

one. The basic setting of both approaches is identical: Two Ferroxcube $8 \mathrm{C} 12 \mathrm{~m}$ full size toroids can be biased via $N_{\text {bias }}=105$ crossed (figure-of-eight) current windings. The bias current is adjusted manually at the direct current (DC) power supply (Delta Elektronika SM 45-70D [31]). Moreover, the rings together with the current windings are installed inside a copper cavity housing. For the first approach, this housing together with one centric wire, which connects the upper and lower plate, also serves for the coupling of the RF to the ring cores. In contrast, for the second approach the inner wire is disconnected. Instead, two separate current windings wound closely around both ring cores are installed for excitation and pick-up of the signal. The $S$-parameters of the device under test are measured with a Rohde \& Schwarz ZNB4 vector network analyzer [32] in the frequency range from $0.5 \mathrm{MHz}$ to $20 \mathrm{MHz}$. The used settings of the network analyzer (VNA) are listed in table 2.1. Optionally, external capacitors, which are soldered directly on a N-type-adapter to minimize the parasitic inductance of the connecting wires, can be installed in parallel. Figure 2.5(a) shows the measurement station for the reflection setup, figure 2.5 (b) its simplified schematic view.

In order to prepare the magnetic remanence state, the bias current is driven up to the maximum value of $50 \mathrm{~A}(\approx 4.6 \mathrm{kA} / \mathrm{m})$ for a short time in an initialization step. The measurement is carried out for values of the bias current starting with $0 \mathrm{~A}$ and raising up to the maximum value with enlarging increments. For the reflection measurement this is repeated with 1 and 2 capacitors with a capacitance of $1 \mathrm{nF}$ each installed in parallel. The temperature of the ring cores is controlled with a FLUKE 62 Mini IR thermometer [33]. Since no cooling system was installed, the temperature at the surface of the material increased from $23^{\circ} \mathrm{C}$ to about $32^{\circ} \mathrm{C}$ during the measurements due to the heating of the ferrite material. In order to reduce inaccuracies due to residual impedances, a careful calibration of the network analyzer including the N-type cable (type RG 214/U) was performed. Besides, a high phase stability was observed. 


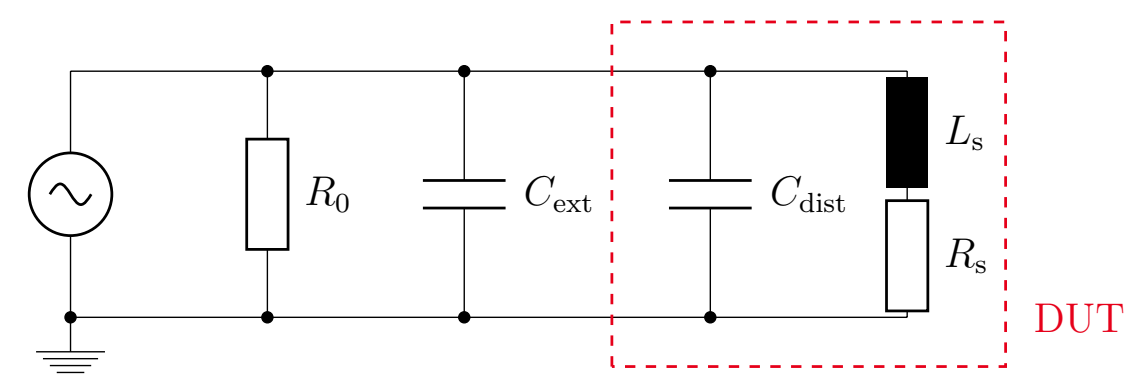

Figure 2.6.: Equivalent circuit diagram to represent the measurement setup. The resistor $R_{0}$ and optional external capacitors $C_{\text {ext }}$ are installed in parallel to the device under test (DUT). The DUT itself is modeled as a distributed capacitance $C_{\text {dist }}$ in parallel with a series inductance $L_{\mathrm{s}}$ and resistance $R_{\mathrm{s}}$.

The basic principle of the introduced methods are thus similar to approaches known from literature, namely for instance [34-37] for the reflection measurement and [38, 39] for the transmission measurement.

\subsubsection{Analysis of the reflection measurement data}

\subsubsection{General procedure}

The network analyzer records the absolute value and phase of the $S_{11}$-parameter at every sample point. Hence, after the reflection measurement the pair of values is available as a function of frequency for the different bias current and capacitor settings. This reflection parameter is transformed to the real and imaginary part of the admittance according to the relations [40]

$$
\begin{aligned}
& \operatorname{Re}(Y)=\frac{1-\left|S_{11}\right|^{2}}{1+\left|S_{11}\right|^{2}+2\left|S_{11}\right| \cos \left(\arg \left(S_{11}\right)\right)} \times \frac{1}{Z_{0}} \\
& \operatorname{Im}(Y)=\frac{-2\left|S_{11}\right| \sin \left(\arg \left(S_{11}\right)\right)}{1+\left|S_{11}\right|^{2}+2\left|S_{11}\right| \cos \left(\arg \left(S_{11}\right)\right)} \times \frac{1}{Z_{0}}
\end{aligned}
$$

where $Z_{0}=50 \Omega$ is the characteristic impedance. The question how the permeability can be extracted from the admittance data is discussed in the following. Assuming that the system can be described by a circuit as depicted in figure 2.6, its total admittance is given by

$$
Y=\frac{1}{R_{0}}+\mathrm{i} \omega C_{\mathrm{ext}}+\mathrm{i} \omega C_{\mathrm{dist}}+\frac{1}{\mathrm{i} \omega L_{\mathrm{s}}+R_{\mathrm{s}}}
$$

with the external and distributed capacitances $C_{\text {ext }}$ and $C_{\text {dist }}$, respectively, the parallel resistor $R_{0}=50 \Omega$ and the series inductance $L_{\mathrm{s}}$ and series resistance $R_{\mathrm{s}}$ of the two toroids. In reality, neither the parallel resistor nor the external capacitor behave as ideal components. To account for this, an additional measurement is carried out to obtain the admittance

$$
Y_{0}=\frac{1}{R_{0}}+\mathrm{i} \omega C_{\text {ext }}
$$




\begin{tabular}{c|c}
\hline \hline Quantity & Value \\
\hline$h_{\text {core }}$ & $50 \mathrm{~mm}$ \\
$r_{\text {out }}$ & $249 \mathrm{~mm}$ \\
$r_{\text {in }}$ & $136 \mathrm{~mm}$ \\
$r_{\text {cav,out }}$ & $340 \mathrm{~mm}$ \\
$r_{\text {cav,in }}$ & $1.75 \mathrm{~mm}$ \\
$h_{\text {cav }}$ & $143 \mathrm{~mm}$ \\
\hline \hline
\end{tabular}

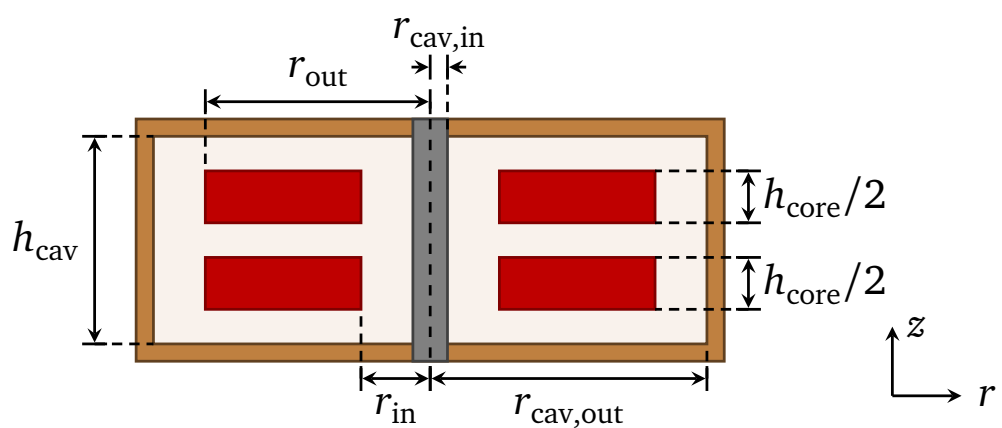

Figure 2.7.: Dimensions of the cavity and the ring cores. The illustration on the right shows a simplified two-dimensional sectional view through the center of the cavity. [30]

of these two components alone without being connected to the device under test (DUT). Hence, the inductance $L_{\mathrm{s}}$ and the resistance $R_{\mathrm{s}}$ of the ring cores can be expressed in terms of $Y$ and $Y_{0}$ as

$$
\begin{aligned}
L_{\mathrm{s}} & =\frac{\omega C_{\mathrm{dist}}-\operatorname{Im}(Y)+\operatorname{Im}\left(Y_{0}\right)}{\omega\left(\left(\operatorname{Re}(Y)-\operatorname{Re}\left(Y_{0}\right)\right)^{2}+\left(\operatorname{Im}(Y)-\operatorname{Im}\left(Y_{0}\right)-\omega C_{\mathrm{dist}}\right)^{2}\right)} \\
\text { and } \quad & R_{\mathrm{s}}=\frac{\operatorname{Re}(Y)-\operatorname{Re}\left(Y_{0}\right)}{\left(\operatorname{Re}(Y)-\operatorname{Re}\left(Y_{0}\right)\right)^{2}+\left(\operatorname{Im}(Y)-\operatorname{Im}\left(Y_{0}\right)-\omega C_{\mathrm{dist}}\right)^{2}},
\end{aligned}
$$

respectively. Moreover, these quantities are related to the permeability $\mu_{\mathrm{s}}=\mu_{\mathrm{s}}^{\prime}-\mathrm{i} \mu_{\mathrm{s}}^{\prime \prime}$ involving the dimensional parameters as depicted in figure 2.7 by the relations [36]

$$
\begin{aligned}
& L_{\mathrm{s}}=\frac{1}{\underline{I}} \int \underline{B} \mathrm{~d} A=\int_{r_{\text {cav }, \text { in }}} \int_{0}^{r_{\text {cav }, \text { out }}} \frac{\mu_{\mathrm{s}}}{2 \pi r} \mathrm{~d} r \mathrm{~d} z, \\
& R_{\mathrm{s}}=\frac{\mathrm{i} \omega}{\underline{I}} \int \underline{B} \mathrm{~d} A=\omega \int_{r_{\text {cav }, \text { in }}^{\prime}}^{r_{\text {cav, out }}} \int_{0}^{h_{\text {cav }}} \frac{\mu_{\mathrm{s}}^{\prime \prime}}{2 \pi r} \mathrm{~d} r \mathrm{~d} z .
\end{aligned}
$$

This can be transformed into

$$
\begin{aligned}
& L_{\mathrm{s}}=\frac{1}{2 \pi} h_{\text {core }}\left(\mu_{\mathrm{s}}^{\prime}-\mu_{0}\right) \ln \frac{r_{\mathrm{out}}}{r_{\mathrm{in}}}+L_{\mathrm{air}}, \\
& R_{\mathrm{s}}=\frac{\omega}{2 \pi} h_{\mathrm{core}} \mu_{\mathrm{s}}^{\prime \prime} \ln \frac{r_{\mathrm{out}}}{r_{\mathrm{in}}}+R_{\mathrm{cav}}
\end{aligned}
$$

with the inductance of the air-filled resonator

$$
L_{\text {air }}=\frac{1}{2 \pi} h_{\text {cav }} \mu_{0} \ln \frac{r_{\text {cav,out }}}{r_{\text {cav,in }}}
$$

and the resistance of the empty cavity $R_{\text {cav }}$. From equations (2.50), the real and imaginary part of the permeability are finally obtained as

$$
\begin{aligned}
& \mu_{\mathrm{s}}^{\prime}=\left(L_{\mathrm{s}}-L_{\text {air }}\right) \frac{2 \pi}{h_{\text {core }} \ln \left(r_{\text {out }} / r_{\text {in }}\right)}+\mu_{0}, \\
& \mu_{\mathrm{s}}^{\prime \prime}=\left(R_{\mathrm{s}}-R_{\text {cav }}\right) \frac{2 \pi}{\omega h_{\text {core }} \ln \left(r_{\text {out }} / r_{\text {in }}\right)}
\end{aligned}
$$




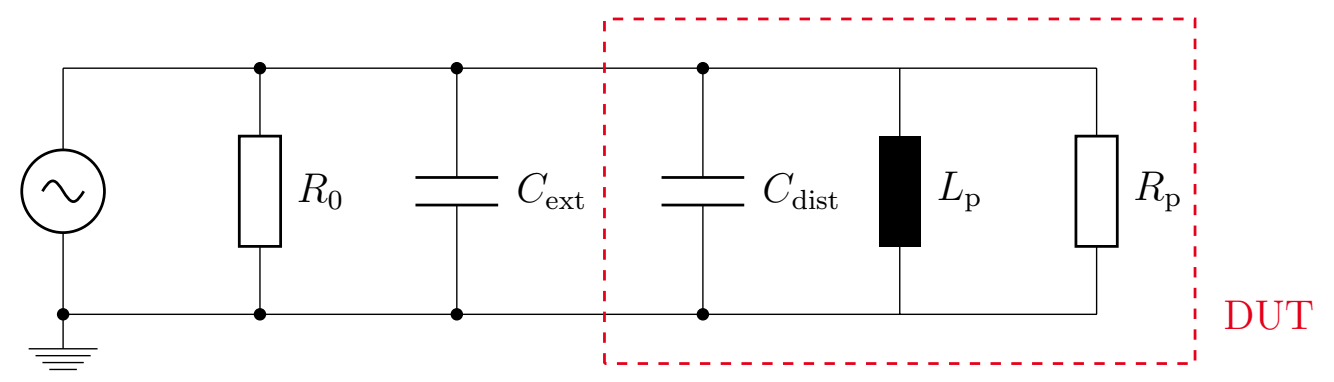

Figure 2.8.: Alternative equivalent circuit diagram for the measurement setup. The resistor $R_{0}$ and optional external capacitors $C_{\text {ext }}$ are installed in parallel to the DUT. The DUT itself is modeled as a distributed capacitance $C_{\text {dist }}$ in parallel with a parallel inductance $L_{\mathrm{p}}$ and resistance $R_{\mathrm{p}}$.

with $L_{\mathrm{s}}$ and $R_{\mathrm{s}}$ as given in equations (2.48). Equation (2.51) allows only a rather inaccurate determination of the inductance of the empty cavity in practice. Hence, the inductance $L_{\text {air }}$ and also the resistance $R_{\text {cav }}$ of the empty cavity are obtained instead from a separate measurement without the toroids as explained in subsection 2.3.2.2. Furthermore, the distributed capacitance remains unknown at this point. Its estimation is discussed in subsection 2.3.2.3.

For certain purposes it may be more convenient to define a complex parallel permeability

$$
\frac{1}{\mu_{\mathrm{p}}}=\frac{1}{\mu_{\mathrm{p}}^{\prime}}+\mathrm{i} \frac{1}{\mu_{\mathrm{p}}^{\prime \prime}}
$$

and to work with an alternative circuit model with a parallel inductance $L_{\mathrm{p}}$ and resistance $R_{\mathrm{p}}$ as depicted in figure 2.8. In this case, $L_{\mathrm{p}}$ and $R_{\mathrm{p}}$ are related to the admittance by

$$
\begin{aligned}
L_{\mathrm{p}} & =\frac{1}{\omega\left(-\operatorname{Im}(Y)+\operatorname{Im}\left(Y_{0}\right)+\omega C_{\mathrm{dist}}\right)}, \\
R_{\mathrm{p}} & =\frac{1}{\operatorname{Re}(Y)-\operatorname{Re}\left(Y_{0}\right)}
\end{aligned}
$$

respectively. The derivation of expressions for $L_{\mathrm{p}}$ and $R_{\mathrm{p}}$ in terms of geometric parameters is completely analogous to equations (2.49) and (2.50). The final result thus reads

$$
\begin{aligned}
& \mu_{\mathrm{p}}^{\prime}=\left(L_{\mathrm{p}}-L_{\text {air }}\right) \frac{2 \pi}{h_{\text {core }} \ln \left(r_{\text {out }} / r_{\text {in }}\right)}+\mu_{0}, \\
& \mu_{\mathrm{p}}^{\prime \prime}=\left(R_{\mathrm{p}}-R_{\text {cav }}\right) \frac{2 \pi}{\omega h_{\text {core }} \ln \left(r_{\text {out }} / r_{\text {in }}\right)}
\end{aligned}
$$

with $L_{\mathrm{p}}$ and $R_{\mathrm{p}}$ as given in equations (2.54).

Besides of the values for the permeability, also the dependence of the lowest resonance frequency of the whole system on the bias field is of interest. The relation between the bias current and the applied magnetic bias field $H_{\text {bias }}$ can be expressed as [41]

$$
H_{\text {bias }}=\frac{N_{\text {bias }} I}{l_{\text {eff }}}
$$


with the effective magnetic path length $l_{\text {eff }}$, which is usually specified by the manufacturer. For simple toroidal shapes, $l_{\mathrm{eff}}$ is calculated as [42]

$$
l_{\mathrm{eff}}=\frac{2 \pi \ln \left(r_{\mathrm{out}} / r_{\mathrm{in}}\right)}{1 / r_{\text {in }}-1 / r_{\mathrm{out}}},
$$

which is also supposed throughout the following analysis. Later on, also the effect of using alternative expressions for $l_{\text {eff }}$, namely the arithmetic mean

$$
l_{\mathrm{eff}}=\pi\left(r_{\mathrm{out}}+r_{\mathrm{in}}\right)
$$

or the geometric mean

$$
l_{\text {eff }}=2 \pi \sqrt{r_{\text {out }} r_{\text {in }}}
$$

is investigated with the help of simulations in section 4.2 .

\subsubsection{Measurement of the inductance $L_{\text {air }}$ and the resistance $R_{\text {cav }}$ of the empty cavity}

In order to determine the inductance $L_{\text {air }}$ and the resistance $R_{\text {cav }}$ from measurement, the admittance $Y_{\text {air }}$ of the empty cavity without the ferrite ring cores is recorded. Its real and imaginary part are then fitted to the function

$$
Y_{\mathrm{air}}=\frac{1}{R_{0}}+\frac{1}{\mathrm{i} \omega L_{\mathrm{air}}+R_{\mathrm{cav}}}
$$

keeping $L_{\text {air }}$ and $R_{\text {cav }}$ as free parameters. As can be seen in figure 2.9, it is possible to reproduce $Y_{\text {air }}$ very well with frequency-independent values of $L_{\text {air }}=(180 \pm 8) \mathrm{nH}$ and $R_{\text {cav }}=(17.5 \pm$ 17.5) $\mathrm{m} \Omega$. The uncertainty estimation is discussed in detail in subsection 2.3.3.1.

As a cross-check, the value of $L_{\text {air }}$ is also calculated according to equation (2.51), its uncertainty according to quadratic error propagation with the estimated uncertainties $\Delta h_{\text {cav }}=3 \mathrm{~mm}$, $\Delta r_{\text {cav }, \text { out }}=5 \mathrm{~mm}$ and $\Delta r_{\text {cav,in }}=0.2 \mathrm{~mm}$ for the input quantities. This way a value of $L_{\text {air }}=$ $(151 \pm 7) \mathrm{nH}$ is found, which is not fully in accordance with the measured value. Possible reasons for the slight deviation are additional inductive contributions such as those from the inner wire and the cavity housing, which are not included in the simple calculation. Moreover, an estimation of the resistance $R_{\text {cav }}$ from an analytical calculation taking into account the skin depth of the copper material leads to a compatible value in the same order of magnitude as obtained above.

\subsubsection{Determination of the distributed capacitance}

The distributed capacitance depends only weakly on frequency in good approximation [34] since the permittivity of the ferrite material does not change significantly in the frequency range of this measurement [41]. Thus, the distributed capacitance is assumed to be frequency independent in the following. To estimate its value, the approach suggested in [34] is pursued: There the authors propose to measure the resonance frequency of the DUT together with two 

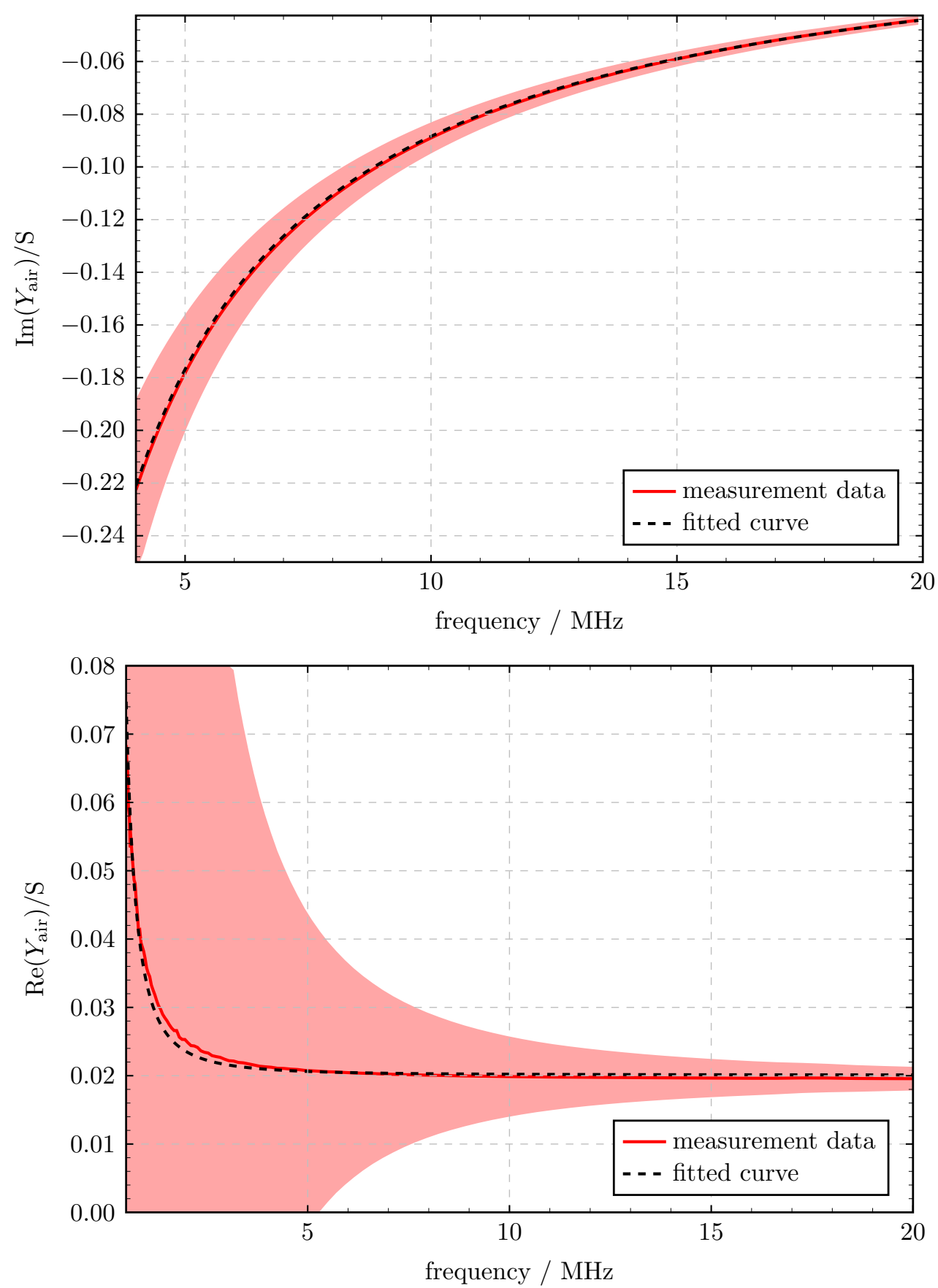

Figure 2.9.: Imaginary part (above) and real part (below) of the admittance $Y_{\text {air }}$ of the empty cavity as a function of frequency for the reflection measurement. The area in light red color indicates the confidence interval $Y_{\text {air }} \pm \Delta Y_{\text {air }}$. The uncertainty of the admittance $\Delta Y_{\text {air }}$ is estimated as discussed in section 2.3.3.1. The fitted curve (black dashed curve) is shown for $L_{\text {air }}=180 \mathrm{nH}$ and $R_{\text {cav }}=17.5 \mathrm{~m} \Omega$ as obtained by a fit to equation (2.60). 
Table 2.2.: (a) Measured capacitances for four different external capacitors at the resonance frequencies $f_{\text {res }}$. (b) Calculated values for the distributed capacitance $C_{\text {dist }}$ according to equation (2.61) for the combination of the data in table (a) for different external capacitors.

(a)

\begin{tabular}{c|cl}
\hline \hline$\#$ & capacitance $/ \mathrm{nF}$ & $@\left(f_{\text {res }} / \mathrm{MHz}\right)$ \\
\hline 1 & $1.98 \pm 0.06$ & $@(1.88 \pm 0.02)$ \\
2 & $8.3 \pm 0.4$ & $@(0.970 \pm 0.004)$ \\
3 & $10.2 \pm 0.5$ & $@(0.881 \pm 0.003)$ \\
4 & $11.2 \pm 0.6$ & $@(0.842 \pm 0.003)$ \\
\hline \hline
\end{tabular}

(b)

\begin{tabular}{c|l}
\hline \hline combination & \multicolumn{1}{|c}{$C_{\text {dist }} / \mathrm{nF}$} \\
\hline 1,2 & $0.31 \pm 0.17$ \\
2,3 & $0.34 \pm 0.17$ \\
3,4 & $0.5 \pm 2.9$ \\
1,3 & $0.34 \pm 0.17$ \\
1,4 & $0.34 \pm 0.17$ \\
2,4 & $0.4 \pm 2.2$ \\
\hline \hline
\end{tabular}

different external capacitors. For an accurate determination of $C_{\text {dist }}$, their capacitance should be chosen close to each other and in the same order as the distributed capacitance. Additionally, they should be selected such that the resonance frequency is well below magnetic resonance, where the inductance of the ferrites is approximately frequency independent. Then, $C_{\text {dist }}$ can be calculated from the two measured resonance frequencies $f_{\text {res, } 1}$ and $f_{\text {res,2 } 2}$ and the known capacitances $C_{1}$ and $C_{2}$ of the capacitors according to the formula [34]

$$
C_{\mathrm{dist}}=\frac{C_{2} f_{\mathrm{res}, 2}^{2}-C_{1} f_{\mathrm{res}, 1}^{2}}{f_{\mathrm{res}, 1}^{2}-f_{\mathrm{res}, 2}^{2}} .
$$

In the elaborated setup, this method is employed using four different external capacitors. At first, their capacitances have to be determined. To this end, the admittance $Y_{0}$ of the capacitors in parallel with a $50 \Omega$-resistor alone is measured separately. The frequency-dependent value of the capacitance can then be calculated from equation (2.47). The obtained capacitances together with the resonance frequencies are listed in table 2.2(a). Moreover, the values for $C_{\text {dist }}$ computed with equation (2.61) for different combinations of external capacitors are summarized in table 2.2(b). Both tables also include uncertainties, which are obtained by considering the extreme cases $\operatorname{Im}(Y) \pm \Delta \operatorname{Im}(Y)$ and $\operatorname{Im}\left(Y_{0}\right) \pm \Delta \operatorname{Im}\left(Y_{0}\right)$, where $\Delta \operatorname{Im}(Y)$ is the estimated error of $\operatorname{Im}(Y)$. Then the uncertainty of the distributed capacitance $\Delta C_{\text {dist }}$ can be estimated according to quadratic error propagation applied to equation (2.61).

As is evident from table 2.2(b), the employed method allows only a very inaccurate determination of the distributed capacitance for this setup. This is due to the fact that here it is not possible to attain the two above-mentioned competing goals simultaneously: On the one hand, a high $C_{\text {ext }}$ is required to shift the resonance to the range of an approximately frequencyindependent $\mu^{\prime}$. On the other hand, a low $C_{\text {ext }}$ is necessary to be in the order of $C_{\text {dist }}$ to avoid cancellation in equation (2.61).

Therefore, a cross-check on $C_{\text {dist }}$ is carried out by determining the slope of the admittance curve $\operatorname{Im}\left(Y-Y_{0}\right)$ in the high frequency range. It is found that it corresponds to that of an ideal capacitor with a capacitance of $C=0.23 \mathrm{nF}$, which might be attributed to distributed capacitances. Yet, as the increasing raise of the $\operatorname{Im}(Y)$-curve towards high frequencies in figure 2.10 suggests, this value might be influenced by parasitic resonances at higher frequencies. All in all, the determined value for the distributed capacitance appears to be unreasonably large. To 


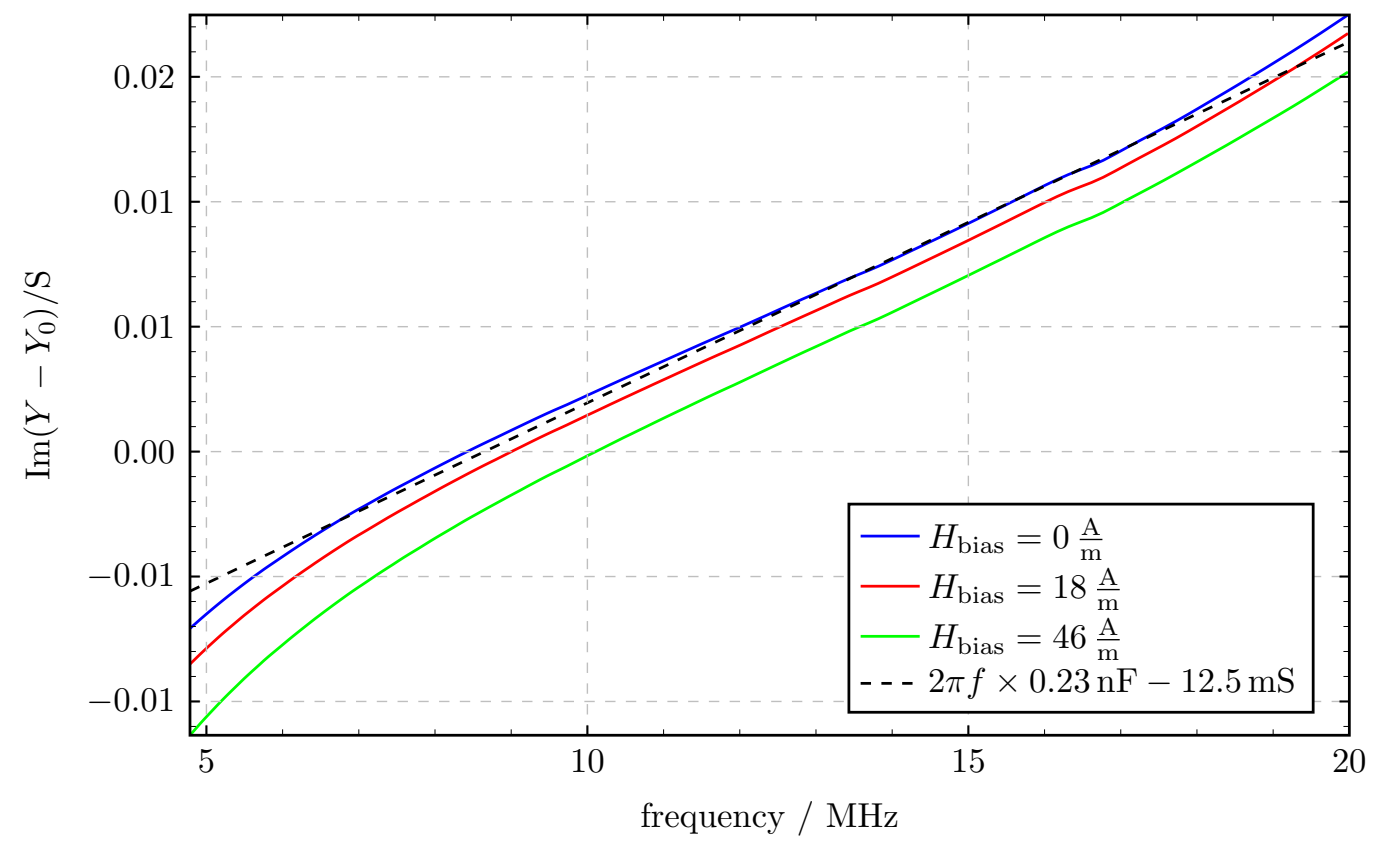

Figure 2.10.: Imaginary part of the admittance $Y-Y_{0}$ as a function of frequency for the reflection measurement setup without external capacitors using three different bias magnetic field strengths. The slope of the admittance curves in the high frequency range corresponds to that of an ideal capacitor with $C=0.23 \mathrm{nF}$, which is indicated by the dashed line.

further investigate the origin of the distributed capacitance, measurements were also performed with a second, completely closed cavity housing, which only takes one single toroid without bias windings. Besides from that, the setup and measurement approach are identical. Nevertheless, the two different methods for the determination of $C_{\text {dist }}$ yield almost identical values. Hence, it cannot be assumed that the large value of $C_{\text {dist }}$ is due to the bias current windings. Furthermore, also the comparison with the eigenmode simulation similar to the one in section 4.2 indicates that stray capacitances between the ring cores and the surrounding cannot add up to such large values. Such numerical eigenvalue simulations of the cavity resonator loaded with two ring cores of a material with a spatially constant as well as frequency independent permeability and a permittivity in the range as stated in the data sheet for the Ferroxcube material [41] rather suggest a stray capacitance in the order of $0.01 \mathrm{nF}$. It might thus be assumed that the main contribution to the parasitic capacitance is due to the connector on top of the resonator. However, from the analysis of measurements of the empty cavity with and without additional connectors, the order of magnitude of such a connector is estimated as only $0.01 \mathrm{nF}$. Hence, though the origin of apparent large value of $C_{\text {dist }}$ cannot conclusively be clarified, the influence of parasitic resonances at higher frequencies still seems to be plausible. Consequently, the large values of the distributed capacitance are discarded for the data analysis.

Taking all this into consideration, the value of the distributed capacitance is taken as $C_{\text {dist }}=$ $(0.01 \pm 0.17) \mathrm{nF}$ for the further data evaluation. It is noted that the actual value of $C_{\text {dist }}$ plays only an important role for high frequencies. In the frequency range relevant for the simulation of the SIS 18 cavity even a change of $C_{\text {dist }}$ from $0.23 \mathrm{nF}$ to $0.01 \mathrm{nF}$ for the determination of the permeability values results in changes of the simulated resonance frequency only in the order of $1 \%$. 


\subsubsection{Uncertainty estimation for the reflection measurement}

\subsubsection{Error calculation}

The uncertainty of derived quantities, here in particular the permeability, is estimated under the assumption of uncorrelated variables using quadratic error propagation. Accordingly, starting from equations (2.52) the uncertainty of the permeability $\Delta \mu_{\mathrm{s}}$ is given by

$$
\begin{aligned}
\left(\Delta \mu_{\mathrm{s}}^{\prime}\right)^{2}= & \left(\frac{\partial \mu_{\mathrm{s}}^{\prime}}{\partial \operatorname{Re}(Y)}\right)^{2}\left((\Delta \operatorname{Re}(Y))^{2}+\left(\Delta \operatorname{Re}\left(Y_{0}\right)\right)^{2}\right) \\
& +\left(\frac{\partial \mu_{\mathrm{s}}^{\prime}}{\partial \operatorname{Im}(Y)}\right)^{2}\left((\Delta \operatorname{Im}(Y))^{2}+\left(\Delta \operatorname{Im}\left(Y_{0}\right)\right)^{2}\right) \\
& +\left(\frac{\partial \mu_{\mathrm{s}}^{\prime}}{\partial C_{\text {dist }}}\right)^{2}\left(\Delta C_{\text {dist }}\right)^{2}+\left(\frac{\partial \mu_{\mathrm{s}}^{\prime}}{\partial \omega}\right)^{2}(\Delta \omega)^{2}+\left(\frac{\partial \mu_{\mathrm{s}}^{\prime}}{\partial h_{\text {core }}}\right)^{2}\left(\Delta h_{\text {core }}\right)^{2} \\
& +\left(\frac{\partial \mu_{\mathrm{s}}^{\prime}}{\partial r_{\text {out }}}\right)^{2}\left(\Delta r_{\text {out }}\right)^{2}+\left(\frac{\partial \mu_{\mathrm{s}}^{\prime}}{\partial r_{\text {in }}}\right)^{2}\left(\Delta r_{\text {in }}\right)^{2}+\left(\frac{\partial \mu_{\mathrm{s}}^{\prime}}{\partial L_{\text {air }}}\right)^{2}\left(\Delta L_{\text {air }}\right)^{2}
\end{aligned}
$$

and an analogous expression for $\Delta \mu_{\mathrm{s}}^{\prime \prime}$. Of course, the equations for the calculation of the uncertainty of the permeability in the parallel equivalent circuit representation $\Delta \mu_{\mathrm{p}}$ are identical apart from the fact that the index $s$ has to be replaced with $\mathrm{p}$. The geometrical uncertainties $\Delta h_{\text {core }}, \Delta r_{\text {out }}$ and $\Delta r_{\text {in }}$ are estimated from repeated measurements at various locations of the toroids. In contrast, the errors of the admittance $\Delta \operatorname{Re}(Y)$ and $\Delta \operatorname{Im}(Y)$ as well as the one of the frequency $\Delta \omega$ are governed by the specifications of the network analyzer. Note that since the admittance $Y$ is actually a derived quantity, only the uncertainties of the absolute value and phase of the complex $S_{11}$-parameter are known a priori. To obtain an error estimate for $Y$ (and analogously for $Y_{0}$ ), the relations (2.45) together with

$$
\begin{aligned}
& \Delta \operatorname{Re}(Y)=\sqrt{\left(\frac{\partial \operatorname{Re}(Y)}{\partial\left|S_{11}\right|}\right)^{2}\left(\Delta\left|S_{11}\right|\right)^{2}+\left(\frac{\partial \operatorname{Re}(Y)}{\partial \arg \left(S_{11}\right)}\right)^{2}\left(\Delta \arg \left(S_{11}\right)\right)^{2}}, \\
& \Delta \operatorname{Im}(Y)=\sqrt{\left(\frac{\partial \operatorname{Im}(Y)}{\partial\left|S_{11}\right|}\right)^{2}\left(\Delta\left|S_{11}\right|\right)^{2}+\left(\frac{\partial \operatorname{Im}(Y)}{\partial \arg \left(S_{11}\right)}\right)^{2}\left(\Delta \arg \left(S_{11}\right)\right)^{2}}
\end{aligned}
$$

are used. The uncertainties $\Delta\left|S_{11}\right|, \Delta \arg \left(S_{11}\right)$ and $\Delta \omega$ as specified by the manufacturer are available in [43]. Furthermore, the estimation of the uncertainty of the distributed capacitance $\Delta C_{\text {dist }}$ was already discussed in subsection 2.3.2.3. The one of the inductance and resistance of the empty cavity determined in subsection 2.3.2.2 is estimated as follows. As revealed by the above uncertainty analysis, $Y_{\text {air }}$ can be measured with highest accuracy in the high frequency limit (cf. Fig. 2.9). This suggests to repeat the fitting procedure mentioned in subsection 2.3.2.2, however, this time with $Y_{\text {air }} \pm \Delta Y_{\text {air }}$ and only in the high frequency range near $20 \mathrm{MHz}$ in order to obtain confidence intervals for $L_{\text {air }}$ and $R_{\text {cav }}$. Due to the observed frequency independence of $L_{\text {air }}$ and $R_{\text {cav }}$, the uncertainties obtained this way are assumed to be valid in the whole frequency range. For the resistance $R_{\text {cav }}$, the estimated uncertainty is reduced to ensure manifestly positive values. The assumed uncertainties of all input quantities are summarized in table 2.3. 
Table 2.3.: Assumed uncertainties for the input quantities. The symbols used for the different quantities are explained in the text.

\begin{tabular}{c|l}
\hline \hline Quantity & Value \\
\hline$\Delta\left|S_{11}\right|$ & as depicted at the top right \\
$\Delta \arg \left(S_{11}\right)$ & as depicted on the bottom right \\
$\Delta C_{\text {dist }}$ & $0.17 \mathrm{nF}$ \\
$\Delta L_{\text {air }}$ & $10 \mathrm{nH}$ \\
$\Delta R_{\text {cav }}$ & $17.5 \mathrm{~m} \Omega$ \\
$\Delta \omega$ & $0 \mathrm{~Hz}\left(\right.$ according to $\left.[43] \mathscr{O}\left(10^{-6}\right) \times \omega\right)$ \\
$\Delta h_{\text {core }}$ & $0.6 \mathrm{~mm}$ \\
$\Delta r_{\text {out }}$ & $0.5 \mathrm{~mm}$ \\
$\Delta r_{\text {in }}$ & $1 \mathrm{~mm}$ \\
$\Delta r_{\mathrm{I}, \text { out }}$ & $6 \mathrm{~mm}$ \\
$r_{\mathrm{I}, \text { in }}$ & $6 \mathrm{~mm}$ \\
$\Delta h_{\mathrm{I}}$ & $5 \mathrm{~mm}$ \\
$\Delta I$ & $0.5 \% \times I+0.02 \mathrm{~A}$ \\
\hline \hline
\end{tabular}
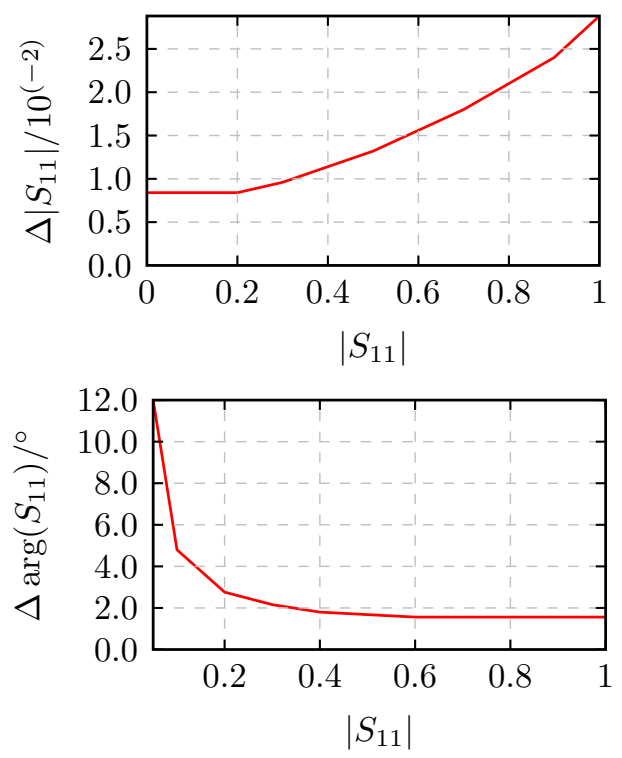

Moreover, the uncertainty of the position of the lowest resonance of the system as a function of frequency and bias magnetic field is estimated as follows. The uncertainty of the bias magnetic field strength is calculated according to

$$
\Delta H_{\text {bias }}=\sqrt{\left(\frac{\partial H_{\text {bias }}}{\partial I}\right)^{2}(\Delta I)^{2}+\left(\frac{\partial H_{\text {bias }}}{\partial r_{\text {out }}}\right)^{2}\left(\Delta r_{\text {I,out }}\right)^{2}+\left(\frac{\partial H_{\text {bias }}}{\partial r_{\text {in }}}\right)^{2}\left(\Delta r_{\text {I,in }}\right)^{2}} .
$$

Here the uncertainty of the bias current $\Delta I$ is taken as specified by the manufacturer of the power supply [31]. Furthermore, somewhat larger uncertainties for the inner and outer radius, $\Delta r_{\text {I,in }}$ and $\Delta r_{\text {I,out }}$, are assumed since here the radii of the current windings instead of those of the toroids matter. These uncertainties are also listed in table 2.3. At this point, errors due to the approximating assumption of a constant effective magnetic field in equation (2.56) are not taken into account. The uncertainty of the resonance frequency itself is obtained by determining the zeros of the imaginary part of the admittance for the extreme cases $\operatorname{Im}(Y) \pm \Delta \operatorname{Im}(Y)$.

Note that any possible other errors, in particular ones due to temperature variations, are not considered. Furthermore, it is worth mentioning again that possible correlations of variables, notable that of $\Delta \operatorname{Re}(Y)$ and $\Delta \operatorname{Im}(Y)$, are not taken into account, which, however, only might lead to an overestimation of uncertainties.

\subsubsection{Discussion of individual uncertainty contributions}

The estimated relative error for the determination of the lowest resonance frequency is fairly below $1 \%$ since the uncertainty of $\operatorname{Im}(Y)$ is small in this region. In contrast, the total estimated error for the permeability may become very large. To better understand its origin, the different groups of uncertainties introduced in the previous subsection are examined separately. The qualitative results are presented in the following starting with the most important contributions. 
If not stated otherwise, the obtained uncertainties are similar both for $\mu_{\mathrm{s}}$ and $\mu_{\mathrm{p}}$; in such a case the symbol $\mu$ is used without index.

\section{Uncertainty of $\left|S_{11}\right|$ and $\arg \left(S_{11}\right)$}

The uncertainty of the complex $S_{11}$-parameter presents one of the largest contributions to the total estimated error. Whereas the caused relative error of $\mu^{\prime}$ is still moderate in the order of $10 \%$, the resulting relative error of $\mu^{\prime \prime}$ rises up to the order of $1000 \%$ for small frequencies. Moreover, in general, the resulting uncertainty of $\mu$ increases significantly when external capacitors are added.

\section{Uncertainty of distributed capacitance}

The effect of the uncertainty of capacitance is negligible for small frequencies but grows rapidly with frequency. This is particularly the case for $\Delta \mu^{\prime}$ as $\mu^{\prime}$ decreases where its relative error exceeds $100 \%$. Yet, for the imaginary part of $\mu$, a distinction of the series and the parallel case is required: Whereas $\mu_{\mathrm{p}}^{\prime \prime}$ is independent of $C_{\mathrm{dist}}$, the resulting relative error $\Delta \mu_{\mathrm{s}}^{\prime \prime} / \mu_{\mathrm{s}}^{\prime \prime}$ is in the order of $100 \%$ around $20 \mathrm{MHz}$. A virtual limit frequency below which an error contribution stays moderate raises as the bias current increases.

\section{Uncertainty of inductance and resistance of the empty cavity}

The uncertainty of $L_{\text {air }}$ only contributes to $\Delta \mu^{\prime}$. Since $\Delta L_{\text {air }}$ is assumed to be independent of frequency (cf. subsection 2.3.2.2) and also independent of the bias field strength, the resulting relative error of $\mu^{\prime}$ raises with increasing frequency and decreasing bias current. It is in the order of $10 \%$ in a wide frequency range. In contrast, the uncertainty of $R_{\text {cav }}$ may be neglected compared to other more dominant contributions.

\section{Uncertainty of geometry}

The uncertainty of the geometry results in a relative error in the order of $1 \%$.

As revealed by the error analysis, the resulting relative error is far above $10 \%$ for some parameter ranges. In these regions the calculated uncertainties can only be considered as rough estimates since higher order terms are not taken into account in the error calculation.

\subsubsection{Analysis of the transmission measurement data}

Within the transmission measurement, the full two-port scattering matrix is recorded. The complex permeability is then extracted as derived in the following.

The variable-frequency harmonic oscillator of the network analyzer drives a current $\underline{I}_{1}(t)=$ $\underline{I}_{1} \exp (i \omega t)$ through the primary winding with $N_{1}$ primary turns, which in turn excites the (complex) magnetic field strength

$$
\underline{H}(t, r)=N_{1} \frac{\underline{I}_{1}(t)}{2 \pi r}
$$




\begin{tabular}{c|c}
\hline \hline Quantity & \multicolumn{1}{|c}{ Value } \\
\hline$h_{\mathrm{I}}$ & $71 \mathrm{~mm}$ \\
$r_{\mathrm{I}, \text { out }}$ & $254 \mathrm{~mm}$ \\
$r_{\mathrm{I}, \text { in }}$ & $131 \mathrm{~mm}$ \\
\hline \hline
\end{tabular}

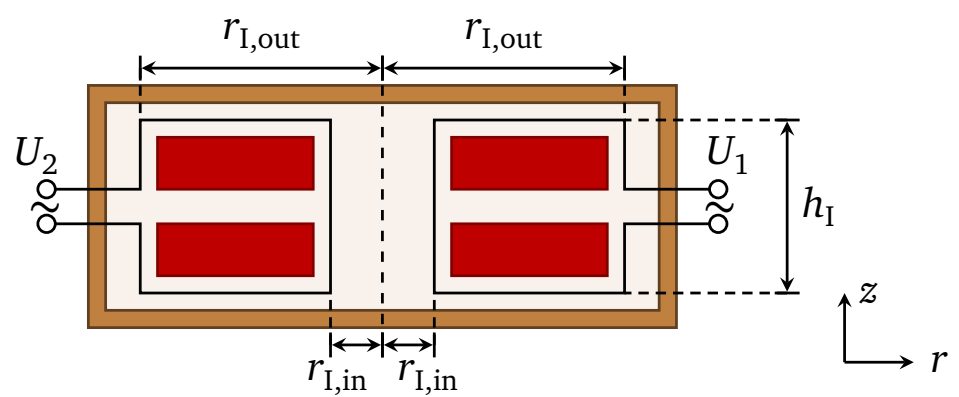

Figure 2.11.: Geometric quantities related to the location of the primary and secondary winding. The illustration on the right shows a simplified two-dimensional sectional view through the center of the cavity. [30]

inside the toroids [38]. The secondary winding with $N_{2}$ turns, which is used as sense winding, comprises the magnetic flux (cf. Fig. 2.11)

$$
\underline{\Phi}(t)=\int \underline{\vec{B}}(t) \mathrm{d} \vec{A}=\int_{r_{\mathrm{I}, \text { in }}}^{r_{\mathrm{I}, \text { out }}}\left[\left(\mu_{\text {core }}-\mu_{0}\right) h_{\text {core }}+\mu_{0} h_{\mathrm{I}}\right] \underline{H}(t, r) \mathrm{d} r=\frac{N_{1} \underline{I}_{1}(t)}{2 \pi}\left(\mu_{\text {core }} h_{\text {core }} \ln \frac{r_{\text {out }}}{r_{\text {in }}}+\Psi_{\text {air }}\right)
$$

$$
\text { with } \quad \Psi_{\text {air }}:=-\mu_{0} h_{\text {core }} \ln \frac{r_{\text {out }}}{r_{\text {in }}}+\mu_{0}\left(h_{\text {core }}+h_{\mathrm{I}}\right) \ln \frac{r_{\mathrm{I}, \text { out }}}{r_{\mathrm{I}, \text { in }}},
$$

where $\mu_{\text {core }}$ is the complex permeability of the ferrite ring cores. Note that the global sign on the right hand side of equation (2.66a) depends on the relative orientation of current and sense winding. By Faraday's law the voltage

$$
\underline{U}_{2}(t)=\underline{U}_{2} \exp (\mathrm{i} \omega t)=-N_{2} \frac{\mathrm{d} \underline{\Phi}(t)}{\mathrm{d} t}=-\mathrm{i} \omega \frac{N_{1} N_{2} \underline{I}_{1}(t)}{2 \pi}\left(\mu_{\text {core }} h_{\text {core }} \ln \frac{r_{\text {out }}}{r_{\text {in }}}+\Psi_{\text {air }}\right)
$$

is induced in the secondary winding. Solving this complex-valued equation for the real and imaginary part of the permeability in the series representation, i.e. $\mu_{\text {core }}:=\mu_{\mathrm{s}}^{\prime}-\mathrm{i} \mu_{\mathrm{s}}^{\prime \prime}$, finally yields

$$
\begin{aligned}
& \mu_{\mathrm{s}}^{\prime}=-\frac{1}{h_{\text {core }} \ln \left(r_{\text {out }} / r_{\text {in }}\right)}\left(\frac{2 \pi \operatorname{Im}\left(Z_{21}\right)}{N_{1} N_{2} \omega}+\Psi_{\text {air }}\right) \\
& \mu_{\mathrm{s}}^{\prime \prime}=-\frac{1}{h_{\text {core }} \ln \left(r_{\text {out }} / r_{\text {in }}\right)} \frac{2 \pi \operatorname{Re}\left(Z_{21}\right)}{N_{1} N_{2} \omega}
\end{aligned}
$$

involving the impedance parameter [40]

$$
Z_{21}:=\left.\frac{\hat{U}_{2}}{\underline{\hat{I}}_{1}}\right|_{I_{2}=0}=\frac{2 S_{21}}{\left(1-S_{11}\right)\left(1-S_{22}\right)-S_{12} S_{21}} Z_{0}
$$

where $Z_{0}=50 \Omega$ is the characteristic impedance. 

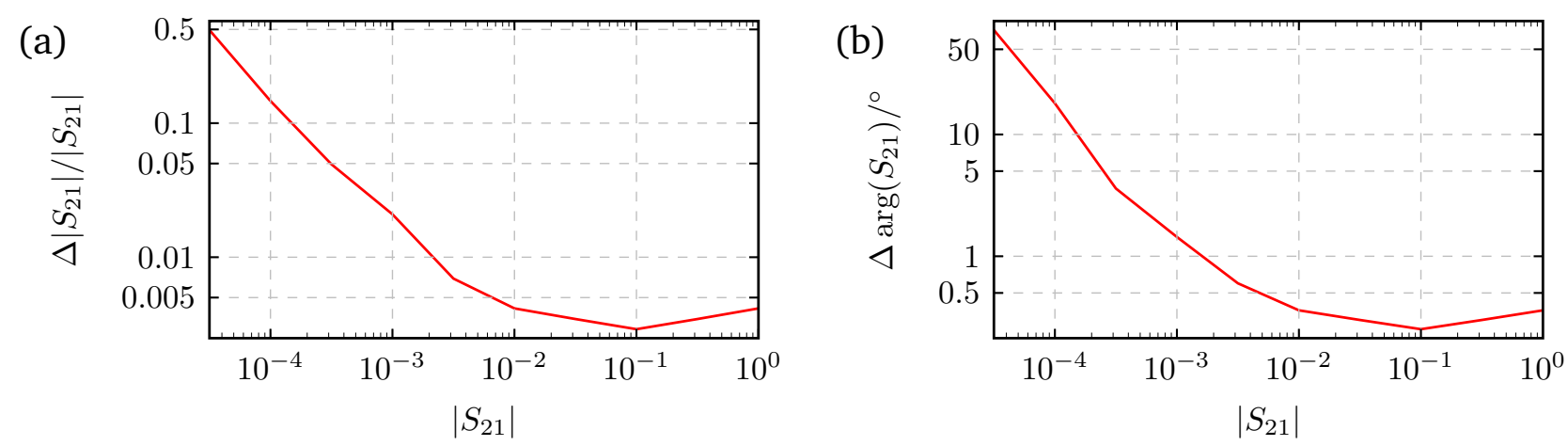

Figure 2.12.: Assumed uncertainties for the transmission parameters $S_{21}$ and $S_{12}$ for the absolute value (a) and the argument (b) following [43]. The same dependence as shown for $S_{21}$ is assumed for $S_{12}$.

\subsubsection{Uncertainty estimation for the transmission measurement}

\subsubsection{Error calculation}

Analogous to the reflection measurement, the uncertainty of the permeability is derived using quadratic error propagation from equations (2.68). The required uncertainty of all geometric parameters as well as the one of $\omega$ were already summarized in table 2.3. The uncertainty of the $Z_{21}$-parameter is derived employing quadratic error propagation from equation (2.69). The assumed uncertainties of the reflection parameters $S_{11}$ and $S_{22}$ therein are as shown for $S_{11}$ in table 2.3, the ones for the transmission parameters $S_{21}$ and $S_{12}$ as indicated in figure 2.12. It is repeated again that possible correlations between variables, notable the one between all the $S$ parameters, are neglected for simplicity. Furthermore, other uncertainties, such as ones due to temperature variations or phase shifts due to residual inductances, are not taken into account.

\subsubsection{Discussion of individual uncertainty contributions}

For a better understanding of the origin of the total estimated uncertainty of the permeability, the contributions of the two considered groups are examined separately. The qualitative results are presented in the following.

\section{Uncertainty of $S$-parameters}

The total estimated error is dominated by the uncertainty of the complex $S$-parameters. The relative error of $\mu_{\mathrm{s}}^{\prime \prime}$ becomes large for small frequencies, where it is in the order of $100 \%$. It gradually decreases with raising frequency up to $\approx 3 \mathrm{MHz}$ and remains at this level beyond that point. In contrast, very moderate relative errors around $5 \%$ are obtained for $\mu_{\mathrm{s}}^{\prime}$.

\section{Uncertainty of geometry}

The dependence of $\mu_{\mathrm{s}}^{\prime \prime}$ on geometric parameters is the same as for the reflection measurement and is on the order of $1 \%$.

The uncertainty estimates should be taken with care in the regions where the resulting relative error is far above $10 \%$ since only first order terms are taken into account in the error calculation. 


\subsubsection{Permeability as a function of frequency and bias magnetic field strength}

\section{Reflection measurement}

The values obtained from the analysis of the reflection measurement data for the real and imaginary part of the permeability in the series representation as a function of frequency for a few selected bias magnetic field strengths are shown in figure 2.13. In the original measurement data, distortions in the real part of the admittance at low frequencies are observed, which result in sharp resonances, where the value of $\mu_{\mathrm{s}}^{\prime \prime}$ is increased and the one of $\mu_{\mathrm{p}}^{\prime \prime}$ is decreased (cf. Fig. 2.13). These distortions are present only for a non-vanishing bias current between $\approx 0.5 \mathrm{MHz}$ and $\approx 2.5 \mathrm{MHz}$. Furthermore, their amplitude firstly increases with bias current, reaches a maximum at around $I=3 \mathrm{~A}\left(H_{\text {bias }} \approx 0.3 \times 10^{3} \mathrm{~A} / \mathrm{m}\right)$ and then gradually decreases. At the same time, the distortions move to higher frequencies. Moreover, the frequency positions and amplitude of the distortions are independent of the number of added external capacitors. It is speculated that they are caused by undesired interactions between the DC current generator and distributed capacitances of the bias current windings. This suspicion is substantiated by the observation that the frequency of the distortions changes when the bias windings are additionally grounded somewhere in between. Exploiting this, these distortions could eventually be eliminated by combining different sets of data which were taken with and without additional grounding. Furthermore, as will be seen in the next paragraph, the same distortions also occur for the transmission measurement, for which, however, no correction has been performed.

Moreover, figure 2.14 demonstrates the dependence of $\mu_{\mathrm{s}}^{\prime}$ and $\mu_{\mathrm{s}}^{\prime \prime}$ on the bias magnetic field strength as a function of frequency. Additionally, similar plots are shown in figure 2.15 for the parallel representation $\mu_{\mathrm{p}}$. As the comparison shows, the real part of the permeability is almost identical in the two representations for low frequencies. In contrast, both the qualitative and the quantitative behavior of the imaginary part are completely different, as expected by the known relations between the two quantities [2].

Generally, the results could be well reproduced after dissembling and restoring the whole setup. In order to check the sensitivity on the RF-level, tests with different settings for the source of the VNA were carried out for the reflection measurements. No significant deviations were observed for a source power between $-20 \mathrm{dBm}$ and $10 \mathrm{dBm}$ except for the increasing noise at very low power levels.

\section{Transmission measurement}

The analysis of the transmission impedance parameter $Z_{21}$ reveals that the measured data are affected by undesired resonances. Experiments demonstrated that the resonance frequency decreases by a factor of approximately $\sqrt{2}$ when doubling the number of sense windings but is rather insensitive with regard to the self inductance of the windings. Such $L C$ resonances are known from literature [38]. In the elaborated setup, the resonance frequency is around $8 \mathrm{MHz}$ without biasing and increases with raising bias current. Nevertheless, an evaluation of the data sufficiently far below the resonance is attempted in the following. To allow an easier comparison of the results from the reflection with the transmission measurement, the obtained values for $\mu_{\mathrm{s}}^{\prime}$ and $\mu_{\mathrm{s}}^{\prime \prime}$ are plotted in figure 2.13 for three selected bias field strengths. As can 


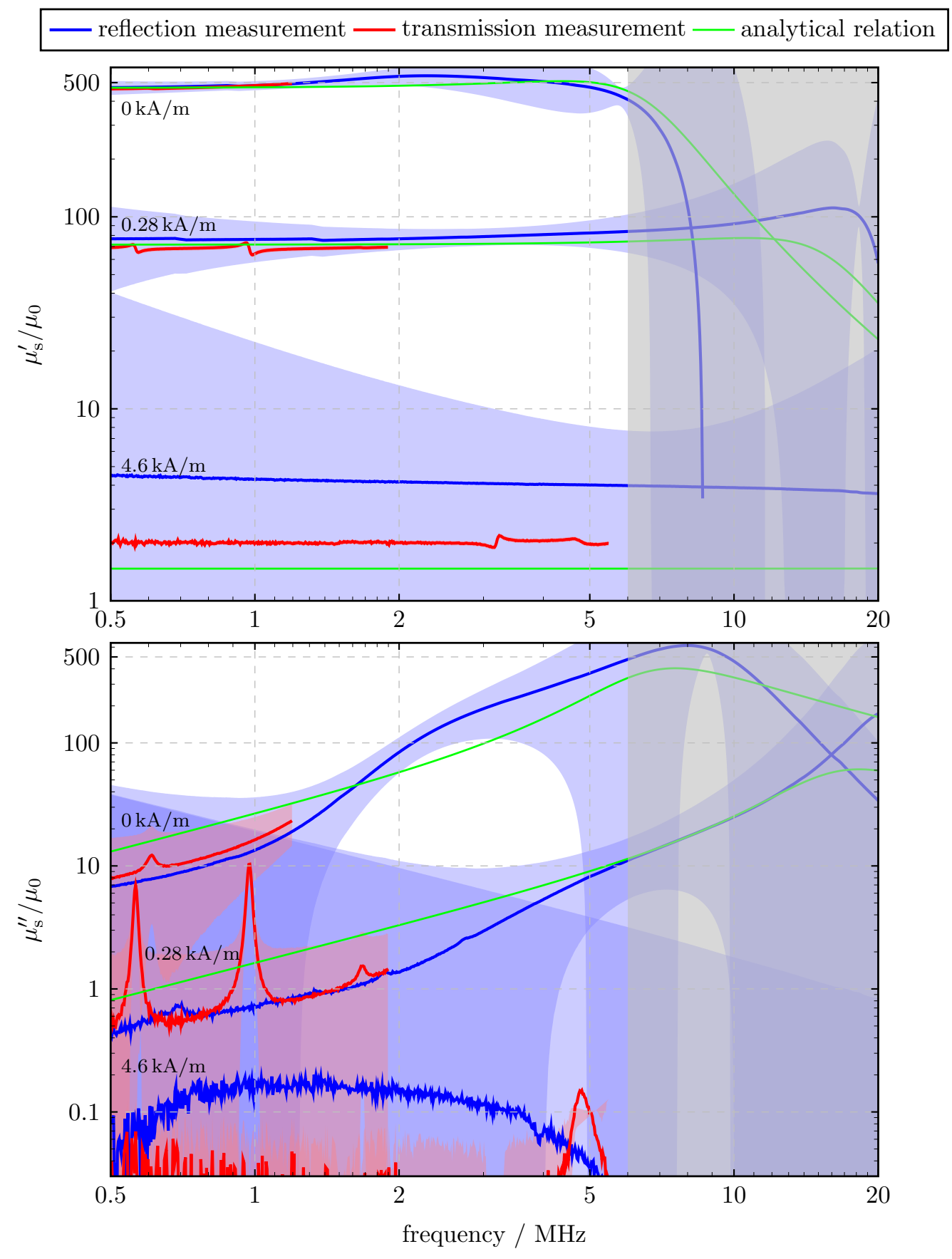

Figure 2.13.: Comparison of the real part (above) and the imaginary part (below) of the permeability $\mu_{\mathrm{s}}$ as obtained from the reflection and transmission measurement analysis for three different bias magnetic field strengths. Regions in lighter color indicate error estimates. Unlike as in the analysis for the reflection setup, no corrections for the distortions, which result in sharp resonances, are applied for the transmission measurement. The empirically found analytical relation (2.70) is also plotted for comparison. The area above $f=6 \mathrm{MHz}$ is grayed out to indicate the decreasing reliability of the measurement results of the employed methods in this frequency range. 

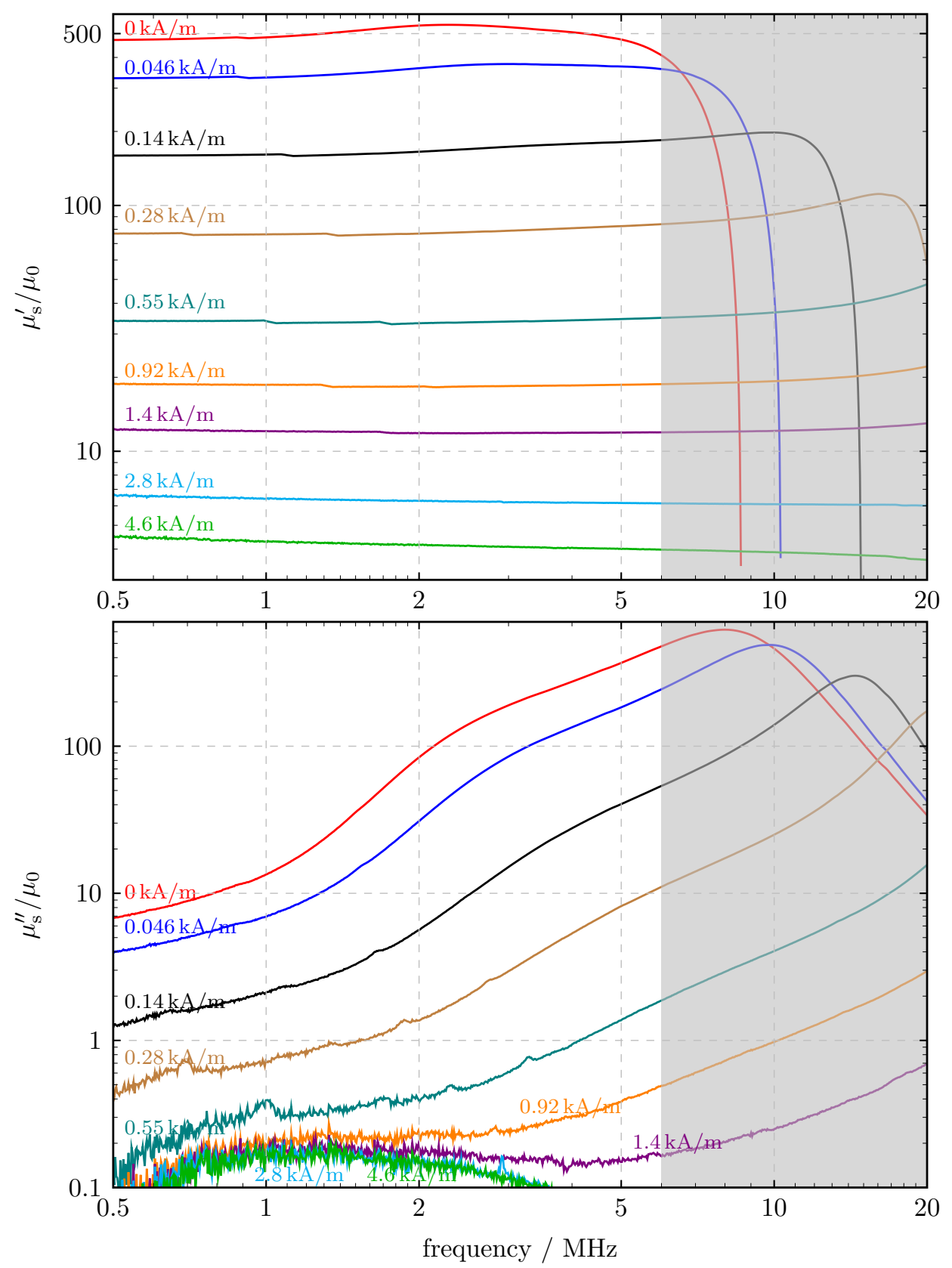

Figure 2.14.: Real part (above) and imaginary part (below) of the permeability $\mu_{\mathrm{s}}$ as obtained from the reflection measurement analysis with different bias field strengths as a function of frequency. The area above $f=6 \mathrm{MHz}$ is grayed out to indicate the decreasing reliability of the measurement results of the employed methods in this frequency range. 


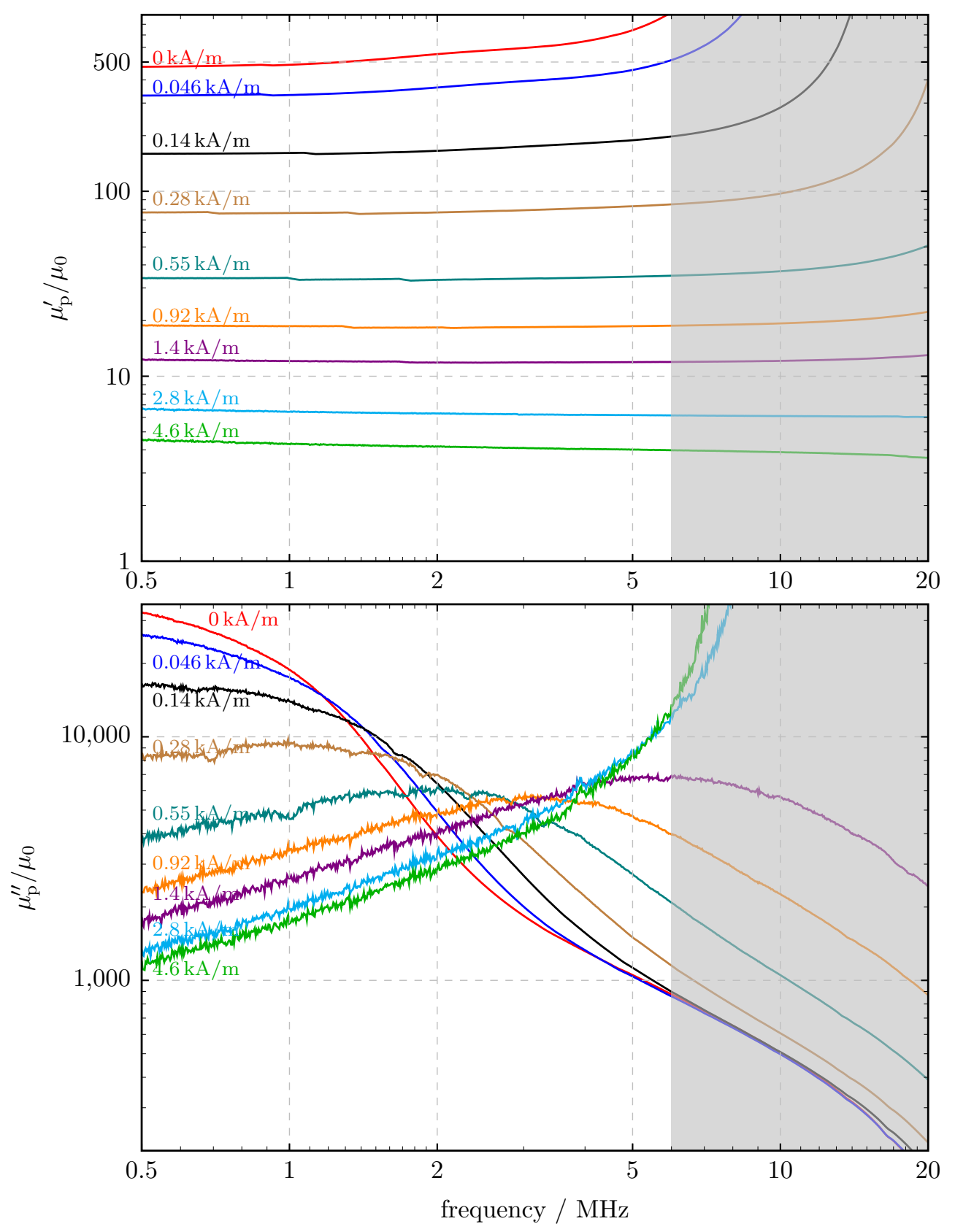

Figure 2.15.: Real part (above) and imaginary part (below) of the permeability $\mu_{\mathrm{p}}$ for the reflection measurement with different bias field strengths as a function of frequency. The area above $f=6 \mathrm{MHz}$ is grayed out to indicate the decreasing reliability of the measurement results of the employed methods in this frequency range. 
be seen there, the values are fully in accordance with each other up in the analyzed frequency region.

The evaluated data presented so far were recorded in a setting where the sense and the primary winding were placed diametrically opposed on the ring cores. In order to check the influence of their relative position, the transmission measurements were repeated with another sense winding, which was installed at an angle of $90^{\circ}$ with respect to the primary winding. The obtained $Z_{21}$-parameters for the two orientations show only minor deviations. More specifically, negligibly small relative deviations in the order of a few percent are observed without bias field. With increasing bias strength and hence decreasing $\mu^{\prime}$, the precise shape of the sense winding plays a more important role. Hence, for a maximal bias current relative deviations up to $20 \%$ are found. To sum up, there is no experimental evidence for a dependence of the measured data on the relative position of the windings.

\section{Description by an empirical analytical relation}

According to the theory discussed in section 2.2, the permeability of ferrites subject to a biasing magnetic field is described by a tensor. Its components involve a couple of material-intrinsic parameters. In this paragraph, a description of the measured permeability values as a function of frequency and magnetic field strength with the help of a relation motivated from theory is attempted. Since the measurements are performed at a variable field strength, the partially magnetized state is the relevant case (cf. subsection 2.2.3.3). Since only information about the dynamic response of the Ferroxcube $8 \mathrm{C} 12 \mathrm{~m}$ material in the direction of the static bias field is available from the performed measurements, only terms for the $\mu_{z}$-component of the permeability tensor are investigated in the following. It is worth recalling that, since $M_{z}=B(H) / \mu_{0}-H$, the dependence of the reduced magnetization $m=M_{z} / M_{\text {sat }}$ on the bias field strength is directly determined by the $B-H$ curve. As discussed in subsection 2.3 .7 , the characteristic function (2.73) is assumed for the Ferroxcube $8 \mathrm{C} 12 \mathrm{~m}$ ferrite. It is then found empirically that the complex permeability values obtained from the reflection measurements for different magnetization states can be well fitted to the relation

$$
\tilde{\mu}_{z}=1+(\hat{\mu}-1) \times\left(1-m^{x}\right)
$$

with $H_{0}=H_{\text {bias }}+H_{a}$ and the permeability of the fully demagnetized state (2.26) without claiming any physical basis. A good approximation of the data up to moderate frequencies is observed for setting the free parameters to $\alpha=0.4, H_{a}=195 \mathrm{~A} / \mathrm{m}$ and $x=1.15$. Equation (2.70) is obtained from the expression for $\widetilde{\mu}_{z}$ in (2.31) with two modifications: First, $\chi=\left(\mu_{\text {diag }}-1\right)$ is substituted with $\hat{\mu}-1$. Second, the exponent $x$ in the spatial averaging (2.30) is left as a free parameter instead of setting it to $x=2$. As discussed above, the ratio $m=M_{z} / M_{\text {sat }}$ directly depends on the $B-H$ function. Thus, the determined value for the exponent is particularly sensitive to a change of the parameters of the characteristic function.

The obtained analytical relations are included in figure 2.13 as well as in figure 2.16 of the following section together with the measurement data.

\subsubsection{Reversible permeability as a function of bias magnetic field strength}

The values for the reversible permeability $\mu_{\mathrm{rev}}$ as a function of bias magnetic field strength as obtained from both measurement methods are shown in figure 2.16. The values are evaluated at a frequency of $f=0.5 \mathrm{MHz}$. 


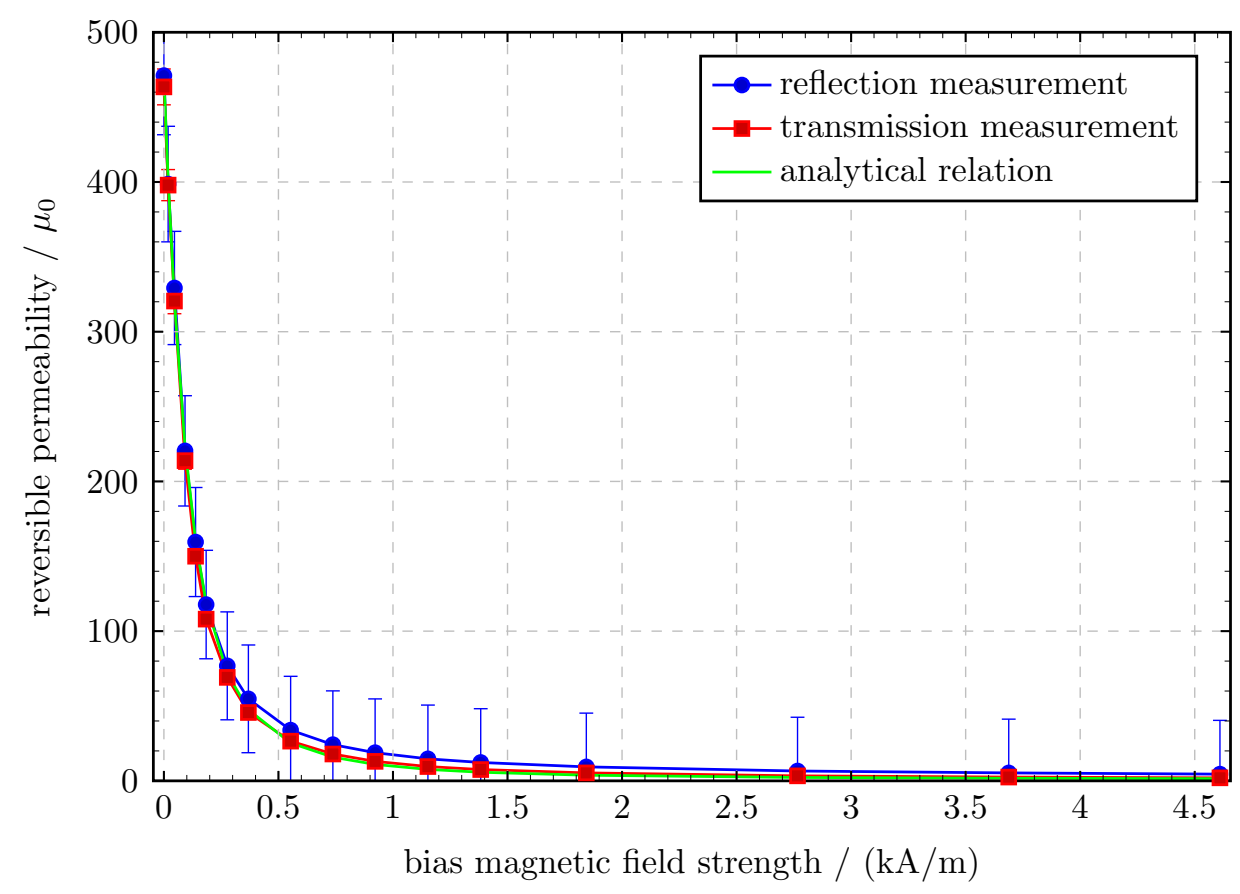

Figure 2.16.: Real part of the reversible permeability in the low frequency limit, in this study evaluated at a frequency of $f=0.5 \mathrm{MHz}$ as a function of the bias magnetic field strength. Both values as obtained for the analysis of the reflection measurement and of the transmission measurement are shown. The empirically found analytical relation (2.70) is also plotted for comparison.

\subsubsection{Product of $\mu Q f$}

As a further figure of merit, also the $\mu_{\mathrm{s}}^{\prime} Q f$-product evaluated for the reflection measurement data for the series representation is shown in figure 2.17. As expected [44], the $\mu Q f$-curve is shifted to the bottom right with increasing bias field strength.

\subsubsection{Measurement of the $B-H$ curve of a working cycle with reduced bias range}

In the working cycle of the GSI SIS 18 cavity, the bias current is modified only in the range from zero to its maximum value without changing its polarity. Hence, not the area of the full $B-H$ loop but only a small part near its upper branch is enclosed. It is the aim of the measurement described in this section to determine the curve surrounding this area by using an approach similar to [38]. The setup which serves for this purpose is as follows. Similar to the previous measurement setup, two full size ferrite ring cores of identical dimensions are biased via $N_{\text {bias }}=24$ crossed (figure-of-eight) current windings. The bias current is again driven by the same DC power supply. Yet, for the $B-H$ loop measurement the output current is controlled by a sinusoidal voltage generated by an Agilent 33522A waveform generator. As a restriction, the maximal effective bias magnetic field was limited to $1.5 \mathrm{kA} / \mathrm{m}$ due to the smaller number of bias windings in this setup. Thus, only a reduced working cycle could be recorded. A voltage monitor output directly proportional to the bias current is connected to the $x$-channel of a LeCroy WaveRunner 44Xi oscilloscope. Taking equation (2.56) into account, this output voltage 


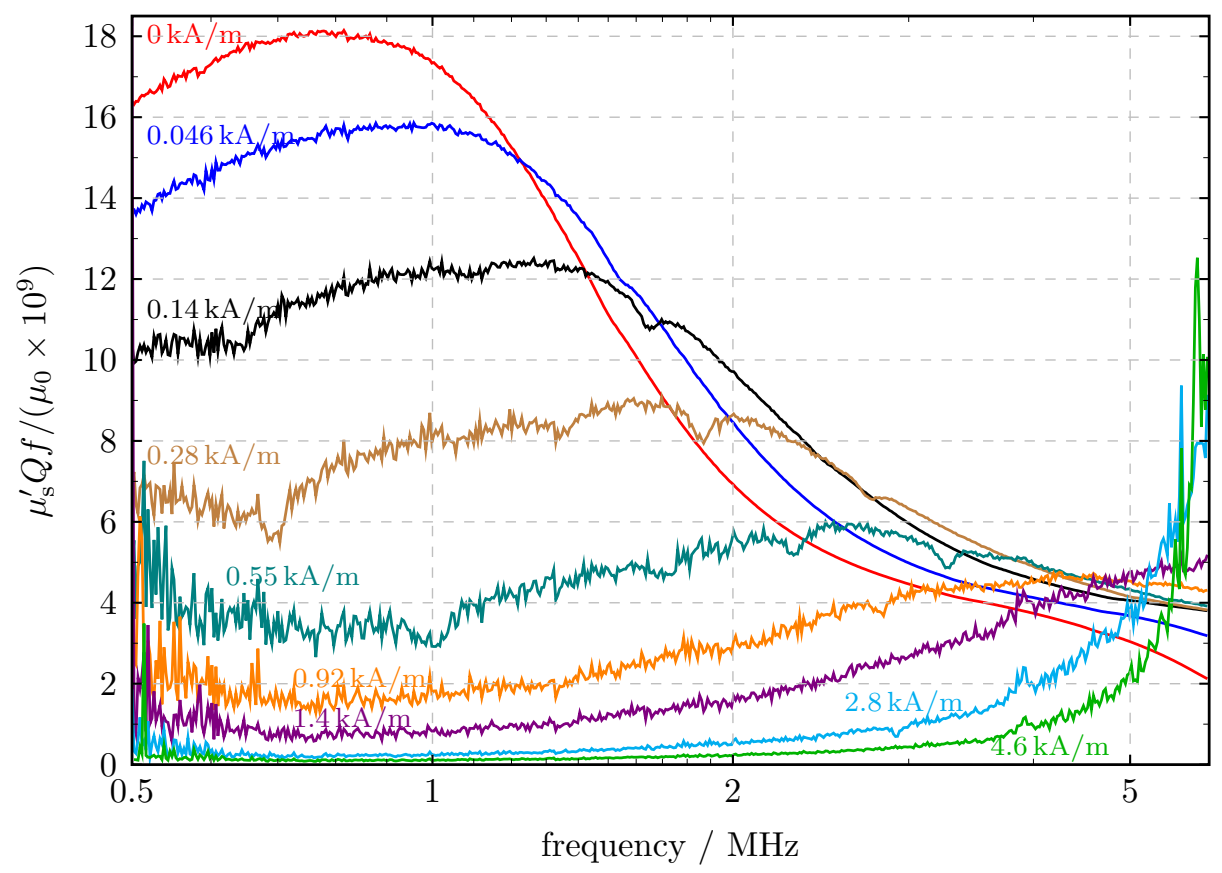

Figure 2.17.: Product of $\mu_{\mathrm{s}}^{\prime} Q f$ for the series representation as obtained from the reflection measurement analysis for different bias field strengths as a function of frequency. [30]

can directly be related to the effective bias magnetic field strength. Moreover, the induced voltage signal from the sense winding with $N_{\text {turns }}=9$ turns is recorded at its $y$-channel and averaged over 50 periods. The induced voltage $U_{\text {ind }}$ is related to the mean magnetic flux density $B$ by

$$
U_{\text {ind }}=N_{\text {turns }} A \frac{\mathrm{d} B}{\mathrm{~d} t},
$$

where $A$ is the area that is penetrated by the magnetic flux. Hence, the magnetic field at the time $t_{n}=t_{n-1}+\Delta t$ can be obtained with numerical integration of the recorded voltage signal as

$$
B\left(t_{n}\right)=\sum_{t_{m}=t_{0}}^{t_{n}} \frac{\Delta t U_{\text {ind }}\left(t_{m}\right)}{N_{\text {turns }}\left(r_{\text {out }}-r_{\text {in }}\right) h_{\text {core }}}+B_{0},
$$

where the inner and outer radius $r_{\text {in }}$ and $r_{\text {out }}$ and the thickness of the ring cores $h_{\text {core }}$ are specified in figure 2.7. The integration constant $B_{0}=B\left(t=t_{0}\right)$ is determined as follows. First of all, a reference value for the saturation magnetization $M_{\text {sat }}$ is required, which is taken as $2.1 \times$ $10^{5} \mathrm{~A} / \mathrm{m}$. This is the mean of the two values stated in the data sheet [41] for a temperature of $25^{\circ} \mathrm{C}$ and $40^{\circ} \mathrm{C}$. Furthermore, as is observed (cf. Fig. 2.18), the area enclosed by the $B-H$ curve is reasonably small, which justifies to neglect hysteresis effects in further considerations. This allows to choose $B_{0}$ such that the resulting saturation magnetization coincides with $M_{\text {sat }}=$ $2.1 \times 10^{5} \mathrm{~A} / \mathrm{m}$ when fitted to a characteristic function of the form [45]

$$
B(H)=\left[a \times \arctan (H b)+H+M_{0}\right] \times \mu_{0}
$$




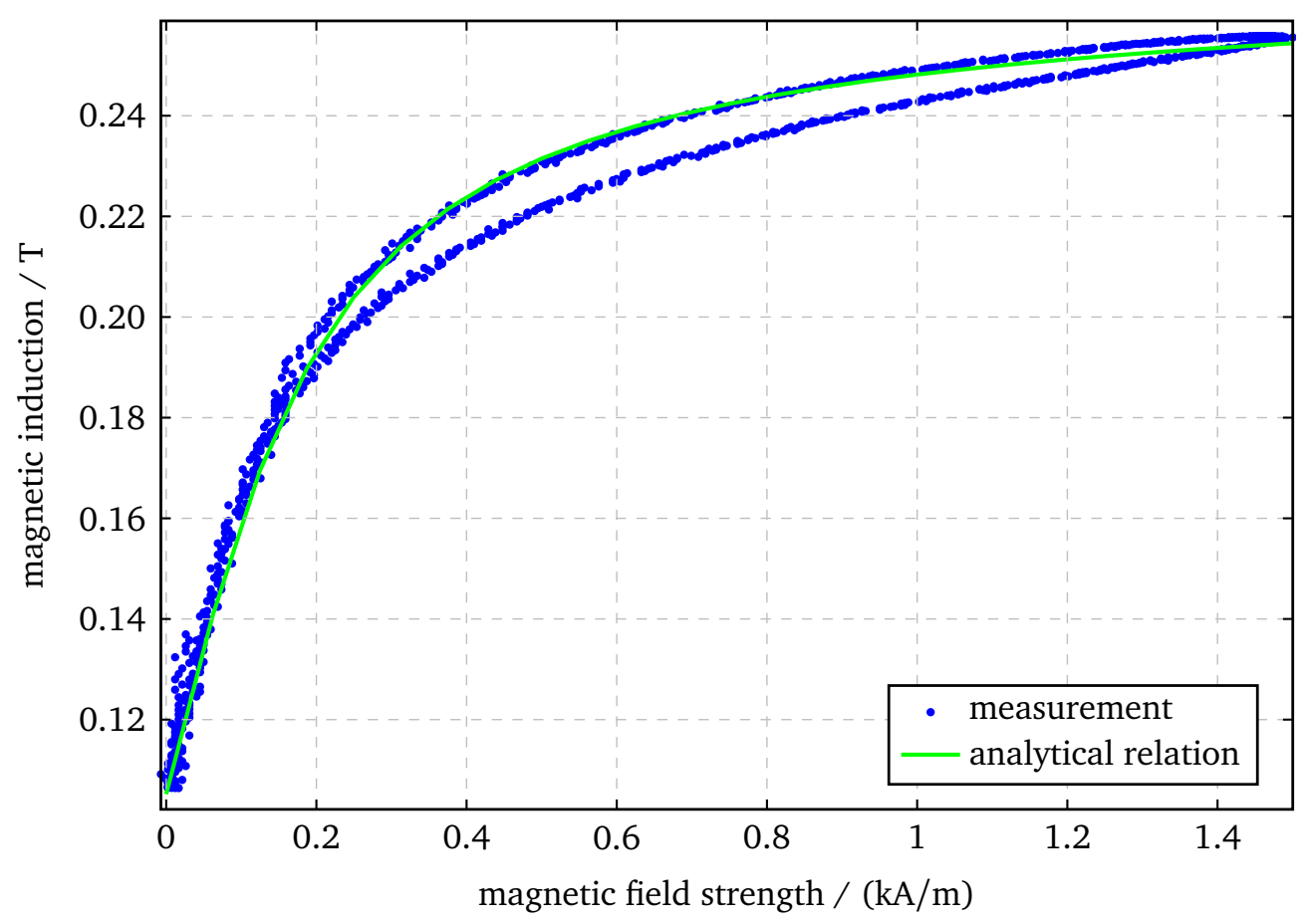

Figure 2.18.: $B-H$ curve for the working cycle with reduced bias range measured at a frequency of $1 \mathrm{~Hz}$. The solid line represents the characteristic functions (2.73) with parameters $a=80.4 \mathrm{kA} / \mathrm{m}, b=0.0059 \mathrm{~m} / \mathrm{A}$ and $M_{0}=83.7 \mathrm{kA} / \mathrm{m}$. [30]

with the free parameters $a, b$ and $M_{0}$. The latter quantity $M_{0}$ may be interpreted as the remanence magnetization. The obtained results for a frequency of $1 \mathrm{~Hz}$ together with equation (2.73) for the parameters $a=80.4 \mathrm{kA} / \mathrm{m}, b=0.0059 \mathrm{~m} / \mathrm{A}$ and $M_{0}=83.7 \mathrm{kA} / \mathrm{m}$ are shown in figure 2.18. This set of parameters will also be used in the numerical simulation in the following section. Moreover, the $B-H$ loop measurements were repeated at the frequencies $5 \mathrm{~Hz}$ and $10 \mathrm{~Hz}$. The obtained curves for all three frequencies are fully in accordance with each other albeit some slight distortions are observed for the measurement at $10 \mathrm{~Hz}$. 


\section{Calculation of eigensystems for materials with fully occupied material tensors}

In this chapter, the basics for the numerical computation of eigenvalues and eigenvectors of systems with fully occupied material tensors as emerging for, e.g., resonators filled with biased ferrites or magnetized plasmas are discussed. First of all, the problem is stated mathematically in terms of the so-called curl-curl equation in section 3.1. After giving an overview of the applied computational model in section 3.2, some basics on distributed computing are reviewed in section 3.3 to facilitate the understanding of remarks on efficient parallel computing throughout the remaining chapter. In section 3.4, the Finite Integration Technique, which is the employed method for the numerical solution of electromagnetic field problems in this thesis, is introduced and extended for gyrotropic materials in frequency domain. The application of this method leads to a large, sparse as well as nonlinear and complex eigenproblem. Numerical methods for the computation of such eigensystems are discussed in section 3.5.

\subsection{Curl-curl equation}

The starting point for the derivation of the governing equation for the description of a resonating electromagnetic field inside a cavity are the two rotational Maxwell's equations (2.1) and (2.2) together with the constitutive relations for the electric and magnetic field. As shown in section 2.2 , these relations for general gyrotropic materials take the form

$$
\begin{aligned}
\vec{H} & =\overleftrightarrow{v} \vec{B}, \\
\vec{D} & =\overleftrightarrow{\varepsilon} \vec{E} .
\end{aligned}
$$

Taking the time-derivative of equation (2.2) for time-harmonic fields $\vec{E}(t)=\underline{\vec{E}} \cdot \exp (\mathrm{i} \omega t), \vec{H}(t)=$ $\underline{\vec{H}} \cdot \exp (i \omega t)$ in the absence of exciting currents, this leads to the system of equations

$$
\begin{aligned}
\nabla \times \underline{\vec{E}} & =-i \omega \stackrel{\leftrightarrow}{v}^{-1} \vec{H}, \\
i \omega \nabla \times \underline{\vec{H}} & =-\omega^{2} \stackrel{\leftrightarrow}{\varepsilon} \underline{\vec{E}} .
\end{aligned}
$$

Inserting equation (3.3) into equation (3.4), yields the so-called curl-curl equation

$$
\overleftrightarrow{\varepsilon}^{-1} \nabla \times(\stackrel{\leftrightarrow}{v} \nabla \times \underline{\vec{E}})=\omega^{2} \underline{\vec{E}} \quad \text { (inside } \Omega \text { ) }
$$

Whereas this equation determines the field inside the resonator $\Omega$, additional conditions have to be imposed for the field on the cavity boundary $\partial \Omega$. For perfect electric cavity walls, the boundary condition takes the form

$$
\vec{n} \times \underline{\vec{E}}=0 \quad(\text { on } \partial \Omega),
$$

where $\vec{n}$ is a normal vector on the cavity boundary $\partial \Omega$. Equation (3.5) has the form of an algebraic eigenvalue equation with the system operator given on its left hand side and the eigenvalue $\omega^{2}$. 


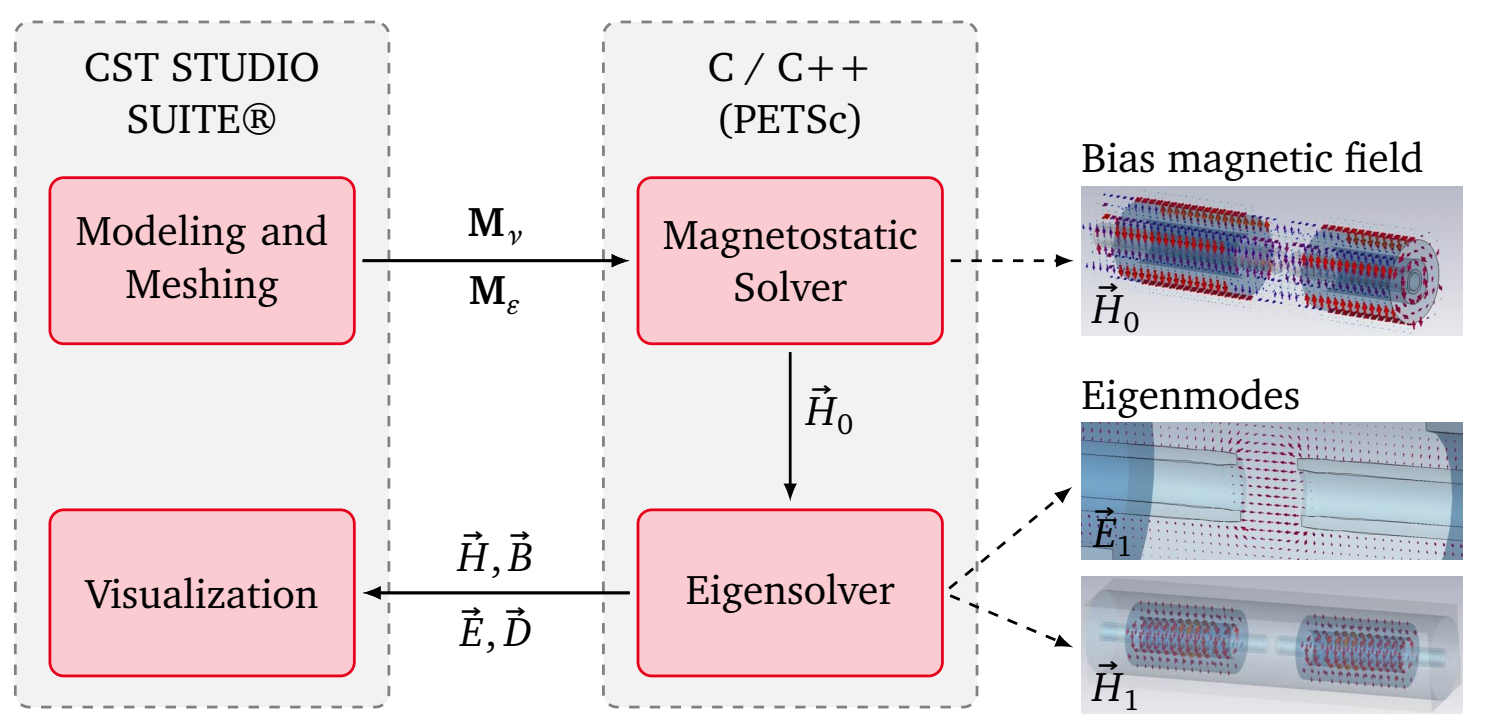

Figure 3.1.: Strategy for the computation of eigenmodes of ferrite-loaded cavity resonators. For Modeling, meshing and visualization the CST STUDIO SUITE ${ }^{[48]}$ is used whereas the newly developed solver is implemented in C / C++ built on PETSC.

\subsection{Computational model: overview}

The computational model for the numerical solution of the eigenvalue problem defined by equation (3.5) and (3.6) is introduced in this section. The need for a dedicated solver arises from the fact that for materials with gyromagnetic properties the inverse permeability tensor is involved on the left hand side of equation (3.5), whereas for gyroelectric materials the permittivity tensor is present. As discussed in section 2.2, the components of both tensors are a function of the static magnetic field as well as of the frequency of the superimposed RF. This has important consequences for the way how the eigensolutions are computed. On the one hand, this implies that the magnetostatic field problem, more specifically, the problem of determining the magnetic field generated by the bias current, has to be solved first. On the other hand, the frequency dependence results in a nonlinear eigenproblem. Moreover, if material losses are incorporated, a non-Hermitian eigenvalue problem is obtained. To satisfy these challenging requirements, a dedicated solver has been developed in this thesis.

The solver includes two main subcomponents (cf. Fig. 3.1): The first one is a magnetostatic solver supporting nonlinear materials for the computation of the magnetic field generated by the bias current. The knowledge of the field distribution enables then to evaluate the characteristics of the nonlinear material locally in each mesh cell at the specified working point. The second solver component is an eigensolver for the subsequent solution of the nonlinear eigenvalue problem, which is capable of handling non-Hermitian system matrices. The whole solver is implemented in the programming language $\mathrm{C} / \mathrm{C}++$ and is built on PETSc (Portable, Extensible Toolkit for Scientific Computation) [46, 47]. A simplified class-diagram of the solver is provided in the appendix B. Furthermore, the employed numerical method for the solution of the electromagnetic field problems is based on the Finite Integration Technique, which is introduced in section 3.4, using a hexahedral staircase mesh. For modeling the resonator structure as well as meshing and visualization of the simulation results, CST STUDIO SUITE® [48] is used. 


\subsection{Basics principles of distributed computing}

Because of the clear demand of precise calculations, the implementation particularly aims at high performance for parallel computing on distributed memory machines. Such a computer cluster consists of a set of computers (nodes) with own local memory and a network for the data transfer between the nodes. When a node requires data which are stored on a different node, communication messages have to be sent via the network. The de facto standard to serve this purpose is the Message Passing Interface (MPI) [49, 50], which is also the basis for PETSc. MPI is portable and supports both point-to-point and collective communication. Since the data access via the network is comparatively slow, one should aim at a data interchange as low as possible already at the point of selecting the algorithms and distributing the data. In this regard, some basic knowledge of the parallel structure of vectors and matrices is worthwhile. Commonly, vectors and matrices are distributed onto the $N$ nodes as follows:

$$
\begin{aligned}
& \text { vector: } \\
& \left.\begin{array}{rc}
\text { node } 1\{ & \mathbf{v}_{1} \\
\text { node } 2\{ & \mathbf{v}_{2} \\
\vdots & \vdots \\
\text { node } N\{ & \mathbf{v}_{N}
\end{array}\right)
\end{aligned}
$$

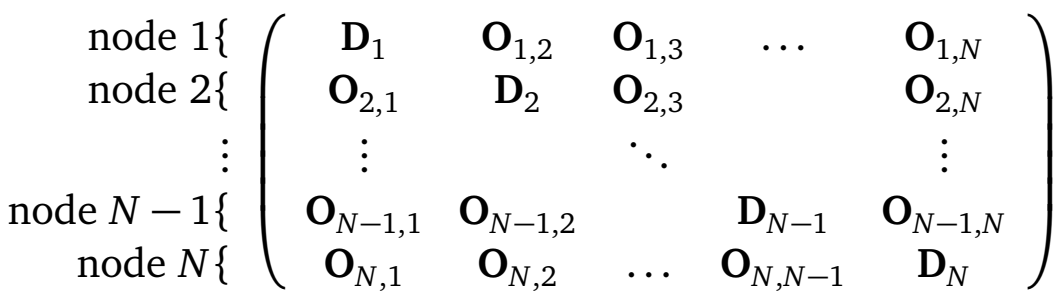

The parts of the vectors $\mathbf{v}_{i}$ as well as the main diagonal and off-diagonal matrix blocks $\mathbf{D}_{i}$ and $\mathbf{O}_{i, j}(i, j=1,2, \cdots, N ; i \neq j)$, respectively, are stored locally in the memory of node $i$. When a matrix-vector product is calculated, it can be split into the products $\mathbf{D}_{i} \mathbf{v}_{i}$ and $\mathbf{O}_{i, j} \mathbf{v}_{j}$. Whereas for the former all required information is locally available on the node, messages have to be communicated between nodes for the latter. Hence, one should take care that there are as few nonzero elements in the off-diagonal matrices $\mathbf{O}_{i, j}$ as possible for performance reasons.

In the implementation in PETSc, all off-diagonal matrix blocks are collected in one single matrix $\mathbf{O}$ on each node [47]. That is to say, a MPI-type matrix is internally built up of two standard, sequential matrices per node. To allow an overlap of communication and computation, the sending of the required vector components is started using a non-blocking MPI function at the beginning of the matrix-vector multiplication. While the messages are passed between the nodes, the matrix-vector product of the main diagonal block is performed locally on each node. After this computation and the communication have been terminated, the off-diagonal part of the matrix-vector product is computed.

\section{Sparse matrix format}

The discretization by the FIT leads to large sparse system matrices with only few nonzero elements. Hence, the storage scheme should take advantage of the sparsity of the matrix. By 
default, PETSc stores sparse matrices in the Compressed Sparse Row (CSR) format [51]. This format stores the $N_{\mathrm{NZ}}$ nonzero elements of a general $m \times n$-matrix in three one-dimensional arrays as follows:

- double (or complex) array $A A$ of length $N_{\mathrm{NZ}}$ : This array stores all nonzero elements sorted in row-major order.

- integer array $J A$ of length $N_{\mathrm{NZ}}$ : This array contains the column indices of each element in $A A$.

- integer array IA of length $m+1$ : Element $i$ of this array holds the index in array $A A$ of the first nonzero element of row $i$. Thus, element 1 of $I A$ is always 1 , whereas the last element is always $N_{\mathrm{NZ}}+1$.

\subsection{The Finite Integration Technique}

The Finite Integration Technique (FIT), a powerful method for the numerical solution of complex electromagnetic field problems, is introduced in this section. For this purpose, the primarydual grid pair is defined in subsection 3.4.1 followed by the allocation of the degrees of freedom (DOFs) in subsection 3.4.2 and the formulation of the Maxwell-Grid-Equations in subsection 3.4.3. Subsequently, the construction of the material matrices is reviewed in subsection 3.4.4. In subsection 3.4.5 the representations of the discretized matrices are discussed for two common ordering schemes as well as for the first time for a fully general one. Electric and magnetic boundary conditions are briefly discussed in subsection 3.4.6. After that, the so-called reduced grid is introduced in subsection 3.4.7, on which, unlike in standard FIT, all for topological reasons vanishing DOFs are completely removed from vectors and matrices. Therein, for the first time an extensive discussion about the pros and cons as well as detailed remarks on an efficient implementation is given. A central part of this thesis is then the extension of the standard FIT to gyrotropic materials in frequency domain in subsection 3.4.8. After that, the curl-curl equation is stated in FIT notation in subsection 3.4.9 followed by some notes on the incorporation of lumped elements in the FIT in subsection 3.4.10. Finally, the solution of nonlinear magnetostatic field problems by means of the so-called $H_{i}$-algorithm is treated in subsection 3.4.11.

Whereas the sections about standard FIT are basically a review of [52], among the main contributions of this thesis are: The concise statement of the ordering scheme for parallel implementation, particularly with the formulation in terms of the most general ordering scheme; the clear classification of the different vector types with regard to their zero pattern, which is a prerequisite for the subsequent discussion of the reduced grid; the extension of the standard FIT to gyrotropic materials in frequency domain.

\subsubsection{Definition of the primary and dual grid}

In the first step, the calculation domain, i.e. the relevant finite three dimensional volume in which Maxwell's equations are to be solved, is defined. For its spatial discretization it is then divided into a grid of a finite number of disjunct and sufficiently small subvolumes, the mesh cells. Though in general any geometry of the grid can be used, this work only deals with grids parameterized by three dimensional Cartesian coordinates. For a Cartesian grid it is convenient 
to enumerate the mesh cells by a set of indices $(i, j, k)$ specifying the cell numbers for the $x$-, $y$ and z-direction, respectively, with $1 \leq i \leq N_{x}, 1 \leq j \leq N_{y}$ and $1 \leq k \leq N_{z}$. Each of the mesh cells is then characterized by a grid point $P(i, j, k)$, a grid volume $V(i, j, k)$, three grid facets $A_{x}(i, j, k), A_{y}(i, j, k)$ and $A_{z}(i, j, k)$ and three grid edges $L_{x}(i, j, k), L_{y}(i, j, k)$ and $L_{z}(i, j, k)$. According to ascending convention, the edge between the nodes $P(i, j, k)$ and $P(i+1, j, k)$ is denoted by $L_{x}(i, j, k)$, whereas the facet $A_{x}(i, j, k)$ is surrounded by the edges $L_{y}(i, j, k)$ and $L_{z}(i, j, k)$. An analogous notation is applicable to the other directions. The orientation of the edges and facets points into the direction of higher indices.

The grid defined before is referred to as the primary grid. Additionally, a second grid, the so-called dual grid, is introduced. It is constructed such a way that each primary cell contains exactly one dual grid point and vice versa. All quantities of the dual grid are marked with the symbol $\sim$ : dual points $\widetilde{P}$, dual edges $\widetilde{L}$, dual facets $\widetilde{A}$ and dual volumes $\widetilde{V}$. In the common case of a dual orthogonal grid, the dual edges are parallel to the ones of the corresponding coordinate direction of the primary grid. Moreover, each dual edge intersects with a primary facet in the center of this facet both having the same indices. This results in a descending indexing for the dual grid. For instance, the dual edge $\widetilde{L}(i, j, k)$ is located between the dual nodes $\widetilde{P}(i-1, j, k)$ and $\widetilde{P}(i, j, k)$. Note the existence of degenerated dual grid elements at the edge of the grid, as the calculation domain is spanned by the primary grid.

\subsubsection{Grid allocation of degrees of freedom}

The DOFs relevant for the formulation of the Maxwell-Grid-Equations are allocated at primary / dual edges and facets as follows:

The electric voltages defined by

$$
\widehat{e}_{a}(i, j, k)=\int_{L_{a}(i, j, k)} \vec{E} \cdot \mathrm{d} \vec{a} \quad(a=x, y, z)
$$

are associated with the primary edges $L_{a}(i, j, k)$. Both the electric fluxes

$$
\widehat{\widehat{d}}_{a}(i, j, k)=\iint_{\widetilde{A}_{a}(i, j, k)} \vec{D} \cdot \mathrm{d} \vec{A} \quad(a=x, y, z)
$$

and the currents

$$
\widehat{\bar{j}}_{a}(i, j, k)=\iint_{\widetilde{A}_{a}(i, j, k)} \vec{J} \cdot \mathrm{d} \vec{A} \quad(a=x, y, z)
$$

are allocated at the dual facets $\widetilde{A}_{a}(i, j, k)$. Moreover, the magnetic fluxes given by

$$
\widehat{\widehat{b}}_{a}(i, j, k)=\iint_{A_{a}(i, j, k)} \vec{B} \cdot \mathrm{d} \vec{A} \quad(a=x, y, z)
$$


are found at the primary facets $A_{a}(i, j, k)$, whereas the magnetic voltages

$$
\widehat{h}_{a}(i, j, k)=\int_{\widetilde{L}_{a}(i, j, k)} \vec{H} \cdot \mathrm{d} \vec{a} \quad(a=x, y, z)
$$

are associated with the dual edges $\widetilde{L}_{a}(i, j, k)$. Finally, the electric charges inside a dual mesh cell are defined as

$$
q(i, j, k)=\iint_{\widetilde{V}(i, j, k)} \rho \mathrm{d} V
$$

pseudo magnetic charges ${ }^{1}$ inside a primary cell as

$$
q_{\mathrm{m}}(i, j, k)=\iint_{V(i, j, k)} \int_{\mathrm{m}} \mathrm{d} V .
$$

To sum up, from the topological point of view the DOFs can be classified into six different types: DOFs allocated on primary edges, primary faces and primary nodes on the one hand, the ones allocated on dual edges, dual faces and dual nodes on the other hand.

\subsubsection{Maxwell-Grid-Equations}

Employing the definitions of the previous section, Maxwell's Equations (cf. section 2.1) can be rewritten as a set of matrix equations. The procedure is illustrated for Faraday's law for nonmoving geometries (2.4). Taking the area integration $A$ therein as the primary facet $A_{z}(i, j, k)$ and taking into account equations (3.7) and (3.10) as well as the orientation of $\widehat{e}_{a}$ and $\widehat{\vec{b}}_{a}$, Faraday's law takes the form

$$
\widehat{e}_{x}(i, j, k)+\widehat{e}_{y}(i+1, j, k)-\widehat{e}_{x}(i, j+1, k)-\widehat{e}_{y}(i, j, k)=-\frac{\mathrm{d}}{\mathrm{d} t} \widehat{\bar{b}}_{z}(i, j, k)
$$

Analogously, equations for all other primary facets are obtained, which are arranged as one matrix equation

$$
\mathbf{C} \widehat{\mathbf{e}}=-\frac{\mathrm{d}}{\mathrm{d} t} \widehat{\widehat{\mathbf{b}}}
$$

Herein, vectors $\widehat{\mathbf{e}}$ and $\widehat{\widehat{\mathbf{b}}}$ collect all electric voltages and magnetic fluxes, respectively. The topological matrix $\mathbf{C}$ ensures that the components of $\widehat{\mathbf{e}}$ are added with the right signs. It is usually referred to as the discrete curl operator at the primary grid though it actually performs the computation of the contour-integral on primary facets. Note that the explicit form of $\mathbf{C}$ depends on

$\overline{1}$ Though according to current conception magnetic charges do not exist, sometimes it is mathematically advantageous to introduce pseudo magnetic charges, see, e.g., the $H_{i}$-algorithm (cf. subsection 3.4.11.2). 
the ordering of the elements in the vectors $\widehat{\mathbf{e}}$ and $\widehat{\overline{\mathbf{b}}}$. Though the arrangement could in principle be chosen arbitrarily, those which allow an easy and efficient implementation should be preferred. Two different frequently used ordering schemes together with the explicit representation of the matrix $\mathrm{C}$ therein are discussed in subsection 3.4.5.

In an analogous manner the remaining Maxwell-Grid-Equations are obtained:

$$
\begin{aligned}
& \widetilde{\mathbf{C}} \widehat{\mathbf{h}}=\frac{\mathrm{d}}{\mathrm{d} t} \widehat{\widehat{\mathbf{d}}}+\widehat{\mathbf{j}}, \\
& \widetilde{\mathbf{S}} \widehat{\widehat{\mathbf{d}}}=\mathbf{q}, \\
& \widehat{\mathbf{S}} \overline{\mathbf{b}}=0 .
\end{aligned}
$$

Herein, the matrix $\mathbf{S}$ yields the integral of a vector field (allocated on primary facets) over the surface of a primary cell and is usually considered as the discrete counterpart of the divergence operator. $\widetilde{\mathbf{C}}$ and $\widetilde{\mathbf{S}}$ may be interpreted as the discrete curl and divergence operator at the dual grid, respectively. Again, the explicit form of these topological matrices depends on the ordering scheme of the elements in the vectors (cf. subsection 3.4.5). The electric fluxes, currents and magnetic voltages are collected in the vectors $\widehat{\mathbf{d}}, \widehat{\mathbf{j}}$ and $\widehat{\mathbf{h}}$, respectively. Vector $\mathbf{q}$ represents the electric charges inside the dual cells. One important property of the four Maxwell-Grid-Equations (3.15) to (3.18) is that they are exact. Approximations need, however, to be introduced for the discretization of constitutive equations, which will be discussed in the next section.

\subsubsection{Construction of the material matrices}

Besides Maxwell's Equations the constitutive equations need to be discretized. For isotropic linear materials the constitutive equations read $\left(v:=\mu^{-1}\right)$ :

$$
\begin{aligned}
\vec{H} & =v \vec{B} \\
\vec{D} & =\varepsilon \vec{E}
\end{aligned}
$$

Following the notation introduced before, the discrete counterparts take the form (for component $a=x, y, z)$ :

$$
\begin{aligned}
& \widehat{h}_{a}=\left(\mathbf{M}_{v}\right)_{a} \widehat{\widehat{b}}_{a} \\
& \widehat{\widehat{d}}_{a}=\left(\mathbf{M}_{\varepsilon}\right)_{a} \widehat{e}_{a}
\end{aligned}
$$

The determination of the components of the discrete inverse permeability matrix $\mathbf{M}_{v}$ and the discrete permittivity matrix $\mathbf{M}_{\varepsilon}$ as the ratio of the approximations of the corresponding integrated quantities is discussed below.

\section{Permeability}

For the construction of the inverse permeability matrix consider figure 3.2(a), which shows the intersection of the magnetic flux $\widehat{\vec{b}}_{a}(i, j, k)$ with the primary facet $A_{a}(i, j, k)$. It is assumed that the left cell is homogeneously filled with a material with permeability $\mu_{1}$ whereas the right 


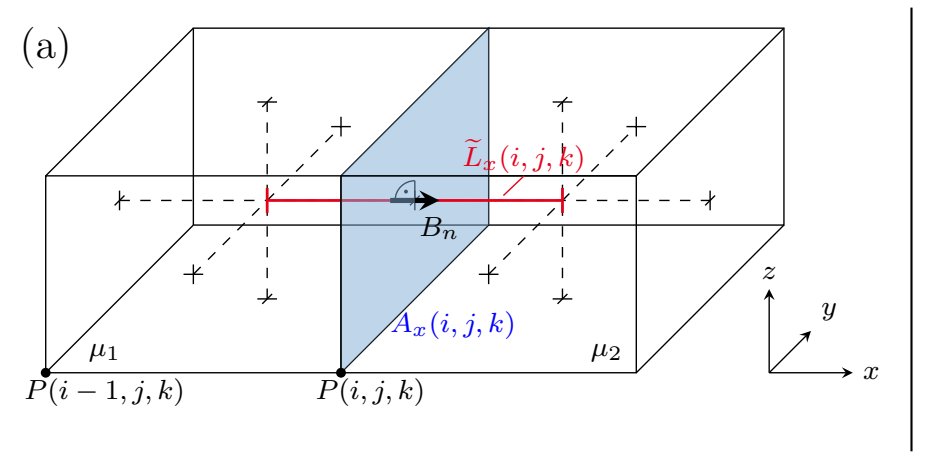

(b)

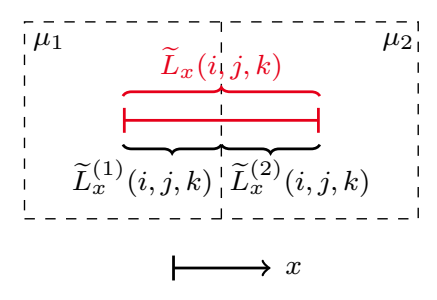

Figure 3.2.: (a) Intersection of the dual edge $\widetilde{L}_{x}(i, j, k)$ with the primary facet $A_{x}(i, j, k)$. For the construction of the inverse permeability matrix, the inverse permeability values $v_{i}=\mu_{i}^{-1}(i=1,2)$ are averaged along the dual edge. (b) Notation for the length of line segments of dual edges. If the superscript is present, only the partial length within the primary cell with the index given in the superscript is considered.

cell has permeability $\mu_{2}$. Since the component of the magnetic flux normal to the primary facet $B_{n}$ is continuous, $\widehat{\vec{b}}_{a}(i, j, j, k)$ can be well approximated with

$$
\widehat{\widehat{b}}_{a}(i, j, k)=\iint_{A_{a}(i, j, k)} \vec{B} \cdot \mathrm{d} \vec{A} \approx B_{n} A_{a}(i, j, k) .
$$

The magnetic voltage $\widehat{h}_{a}(i, j, k)$ along the dual edge $\widetilde{L}_{a}(i, j, k)$ is not continuous at the point of intersection in general. Hence, $\widehat{h}_{a}(i, j, k)$ can be expressed approximately as

$$
\widehat{h}_{a}(i, j, k)=\int_{\widetilde{L}_{a}(i, j, k)} \vec{H} \cdot \mathrm{d} \vec{a}=\int_{\widetilde{L}_{a}^{(1)}(i, j, k)} \frac{B_{n}}{\mu_{1}} \mathrm{~d} s+\int_{\widetilde{L}_{a}^{(2)}(i, j, k)} \frac{B_{n}}{\mu_{2}} \mathrm{~d} s \approx B_{n} \bar{v}_{a}(i, j, k) \widetilde{L}_{a}(i, j, k),
$$

with the averaged inverse permeability $\left(v=\mu^{-1}\right)$

$$
\bar{v}_{a}(i, j, k)=\frac{v_{1} \widetilde{L}_{a}^{(1)}(i, j, k)+v_{2} \widetilde{L}_{a}^{(2)}(i, j, k)}{\widetilde{L}_{a}(i, j, k)} .
$$

Herein, $\widetilde{L}_{a}^{(1)}(i, j, k)$ and $\widetilde{L}_{a}^{(2)}(i, j, k)$ are the length of the dual edge only of the part inside the primary cell 1 and 2, respectively (see also figure 3.2(b)). Comparing the defining equation (3.21) with equations (3.23) and (3.24), yields for the component of the inverse permeability matrix

$$
\left(\mathbf{M}_{v}\right)_{a}(i, j, k)=\frac{\bar{v}_{a}(i, j, k) \widetilde{L}_{a}(i, j, k)}{A_{a}(i, j, k)} .
$$

\section{Permittivity}

The tangential component of the electric field $E_{t}$ is continuous at the interface between two media. Hence,

$$
\widehat{e}_{a}(i, j, k)=\int_{L_{a}(i, j, k)} \vec{E} \cdot \mathrm{d} \vec{a} \approx E_{t} L_{a}(i, j, k)
$$


(a)

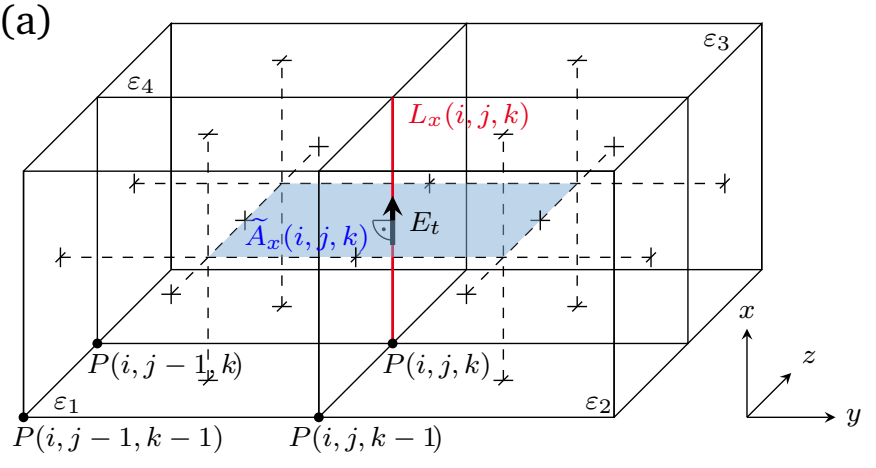

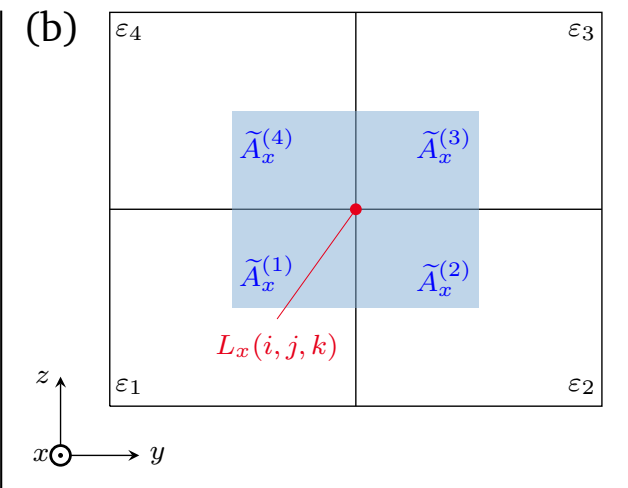

Figure 3.3.: (a) Intersection of the primary edge $L_{x}(i, j, k)$ with the dual facet $\widetilde{A}_{x}(i, j, k)$. For the construction of the permittivity matrix, the permittivity values $\varepsilon_{1}, \varepsilon_{2}, \varepsilon_{3}$ and $\varepsilon_{4}$ are averaged on the dual facet. (b) Notation for the area of partial dual facets. If the superscript is present, only the partial area within the primary cell with the index given in the superscript is considered.

is a good approximation for the electric voltage $\widehat{e}_{a}(i, j, k)$ on the primary edge $L_{a}(i, j, k)$. However, the component of the electric fluxes tangential to the interface $\widehat{\widehat{d}}_{a}(i, j, k)$ is discontinuous, which is taken into account by dividing the integral into four parts (cf. figure 3.3):

$$
\widehat{\vec{d}}_{a}(i, j, k)=\iint_{\widetilde{A}_{a}(i, j, k)} \vec{D} \cdot \mathrm{d} \vec{A}=\sum_{n=1}^{4} \iint_{\widetilde{A}_{a}^{(n)}} E_{(i, j, k)} \mathrm{d} A \approx E_{t} \bar{\varepsilon}_{a}(i, j, k) \widetilde{A}_{a}(i, j, k) .
$$

The averaged permittivity herein is given by

$$
\bar{\varepsilon}_{a}(i, j, k)=\sum_{n=1}^{4} \frac{\varepsilon_{n} \widetilde{A}_{a}^{(n)}(i, j, k)}{\widetilde{A}_{a}(i, j, k)}
$$

where $\widetilde{A}_{a}^{(n)}(i, j, k)$ is the partial area of the dual facet $\widetilde{A}_{a}(i, j, k)$ inside the primary cell $n$ (see also Fig. 3.3(b)). The component of the permittivity matrix is obtained by comparing the defining equation (3.22) with equations (3.27) and (3.28) as

$$
\left(\mathbf{M}_{\varepsilon}\right)_{a}(i, j, k)=\frac{\bar{\varepsilon}_{a}(i, j, k) \tilde{A}_{a}(i, j, k)}{L_{a}(i, j, k)} .
$$

The constitutive relations can also be expressed as matrix equations. To this end, all the lengths of the primary and dual edges as well as the areas of the primary and dual facets are collected in the diagonal matrices $\mathbf{D}_{L}, \widetilde{\mathbf{D}}_{L}$ and $\mathbf{D}_{A}, \widetilde{\mathbf{D}}_{A}$, respectively. Analogously, the diagonal matrices $\mathbf{D}_{v}$ and $\mathbf{D}_{\varepsilon}$ gather the averaged values of the inverse permeability and the permittivity, respectively. Hence, equations (3.26) and (3.30) can be expressed as

$$
\begin{aligned}
& \mathbf{M}_{v}=\widetilde{\mathbf{D}}_{L} \mathbf{D}_{v} \mathbf{D}_{A}^{-1}, \\
& \mathbf{M}_{\varepsilon}=\widetilde{\mathbf{D}}_{A} \mathbf{D}_{\varepsilon} \mathbf{D}_{L}^{-1} .
\end{aligned}
$$

Note that the above material matrices $\mathbf{M}_{v}$ and $\mathbf{M}_{\varepsilon}$ are consequently diagonal in the standard FIT. Of course, the ordering of the parameters in the matrices introduced above has to match the 
ordering chosen for the vectors, which collect the DOFs (cf. section 3.4.3). With the definitions (3.31) and (3.32), the component-wise constitutive relations (3.21) and (3.22) can thus be written in matrix-vector notation as

$$
\begin{aligned}
& \widehat{\mathbf{h}}=\mathbf{M}_{\nu} \widehat{\widehat{\mathbf{b}}}, \\
& \widehat{\widehat{\mathbf{d}}}=\mathbf{M}_{\varepsilon} \widehat{\mathbf{e}} .
\end{aligned}
$$

\subsubsection{Discretized matrices}

As mentioned in section 3.4.3, the DOFs are collected in vectors, which allows to write the Maxwell-Grid-Equations conveniently as a set of matrix equations. The vector components can be arranged arbitrarily as long as this is done consistently in all the involved vectors and matrices. In other words, having set the ordering of the DOFs, the structure of all operators and in particular of the topological matrices is fully fixed. When determining the ordering scheme, aspects such as a convenient notation and the possibility of an easy and efficient implementation should be considered. The arrangement which is best suited may depend on the type and size of the problem. In this section, the two most commonly used ordering schemes for a Cartesian grid are introduced and the structure of the topological matrices therein is presented. The first one was originally presented by Weiland [53] and is the standard in current implementations. As will be seen in subsection 3.4.5.1, the main advantage of this scheme is that the topological matrices can be composed by only two independent types of two-banded submatrices, which enormously facilitates both theoretical calculations and implementation. In contrast, the second ordering scheme (cf. subsection 3.4.5.2) is advantageous for parallel computing on machines with distributed memory. After that, a discussion of the most general form of the topological matrices for any arbitrary ordering scheme follows in subsection 3.4.5.3. As one of the achievements of the thesis at hand, this also allows to manifestly reveal the same topological structure of the two before-mentioned ordering schemes. Finally, this subsection is completed by the presentation of some important properties of the topological matrices in subsection 3.4.5.4.

\subsubsection{Ordering 1 (standard FIT)}

The following subsection reviews the discussion of [52]. In the ordering scheme of the standard FIT, the vectors are divided into three subvectors: The first one only contains the $x$-components, the second one all the $y$-components and finally the third one the $z$-components. Inside each subvector the components are arranged as follows: One starts with the component with lowest 
cell indices ( $i=1, j=1, k=1$ ) and proceeds firstly in $x$-direction, then in $y$-direction and finally in $z$-direction. In summary, the components of a generic vector $\mathbf{v}$ are arranged as:

$$
\left.\mathbf{v}_{a}:=\left(\begin{array}{c}
v_{a}(1,1,1) \\
\ldots \\
v_{a}\left(N_{x}, 1,1\right) \\
v_{a}(1,2,1) \\
\cdots \\
v_{a}\left(N_{x}, 2,1\right) \\
\ldots \\
v_{a}\left(N_{x}, N_{y}, 1\right) \\
v_{a}(1,1,2) \\
\cdots \\
v_{a}\left(N_{x}, N_{y}, N_{z}\right)
\end{array}\right) \text { (for } a=x, y, z\right) ; \quad \mathbf{v}:=\left(\begin{array}{c}
\mathbf{v}_{x} \\
\mathbf{v}_{y} \\
\mathbf{v}_{z}
\end{array}\right) .
$$

The total number of vector components thus is $3 N_{x} N_{y} N_{z}=: 3 N_{\text {cells }}$, where $N_{\text {cells }}$ is the number of all (primary) grid cells. Having fixed the arrangement of the components, also the structure of the topological matrices is determined, which is derived in the following. For this purpose, it is useful to formulate a relation between the position $n$ of a vector component within a subvector on the one hand and the cell indices $i, j$ and $k$ on the other hand, which reads

$$
\begin{aligned}
n & =(i-1)+(j-1) N_{x}+(k-1) N_{x} N_{y}+1 \\
& =(i-1) M_{x}^{\prime}+(j-1) M_{y}^{\prime}+(k-1) M_{z}^{\prime}+1 .
\end{aligned}
$$

In the last transformation in the equation above the definitions

$$
\begin{array}{ll}
M_{x}^{\prime}:=1, & \text { (for ordering 1) } \\
M_{y}^{\prime}:=N_{x}, & \text { (for ordering 1) } \\
M_{z}^{\prime}:=N_{y} N_{z} & \text { (for ordering 1) }
\end{array}
$$

are introduced. The quantity $M_{a}^{\prime}(a=x, y, z)$ can be interpreted as the number by which the position $n$ increases if one proceeds one cell in direction $a$.

For a start, the structure of the matrix $\mathrm{C}$ is considered for ordering 1 . As pointed out in section 3.4.3, each row of the matrix $\mathbf{C}$ extracts the vector components that contribute to the contourintegral on a primary facet with the correct sign. In this ordering scheme, the first third of the rows corresponds to primary facets with a normal vector in $x$-direction. The contour-integral of the generic vector $\mathbf{v}$ on the $n$-th facet ( $x$-direction) takes the form

$$
\begin{aligned}
\oint_{\partial A_{x}(n)} \vec{v} \cdot \mathrm{d} \vec{s} & =v_{y}(i, j, k)+v_{z}(i, j+1, k)-v_{y}(i, j, k+1)-v_{z}(i, j, k) \\
& =v_{y}(n)+v_{z}\left(n+M_{y}^{\prime}\right)-v_{y}\left(n+M_{z}^{\prime}\right)-v_{z}(n) .
\end{aligned}
$$


Similar expressions are obtained for facets in $y$ - and $z$-direction. Since all these contourintegrals involve expressions of the form $\pm v_{a}(n) \mp v_{a}\left(n+M_{b}^{\prime}\right),(a, b=x, y, z ; a \neq b)$, for ease of notation the $N_{\text {cells }} \times N_{\text {cells }}$ submatrices

$$
\begin{aligned}
& {\left[\mathbf{P}_{x}\right]_{p, q}=\left\{\begin{array}{rl}
-1 & q=p \\
+1 & q=p+M_{x}^{\prime} \\
0 & \text { else }
\end{array}\right\},} \\
& {\left[\mathbf{P}_{y}\right]_{p, q}=\left\{\begin{array}{rl}
-1 & q=p \\
+1 & q=p+M_{y}^{\prime} \\
0 & \text { else }
\end{array}\right\},} \\
& {\left[\mathbf{P}_{z}\right]_{p, q}=\left\{\begin{array}{rl}
-1 & q=p \\
+1 & q=p+M_{z}^{\prime} \\
0 & \text { else }
\end{array}\right\}}
\end{aligned}
$$

are introduced, which correspond to the discretized differential operators $\partial / \partial x, \partial / \partial y$ and $\partial / \partial z$. The structure of the matrix $\mathbf{C}$ with dimension $3 N_{\text {cells }} \times 3 N_{\text {cells }}$ can then be composed as

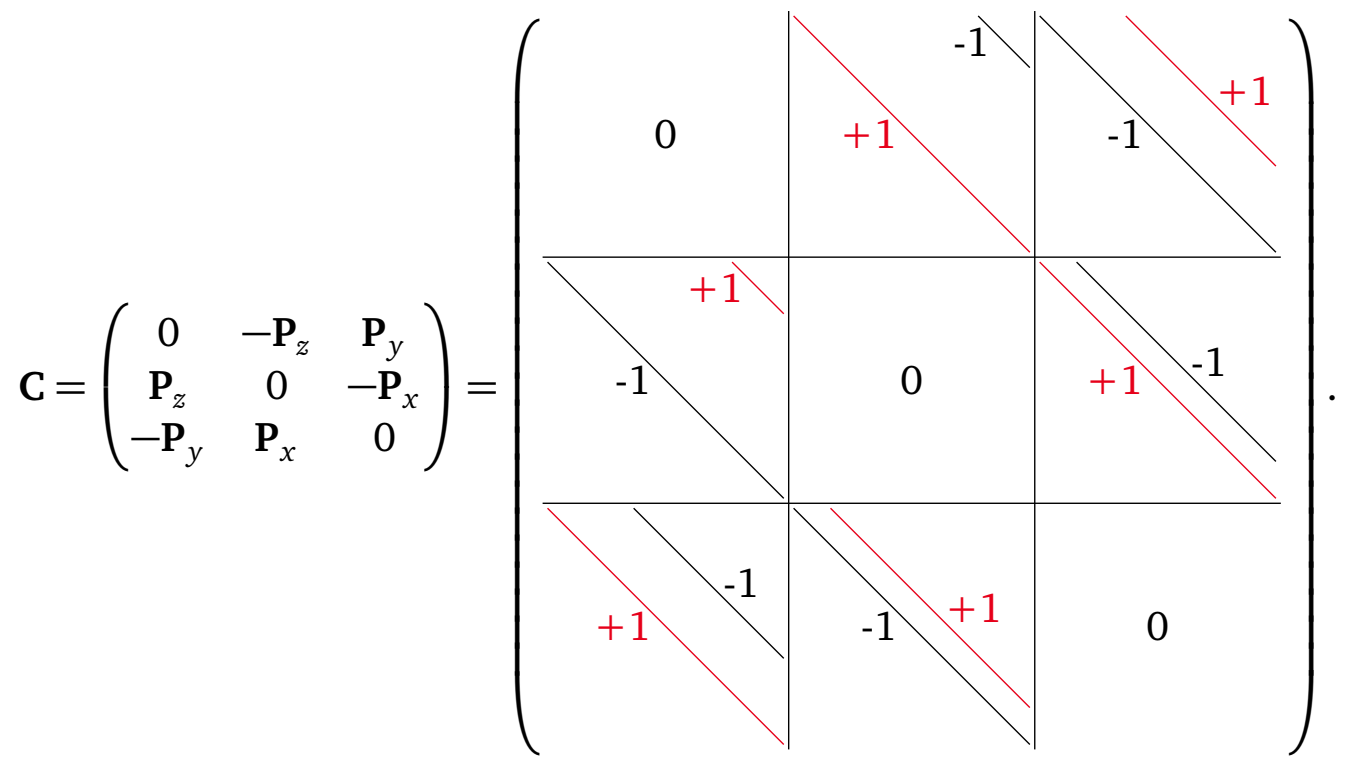

Secondly, the structure of the matrix $\mathbf{S}$ is analyzed. Its $n$-th row measures the magnitude of a vector field's source at the $n$-th primary cell by summing up the relevant vector components with the correct sign as

$$
\begin{array}{r}
\oint_{\partial V(n)} \vec{v} \cdot \mathrm{d} \vec{A}=v_{x}(i, j, k)+v_{y}(i, j, k)+v_{z}(i, j, k)-v_{x}(i+1, j, k)-v_{y}(i, j+1, k)-v_{z}(i, j, k+1)= \\
=v_{x}(n)+v_{y}(n)+v_{z}(n)-v_{x}\left(n+M_{x}^{\prime}\right)-v_{y}\left(n+M_{y}^{\prime}\right)-v_{z}\left(n+M_{z}^{\prime}\right) . \quad(3.41)
\end{array}
$$

Again, the integrals can be conveniently written in terms of the $\mathbf{P}$-submatrices as

$$
\mathbf{S}=\left(\begin{array}{lll}
\mathbf{P}_{x} & \mathbf{P}_{y} & \mathbf{P}_{z}
\end{array}\right)=(-1
$$


In a similar manner, the structure of the corresponding matrices on the dual grid is obtained. However, due to the descending indexing convention (cf. section 3.4.1) additional $N_{\text {cells }} \times N_{\text {cells }}{ }^{-}$ submatrices $\widetilde{\mathbf{P}}$

$$
\begin{gathered}
{\left[\widetilde{\mathbf{P}}_{x}\right]_{p, q}=\left\{\begin{array}{rl}
+1 & q=p \\
-1 & q=p-M_{x}^{\prime} \\
0 & \text { else }
\end{array}\right\},} \\
{\left[\widetilde{\mathbf{P}}_{y}\right]_{p, q}=\left\{\begin{array}{rl}
+1 & q=p \\
-1 & q=p-M_{y}^{\prime} \\
0 & \text { else }
\end{array}\right\},} \\
{\left[\widetilde{\mathbf{P}}_{z}\right]_{p, q}=\left\{\begin{array}{rl}
+1 & q=p \\
-1 & q=p-M_{z}^{\prime} \\
0 & \text { else }
\end{array}\right\},}
\end{gathered}
$$

corresponding to the discretized differential operators on the dual grid, are required. The matrices $\widetilde{\mathbf{C}}$ and $\widetilde{\mathbf{S}}$ then take the form

$$
\widetilde{\mathbf{C}}=\left(\begin{array}{ccc}
0 & -\widetilde{\mathbf{P}}_{z} & \widetilde{\mathbf{P}}_{y} \\
\widetilde{\mathbf{P}}_{z} & 0 & -\widetilde{\mathbf{P}}_{x} \\
-\widetilde{\mathbf{P}}_{y} & \widetilde{\mathbf{P}}_{x} & 0
\end{array}\right)=\left(\begin{array}{c}
0 \\
\end{array}\right.
$$

and

$$
\widetilde{\mathbf{S}}=\left(\begin{array}{lll}
\widetilde{\mathbf{P}}_{x} & \widetilde{\mathbf{P}}_{y} & \widetilde{\mathbf{P}}_{z}
\end{array}\right)=(-1)+1
$$

respectively. For completeness, also the structure of the discretized matrix versions of the gradient operator on the primary and dual grid, denoted by $\mathbf{G}$ and $\widetilde{\mathbf{G}}$, respectively, is considered. Once again, $\mathbf{G}$ can be conveniently expressed in terms of the submatrices $\mathbf{P}$,

$$
\mathbf{G}=\left(\begin{array}{l}
\mathbf{P}_{x} \\
\mathbf{P}_{y} \\
\mathbf{P}_{z}
\end{array}\right),
$$



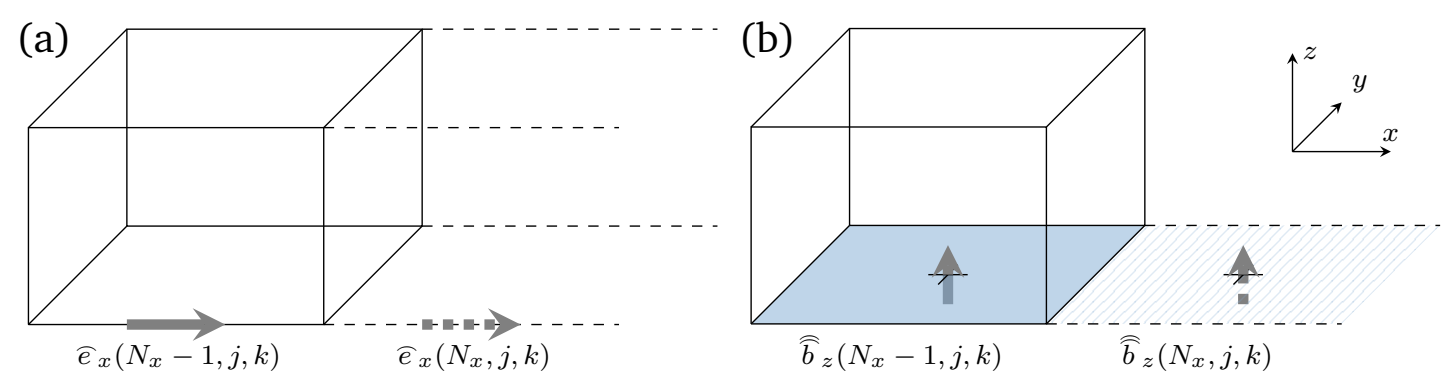

Figure 3.4.: Examples for primary edges (a) and primary facets (b) outside the computation domain. Solid lines and facets are located inside, dashed lines and hatched facets outside the computation domain.

whereas $\widetilde{\mathbf{G}}$ is given by

$$
\widetilde{\mathbf{G}}=\left(\begin{array}{l}
\widetilde{\mathbf{P}}_{x} \\
\widetilde{\mathbf{P}}_{y} \\
\widetilde{\mathbf{P}}_{z}
\end{array}\right) .
$$

It is important to mention that the vector, when introduced as in definition (3.35), contains components which are allocated on elements outside the actual computation domain, more precisely, at its upper edge towards large indices. For quantities allocated on primary edges or dual facets, such as for $\widehat{\mathbf{e}}$ and $\widehat{\mathbf{d}}$, these are the components $v_{x}\left(N_{x}, j, k\right), v_{y}\left(i, N_{y}, k\right)$ and $v_{z}\left(i, j, N_{z}\right)$. In contrast, for vectors gathering quantities allocated on dual edges or primary facets, such as $\widehat{\mathbf{h}}$ and $\widehat{\mathrm{b}}$, these components are $v_{x}\left(i, N_{y}, k\right), v_{x}\left(i, j, N_{z}\right), v_{y}\left(N_{x}, i, k\right), v_{y}\left(i, j, N_{z}\right), v_{z}\left(N_{x}, j, k\right)$ and $v_{z}\left(i, N_{y}, k\right)$. This is also illustrated in figure 3.4. Instead of removing these components completely from the vector, in the standard FIT they are just zeroed in order not to damage the band structure of the matrices. For a detailed discussion whether or not it is beneficial in doing so is referred to subsection 3.4.7.2. Note, however, that in the preceding discussion of the structure of the topological matrices neither the components outside the computation domain nor potential vanishing components due to boundary conditions (cf. subsection 3.4.6) are taken into account. This is supplemented in subsection 3.4.7.1.

The main advantage of ordering scheme 1 is obviously the clear band structure of all topological matrices. This allows both an easy and efficient implementation. Moreover, the memory requirements for the storage of the matrices are very moderate. Yet, this ordering scheme is not ideal for parallel computing with distributed memory. Looking for example at the structure of the matrix C (cf. Eq. (3.40)), it clearly shows that the principle to have only a minimum number of nonzero elements in the far off-diagonal regions of the matrix (cf. section 3.3) is not respected. Indeed, the submatrices located in the main-diagonal of the matrix are even completely zero. Similar considerations apply for the other topological matrices. Hence, a second ordering scheme to cope with this issue is discussed in the next subsection.

\subsubsection{Ordering 2 (for parallel computing)}

In this ordering scheme, the cells are run through in the direction $x$ firstly, then in the direction $y$, and finally in the direction $z$, just as in the previous scheme. The difference, however, is that 
the $x$-, $y$ - and $z$-components of a vector for each cell are arranged directly one after another. This results in the following assembly:

$$
\begin{aligned}
\mathbf{v}:= & v_{x}(1,1,1), v_{y}(1,1,1), v_{z}(1,1,1), \\
& \cdots \\
& v_{x}\left(N_{x}, 1,1\right), v_{y}\left(N_{x}, 1,1\right), v_{z}\left(N_{x}, 1,1\right), \\
& v_{x}(1,2,1), v_{y}(1,2,1), v_{z}(1,2,1), \\
& \cdots \\
& v_{x}\left(N_{x}, 2,1\right), v_{y}\left(N_{x}, 2,1\right), v_{z}\left(N_{x}, 2,1\right), \\
& \cdots \\
& v_{x}\left(N_{x}, N_{y}, 1\right), v_{y}\left(N_{x}, N_{y}, 1\right), v_{z}\left(N_{x}, N_{y}, 1\right), \\
& v_{x}(1,1,2), v_{y}(1,1,2), v_{z}(1,1,2), \\
& \cdots, \\
& \left.v_{x}\left(N_{x}, N_{y}, N_{z}\right), v_{y}\left(N_{x}, N_{y}, N_{z}\right), v_{z}\left(N_{x}, N_{y}, N_{z}\right)\right)^{T},
\end{aligned}
$$

where $\mathbf{v}$ is a generic vector (allocated either on the primary or dual grid). Consequently, also the structure of all topological matrices has to be adapted. Following a similar approach as for ordering scheme 1 , initially a relation between the position $n$ of a vector component and the cell indices $i, j$ and $k$ is useful, which takes the form

$$
\begin{aligned}
n & =(i-1) 3+(j-1) 3 N_{x}+(k-1) 3 N_{x} N_{y}+1 \\
& =(i-1) M_{x}^{\prime \prime}+(j-1) M_{y}^{\prime \prime}+(k-1) M_{z}^{\prime \prime}+1 .
\end{aligned}
$$

The quantities $M_{a}^{\prime \prime}(a=x, y, z)$, given by

$$
\begin{array}{ll}
M_{x}^{\prime \prime}:=3, & \text { (for ordering 2) } \\
M_{y}^{\prime \prime}:=3 N_{x}, & \text { (for ordering 2) } \\
M_{z}^{\prime \prime}:=3 N_{y} N_{z}, & \text { (for ordering 2) }
\end{array}
$$

are the numbers by which the position $n$ increases if one proceeds one cell in direction $a$. The matrix $\mathrm{C}$ now takes the form $\left(1 \leq p, q \leq 3 N_{\text {cells }}\right)$ :

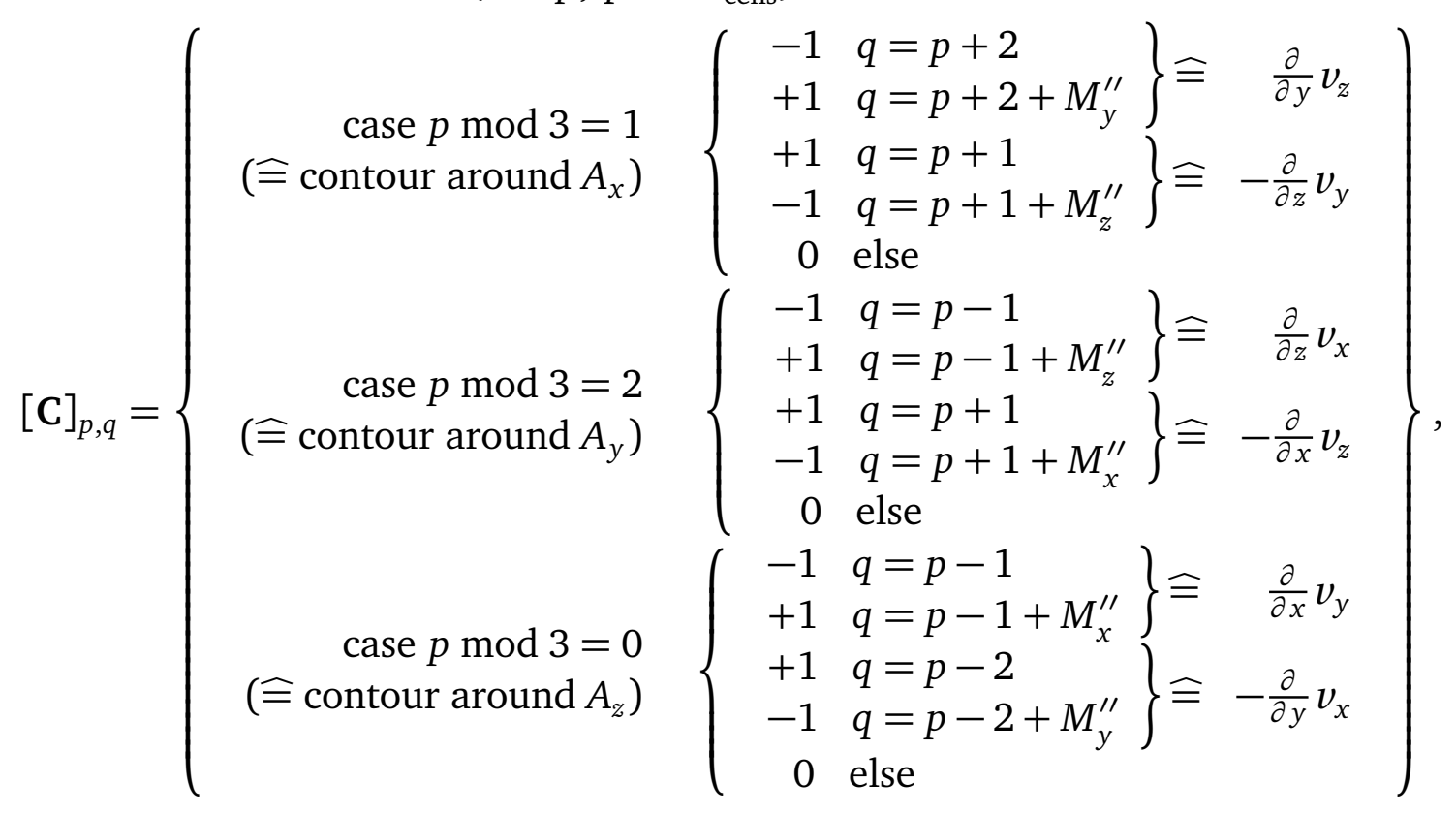




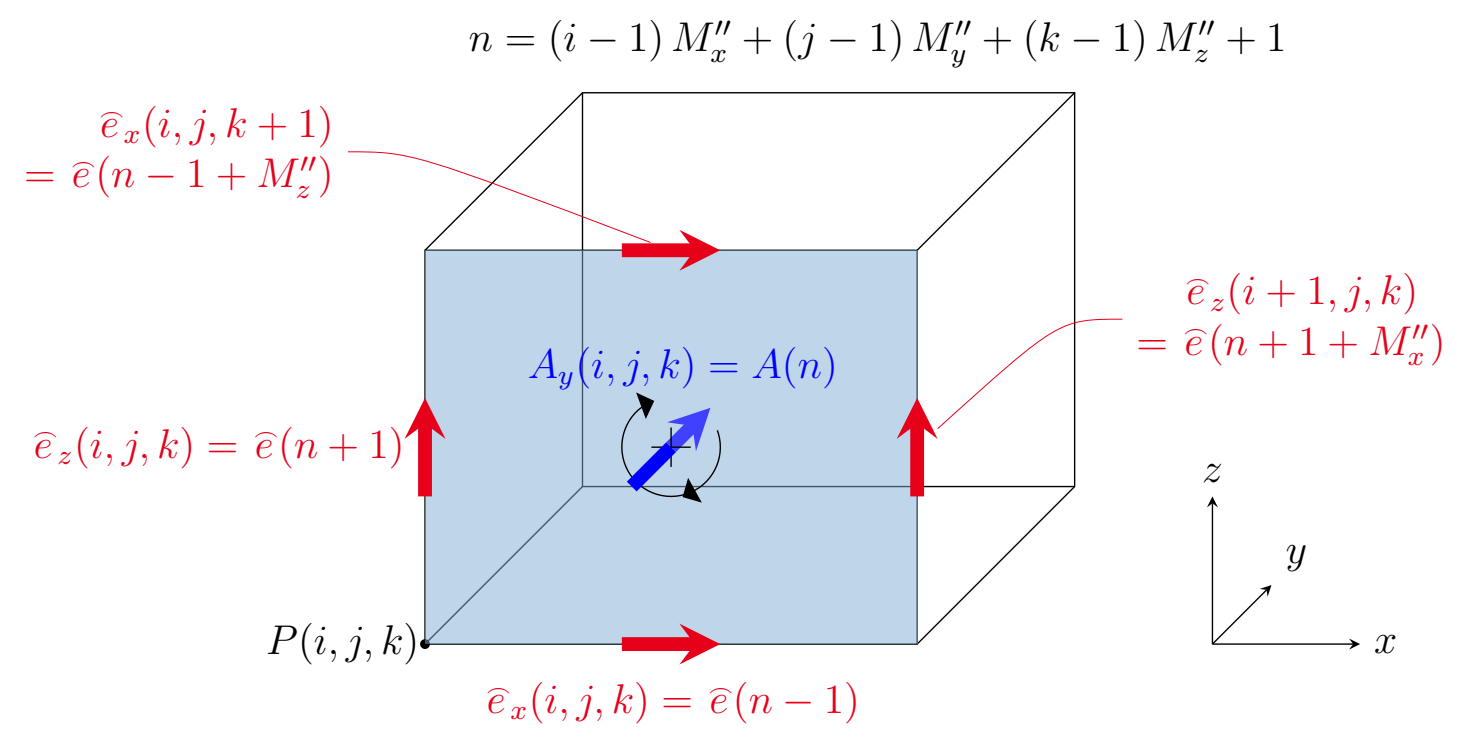

Figure 3.5.: To the structure of the matrix $\mathbf{C}$ in ordering scheme 2. As an example, the contour integral around a primary area with orientation in $y$-direction is illustrated (i.e. for case $p \bmod 3=2$ in Eq. (3.51)).

wherein $p$ mod 3 gives the remainder on a division of $p$ by 3 . For a better understanding of its structure, figure 3.5 shows the contour integral around a primary area with orientation in $y$-direction, which corresponds to case $p \bmod 3=2$. As indicated on the right hand side of equation (3.51), the analogy with the analytical curl-operator is still evident also for this ordering scheme. Yet, it does not show up in a block structure, which can be represented by $\left(N_{\text {cells }} \times N_{\text {cells }}\right)$-submatrices (cf. Eq. (3.40)), but in the sequence of three successive rows. Analogously, the expression for the curl matrix on the dual grid is derived as $\left(1 \leq p, q \leq 3 N_{\text {cells }}\right.$ )

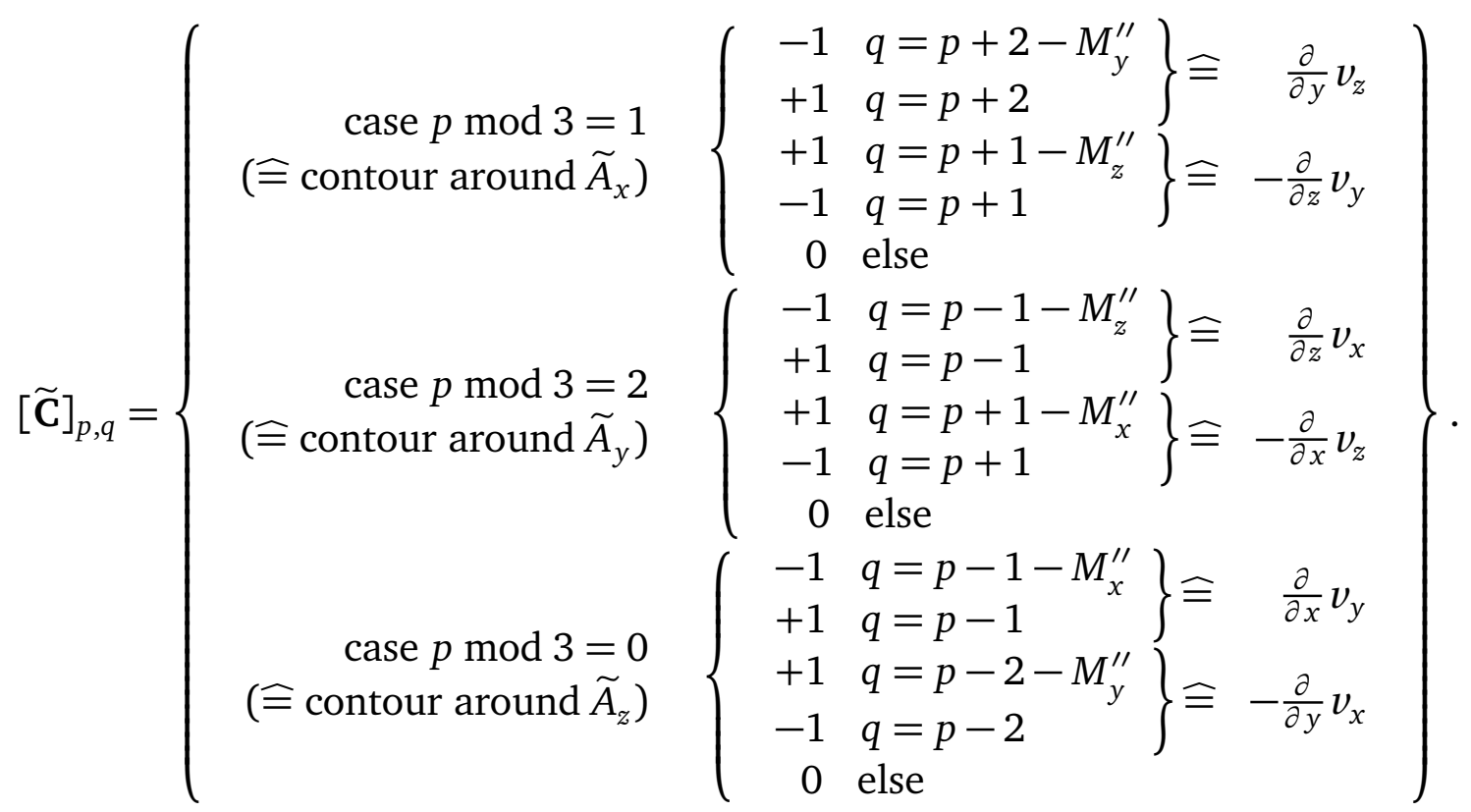


The matrix $\mathbf{S}$ now is given by $\left(1 \leq p \leq N_{\text {cells }} ; 1 \leq q \leq 3 N_{\text {cells }}\right)$

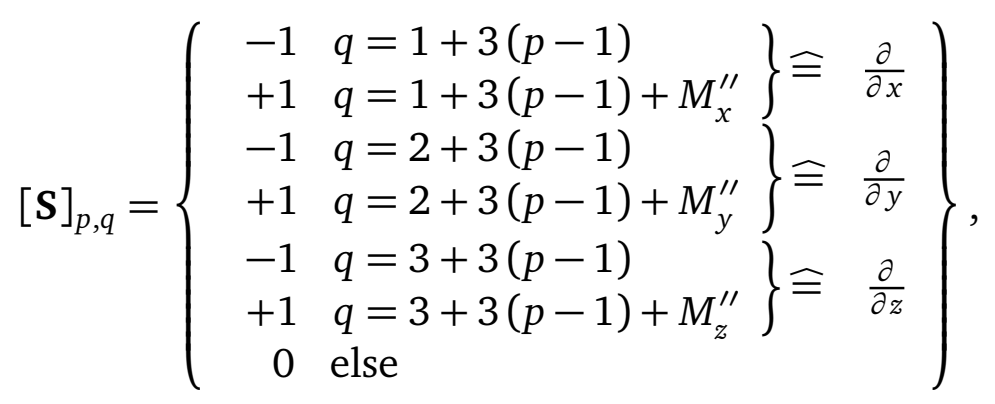

the correspondent on the dual grid by ( $1 \leq p \leq N_{\text {cells }} ; 1 \leq q \leq 3 N_{\text {cells }}$ )

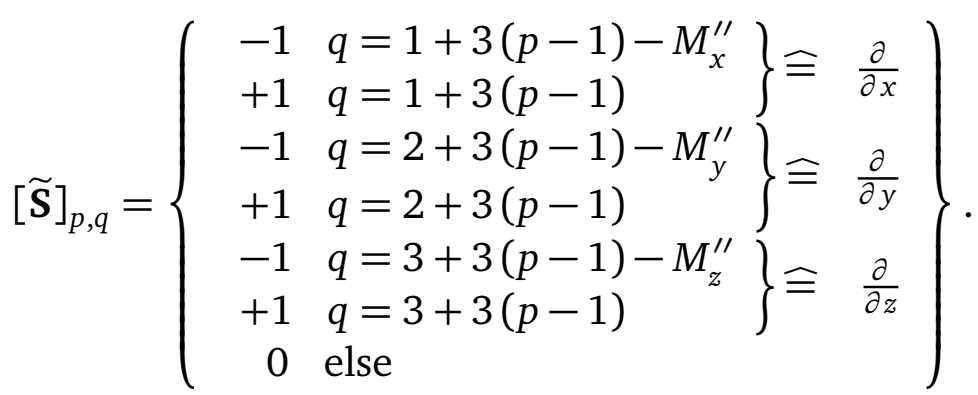

Finally, the gradient matrices take the form $\left(1 \leq p \leq 3 N_{\text {cells }} ; 1 \leq q \leq N_{\text {cells }}\right)$

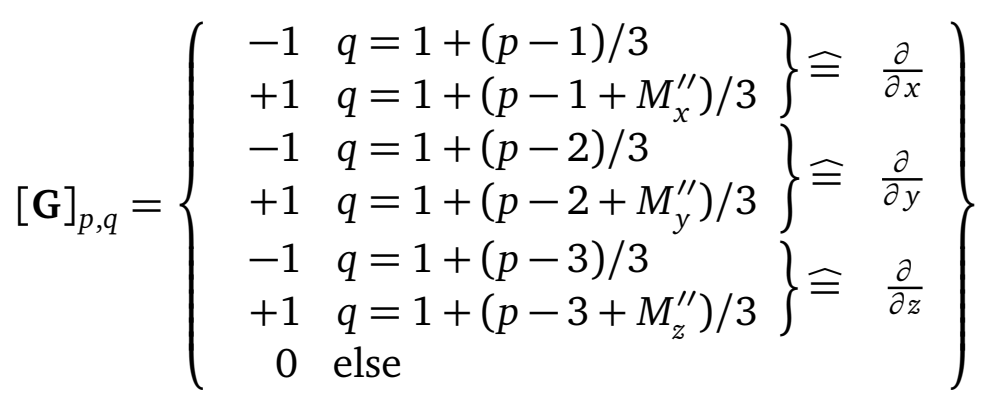

and $\left(1 \leq p \leq 3 N_{\text {cells }} ; 1 \leq q \leq N_{\text {cells }}\right)$

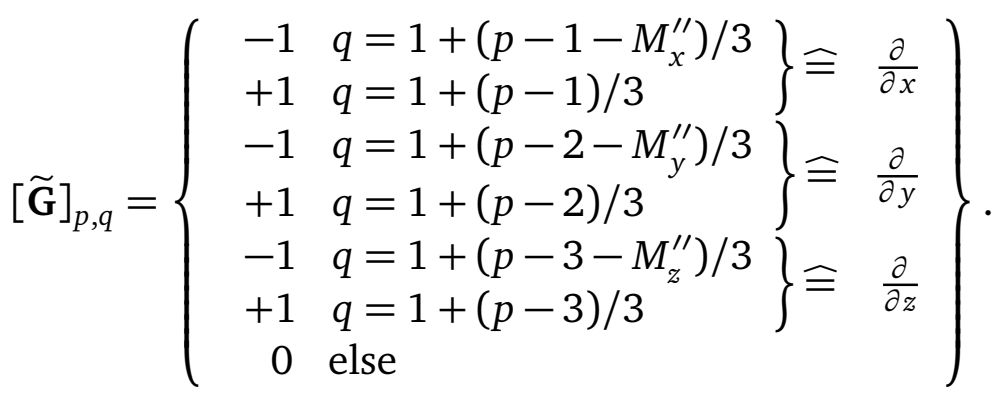


For further visualization, the structure of the matrices $\mathbf{C}$ and $\mathbf{S}$ is indicated for the example with $N_{x}=3, N_{y}=3$ and $N_{z}=4$ in the following:

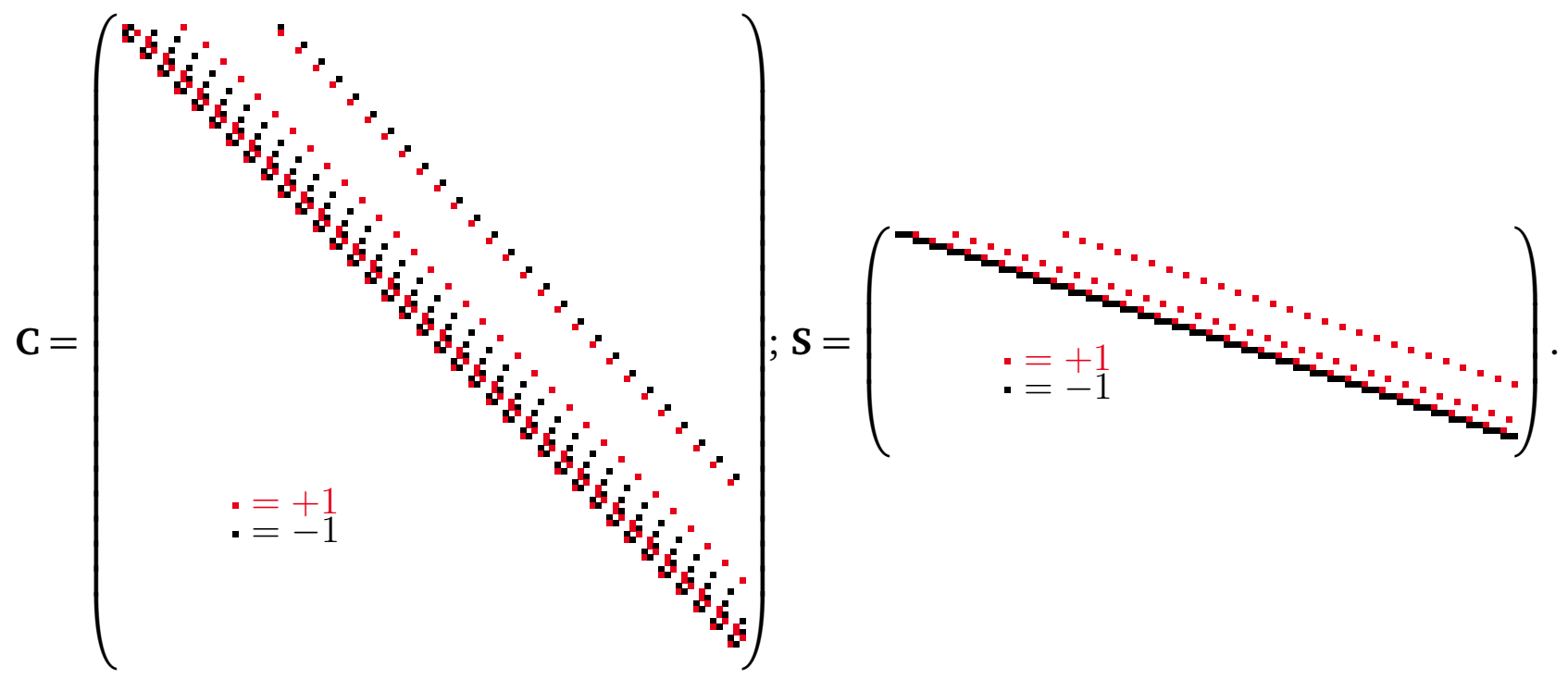

It shows that there are only a few nonzero entries located far away from the main diagonal for this ordering scheme. The same applies both for the corresponding matrices of the dual grid and the discretized matrices for the gradient operator. Note again that neither in the above definitions of the matrices nor in the visualization (3.57) matrix elements which are to be zeroed because they correspond to elements located outside the computation domain or due to boundary conditions are taken into account. By the way, since for an efficient parallel computation only the number of nonzeros in the off-diagonal blocks matters (cf. section 3.3), the actual ordering of the DOFs on each single node does not affect the overall computation performance (at least neglecting possible caching effects). Thus, one might want to combine different ordering schemes: For instance, scheme 2 on the large scale to partition the working domain onto different processes and scheme 1 locally for the ordering on each process.

For the sake of completeness, it should be mentioned that of course also the components of the material matrices and the matrices containing the lengths and the areas have to be adjusted according to the chosen ordering scheme. For diagonal matrices, neither advantages nor disadvantages arise due to the reordering. The situation changes, however, in case of non-diagonal material matrices such as for gyrotropic materials (cf. subsection 3.4.8). Here, a similar effect as for the topological matrices is achieved.

\subsubsection{General structure}

At first glance, the structure of the topological matrices of ordering 2 might seem to be very different from the clearly structured correspondents of ordering 1 . This is mainly due to the fact that for ordering scheme 2 it is not possible to express all topological matrices in terms of two classes of $\left(N_{\text {cells }} \times N_{\text {cells }}\right)$-submatrices. However, as will be shown in this paragraph, by introducing a more general set of matrices for the discretized differential operators, both ordering schemes can actually be expressed using the same notation.

Again, the general structure of the matrix $\mathbf{C}$ is considered at first. As was pointed out above (cf. Eq. (3.38)), each row of the matrix $\mathbf{C}$ gives the contour-integral on one facet, which can be 
decomposed into the sum of two differentials of the form $\pm v_{a}(n) \mp v_{a}\left(n+M_{b}\right),(a, b=x, y, z ; a \neq$ $b)$. For facets with orientation in $x$-direction, the discretized differential with respect to the to $y$-direction for the $z$-component (with positive sign) and the discretized differential with respect to the to $z$-direction for the $y$-component (with negative sign) are involved. Analogous relations are obtained for facets oriented in direction $y$ and $z$. This means that the complete matrix $\mathbf{C}$ can, in full generality, be decomposed into the sum of six discretized differential matrices ${ }^{\mathrm{C}} \mathbf{P}$ :

$$
\begin{aligned}
& \mathbf{C}={ }^{\mathrm{C}} \mathbf{P}_{y}^{(z)}-{ }^{\mathrm{C}} \mathbf{P}_{z}^{(y)} \quad\left(\widehat{\equiv} \text { contour around } A_{x}\right) \\
& +{ }^{\mathrm{C}} \mathbf{P}_{z}^{(x)}-{ }^{\mathrm{C}} \mathbf{P}_{x}^{(z)} \quad \text { ( } \text { contour around } A_{y} \text { ) } \\
& +{ }^{\mathrm{C}} \mathbf{P}_{x}^{(y)}-{ }^{\mathrm{C}} \mathbf{P}_{y}^{(x)} \quad\left(\widehat{\equiv} \text { contour around } A_{z}\right) \text {. }
\end{aligned}
$$

As before, the subscript refers to the direction with respect to which the differential is to be taken. The additional superscript in brackets gives the component of the subsequent vector on which the matrix $\mathbf{P}$ acts. For instance, ${ }^{C} \mathbf{P}_{x}^{(y)}$ denotes the discretized differential with respect to direction $x$ acting on all $y$-components of the subsequent vector. The matrices ${ }^{\mathrm{C}} \mathbf{P}_{b}^{(a)}$ must have the same dimension as the matrix $\mathbf{C}$, i.e. $\left(3 N_{\text {cells }} \times 3 N_{\text {cells }}\right)$. Yet, it is worth emphasizing that only one third of all rows of the matrices ${ }^{C} \mathbf{P}_{b}^{(a)}$ contains nonzero elements.

Looking at the structure of the matrix $\mathbf{S}$, equation (3.41) suggests that the integral associated with each row involves the sum of three differentials of the form $-v_{a}(n)+v_{a}\left(n+M_{a}\right),(a=$ $x, y, z)$. Hence, the most general form of the matrix $\mathbf{S}$ can be formulated as

$$
\mathbf{S}={ }^{\mathrm{S}} \mathbf{P}_{x}^{(x)}+{ }^{\mathrm{S}} \mathbf{P}_{y}^{(y)}+{ }^{\mathrm{S}} \mathbf{P}_{z}^{(z)}
$$

Keeping in mind that the dimension of the matrix $\mathbf{S}$ is $\left(N_{\text {cells }} \times 3 N_{\text {cells }}\right)$, for dimensional reasons alone it is clear that the matrix $\mathbf{S}$ cannot be composed of the same matrices $\mathbf{P}$ as the matrix C. In order not to be confused with the types of differential matrices, the matrices $\mathbf{P}$ carry an additional left superscript (C, S or G). Moreover, a third set of differential matrices ${ }^{\mathrm{G}} \mathbf{P}$ is required for the general representation of the matrix $\mathbf{G}$, the discretized analog of the gradient operator:

$$
\mathbf{G}={ }^{\mathrm{G}} \mathbf{P}_{x}+{ }^{\mathrm{G}} \mathbf{P}_{y}+{ }^{\mathrm{G}} \mathbf{P}_{z}
$$

For the dual grid the following definitions apply:

$$
\widetilde{\mathbf{C}}={ }^{\mathrm{C}} \widetilde{\mathbf{P}}_{y}^{(z)}-{ }^{\mathrm{C}} \widetilde{\mathbf{P}}_{z}^{(y)}+{ }^{\mathrm{C}} \widetilde{\mathbf{P}}_{z}^{(x)}-{ }^{\mathrm{C}} \widetilde{\mathbf{P}}_{x}^{(z)}+{ }^{\mathrm{C}} \widetilde{\mathbf{P}}_{x}^{(y)}-{ }^{\mathrm{C}} \widetilde{\mathbf{P}}_{y}^{(x)}
$$

for the curl operator,

$$
\widetilde{\mathbf{S}}={ }^{\mathrm{S}} \widetilde{\mathbf{P}}_{x}^{(x)}+{ }^{\mathrm{S}} \widetilde{\mathbf{P}}_{y}^{(y)}+{ }^{\mathrm{S}} \widetilde{\mathbf{P}}_{z}^{(z)}
$$

for the source operator and finally

$$
\widetilde{\mathbf{G}}={ }^{\mathrm{G}} \widetilde{\mathbf{P}}_{x}+{ }^{\mathrm{G}} \widetilde{\mathbf{P}}_{y}+{ }^{\mathrm{G}} \widetilde{\mathbf{P}}_{z}
$$

for the gradient operator. To sum up, all topological matrices can be expressed in terms of in total six different types of differential matrices. Yet, not all of the latter are independent of each other (cf. subsection 3.4.5.4). 
Table 3.1.: Parameters for ordering 1 and 2 for the representation of the topological matrices in terms of the general expressions.

\begin{tabular}{c|c|c|c|c|c|c}
\hline \hline & $M_{x}$ & $M_{y}$ & $M_{z}$ & $\Delta_{x}$ & $\Delta_{y}$ & $\Delta_{z}$ \\
\hline Ordering 1 & 1 & $N_{x}$ & $N_{x} N_{y}$ & 0 & $N_{\text {cells }}$ & $2 N_{\text {cells }}$ \\
Ordering 2 & 3 & $3 N_{x}$ & $3 N_{x} N_{y}$ & 0 & 1 & 2 \\
\hline \hline
\end{tabular}

\section{Explicit representation of the topological matrices in general form for ordering 1 and 2}

The expressions for the topological matrices given before hold true for any ordering of the FIT-DOFs. Yet, the structure of the involved differential matrices $\mathbf{P}$ and $\widetilde{\mathbf{P}}$ does depend on it. Explicit expressions for these matrices both for ordering 1 and 2 are presented in the following, starting with the ones for the primary grid.

The differential matrices for matrix $\mathbf{C}$ can be written as $(a, b, c=x, y$, or $z$ with $c \neq a ; c \neq b)$

$$
\left[{ }^{C} \mathbf{P}_{a}^{(b)}\right]_{p, q}=\left\{\begin{array}{rl}
-1 & q=p+\Delta_{b}-\Delta_{c} \\
+1 & q=p+\Delta_{b}-\Delta_{c}+M_{a} \\
0 & \text { else }
\end{array}\right\}
$$

where only rows with index $p=\Delta_{c}+1, \Delta_{c}+1+M_{x}, \Delta_{c}+1+2 M_{x}, \cdots, \Delta_{c}+1+\left(N_{\text {cells }}-1\right) M_{x}$ contain nonzero elements, whereas all remaining rows are identically zero. Consequently, only columns with index $q=\Delta_{b}+1, \Delta_{b}+1+M_{x}, \Delta_{b}+1+2 M_{x}, \cdots, \Delta_{b}+1+\left(N_{\text {cells }}-1\right) M_{x}$ contain nonzero elements. This definition holds both for ordering 1 and 2 . The only difference between these two are the values for the parameters $\Delta_{a}$ and $M_{a}$, which are given in table 3.1 and can be interpreted as follows. The quantities $M_{a}$ indicate the number by which the index increases if one proceeds one cell in direction $a$ and were already introduced separately as $M_{a}^{\prime}$ in subsection 3.4.5.1 in equation (3.37) for ordering 1 and as $M_{a}^{\prime \prime}$ in subsection 3.4.5.2 in equation (3.50) for ordering 2 . In contrast, the parameters $\Delta_{a}$ specify the change in the index when going from the $x$-component of one selected cell to the $y$-component $\left(\Delta_{y}\right)$ or $z$-component $\left(\Delta_{z}\right)$ of the same cell (i.e. $\Delta_{x}=0$ ). Note that $\Delta_{a}$ and $M_{a}$ are not independent of each other since the total of the index change starting from the $x$-component of the first cell (index 1) and ending at the z-component of the last cell (index $3 N_{\text {cells }}$ ) must always be $3 N_{\text {cells }}$. That is to say, the condition

$$
\begin{aligned}
& 1+\left(N_{x}-1\right) M_{x}+\left(N_{y}-1\right) M_{y}+\left(N_{z}-1\right) M_{z}+\Delta_{z}= \\
& 1+\left(N_{\text {cells }}-1\right) M_{x}+\Delta_{z} \stackrel{!}{=} 3 N_{\text {cells }}
\end{aligned}
$$

must be fulfilled. Moreover, since $2 \Delta_{y}=\Delta_{z}$ and $\Delta_{x}=0$ by definition, this condition fully determines the parameters $\Delta_{a}$.

Moreover, for the matrix $\mathbf{S}$ the differential matrices take the form $(a, b, c=x, y$, or $z)$

$$
\left[{ }^{\mathrm{S}} \mathbf{P}_{a}^{(a)}\right]_{p, q}=\left\{\begin{array}{rl}
-1 & q=1+M_{x}(p-1)+\Delta_{a} \\
+1 & q=1+M_{x}(p-1)+\Delta_{a}+M_{a} \\
0 & \text { else }
\end{array}\right\}
$$

where every row contains nonzero elements, that is to say, the row index $p$ is in the range from 1 to $N_{\text {cells. }}$. This implies that only columns with index $\Delta_{a}+1 \leq q \leq 1+M_{x}\left(N_{\text {cells }}-1\right)+\Delta_{a}$ contain 
nonzeros. Again, this expression is applicable to ordering 1 and 2 . The involved parameters are the same as introduced for the matrix $\mathbf{C}$ (cf. table 3.1). Finally, the differential matrices for matrix $\mathbf{G}$ are given by

$$
\left[{ }^{G} \mathbf{P}_{a}\right]_{p, q}=\left\{\begin{array}{rl}
-1 & q=1+\left(p-1-\Delta_{a}\right) / M_{x} \\
+1 & q=1+\left(p-1-\Delta_{a}+M_{a}\right) / M_{x} \\
0 & \text { else }
\end{array}\right\},
$$

with nonzero rows only in the range $\Delta_{a}+1 \leq q$. For the dual grid similar expressions are obtained. The differential matrices for matrix $\widetilde{\mathbf{C}} \operatorname{read}(a, b, c=x, y$, or $z$ with $c \neq a ; c \neq b)$

$$
\left[{ }^{\mathrm{C}} \widetilde{\mathbf{P}}_{a}^{(b)}\right]_{p, q}=\left\{\begin{array}{rl}
+1 & q=p+\Delta_{b}-\Delta_{c} \\
-1 & q=p+\Delta_{b}-\Delta_{c}-M_{a} \\
0 & \text { else }
\end{array}\right\}
$$

As for the matrix $\mathbf{C}$, only rows with index $p=\Delta_{c}+1, \Delta_{c}+1+M_{x}, \Delta_{c}+1+2 M_{x}, \ldots, \Delta_{c}+$ $1+\left(N_{\text {cells }}-1\right) M_{x}$ contain nonzero elements all remaining rows being identically zero. The corresponding expressions for $\widetilde{\mathbf{S}}$ take the form

$$
\left[{ }^{\mathrm{S}} \widetilde{\mathbf{P}}_{a}^{(a)}\right]_{p, q}=\left\{\begin{array}{rl}
+1 & q=1+M_{x}(p-1)+\Delta_{a} \\
-1 & q=1+M_{x}(p-1)+\Delta_{a}-M_{a} \\
0 & \text { else }
\end{array}\right\} .
$$

Finally, the differential matrices involved in the matrix $\widetilde{\mathbf{G}}$ are given by

$$
\left[{ }^{\mathrm{G}} \widetilde{\mathbf{P}}_{a}\right]_{p, q}=\left\{\begin{array}{rl}
+1 & q=1+\left(p-1-\Delta_{a}\right) / M_{x} \\
-1 & q=1+\left(p-1-\Delta_{a}-M_{a}\right) / M_{x} \\
0 & \text { else }
\end{array}\right\},
$$

where only non-vanishing rows are present in the range of row indices from $p=\Delta_{a}+1$ to $p=1+M_{x}\left(N_{\text {cells }}-1\right)+\Delta_{a}$. Of course, it is understood in all above definitions that the indices $p$ and $q$ are restricted to the dimension of the corresponding matrices. Again, note that additional zero rows or columns due to boundary conditions or elements allocated outside the computation domain have not been taken into account in the expressions mentioned before. These issues are addressed in subsection 3.4.7.1.

To sum up, in this section it has been shown that all the topological matrices can, in full generality, be decomposed into a sum of differential matrices, whose explicit form is determined by the ordering of the DOFs. Moreover, it is possible to find parameterized expressions for the involved differential matrices, which are identical both for ordering 1 (cf. subsection 3.4.5.1) and ordering 2 (cf. subsection 3.4.5.2). This shows manifestly that these two ordering schemes have indeed the same intrinsic characteristics. In particular, the clear structure of the topological matrices, one of the main benefits of ordering 1, is mainly preserved in ordering 2 though it may not be as obvious at first sight for the latter. In fact, regarding the implementation, the same structure of both ordering schemes even enables to construct the topological matrices for both orderings with the same code; for the selection of the wanted scheme it is sufficient to specify a few characteristic parameters (cf. table 3.1). Yet, individual, optimized codes might be preferred for the ease of implementation or for efficiency. Of course, as can easily be checked, applying the general expressions of subsection 3.4.5.3 results in exactly the same topological matrices as for the specific definitions of subsection 3.4.5.1 for ordering 1 or of subsection 3.4.5.2 for ordering 2 , respectively. 


\subsubsection{Properties of the topological matrices}

\section{Relations between topological matrices of the primary and dual grid}

As discussed in subsection 3.4.5.3, the topological matrices in the FIT can be written in terms of six different types of discretized differential operators. In this paragraph, it is shown that some of these are dependent on each other, which leads to some important relations between the topological matrices. These relations, which are presented in [52] for ordering 1, hold also for the expressions of the general ordering.

From the explicit form of the differential matrices for the matrices $\mathbf{C}$ (cf. Eq. (3.64)) and $\widetilde{\mathbf{C}}$ (cf. Eq. (3.68)) for ordering 1 and 2 in the general form, it follows directly

$$
{ }^{\mathrm{C}} \widetilde{\mathbf{P}}_{a}^{(b)}=-\left({ }^{\mathrm{C}} \mathbf{P}_{a}^{(c)}\right)^{T}
$$

Applying additionally the definitions (3.58) for the matrix $\mathbf{C}$ and (3.61) for the matrix $\widetilde{\mathbf{C}}$, one obtains the relation

$$
\widetilde{\mathbf{C}}=\mathbf{C}^{T} \text {. }
$$

Moreover, comparing the explicit form of the differential matrices for the matrices $\widetilde{\mathbf{S}}$ (cf. Eq. (3.69)) and G (cf. Eq. (3.67)) reveals

$$
{ }^{\mathrm{S}} \widetilde{\mathbf{P}}_{a}^{(a)}=-\left({ }^{\mathrm{G}} \mathbf{P}_{a}\right)^{T}
$$

and together with the definitions (3.62) for the matrix $\widetilde{\mathbf{S}}$ and (3.60) for the matrix $\mathbf{G}$

$$
\widetilde{\mathbf{S}}=-\mathbf{G}^{T}
$$

Analogously, the relations

$$
{ }^{\mathrm{S}} \mathbf{P}_{a}^{(a)}=-\left({ }^{\mathrm{G}} \widetilde{\mathbf{P}}_{a}\right)^{T}
$$

and consequently

$$
\mathbf{S}=-\widetilde{\mathbf{G}}^{T}
$$

are obtained.

The starting point for the derivation of the relations stated above were expressions applicable to ordering 1 and 2 without consideration of boundary conditions or elements outside the computation domain. However, all the results presented in this subsection still hold in full generality for any possible ordering scheme and also with additional zero rows and columns for the above reasons. The proof for the former can be found in appendix A.1, the latter is discussed in subsection 3.4.7.1. 


\section{Operator identities}

The following applies [52]:

$$
\begin{aligned}
& \mathbf{S C}=0, \\
& \widetilde{\mathbf{S C}}=0 .
\end{aligned}
$$

Equations (3.77) are the discretized analogs of the analytical operator identity $\operatorname{div} \operatorname{rot} \vec{V} \equiv 0$ on the primary and dual grid, respectively. For a proof based on local analysis of elementary cells, which is hence independent of the structure of the grid and of the ordering scheme, is referred to [52]. Also a second analytical operator identity rot grad $\phi \equiv 0$ is preserved in the FIT, which reads for the primary and dual grid

$$
\begin{aligned}
& \mathbf{C G}=0, \\
& \widetilde{\mathbf{C}} \widetilde{\mathbf{G}}=0,
\end{aligned}
$$

respectively. Equations (3.78) follow directly from the properties of the topological matrices together with the identities (3.77), since, e.g., for the primary, grid $\mathbf{C G}=-\widetilde{\mathbf{C}} \widetilde{\mathbf{S}}=-(\mathbf{S C})^{T}=0$ [52].

The fact that these analytical relations are still exactly fulfilled for the discretized field problem is of major importance regarding the robust solution of field problems based on the FIT.

\subsubsection{Boundary conditions}

The two most common types of boundary conditions are discussed in this subsection. These are the electric boundary conditions, which are characterized by vanishing tangential components of the electric field at the boundary, and magnetic boundary conditions, for which the tangential magnetic field components are absent. Whereas the former are essential, the latter are natural [52]. That is to say, magnetic boundary conditions are automatically satisfied without having to make particular adjustments. In contrast, electric boundary conditions have to be imposed explicitly on the solution. This is done by explicitly zeroing those rows of the system matrix that are associated with tangential electric field components on the boundary. More details, particularly regarding symmetry considerations and the practical implementation, are discussed in the following subsection in a more general context.

\subsubsection{Reduced grid}

As mentioned before, all the vectors in the standard FIT have components which are associated with elements where the field solution must a priori be zero due to three possible reasons: Firstly, the elements are allocated outside the computation domain (cf. subsection 3.4.5.1, particularly Fig. 3.4); secondly, due to the imposed boundary conditions (cf. subsection 3.4.6); or thirdly, the elements are allocated in perfect electric conductor (PEC) cells or wires. As a short hand, the term pseudo-degree of freedom (pseudo-DOF) will be used for such vanishing components in the following. The resulting zero structure is categorized for the different vectors and topological matrices in subsection 3.4.7.1. Basically, there are two ways how to address the issue regarding the pseudo-DOFs: The common standard is to just set those particular elements 
Table 3.2.: Classification of vectors regarding the zero structure in the FIT.

\begin{tabular}{r|l}
\hline \hline type & vectors \\
\hline$E$-field-like & $\widehat{\mathbf{e}}, \widehat{\mathbf{d}}, \mathbf{D}_{\varepsilon}, \mathbf{D}_{\kappa}, \widehat{\mathbf{j}}, \mathbf{D}_{L}, \widetilde{\mathbf{D}}_{A}$ \\
$H$-field-like & $\widehat{\mathbf{h}}, \widehat{\mathbf{b}}, \mathbf{D}_{\mu}, \mathbf{D}_{v}, \widetilde{\mathbf{D}}_{L}, \mathbf{D}_{A}$ \\
$\begin{array}{r}\text { primary node-like } \\
\text { dual node-like }\end{array}$ & $\mathbf{q}$ \\
$\mathbf{q}_{\mathrm{m}}$ \\
\hline \hline
\end{tabular}

explicitly to zero but keep them in the data structure. Another approach is to remove them completely, which will be referred to as "reduced grid" and which is pursued in this work. The advantages and disadvantages of the two alternatives are weighed up in subsection 3.4.7.2. Its implementation details are discussed in subsection 3.4.7.3 to close this section.

\subsubsection{Zero structure of vectors and topological matrices}

All the expressions for the vectors and matrices obtained so far are, strictly speaking, only valid in the bulk of the computation domain. As pointed out above, at its boundary or due to PEC-cells or -wires, however, some special treatment is required. Of course, this issue arises regardless of the used ordering scheme. Before coming to the actual discussion of the treatment of these zero values in the topological matrices, it is worth to have a look at the zero structure of the vectors in the FIT.

\section{Classification of vectors}

Regarding the zero pattern, the vectors in the FIT can be classified into four different types as listed in table 3.2. According to this, vectors are referred to as $E$-field-like, $H$-field-like, primary node-like and dual node-like throughout this work. Note that, though the DOFs are allocated on the primary or dual grid in six different ways (cf. subsection 3.4.2), there are only four distinct vector types regarding the zero pattern. This is by virtue of the constitutive relations (cf. section 3.4.4), which connect DOFs from the primary grid to those of the dual grid.

Clearly, in case that a material matrix exhibits non-diagonal elements, as for, e.g., gyrotropic materials (cf. subsection 3.4.8), the zero pattern of both its rows and columns must be the same as for the purely diagonal correspondent.

\section{Zero structure of topological matrices}

As is pointed out above, all the vectors in the FIT must have the correct zero pattern. When solving field problems, this requirement can be met by simply zeroing rows of the topological matrices associated with one of the vector components to be zeroed. Note, however, that by doing so the symmetric structure of the topological matrices is spoiled, which is a crucial requirement for their properties discussed in subsection 3.4.5.4. Yet, the symmetry can be restored by additionally setting those columns to zero that are associated with pseudo-DOFs of the vector to be multiplied with. Hence, the rows and columns of all matrices in the FIT should exhibit the zero pattern of one of the four types as for the vectors, too. Details for all topological matrices are given table 3.3.

To formulate the above said in a mathematically more stringent way, the diagonal matrices $\mathbf{D}_{0}^{A}$ for $A$-like vectors are introduced, where $A$ denotes either $E$-field, $H$-field, primary node or 
Table 3.3.: Zero pattern of the rows and columns of topological matrices in the FIT.

\begin{tabular}{c|c|c}
\hline \hline & rows & columns \\
\hline $\mathbf{C}$ & $H$-field-like & $E$-field-like \\
$\widetilde{\mathbf{C}}$ & $E$-field-like & $H$-field-like \\
$\mathbf{S}$ & dual node-like & $H$-field-like \\
$\widetilde{\mathbf{S}}$ & primary node-like & $E$-field-like \\
$\mathbf{G}$ & $E$-field-like & primary node-like \\
$\widetilde{\mathbf{G}}$ & $H$-field-like & dual node-like \\
\hline \hline
\end{tabular}

dual node. Their diagonal entries $\left[\mathbf{D}_{0}^{A}\right]_{p, p}$ are either 1 (for ordinary elements) or 0 if this entry corresponds to a pseudo-DOF. For the rest of this paragraph topological matrices without consideration of these additional zero values are denoted by the subscript $\overline{0}$; the ones including the additional zero elements miss this subscript. That is to say, employing the notation introduced before, the following applies:

$$
\begin{aligned}
\mathbf{C} & =\mathbf{D}_{0}^{\mathrm{H}} \mathbf{C}_{\overline{0}} \mathbf{D}_{0}^{\mathrm{E}}, \\
\widetilde{\mathbf{C}} & =\mathbf{D}_{0}^{\mathrm{E}} \widetilde{\mathbf{C}}_{\overline{0}} \mathbf{D}_{0}^{\mathrm{H}}, \\
\mathbf{S} & =\mathbf{D}_{0}^{\text {dual }} \mathbf{S}_{\overline{0}} \mathbf{D}_{0}^{\mathrm{H}}, \\
\widetilde{\mathbf{S}} & =\mathbf{D}_{0}^{\text {primary }} \widetilde{\mathbf{S}}_{\overline{0}} \mathbf{D}_{0}^{\mathrm{E}}, \\
\mathbf{G} & =\mathbf{D}_{0}^{\mathrm{E}} \mathbf{G}_{\overline{0}} \mathbf{D}_{0}^{\text {primary }}, \\
\widetilde{\mathbf{G}} & =\mathbf{D}_{0}^{\mathrm{H}} \widetilde{\mathbf{G}}_{\overline{0}} \mathbf{D}_{0}^{\text {dual }} .
\end{aligned}
$$

The matrices multiplied from the left effectively zero appropriate rows whereas the ones multiplied from the right remove nonzero entries column-wise. Note again that the matrices multiplied from the left are necessary to ensure the right zero pattern of the resulting vector. The ones multiplied from the right are, however, not strictly required at this point. The reason for this is that, given a vector to be multiplied with in the correct zero pattern, they remove only these elements of the topological matrices which would access vector components which are zero anyway. Yet, the matrices multiplied from the right are important to restore the structure of the topological matrices so that their relations presented in subsection 3.4.5.4 are fulfilled manifestly.

Equations (3.79) should be understood rather as a helpful notation for theoretical considerations than as an implementation instruction. For efficiency the zero entries should not be assembled or even, as for the reduced grid, removed completely from the beginning.

In common field problems correct field solutions are still obtained with the FIT even if the additional zero values are not taken into account in the topological matrices explicitly. This is due to the fact that the topological matrices are usually accompanied by material matrices, see, e.g., the curl-curl equation (3.5). If it is ensured that the material matrices already exhibit the correct zero pattern, then they take on the role of the diagonal matrices $\mathbf{D}_{0}^{A}$ and hence also ensure the desired structure of the result vectors. Nevertheless, throughout this work all topological matrices are constructed with the full zero pattern or alternatively as reduced matrices where all rows and columns with only vanishing elements are removed completely as discussed in the remainder of this section. 


\subsubsection{Advantages and disadvantages of the reduced grid}

For the decision making whether or not to remove the pseudo-DOFs completely, the following aspects should be considered.

\section{Disadvantages of the reduced grid}

- Ease of implementation: Among the main advantages of keeping the pseudo-DOFs is clearly the ease of implementation since the band structure of the matrices is not spoiled. In contrast, in the reduced grid this simple band structure is not retained. This means, for instance, that it is more involved to tell which columns contain nonzero elements for the construction of the topological matrices. What is more, debugging the code is easier for the non-reduced grid. This should not be underestimated, particularly, for the parallel implementation.

- Computational costs: For the same, above-mentioned reason, some computational overhead for the calculation of the nonzero indices is introduced. Note, however, that this only matters for the construction of matrices (such as the topological matrices or the permeability tensor), not for the usage of already assembled matrices. As the time spent for the construction of matrices is usually very small compared with the solution of the actual field problem, this is not a severe issue. However, if shell matrices without explicit storage of the matrices are used, the computational overhead may become significant since the indices of the nonzero elements have to be re-computed at every matrix-vector multiplication.

- Memory requirements: Furthermore, since for the construction of the matrices the information is required how many indices have been removed before a specific index, some additional information about original indices of the pseudo-DOFs is stored.

The actual additional computational costs as well as memory requirements depend strongly on the chosen implementation, which is discussed in detail in subsection 3.4.7.3.

\section{Advantages of the reduced grid}

- Computational costs: As mentioned above, the reduced grid introduces some computational overhead for the construction of matrices on the one hand. On the other hand, a smaller number of matrix elements has to be assembled in the end. As the actual assembly is the most expensive part for the construction of sparse matrices, this usually overcompensates the negative contribution. Additionally, what is more important, also the performance for actual computations involving the already assembled matrices is enhanced. Particularly for the solution of systems of equations with direct solvers the computational cost is reduced since some equations are removed. For iterative solvers, the situation is different. Here, only a very low computational effort would be required to solve the removed lines as they are just trivial equations. This means that for the usage of iterative solvers the benefit is usually only thanks to the slightly enhanced performance of the matrix-vector product since one does not have to loop over rows of pseudo-DOFs. Notwithstanding, the positive impact on the performance might be significant in case that the entries associated with PEC-cells are not explicitly zeroed (and hence are assembled) in the topological matrices as in the standard FIT (see also next item). 
- Memory requirements: The required memory for the storage of matrices and vectors is reduced, which scales with the number of pseudo-DOFs. An estimate how many pseudoDOFs actually exist is given in subsection 3.4.7.3. In more detail, regarding the vectors the saving on memory is directly proportional to the number of removed elements as the vector data is stored in dense arrays. That is, the required memory reduces by 8 bytes (16 bytes) for double precision real (complex) numbers for each pseudo-DOF and vector. With regard to matrices, the actual saving on memory depends on various factors. Assuming a CSR matrix format as introduced in section 3.3, the memory requirements shrink by at least 4 bytes for a sequential matrix format or 8 bytes for a parallel format for each removed row, even if no entry is allocated in that row at all. Moreover, if the usage of a direct solver is desired for a system matrix, which still includes the pseudo-DOFs, one additional nonzero entry has to be set at all main diagonal elements associated with the pseudo-DOFs to ensure an explicitly non-singular system. Consequently, in the reduced grid additional $4+8$ bytes $(4+16$ bytes) are saved per pseudo-DOF for double precision real (complex) numbers. Furthermore, the elements associated with PEC-cells are usually not set zero in the topological matrices of standard FIT. It is only when multiplied with the material matrices that the correct zero pattern of the field vectors is ensured (cf. last paragraph in subsection 3.4.7.1). As a consequence, the explicitly constructed system matrix contains many allocated entries which are actually identically zero. Yet, even more importantly, its factorizing, e.g., for preconditioning, is not only computationally more expensive but also leads to much more fill-in and hence a significantly increased memory requirement.

- Load balancing: Once the reduced matrices are constructed, the reduced grid results in algorithms with enhanced load balancing. This is due to the fact that the pseudoDOFs, especially those due to elements outside the computation domain, are not evenly distributed across all processes for the non-reduced grid. Hence, the last process usually owns significantly less nonzero entries than, e.g., the first one. Given that the number of floating point operations one processing unit has to perform is more or less proportional to the number of nonzeros of the system matrix owned by this process, the workload tends to be poorly balanced. Removing the pseudo-DOFs, however, enables to provide an almost ideal load balancing (cf. Fig. 3.6). For completeness, it is noted that a similar effect might be achieved by employing PETSc's built-in option PCREDISTRIBUTE [47], which redistributes already assembled matrices evenly on different processes. This was, however, not tested in this thesis.

- Memory allocation: The reduced grid provides a noticeable speed-up for the memory allocation at the time the preconditioner for the solution of the Jacobi-Davidson correction equation (cf. Eq. (3.158)) is constructed. The reason for this is that the preconditioner is computed for the system matrix which is shifted by a scaled unit matrix. Since it is not possible to explicitly preallocate memory for vanishing diagonal elements when using PETSc-routines for sparse matrix-matrix multiplication, the consequence is that additional memory has to be allocated multiple times later on. This is, however, known to be an expensive operation [47]. The complete removal of the pseudo-DOFs is one effective way of solving this issue, which eventually results in a tremendous performance boost.

Taking all these points into consideration one comes to the conclusion that the usage of the reduced grid on the one hand complicates the process of constructing the matrices used in the 

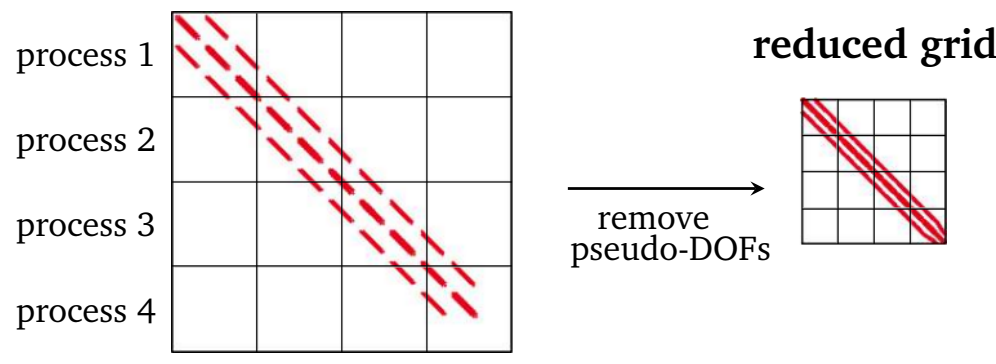

Figure 3.6.: Typical structure of a system matrix without removal of the pseudo-DOFs (left) and for the reduced grid (right) for ordering scheme 2. In the illustrated example a parallel computation with four processes is assumed. The nonzero DOFs are much more evenly distributed for the reduced grid, which leads to an improved load balancing.

FIT. On the other hand, it results in a significantly enhanced performance and reduced memory requirements for the solution of realistic field problems, which is even more evident when the discretized model contains a large number of PEC-cells (cf. also subsection 3.4.7.3). That is, if one is concerned about high-performance computations, it is recommended to remove all pseudo-DOFs explicitly.

In this work, the implementation is optimized for the efficient usage of the reduced grid. Yet, additionally a fully functional version without the removal of the pseudo-DOFs is available, which can be activated with a compiler flag for debugging purposes. Thus, one can benefit from the before-mentioned advantages of the reduced grid without loosing the advantage of efficient debugging. 


\subsubsection{Implementation details for the reduced grid}

\section{Number of pseudo-DOFs}

As discussed in detail in the previous subsection, the performance enhancement directly depends on the number of pseudo-DOFs to be removed in the reduced grid. For its determination a case distinction for the four different vector types as introduced in subsection 3.4.7.1 is necessary. A grid with dimension $N_{x} \times N_{y} \times N_{z}$ is assumed. In general, the total number of pseudo-DOFs $N_{\text {pseudo-DOFs }}^{A}$ for $A$-like vectors is the sum of the contribution of the elements outside the computation domain $N_{\text {outside }}^{A}$, the one due to boundary conditions $N_{\mathrm{BC}}^{A}$ and the one due to elements inside PEC-cells (or -wires) $N_{\mathrm{PEC}}^{A}$, i.e.

$$
N_{\text {pseudo-DOFs }}^{A}=N_{\text {outside }}^{A}+N_{\mathrm{BC}}^{A}+N_{\mathrm{PEC}}^{A} \cdot
$$

For $E$-field-like vectors one finds

$$
N_{\text {outside }}^{\mathrm{E}}=N_{y} N_{z}+N_{x} N_{z}+N_{x} N_{y}
$$

and

$$
\begin{aligned}
& N_{\mathrm{BC}}^{\mathrm{E}}=\left(N_{y}-1\right) N_{z}+\left(N_{z}-1\right) N_{y} \quad\left(\text { if } E_{t}=0 \text { on } x_{\min }\right) \\
& \left.+\left(N_{y}-1\right) N_{z}+\left(N_{z}-1\right) N_{y} \quad \text { (if } E_{t}=0 \text { on } x_{\max }\right) \\
& \left.+\left(N_{x}-1\right) N_{z}+\left(N_{z}-1\right) N_{x} \quad \text { (if } E_{t}=0 \text { on } y_{\min }\right) \\
& \left.+\left(N_{x}-1\right) N_{z}+\left(N_{z}-1\right) N_{x} \quad \text { (if } E_{t}=0 \text { on } y_{\max }\right) \\
& +\left(N_{x}-1\right) N_{y}+\left(N_{y}-1\right) N_{x} \quad\left(\text { if } E_{t}=0 \text { on } z_{\text {min }}\right) \\
& \left.+\left(N_{x}-1\right) N_{y}+\left(N_{y}-1\right) N_{x} \quad \text { (if } E_{t}=0 \text { on } z_{\max }\right) \\
& -\left(N_{z}-1\right) \quad\left(\text { if } E_{t}=0 \text { on } x_{\min } \text { and on } y_{\min }\right. \text { ) } \\
& -\left(N_{z}-1\right) \quad\left(\text { if } E_{t}=0 \text { on } x_{\min } \text { and on } y_{\max }\right) \\
& -\left(N_{y}-1\right) \quad\left(\text { if } E_{t}=0 \text { on } x_{\min } \text { and on } z_{\min }\right. \text { ) } \\
& -\left(N_{y}-1\right) \quad\left(\text { if } E_{t}=0 \text { on } x_{\min } \text { and on } z_{\max }\right. \text { ) } \\
& -\left(N_{z}-1\right) \quad \text { (if } E_{t}=0 \text { on } x_{\max } \text { and on } y_{\min } \text { ) } \\
& -\left(N_{z}-1\right) \quad\left(\text { if } E_{t}=0 \text { on } x_{\max } \text { and on } y_{\max }\right. \text { ) } \\
& -\left(N_{y}-1\right) \quad\left(\text { if } E_{t}=0 \text { on } x_{\max } \text { and on } z_{\min }\right. \text { ) } \\
& -\left(N_{y}-1\right) \quad\left(\text { if } E_{t}=0 \text { on } x_{\max } \text { and on } z_{\max }\right. \text { ) } \\
& -\left(N_{x}-1\right) \quad\left(\text { if } E_{t}=0 \text { on } y_{\min } \text { and on } z_{\min }\right) \\
& -\left(N_{x}-1\right) \quad\left(\text { if } E_{t}=0 \text { on } y_{\min } \text { and on } z_{\max }\right) \\
& -\left(N_{x}-1\right) \quad\left(\text { if } E_{t}=0 \text { on } y_{\max } \text { and on } z_{\min }\right) \\
& -\left(N_{x}-1\right) \quad\left(\text { if } E_{t}=0 \text { on } y_{\max } \text { and on } z_{\max }\right. \text { ) }
\end{aligned}
$$

However, the number $N_{\text {PEC }}^{\mathrm{E}}$ cannot be estimated exactly a priori because it depends not only on the pure number of PEC-cells $N_{\mathrm{PEC}}$ and PEC-wires but also on their actual position. For instance, each isolated PEC-cell in the middle of the computation domain would result in 12 additional pseudo-DOFs whereas two neighboring PEC-cells would not add 24 but only 20 pseudo-DOFs 
since 4 edges are part of both cells. Similarly, also the imposed boundary conditions modify the resulting number of additional pseudo-DOFs of PEC-cells located at the boundary of the computation domain. In order to find the exact number $N_{\mathrm{PEC}}^{\mathrm{E}}$ (and eventually the total $N_{\text {pseudo-DOFs }}^{\mathrm{E}}$ ) in practice, the employed algorithm counts the number "experimentally" in a preprocessing step, in which it checks for each element whether or not it is a pseudo-DOF. Nevertheless, for the following considerations it is sufficient to look at its asymptotic behavior in the limit of a large number of mesh cells, which is described by

$$
N_{\mathrm{PEC}}^{\mathrm{E}} \simeq 3 N_{\mathrm{PEC}}
$$

Analogously, for $H$-field-like vectors it is

$$
\begin{aligned}
N_{\text {outside }}^{\mathrm{H}}=2\left(\left(N_{x}-1\right) \times\left(N_{y}-1\right)+\left(N_{x}-1\right) \times\left(N_{z}-1\right)+\left(N_{y}-1\right)\right. & \left.\times\left(N_{z}-1\right)\right)+ \\
& +3\left(N_{x}+N_{y}+N_{z}-2\right)
\end{aligned}
$$

and

$$
\begin{aligned}
& N_{\mathrm{BC}}^{\mathrm{H}}=\left(N_{y}-1\right) \times\left(N_{z}-1\right) \quad\left(\text { if } E_{t}=0 \text { on } x_{\min }\right) \\
& +\left(N_{y}-1\right) \times\left(N_{z}-1\right) \quad\left(\text { if } E_{t}=0 \text { on } x_{\max }\right) \\
& +\left(N_{x}-1\right) \times\left(N_{z}-1\right) \quad\left(\text { if } E_{t}=0 \text { on } y_{\min }\right) \\
& +\left(N_{x}-1\right) \times\left(N_{z}-1\right) \quad\left(\text { if } E_{t}=0 \text { on } y_{\max }\right)^{\circ} \\
& +\left(N_{x}-1\right) \times\left(N_{y}-1\right) \quad\left(\text { if } E_{t}=0 \text { on } z_{\min }\right) \\
& +\left(N_{x}-1\right) \times\left(N_{y}-1\right) \quad\left(\text { if } E_{t}=0 \text { on } z_{\max }\right)
\end{aligned}
$$

For the same reasons as for E-field-like vectors, the contribution due to PEC-cells can only be estimated asymptotically for a fine mesh as

$$
N_{\mathrm{PEC}}^{\mathrm{H}} \simeq 3 N_{\mathrm{PEC}} \cdot
$$

Regarding dual nodes there is no contribution due to boundary conditions. Thus, the number of pseudo-DOFs is just the sum of the number of cells outside the computation domain, which is given by

$$
\begin{array}{r}
N_{\text {outside }}^{\text {dual }}=\left(N_{x}-1\right) \times\left(N_{y}-1\right)+\left(N_{x}-1\right) \times\left(N_{z}-1\right)+\left(N_{y}-1\right) \times( \\
\left(N_{z}-1\right)+ \\
+ \\
+N_{x}+N_{y}+N_{z}-2
\end{array}
$$


and the number of PEC-cells, that is, $N_{\mathrm{PEC}}^{\text {dual }}=N_{\mathrm{PEC}}$. In contrast, there are no primary nodes outside the computation domain, i.e. $N_{\text {outside }}^{\text {primary }}=0$, but the imposed boundary conditions lead to

$$
\begin{aligned}
& N_{\mathrm{BC}}^{\text {primary }}=N_{y} \times N_{z}+N_{z} \times N_{y} \quad\left(\text { if } E_{t}=0 \text { on } x_{\min }\right) \\
& +N_{y} \times N_{z}+N_{z} \times N_{y} \quad \text { (if } E_{t}=0 \text { on } x_{\max } \text { ) } \\
& +N_{x} \times N_{z}+N_{z} \times N_{x} \quad \text { (if } E_{t}=0 \text { on } y_{\text {min }} \text { ) } \\
& +N_{x} \times N_{z}+N_{z} \times N_{x} \quad \text { (if } E_{t}=0 \text { on } y_{\text {max }} \text { ) } \\
& +N_{x} \times N_{y}+N_{y} \times N_{x} \quad\left(\text { if } E_{t}=0 \text { on } z_{\text {min }}\right. \text { ) } \\
& +N_{x} \times N_{y}+N_{y} \times N_{x} \quad \text { (if } E_{t}=0 \text { on } z_{\text {max }} \text { ) } \\
& -N_{z} \quad \text { (if } E_{t}=0 \text { on } x_{\min } \text { and on } y_{\min } \text { ) } \\
& -N_{z} \quad \text { (if } E_{t}=0 \text { on } x_{\min } \text { and on } y_{\text {max }} \text { ) } \\
& -N_{y} \quad\left(\text { if } E_{t}=0 \text { on } x_{\min } \text { and on } z_{\min }\right) \\
& -N_{y} \quad\left(\text { if } E_{t}=0 \text { on } x_{\text {min }} \text { and on } z_{\text {max }}\right. \text { ) } \\
& -N_{z} \quad \text { (if } E_{t}=0 \text { on } x_{\max } \text { and on } y_{\min } \text { ) } \\
& -N_{z} \quad \text { (if } E_{t}=0 \text { on } x_{\text {max }} \text { and on } y_{\text {max }} \text { ) } \\
& -N_{y} \quad \text { (if } E_{t}=0 \text { on } x_{\max } \text { and on } z_{\min } \text { ) } \\
& -N_{y} \quad \text { (if } E_{t}=0 \text { on } x_{\max } \text { and on } z_{\max } \text { ) } \\
& -N_{x} \quad\left(\text { if } E_{t}=0 \text { on } y_{\min } \text { and on } z_{\min }\right. \text { ) } \\
& -N_{x} \quad \text { (if } E_{t}=0 \text { on } y_{\min } \text { and on } z_{\max } \text { ) } \\
& -N_{x} \quad \text { (if } E_{t}=0 \text { on } y_{\max } \text { and on } z_{\min } \text { ) } \\
& -N_{x} \quad \text { (if } E_{t}=0 \text { on } y_{\max } \text { and on } z_{\max } \text { ) }
\end{aligned}
$$

pseudo-DOFs. Again, the number of pseudo-DOFs due to PEC-cells and -wires has to be determined in a preprocessing step. One finds the asymptotic relation

$$
N_{\text {PEC }}^{\text {primary }} \simeq N_{\text {PEC }} \text {. }
$$

Taking into account that the total number of all DOFs is $N_{\mathrm{DOFs}}^{\mathrm{E}}=N_{\mathrm{DOFs}}^{\mathrm{H}}=3 N_{\mathrm{DOFs}}^{\text {dual }}=3 N_{\mathrm{DOFs}}^{\text {primary }}=$ $3 N_{x} N_{y} N_{z}$, the ratio of pseudo-DOFs due to elements outside the computation domain and due to boundary conditions to the total number of DOFs scales asymptotically as

$$
\frac{N_{\text {outside }}^{A}+N_{\mathrm{BC}}^{A}}{N_{\text {DOFs }}^{A}} \simeq \frac{1}{N},
$$

where $N$ is the minimum of $N_{x}, N_{y}$ and $N_{z}$. Hence, for a fine grid with $N \gg 1$ the contribution of these two components becomes negligibly small. In contrast, as the number of PEC-cells also scales as $N_{x} N_{y} N_{z}$ for a fixed geometry, i.e. $N_{\mathrm{PEC}}^{A} \simeq N_{x} N_{y} N_{z}$, one obtains

$$
\frac{N_{\text {PEC }}^{A}}{N_{\text {DOFs }}^{A}} \backsim \text { constant. }
$$

That is to say, the relative contribution of the pseudo-DOFs due to PEC-cells asymptotically stays the same. Consequently, as already stated in the previous subsection, the benefits of the reduced grid are more obvious if the model to be discretized exhibits large amounts of PEC material. This is, for instance, the case for the simulation of the GSI SIS 18 cavity (cf. section 4.3). 


\section{Different approaches for the determination of indices associated with the reduced grid}

In practice it is often required to determine the index of a particular element in the reduced grid given an index of the non-reduced grid. This is, e.g., the case when the reduced matrices are constructed. To this end, the number of pseudo-DOFs that have already been removed before this element, $N_{\text {removed }}$, has to be known. Three different approaches, which all serve this purpose but behave differently with respect to performance and requirements on memory, are considered:

1. Storage of one flag ( 1 bit), which is 1 if the index is removed in the reduced grid (i.e. is a pseudo-DOF) and is 0 otherwise, for each non-reduced index as a bit-array. To determine $N_{\text {removed }}$, all bits to the right of this bit have to be counted, which takes linear time $\left(\mathscr{O}\left(N_{\text {DOFs }}^{A}\right)\right)$. Memory requirement: $N_{\text {DOFs }}^{A} / 8$ byte

2. Storage of all non-reduced indices that are removed in the reduced grid as an integer array. To determine the number $N_{\text {removed }}$ of removed elements before a particular element with non-reduced index $p$, a binary search algorithm is used to find the index $i$ of the (sorted) integer-array $a$ such that $a[i]<p \leq a[i+1]$, where $a[i]$ denotes the $i$-th element of $a$; $N_{\text {removed }}$ then equals the found index $i$. Note that a binary search locates one element in logarithmic time, i.e. it has an average case performance of $\mathscr{O}\left(\log \left(N_{\text {pseudo-DOFs }}^{A}\right)\right)$ [54]. Memory requirement: $4 N_{\text {pseudo-DOFs }}^{A}$ byte

3. Explicit storage of $N_{\text {removed }}$ for each non-reduced index as an integer-array. Hence, it takes only a constant, very short time to get $N_{\text {removed }}$. Memory requirement: $4 N_{\text {DOFs }}^{A}$ byte

Comparing these three approaches, it shows that the first one has the lowest memory requirement up to a moderate number of mesh cells. Yet, number two requires fewest memory for a large number of cells (with roughly $\gtrsim 10^{6}$, the exact number particularly depends on the number of PEC-cells). Approach three on the one hand always exhibits by far the highest requirement on memory, on the other hand it is the fastest one for a random access of indices. Still with respect to runtime, approach one is found to be several orders of magnitude slower than number two for a large number of DOFs and a random access of the indices. Besides of that, indices have frequently to be accessed not in random but in ascending order. In this case, one can easily take advantage of it with the effect that it takes only a constant, very short time to access the subsequent index for any of the three approaches. Taking all this into consideration, approach two seems to be the best compromise between performance and memory requirements. Consequentially, it is used throughout this work.

Though the obtained system matrices for the reduced grid enhance the performance of parallelized codes (cf. subsection 3.4.7.2), the task of determining the associated index in the reduced grid itself does not scale well. The main issue here is related to the fact that a process owns all columns (within the range of owned rows) of a matrix. This means that every process has to be able to determine a reduced index for any given global index, also including indices of elements, whose associated rows are not owned by this process. Yet, due to the relations of the matrices of the primary and dual grid (cf. subsection 3.4.5.4), it is sufficient to handle this only for $E$ - and $H$-field-like vectors since the gradient matrices, which involve primary nodeor dual node-like columns, can be obtained by taking the transpose of the source matrices (cf. subsection 3.4.5.4). Thus, in the implementation of this work every process stores all indices of pseudo-DOFs for $E$ - and $H$-field-like vectors. Since the memory overhead is small relative to the total used memory, this is accepted in view of the logarithmic runtime of approach two 


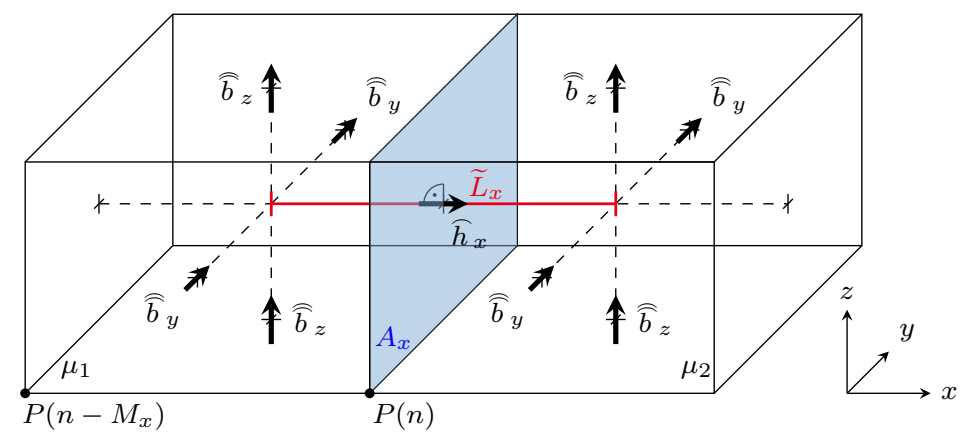

Figure 3.7.: Intersection of the dual edge $\widetilde{L}_{x}$ with the primary facet $A_{x}$. For the construction of the inverse permeability matrix, averaging of the inverse permeability along the dual edge is performed. [59]

and since taking into account that storing only a portion of the indices and communicating the missing indices would be much slower. In contrast, for primary node- and dual node-like vectors only indices of pseudo-DOFs associated with this process are stored. Moreover, if memory was the limiting factor of the used code on a particular machine, the additionally allocated memory for the construction of reduced matrices could be freed before starting the actual solver and afterwards be re-allocated for post-processing.

\subsubsection{Modeling of gyrotropic materials in frequency domain}

The above described standard FIT is restricted to diagonal anisotropic materials. Yet, in order to fully take into account the tensor characteristics of gyrotropic materials, an extension is required. For the time domain, this was already thoroughly treated in [55-57], whereas the problem of the simulation of gyrotropic substances in frequency domain was picked up in [58]. The modeling of gyrotropic materials in frequency domain including the derivation of explicit expressions for the material matrices is discussed in this subsection starting with gyromagnetic materials followed by gyroelectric ones. The main parts of this subsection are published in [59].

\subsubsection{Modeling of gyromagnetic materials}

In order to derive the form of the inverse permeability tensor for the FIT, we start from the constitutive relation between the magnetic induction $\vec{B}$ and the magnetic field $\vec{H}$ for gyromagnetic materials in the form (3.1) with its equivalence in FIT-notation

$$
\widehat{\mathbf{h}}=\mathbf{M} \stackrel{\widehat{v}}{\widehat{\mathbf{b}}}
$$

The task is then to find an explicit expression for the non-diagonal inverse permeability tensor $\mathbf{M}_{\stackrel{v}{*}}$. To this end, the magnetic voltage $\widehat{h}_{x}(n)$ along the dual edge $\widetilde{L}_{x}(n)$ is considered (cf. 
Fig. 3.7). The primary cells given by the nodes $P\left(n-M_{x}\right)^{2}$ and $P(n)$ are assumed to contain material whose magnetic properties can be described by the tensors

$$
\overleftrightarrow{v}_{1}:=\left(\begin{array}{lll}
v_{1, x x} & v_{1, x y} & v_{1, x z} \\
v_{1, y x} & v_{1, y y} & v_{1, y z} \\
v_{1, z x} & v_{1, z y} & v_{1, z z}
\end{array}\right), \overleftrightarrow{v}_{2}:=\left(\begin{array}{lll}
v_{2, x x} & v_{2, x y} & v_{2, x z} \\
v_{2, y x} & v_{2, y y} & v_{2, y z} \\
v_{2, z x} & v_{2, z y} & v_{2, z z}
\end{array}\right)
$$

respectively. Inserting these properties and the constitutive relation (3.1) into the definition of the magnetic voltage (3.11) yields

$$
\begin{aligned}
\widehat{h}_{x}(n) & =\int_{\widetilde{L}_{x}(n)}\left(v_{x x} B_{x}+v_{x y} B_{y}+v_{x z} B_{z}\right) \mathrm{d} x \\
& =\sum_{i=1}^{2} \int_{\widetilde{L}_{x}^{(i)}(n)}\left(v_{i, x x} B_{x}+v_{i, x y} B_{y}+v_{i, x z} B_{z}\right) \mathrm{d} x \\
& \approx B_{x} \bar{v}_{x x}(n) \widetilde{L}_{x}(n)+\sum_{i=1}^{2}\left(B_{i, y} v_{i, x y}+B_{i, z} v_{i, x z}\right) \widetilde{L}_{x}^{(i)}(n),
\end{aligned}
$$

where the integrals are approximated with an average value of the integrand times the length of the integration interval in the last transformation. Moreover, the definition of the averaged inverse permeability (3.25) has been employed for the $x$-component. In the next step the components of the magnetic inductance occurring in the last term of equation (3.94) have to be expressed in terms of magnetic fluxes $\widehat{b}$. As already shown in equation (3.23) for the construction of the diagonal permeability matrix in subsection 3.4.4, the $x$-component can be well approximated with

$$
B_{x} \approx \widehat{\bar{b}}_{x}(n) / A_{x}(n)
$$

For the $y$-and z-components, the situation is less obvious since these components are not available on the dual edge $\widetilde{L}_{x}(n)$. As suggested by [57, 60,61], the computationally most efficient way is to perform spatial interpolations of the two field components for the $y$ - and the $z$-direction available in each primary cell. Since the dual edge $\widetilde{L}_{x}(n)$ is allocated in the mid-

2 The quantities $M_{a}(a=x, y, z)$ indicate the number by which the global cell index $n$ (i.e. the position of the vector component of primary node- or dual node-like vectors) increases if one proceeds one cell in direction $a$. Note that this definition is thus slightly different compared to the one in section 3.4 .5 , where the positions of vectors with three components (i.e. $E$-field- or $H$-field-like vectors) were considered. Yet, the definition of $M_{a}$ for primary node- or dual node-like vectors both for ordering scheme 1 and 2 coincides with the one for $E$-fieldand $H$-field-like vectors for ordering scheme 1 (cf. Eq. (3.36) and (3.37)). For the ease of notation, $M_{a}$ is thus used without superscript in the following. 
dle of the facet $A_{x}(n)$, the interpolation effectively reduces to the calculation of the arithmetic mean:

$$
\begin{aligned}
& B_{1, y} \approx \frac{\widehat{\bar{b}}_{y}\left(n-M_{x}\right)+\widehat{\widehat{b}}_{y}\left(n-M_{x}+M_{y}\right)}{2 A_{y}\left(n-M_{x}\right)}, \\
& B_{1, z} \approx \frac{\widehat{\widehat{b}}_{z}\left(n-M_{x}\right)+\widehat{\widehat{b}}_{z}\left(n-M_{x}+M_{z}\right)}{2 A_{z}\left(n-M_{x}\right)}, \\
& B_{2, y} \approx \frac{\widehat{\bar{b}}_{y}(n)+\widehat{\widehat{b}}_{y}\left(n+M_{y}\right)}{2 A_{y}(n)}, \\
& B_{2, z} \approx \frac{\widehat{\widehat{b}}_{z}(n)+\widehat{\widehat{b}}_{z}\left(n+M_{z}\right)}{2 A_{z}(n)} .
\end{aligned}
$$

Combining equations (3.94), (3.95) and (3.96), performing analogous derivations for the $\widehat{h}_{y}(n)$ - and $\widehat{h}_{z}(n)$-components and taking into account the relations

$$
\widetilde{L}_{a}^{(j)}(i)=\frac{1}{2} L_{a}(j) \quad \text { (both for } i=j \text { and } i=j+M_{a} \text { ), }
$$

the desired relations which implicitly define the material matrix of the inverse permeability are obtained. In compact notation it takes the form $\left(1 \leq p, q \leq N_{\text {cells }}\right)$ :

$$
\left[\mathbf{M}_{\stackrel{\leftrightarrow}{\leftrightarrow}}\right]_{(a, p),(b, q)}=\left\{\begin{array}{cl}
\bar{v}_{a a}(p) \frac{\widetilde{L}_{a}(p)}{A_{a}(p)} & b=a ; q=p \\
v_{a b}\left(p-M_{a}\right) \frac{L_{a}\left(p-M_{a}\right)}{4 A_{b}\left(p-M_{a}\right)} & b \neq a ; q=p-M_{a} \vee q=p-M_{a}+M_{b} \\
v_{a b}(p) \frac{L_{a}(p)}{4 A_{b}(p)} & b \neq a ; q=p \vee q=p+M_{b} \\
0 & \text { else }
\end{array}\right\} .
$$

Note that there are two possibilities for $b \neq a$, that is to say for direction $b$ being different from direction $a$. Thus, the inverse permeability tensor has 9 nonzero entries in each row for indices associated with dual edges allocated completely inside gyromagnetic material. For taking into account zero entries due to boundary conditions and DOFs located outside the computation domain, $\mathbf{M}_{\leftrightarrow}$ is formally multiplied from the left and the right with $\mathbf{D}_{0}^{\mathrm{H}}$ (cf. section 3.4.7.1). Expression (3.98) holds in full generality also for dual edges located at material boundaries and regions with isotropic material for which off-diagonal terms of the inverse physical permeability tensor $v_{a b}$ vanish. The definition (3.98) then reduces to diagonal form as in the standard FIT (cf. Eq. (3.26)) inside regions with isotropic material.

Moreover, for the computation of eigenmodes of models containing gyromagnetic material, a Hermitian structure of the system matrix and consequently of the material matrices is of particular importance. As a non-Hermitian matrix has, in general, non-orthogonal eigenvectors, different algorithms have to be employed in such cases (cf. section 3.5). The condition for a Hermitian inverse permeability tensor reads (cf. Eq. (3.98))

$$
\left[\mathbf{M}_{\stackrel{\leftrightarrow}{v}}\right]_{(a, p),(b, q)} \stackrel{!}{=}\left(\left[\mathbf{M}_{\stackrel{v}{\leftrightarrow}}\right]_{(b, q),(a, p)}\right)^{*}
$$

where the complex conjugate is denoted by the asterisk $(*)$. This condition reduces to the form

$$
v_{a b}(p) \frac{L_{a}(p)}{4 A_{b}(p)} \stackrel{!}{=}\left(v_{b a}(p)\right)^{*} \frac{L_{b}(p)}{4 A_{a}(p)}
$$


for all tensor elements. Since

$$
A_{a}(n)=L_{b}(n) L_{c}(n) \text { with } a \neq b ; a \neq c ; b \neq c,
$$

it readily follows

$$
v_{a b}(p) \stackrel{!}{=}\left(v_{b a}(p)\right)^{*}
$$

This means that, provided that losses are absent and hence the physical local permeability tensor is Hermitian, $\mathbf{M}_{\stackrel{v}{\leftrightarrow}}$ is always Hermitian, even for non-equidistant grids. Some remarks are also due for the averaging of the off-diagonal elements for cells at the border of the computation domain or next to PEC-cells. In order to have a correct calculation of the average value of the magnetic field in such cells, the factor $1 / 2$ should be omitted in equations (3.96). However, this modification would, in general, spoil the Hermitian structure of the inverse permeability matrix. For this reason, the above definition should not be changed for the sake of stable simulations in the lossless case (cf. [57]).

\section{Implementation details}

The direct implementation of the row-oriented definition (3.98) is potentially inefficient because one has to access components of the inverse of the physical permeability tensor, $v_{a b}$, from different (primary) mesh cells for the assembly of each row of $\mathbf{M} \stackrel{\leftrightarrow}{v}$. This should particularly be avoided for a parallel computation where the values are distributed onto different computing nodes. Thus, the above definition is expressed in a mesh-cell oriented form. For each primary mesh cell with index $p$ that contains gyromagnetic material, the following elements of the inverse permeability matrix are set:

$$
\left.\begin{array}{l}
{\left[\mathbf{M}_{\stackrel{v}{v}}\right]_{(a, p),(a, p)}} \\
{\left[\mathbf{M}_{\stackrel{v}{v}}\right]_{\left(a, p+M_{a}\right),\left(a, p+M_{a}\right)}}
\end{array}\right\}+=v_{a a}(p) \frac{L_{a}(p)}{2 A_{a}(p)},
$$

Herein, the indices for the directions $a$ and $b$ loop over all possible combinations out of the domain $a, b \in\{x, y, z\}$ such that $b \neq a$. Since there are two possibilities of choosing $b \neq a$, in total 30 elements are set per mesh cell. The operator $+=$ for the first two elements in (3.103) means that the expression on the right hand side is to be added to the current value of the specified element (assuming that the element was initialized with zero). This way, the average value $\bar{v}_{a a}$ is calculated according to equation (3.25) implicitly. Note that also the main diagonal elements of cells filled with non-gyromagnetic materials which are located next to gyromagnetic cells have to be set for a correct averaging. Clearly, also with the implementation according to definition (3.103) elements have to be sent between different processes for the matrix assembly on a computer cluster. Yet, since the inverse of the physical permeability is evaluated taking into account the bias magnetic field and the current frequency in each primary cell, the evaluation of the tensor for the same cell on different processes is avoided. What is more, if the DOFs 


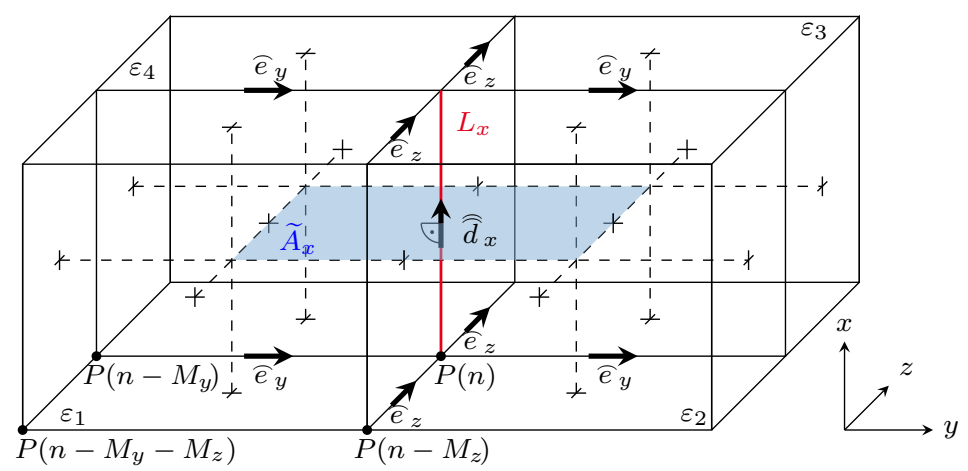

Figure 3.8.: Intersection of the primary edge $L_{x}$ with the dual facet $\widetilde{A}_{x}$. For the construction of the permittivity matrix, averaging of the permittivity on the dual facet is performed. [59]

of the FIT are ordered as in scheme 2 (cf. subsection 3.4.5.2), the number of sent messages can significantly be reduced. With this regard, it is advantageous to allow divisions of the vectors (and consequently matrices) to different processes only between blocks of $x$-, $y$ - and $z$-components with different cell indices. Thus, in total a smaller number of messages has to be sent compared to a direct implementation of the row-oriented definition (3.98).

\subsubsection{Modeling of gyroelectric materials}

For the derivation of the permittivity tensor for the FIT, the constitutive relation between the displacement field $\vec{D}$ and the electric field $\vec{E}$ for gyroelectric materials as given in equation (3.2) is considered. After transformation to FIT-notation, this relation takes the form

$$
\widehat{\widehat{d}}=\mathbf{M}_{\stackrel{\varepsilon}{\leftrightarrow}} \widehat{\mathbf{e}}
$$

where $\mathbf{M}_{\overleftrightarrow{\varepsilon}}$ is a non-diagonal permittivity matrix, whose explicit form is derived in the following. To begin with, the electric flux on the dual facet $\widetilde{A}_{x}(n)$ is considered, which is partially located in the four primary cells with primary node indices $P(n), P\left(n-M_{y}\right), P\left(n-M_{z}\right)$ and $P\left(n-M_{y}-M_{z}\right)$ (cf. Fig. 3.8). In the most general case, the dielectric properties inside each of these four cells can be described by permittivity tensors of the form

$$
\overleftrightarrow{\varepsilon}_{i}:=\left(\begin{array}{lll}
\varepsilon_{i, x x} & \varepsilon_{i, x y} & \varepsilon_{i, x z} \\
\varepsilon_{i, y x} & \varepsilon_{i, y y} & \varepsilon_{i, y z} \\
\varepsilon_{i, z x} & \varepsilon_{i, z y} & \varepsilon_{i, z z}
\end{array}\right) \text {, }
$$


where the index $i$ refers to one of the four cells. From the definition of the electric flux (cf. Eq. (3.8)) one then obtains

$$
\begin{aligned}
\widehat{\bar{d}}_{x}(n) & =\iint_{\widetilde{A}_{x}(n)}\left(\varepsilon_{x x} E_{x}+\varepsilon_{x y} E_{y}+\varepsilon_{x z} E_{z}\right) \mathrm{d} A \\
& =\sum_{i=1}^{4} \iint_{\widetilde{A}_{x}^{(i)}(n)}\left(\varepsilon_{i, x x} E_{x}+\varepsilon_{i, x y} E_{y}+\varepsilon_{i, x z} E_{z}\right) \mathrm{d} A \\
& \approx E_{x} \bar{\varepsilon}_{x x}(n) \widetilde{A}_{x}(n)+\sum_{i=1}^{4}\left(\varepsilon_{i, x y} E_{i, y}+\varepsilon_{i, x z} E_{i, z}\right) \widetilde{A}_{x}^{(i)}(n) .
\end{aligned}
$$

In the last transformation the integrands are approximated with the average value of the field component in this area times the integration area. Furthermore, the definition of the areaaveraged permittivity (cf. Eq. (3.29) in subsection 3.4.4) is inserted for the $x$-component. The remaining task is then to substitute the occurring components of the electric field with electric voltages $\widehat{e}$. Recalling the relation (3.27), $E_{x}$ is replaced with

$$
E_{x} \approx \frac{\widehat{e}_{x}(n)}{L_{x}(n)}
$$

However, the $y$ - and z-component of the electric field are not immediately available on $\widetilde{A}_{x}(n)$ and have to be approximated by spatial interpolation. Following again the minimal principle of $[57,60,61]$, these components are calculated by taking the arithmetic mean from the two primary edges closest to the partial dual areas, i.e.

$$
\begin{aligned}
& E_{1, y} \approx E_{4, y} \approx \frac{\widehat{e}_{y}\left(n-M_{y}\right)+\widehat{e}_{y}\left(n-M_{y}+M_{x}\right)}{2 L_{y}\left(n-M_{y}\right)} \\
& E_{2, y} \approx E_{3, y} \approx \frac{\widehat{e}_{y}(n)+\widehat{e}_{y}\left(n+M_{x}\right)}{2 L_{y}(n)} \\
& E_{1, z} \approx E_{2, z} \approx \frac{\widehat{e}_{z}\left(n-M_{z}\right)+\widehat{e}_{z}\left(n-M_{z}+M_{x}\right)}{2 L_{z}\left(n-M_{z}\right)} \\
& E_{3, z} \approx E_{4, z} \approx \frac{\widehat{e}_{z}(n)+\widehat{e}_{z}\left(n+M_{x}\right)}{2 L_{z}(n)}
\end{aligned}
$$

The explicit form of the permittivity tensor in the FIT is obtained by combining the last three relations (3.106), (3.107) and (3.108) and repeating the same procedure for the electric fluxes $\widehat{\vec{d}}_{y}(n)$ and $\widehat{d}_{z}(n)$. By taking into account the properties $(a=x, y, z ; b \neq a ; c \neq a ; c \neq b)$

$$
\widetilde{A}_{a}^{(i)}(j)=\frac{1}{4} L_{b}(i) L_{c}(i) \quad\left(\text { for } i=j, i=j-M_{b}, i=j-M_{c} \text { or } i=j-M_{b}-M_{c}\right),
$$

as well as

$$
L_{a}(n)=L_{a}\left(n \pm M_{b}\right),
$$


the expression can be simplified to $\left(1 \leq p, q \leq N_{\text {cells }} ; a=x, y, z ; c \neq a ; c \neq b\right)$

$$
\left[\mathbf{M}_{\stackrel{\varepsilon}{\varepsilon}}\right]_{(a, p),(b, q)}=\left\{\begin{array}{cl}
\bar{\varepsilon}_{a a}(p) \frac{\widetilde{A}_{a}(p)}{L_{a}(p)}, & b=a ; q=p \\
\left\{\begin{array}{c}
\frac{1}{8} \varepsilon_{a b}\left(p-\Delta_{b}-\Delta_{c}\right) L_{c}\left(p-\Delta_{c}\right) \\
+\frac{1}{8} \varepsilon_{a b}\left(p-\Delta_{b}\right) L_{c}(p)
\end{array}\right\}, & b \neq a ;\left\{\begin{array}{l}
q=p-\Delta_{b} \\
q=p-\Delta_{b}+\Delta_{a}
\end{array}\right\} \\
\left\{\begin{array}{l}
\frac{1}{8} \varepsilon_{a b}\left(p-\Delta_{c}\right) L_{c}\left(p-\Delta_{c}\right) \\
+\frac{1}{8} \varepsilon_{a b}(p) L_{c}(p)
\end{array}\right\}, & b \neq a ;\left\{\begin{array}{l}
q=p \\
q=p+\Delta_{a}
\end{array}\right\} \\
0, & \text { else }
\end{array}\right\}
$$

Since there are two ways of choosing the direction $b \neq a$ in the second and third row of the above definition, the permittivity tensor effectively has 9 nonzero entries per row inside the computation domain provided that none of the off-diagonal elements $\varepsilon_{a b}$ vanishes. Also note that this definition does not take into account additional zero entries due to boundary conditions or elements outside the computation domain. This is, however, readily accomplished by multiplying the permittivity matrix with the diagonal matrix $\mathbf{D}_{0}^{\mathrm{E}}$ from the left and the right (cf. section 3.4.7.1). Finally, for regions with fully isotropic material the definition (3.111) simplifies to the form of the standard FIT (cf. Eq. (3.30)). Moreover, definition (3.111) results in a manifestly Hermitian matrix for the lossless case of Hermitian physical local permittivity tensors. It is notable that the structure remains Hermitian for the non-equidistant grid. In order to retain this property, the averaging factors of off-diagonal elements should not be modified at cells with gyroelectric material at the border of the computation domain or PEC-cells (cf. subsection 3.4.8.1).

\section{Implementation details}

For the same reasons as mentioned for the inverse permeability tensor (cf. subsection 3.4.8.1), an implementation according to a mesh-cell oriented definition is preferable compared to the row-oriented definition (3.111) with regard to performance. For each primary mesh cell with index $p$ that is filled with gyroelectric material, the following elements of the permittivity matrix are set:

$$
\left.\begin{array}{l}
{\left[\mathbf{M}_{\overleftrightarrow{\varepsilon}}\right]_{(a, p),(a, p)}} \\
{\left[\mathbf{M}_{\leftrightarrow}\right]_{\left(a, p+M_{b}\right),\left(a, p+M_{b}\right)}} \\
{\left[\mathbf{M}_{\leftrightarrow}^{\leftrightarrow}\right]_{\left(a, p+M_{b}+M_{c}\right),\left(a, p+M_{b}+M_{c}\right)}}
\end{array}\right\}+=\varepsilon_{a a}(p) \frac{A_{a}(p)}{4 L_{a}(p)},
$$


The indices for the directions $a, b$ and $c$ loop over all possible combinations out of the domain $a, b, c \in\{x, y, z\}$ such that $b \neq a, c \neq a$ and $c \neq b$. Since there are two possibilities of choosing $b \neq a$, in total 60 elements are set per mesh cell (12 elements involving $\varepsilon_{a a}$ and 48 elements with $\varepsilon_{a b}$ ). The operator $+=$ in (3.112) denotes that the expression on the right hand side is added to the current value of the specified element (assuming that each element was initialized with zero). Thus, effectively an averaging of the permittivity for the main diagonal elements according to equation (3.25) is performed. This requires also that the main diagonal elements of cells filled with non-gyroelectric materials next to gyroelectric cells have to be set. Since four set values contribute for the averaging of the main diagonal matrix elements and similarly two set values for the averaging of the off-diagonal ones, the total of 60 set values is in accordance with the 9 nonzero entries per coordinate direction of the row-oriented definition (3.111).

\subsubsection{Curl-curl equation in the FIT}

Analogous to the derivation of the continuous curl-curl equation in section 3.1 , the one in FIT notation is obtained as follows. Differentiation of the Maxwell-Grid-Equation (3.16) with respect to time for time-harmonic fields, i.e. $\widehat{\mathbf{e}}(t)=\widehat{\mathbf{e}}_{n} \cdot e^{i \omega t}$, yields

$$
\widetilde{\mathbf{C}}\left(\frac{\mathrm{d}}{\mathrm{d} t} \widehat{\mathbf{h}}\right)=\frac{\mathrm{d}^{2}}{\mathrm{~d} t^{2}} \widehat{\mathbf{d}}=-\omega^{2} \widehat{\widehat{\mathbf{d}}} .
$$

Taking into account the constitutive relations (3.92) and (3.104) and inserting equation (3.113) into the Maxwell-Grid-Equation (3.15) readily leads to the curl-curl equation in FIT notation

$$
\mathbf{M}_{\overleftrightarrow{\varepsilon}}^{-1} \widetilde{\mathbf{C M}} \underset{\stackrel{\leftrightarrow}{\mathbf{C}}}{\mathbf{C}}=\omega^{2} \widehat{\mathbf{e}}
$$

Alternatively, the above equation can directly be obtained from the continuous one (3.5) by replacing the individual elements with the correspondent ones of the FIT.

If the material matrices $\mathbf{M}_{\overleftrightarrow{\varepsilon}}$ and $\mathbf{M}_{\overleftrightarrow{v}}$ are Hermitian, which includes also the special case of

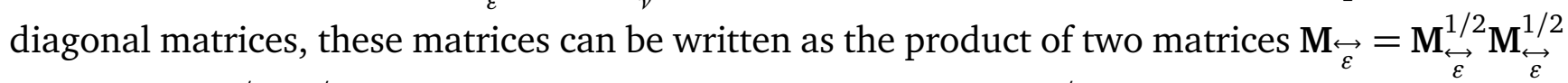
and $\mathbf{M}_{\stackrel{v}{s}}=\mathbf{M}_{\stackrel{v}{1 / 2}}^{\mathbf{M}_{\overleftrightarrow{v}}^{1 / 2}}$. By introducing the transformation $\widehat{\mathbf{e}}^{\prime}=\mathbf{M}_{\overleftrightarrow{\varepsilon}}^{1 / 2} \widehat{\mathbf{e}}$, it is thus possible to obtain the Hermitian version of the curl-curl equation [52]

$$
\mathbf{M}_{\overleftrightarrow{\varepsilon}}^{-1 / 2} \widetilde{\mathbf{C}} \mathbf{M}_{\stackrel{\leftrightarrow}{\leftrightarrow}} \mathbf{C M}_{\overleftrightarrow{\varepsilon}}^{-1 / 2} \widehat{\mathbf{e}}^{\prime}=\omega^{2} \widehat{\mathbf{e}}^{\prime}
$$

Since the above transformation may be interpreted as a similarity transformation, equation (3.115) features the same eigenvalues as the original equation (3.114). Yet, thanks to the manifestly Hermitian structure, specialized algorithms for the computation of the eigensystem may be employed.

\subsubsection{Lumped elements}

In general, lumped elements can be incorporated into the FIT by formally adding a supplementary current $\widehat{\mathbf{j}}_{L}$ due to the lumped element in Ampère's circuital law (3.16) [62, 63]:

$$
\widetilde{\mathbf{C}} \widehat{\mathbf{h}}=\frac{\mathrm{d}}{\mathrm{d} t} \widehat{\mathbf{d}}+\widehat{\mathbf{j}}+\widehat{\mathbf{j}}_{L}
$$




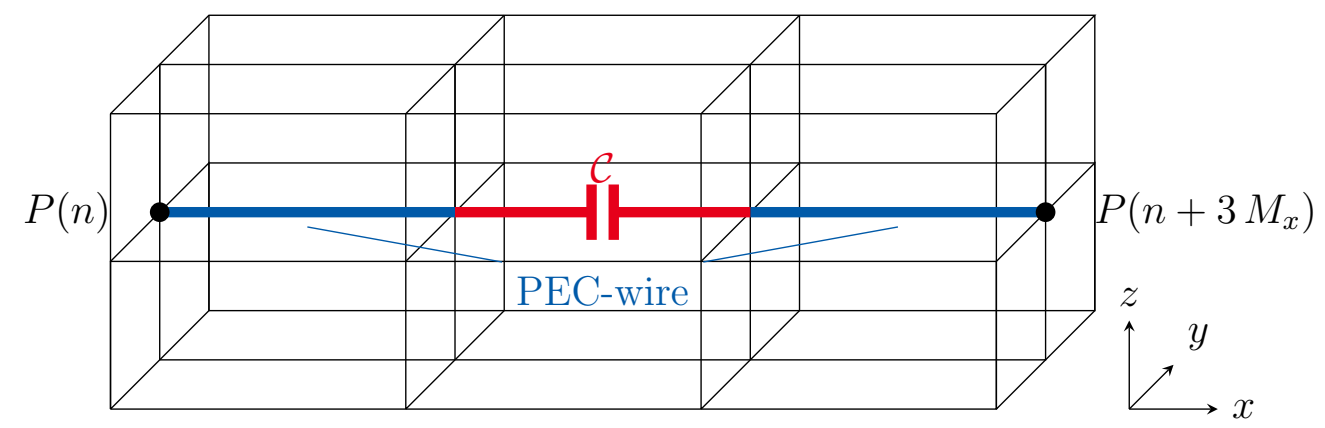

Figure 3.9.: Lumped element capacitor with capacitance $\mathscr{C}$ between the two primary nodes $P(n)$ and $P\left(n+3 M_{x}\right)$. Since the distance between these two nodes is larger than the length of one primary edge, the actual capacitance is allocated only on one edge (red) whereas the remaining edges are modeled as PEC-wires (blue).

The actual form of the current $\widehat{\hat{\mathbf{j}}}_{L}$ depends on the type of the lumped element. The two lumped elements relevant for this thesis, namely capacitors and resistors, are considered in more detail in the following for the case of time-harmonic electromagnetic fields, i.e. $\widehat{\mathbf{e}}(t)=\widehat{\mathbf{e}}_{n} \cdot e^{i \omega t}$.

The actual lumped element is supposed to be allocated only on one primary edge. If it is to be set between two nodes separated by a larger distance, a path connecting these two points is modeled as a PEC-wire while placing the actual lumped element only on one edge somewhere on that path (cf. Fig. 3.9).

\subsubsection{Capacitor}

The additional current $\widehat{\widehat{\mathbf{j}}}_{C}$ due to a purely capacitive lumped element with capacitance $\mathscr{C}_{n}$ allocated on the primary edge / dual facet with index $n$ can be expressed as

$$
\widehat{\hat{j}}_{C}=I_{n}=\mathscr{C}_{n} \frac{\mathrm{d}}{\mathrm{d} t} U_{n}(t)=\mathscr{C}_{n}(-\mathrm{i} \omega) \widehat{e}_{n},
$$

since the electric voltage $U_{n}$ along primary edge $n$ is given by (cf. Eq. (3.7))

$$
U_{n}(t)=\int_{L_{n}} \vec{E}(t) \cdot \mathrm{d} \vec{s}=: \widehat{\mathbf{e}}_{n}(t)=\widehat{e}_{n} \cdot e^{\mathrm{i} \omega t} .
$$

All lumped element capacitances $\mathscr{C}_{n}$ can conveniently be collected in one diagonal matrix $\mathbf{M}_{C}$ such that

$$
\mathscr{C}_{n} \widehat{e}_{n}=\left[\mathbf{M}_{C} \widehat{\mathbf{e}}\right]_{n}
$$

The overall effect of the lumped element capacitors is most easily seen in the modified curl-curl equation. Analogous to subsection 3.4.9 and with the definition (3.119), it is deduced as

$$
\left(\mathbf{M}_{\overleftrightarrow{\varepsilon}}+\mathbf{M}_{C}\right)^{-1} \widetilde{\mathbf{C}} \mathbf{M}_{\stackrel{v}{ }} \mathbf{C} \widehat{\mathbf{e}}=\omega^{2} \widehat{\mathbf{e}}
$$

Hence, the lumped element capacitance matrix $\mathbf{M}_{C}$ can simply be added to the original permittivity matrix.

Lumped element capacitors are used throughout this thesis for the simulation of the reflection measurement setup as well as to model the gap capacitance of the GSI SIS 18 cavity. 
Given a lumped element resistor with the Ohmic resistance $R_{n}=U_{n} / I_{n}$, which is allocated at the primary edge with index $n$, the components of the associated current $\widehat{\mathbf{j}}_{R}$ reads

$$
\widehat{\hat{j}}_{R}=I_{n}=\frac{\widehat{e}_{n}}{R_{n}} \text {. }
$$

Deriving yet another time the curl-curl equation including this additional current yields

$$
\mathbf{M}_{\stackrel{\varepsilon}{(}}^{-1} \widetilde{\mathbf{C}} \mathbf{M}_{\stackrel{\leftrightarrow}{\leftrightarrow}} \mathbf{C e}=\omega^{2} \widehat{\mathbf{e}}+\frac{i \omega}{R_{n}} \widehat{e}_{n} \mathbb{1}_{n}
$$

where $\mathbb{1}_{n}$ is an appropriate unit vector. This suggests to define the diagonal matrix $(1 \leq p \leq$ $\left.3 N_{\text {cells }}\right)$

$$
\left[\mathbf{M}_{R}\right]_{p}:=\left\{\begin{array}{cl}
\frac{\mathrm{i}}{\omega R_{n}} & p=n \\
0 & \text { else }
\end{array}\right\},
$$

which again can be added to the original permittivity matrix. The resistance matrix $\mathbf{M}_{R}$ is purely imaginary and frequency dependent. Thus, the incorporation of lumped element resistors leads to complex nonlinear eigenvalue problems, which can, however, be handled by the developed eigensolver.

Though one might want to model the resistance of, e.g., a tetrode with such a lumped element resistor in order to simulate the loaded cavity, this does not seem to be useful in practical applications. The reason is the complicated dependence of the resistive load on a variety of parameters such as frequency and RF-power level, for which no accurate data are available for the GSI SIS 18 cavity. For a comparison of measured loaded and simulated unloaded ones, a transformation of the values as done in [64] is recommended instead.

\subsubsection{Magnetostatic field problem}

As pointed out in section 3.1, the inverse permeability tensor enters in the system matrix of the eigenvalue problem if gyromagnetic material is present and similarly the inverse permittivity tensor for gyroelectric material. Since the components of both tensors depend on the static bias magnetic field (cf. subsection 3.4.8), the bias field has to be determined by solving the magnetostatic field problem beforehand. To begin with, this problem is formulated in FITnotation in subsection 3.4.11.1. For its solution, the so-called $H_{i}$-algorithm is employed, which is explained in subsection 3.4.11.2 including its extension for nonlinear materials in subsection 3.4.11.3. Finally, a couple of test problems is considered for verification in subsection 3.4.11.4.

The following theoretical basics are a review of [52]. Yet, the description of the $H_{i}$-algorithm goes much more into the details as required for an actual implementation.

\subsubsection{Statement of the problem}

The magnetostatic field problem is governed by the two Maxwell's equations (3.16) and (3.18) for vanishing time-derivatives (in FIT-notation) [52]

$$
\begin{aligned}
\widetilde{\mathbf{C}} \widehat{\mathbf{h}}=\widehat{\widehat{\mathbf{j}}}, \\
\widehat{\mathbf{S} \widehat{\mathbf{b}}}=0,
\end{aligned}
$$


together with the constitutive relation (3.33). The current density $\widehat{\hat{\mathbf{j}}}$ in equation (3.124) contains all the steady currents such as ones for magnetic biasing. Note that even if gyrotropic materials are present, the diagonal inverse (chord) permeability matrix $\mathbf{M}_{v}$ instead of the tensorial expression of subsection 3.4.8.1 enters in equation (3.33) since the tensor describes rather the dynamic response of the system than its static behavior. Nonetheless, the nonlinear characteristics of the ferrite has to be taken into account.

Usually, the above stated field problem is solved by introducing a vector potential $\widehat{a}$ such that $\widehat{\mathbf{b}}=$ Ca $[4,52]$. This ansatz manifestly fulfills equation (3.125) and leads to the remaining equation in $\widehat{\mathbf{a}}$

$$
\widetilde{\mathbf{C}} \mathbf{M}_{v} \mathbf{C a}=\widehat{\mathbf{j}} .
$$

Unfortunately, the system matrix of the above equation is of dimension $\left(3 N_{\text {cells }}\right) \times\left(3 N_{\text {cells }}\right)$ (for the non-reduced grid) and thus computationally comparatively expensive to solve. As shown in [52], it is, however, possible to formulate the problem in a way such that the dimension reduces to only $N_{\text {cells }} \times N_{\text {cells }}$ by employing the so-called $H_{i}$-algorithm, which is reviewed in the following subsection.

\subsubsection{2 $H_{i}$-algorithm}

The $H_{i}$-algorithm [52] is based on the Helmholtz decomposition. According to this fundamental theorem [65], the magnetic field can be resolved into the sum of two fields

$$
\vec{H}=\vec{H}_{i}+\vec{H}_{h}
$$

with the solenoidal field $\vec{H}_{i}$

$$
\nabla \times \vec{H}_{i}=\vec{J}
$$

and the irrotational field $\vec{H}_{h}$, which can be expressed in terms of a scalar potential $\varphi$

$$
\vec{H}_{h}=-\nabla \varphi
$$

In FIT-notation this decomposition takes the form

$$
\begin{array}{rlrl}
\widehat{\widehat{\mathbf{h}}} & =\widehat{\mathbf{h}}_{i}+\widehat{\mathbf{h}}_{h} \\
\text { with } & \widetilde{\mathbf{C}}_{\mathbf{h}_{i}} & =\widehat{\hat{\mathbf{j}}} \\
\text { and } & \widehat{\mathbf{h}}_{h} & =-\widetilde{\mathbf{G}} \Phi,
\end{array}
$$

where the scalar potential $\Phi$ is allocated on dual nodes. The $H_{i}$-algorithm hence starts with the construction of the field $\widehat{\mathbf{h}}_{i}$. As this vector field may be chosen arbitrarily and may, in particular, be unphysical, a solution as simple as possible should be preferred. To begin with, the special case in which no currents enter or leave the computation domain is considered. Hence, there are only closed current paths inside the computation domain. The following algorithm can then be used (after [52]): 


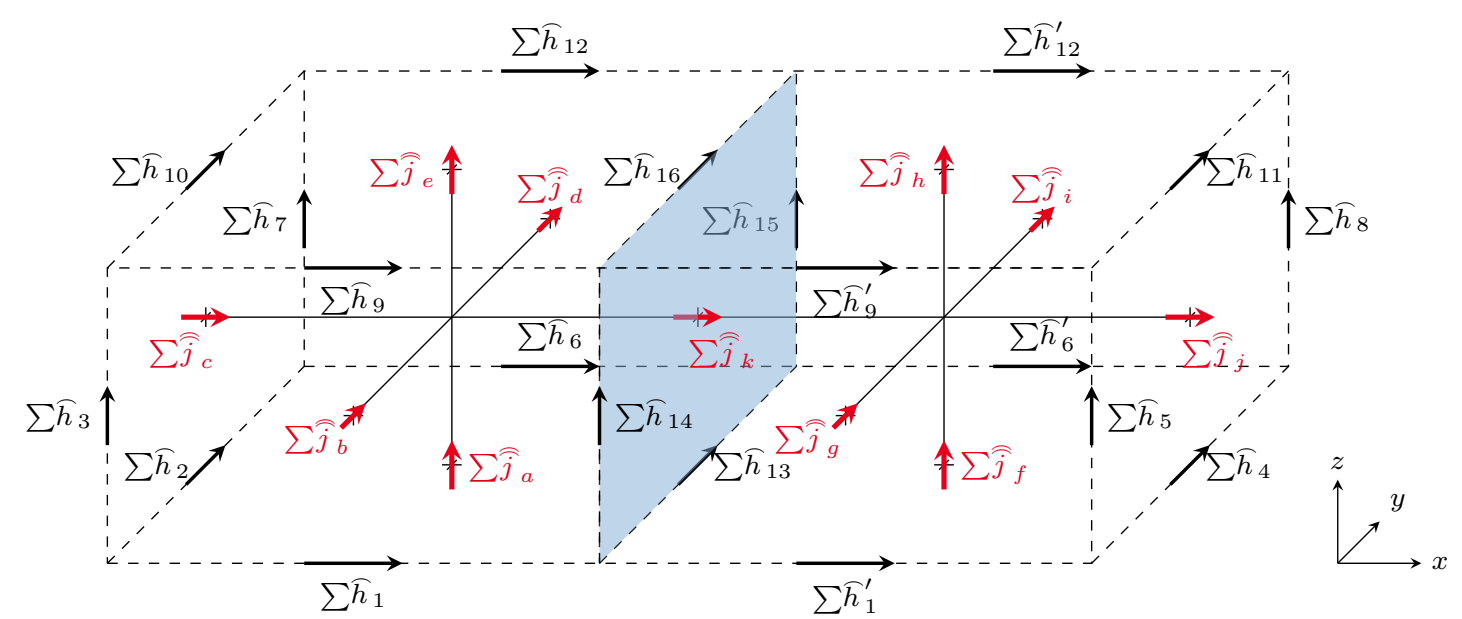

Figure 3.10.: Subdivision of a subvolume in $x$-direction at the middle facet. For the determination of the magnetic field on the four edges 13-16, only the four side surfaces on the left (facets $a, b, d, e)$ are considered. The sum of all $\widehat{h}_{i}$-components along a (dual) edge of the subvolume is denoted by $\sum \widehat{h}$, whereas the sum of all currents through a (dual) surface is denoted by $\sum \widehat{\hat{j}}$.

1. All $\widehat{\mathbf{h}}_{i}$-components are initialized with 0 .

2. The smallest possible subvolume that contains all current paths is determined. Since no current runs through the surface of the subvolume, the rotational equation (3.130b) is fulfilled trivially on the surface. The case that currents penetrate through the surface is treated separately below.

3. The subvolume is divided into two subvolumes of approximately equal size. The $\widehat{\mathbf{h}}_{i}$ components have to be set on the four new edges of the interface of the subdivision according to equation (3.130b). This is done as follows: The four side surfaces of which one edge coincides with one of the four new edges are considered consecutively. By convention only those side surfaces (of the two possible choices) are selected that have the smaller facet indices (cf. Fig. 3.10). For each of these four side surfaces, the $\widehat{\mathbf{h}}_{i}$-components are already known along three of their (surface) edges. Consequently, the sum of all $\widehat{\mathbf{h}}_{i}$ components along the fourth (surface) edge can be determined according to equation (3.130b). The hereby obtained value for the sum of all $\widehat{\mathbf{h}}_{i}$-components along the fourth (surface) edge is distributed equally on all dual edges along the fourth surface edge. Possibly different cell sizes or edge lengths are not taken into account at this point. The proof that, following this procedure, the rotational equation (3.130b) is indeed satisfied on all new side surfaces can be found in the appendix A.2.2.

4. Each of the new subvolumes is successively further subdivided until all $\widehat{\mathbf{h}}_{i}$-components are known. To avoid round-off errors, the direction of subdivisions should by altered cyclically [52].

For the more general case of a current path going through the surface of the computation domain, step 2 of the algorithm has to be modified. To begin with, the initial subvolume is set to the whole volume of the computation domain. One possible solution to initialize the $\widehat{\mathbf{h}}_{i}$ - 


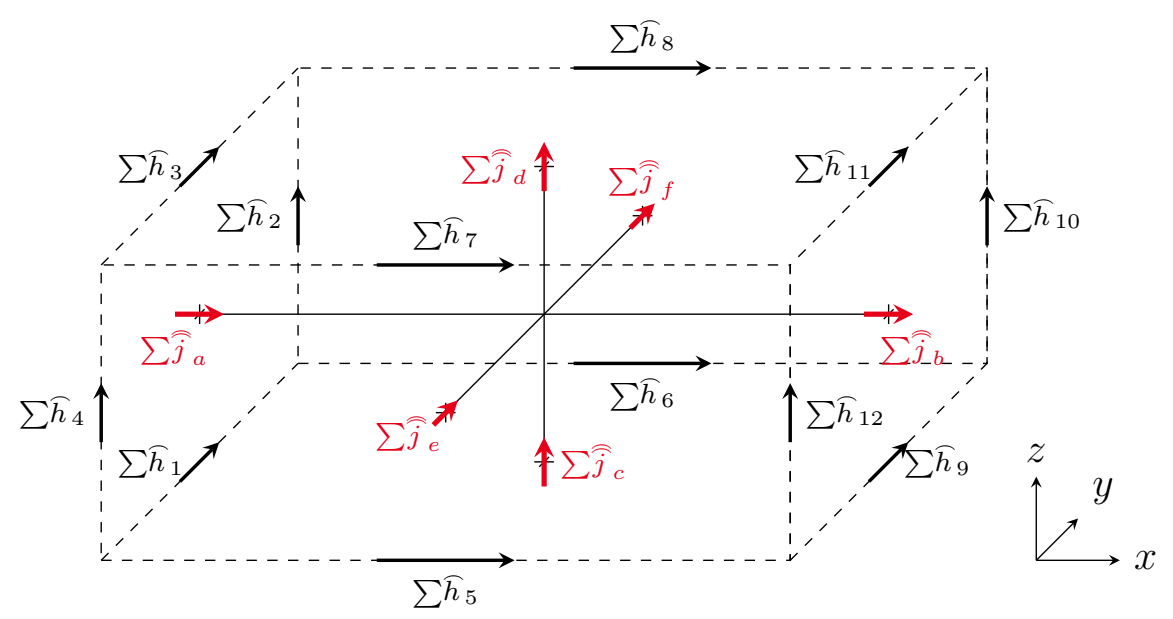

Figure 3.11.: Notation used for the determination of $\widehat{\mathbf{h}}_{i}$-components on the edges of the initial subvolume. The sum of all $\widehat{h}_{i}$-components along a (dual) edge of the subvolume is denoted by $\sum \widehat{h}$, whereas the sum of all currents through a (dual) surface is denoted by $\sum \widehat{\hat{j}}$.

components on the edges of the surface, which has been derived as part of this thesis, is (for notation see Fig. 3.11):

$$
\begin{aligned}
& \sum \widehat{h}_{1}=\frac{1}{4}\left(\sum \widehat{\hat{j}}_{b}-\sum \widehat{\hat{j}}_{d}\right)-\frac{1}{3} \widehat{\hat{j}}_{\Delta y} \\
& \sum \widehat{h}_{2}=\frac{1}{4}\left(\sum \widehat{\hat{j}}_{b}+\sum \widehat{\hat{j}}_{f}\right)-\frac{1}{3} \widehat{\hat{j}}_{\Delta y}-\frac{1}{3} \widehat{\hat{j}}_{\Delta z}, \\
& \sum \widehat{h}_{3}=\frac{1}{4}\left(-\sum \widehat{\hat{j}}_{b}-\sum \widehat{\hat{j}}_{d}\right)+\frac{1}{3} \widehat{\hat{j}}_{\Delta y}+\frac{1}{3} \widehat{\hat{j}}_{\Delta z}, \\
& \sum \widehat{h}_{4}=\frac{1}{4}\left(-\sum \widehat{\hat{j}}_{b}+\sum \widehat{\hat{j}}_{f}\right)+\frac{1}{3} \widehat{\hat{j}}_{\Delta z}, \\
& \sum \widehat{h}_{5}=\frac{1}{4}\left(\sum \widehat{\hat{j}}_{d}-\sum \widehat{\hat{j}}_{f}\right)-\frac{1}{3} \widehat{\hat{j}}_{\Delta y}+\frac{1}{3} \widehat{\hat{j}}_{\Delta z}, \\
& \sum \widehat{h}_{6}=\frac{1}{4}\left(-\sum \widehat{\hat{j}}_{d}-\sum \widehat{\hat{j}}_{f}\right)-\frac{1}{3} \widehat{\hat{j}}_{\Delta z} \text {, } \\
& \sum \widehat{h}_{7}=\frac{1}{4}\left(\sum \widehat{\hat{j}}_{d}+\sum \widehat{\hat{j}}_{f}\right)+\frac{1}{3} \widehat{\hat{j}}_{\Delta y} \\
& \sum \widehat{h}_{8}=\frac{1}{4}\left(-\sum \widehat{\hat{j}}_{d}+\sum \widehat{\hat{j}}_{f}\right) \text {, } \\
& \sum \widehat{h}_{9}=\frac{1}{4}\left(\sum \widehat{\hat{j}}_{d}+\sum \widehat{\hat{j}}_{b}\right)+\frac{1}{3} \widehat{\hat{j}}_{\Delta z} \text {, } \\
& \sum \widehat{h}_{10}=\frac{1}{4}\left(\sum \widehat{\hat{j}}_{b}-\sum \widehat{\hat{j}}_{f}\right)-\frac{1}{3} \widehat{\hat{j}}_{\Delta y} \text {, } \\
& \sum \widehat{h}_{11}=\frac{1}{4}\left(\sum \widehat{\hat{j}}_{d}-\sum \widehat{\hat{j}}_{b}\right)+\frac{1}{3} \widehat{\hat{j}}_{\Delta z} \text {, } \\
& \sum \widehat{h}_{12}=\frac{1}{4}\left(-\sum \widehat{\hat{j}}_{b}-\sum \widehat{\hat{j}}_{f}\right)-\frac{1}{3} \widehat{\hat{j}}_{\Delta y} \text {. }
\end{aligned}
$$


Therein, the definitions

$$
\begin{aligned}
& \widehat{\hat{j}}_{\Delta y}:=\sum \widehat{\hat{j}}_{e}-\sum \widehat{\hat{j}}_{f} \\
& \widehat{\hat{j}}_{\Delta z}:=\sum \widehat{\hat{j}}_{c}-\sum \widehat{\hat{j}}_{d}
\end{aligned}
$$

are used. Moreover, the symbol $\sum$ refers to the sum of all $\widehat{\mathbf{h}}_{i}$-components along the specified edge or the sum of all currents through the specified surface, respectively. Obviously, if there are no currents through the surface, all expressions in (3.131) vanish in accordance with the special case mentioned above. For the derivation of (3.131) and the proof that these expressions satisfy the rotational equation (3.130b) on the surface is referred to subsection A.2.1 of the appendix.

After having determined the rotational field $\widehat{\mathbf{h}}_{i}$, the scalar potential is computed by solving equation (3.125), which for the ansatz (3.130) takes the form

$$
\text { with } \quad \begin{aligned}
\mathbf{S M}_{\mu} \mathbf{S}^{T} \boldsymbol{\Phi} & =\mathbf{q}_{\mathrm{m}} \\
\mathbf{q}_{\mathrm{m}} & :=-\mathbf{S M}_{\mu} \widehat{\mathbf{h}}_{i}
\end{aligned}
$$

and the (diagonal) permeability matrix $\mathbf{M}_{\mu}=\mathbf{M}_{v}^{-1}$. In equation (3.133a), the relation (3.76) between the topological matrices has been employed to emphasize the positive (semi-)definite structure of the system matrix. In fact, the matrix is positive semidefinite only for the nonreduced grid but positive definite for the reduced grid (cf. subsection 3.4.7). Thanks to this feature, efficient standard algorithms can be used for the solution of equation (3.133). Nevertheless, this is still the most expensive part for the computation of the magnetostatic field solution. In comparison, the time spent for the calculation of the $\widehat{\mathbf{h}}_{i}$-field with the above algorithm is essentially negligible.

As known from literature [52], the $H_{i}$-algorithm may suffer from loss of significance when high-permeable materials are present. On modern systems with double-precision floating-point format, cancellations do, however, not occur for realistic problems. Nevertheless, to be able to solve even models with extremely high permeabilities without difficulties, the new implementation of the algorithm supports also an iterative $H_{i}$-update-method (similar to [52]). If $n$-fold refinement is activated, the magnetostatic problem is solved at first for a material distribution with a relative permeability of only $\mu_{r}^{1 / 2^{n}}$ of the original value $\mu_{r}$. In each of the $2^{n}$ iterations, the permeability is then increased by the factor $\mu_{r}^{1 / 2^{n}}$. This way, the calculation of the magnetic field at the boundary between vacuum and a material with $\mu_{r}=10^{20}$ could be performed accurately for testing purposes.

\subsubsection{Extension for nonlinear material}

For materials with nonlinear material characteristics, such as ferrites, equation (3.133) exhibits a nonlinear character since the permeability itself depends on the magnetic field strength. In this work, simple successive substitution [66] is applied until convergence of the value for the permeability is observed (for the conditions on the $B-H$ curve for the existence of a unique solution see, e.g., [67]). That is to say, the magnetostatic problem is solved for some initial homogeneous distribution of the permeability at first. Then the updated values for the permeability are calculated according to the nonlinear characteristic equation and the current magnetic field distribution. This is repeated iteratively until the relative change of the permeability between two 


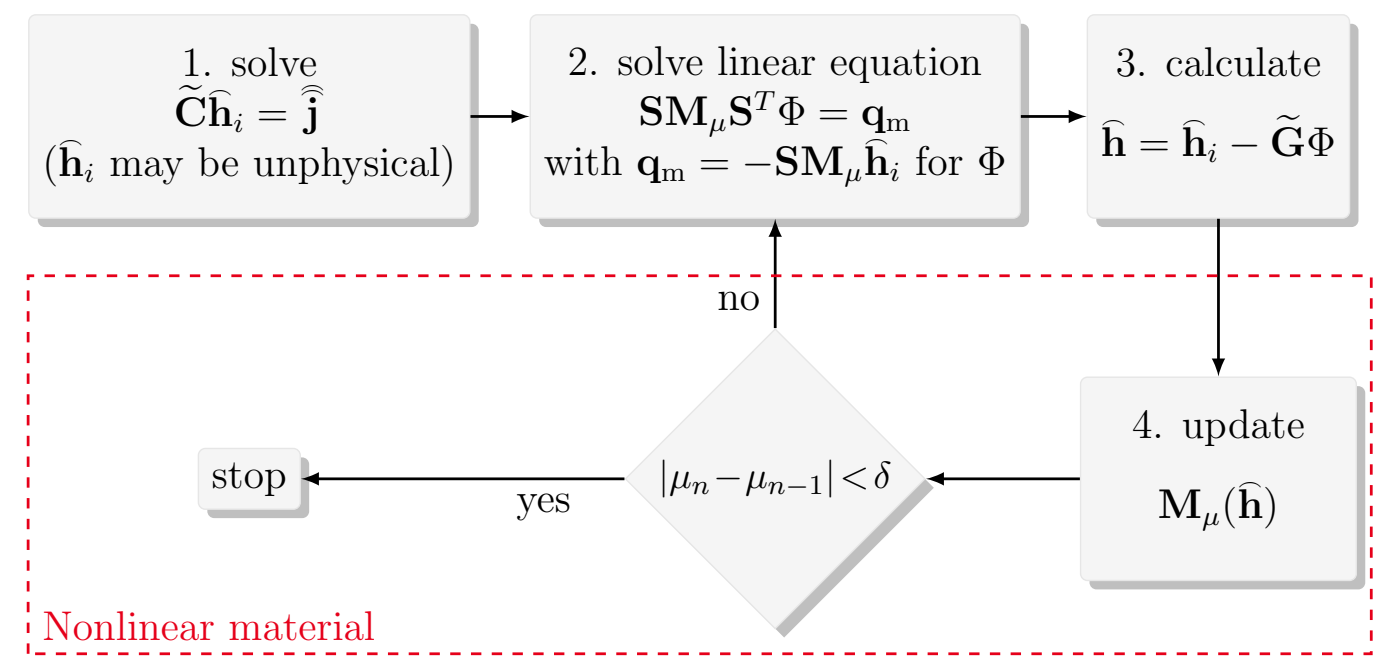

Figure 3.12.: Simplified flowchart of the $H_{i}$-algorithm. The solenoidal field $\widehat{\mathbf{h}}_{i}$, which satisfies the rotational equation ( $3.130 \mathrm{~b})$, is constructed at first (step 1). After that, the linear equation (3.133), which originates from the divergence equation (3.125), is solved for the scalar potential $\Phi$ (step 2). This allows to calculate the composed magnetic field $\widehat{h}$ (step 3). For nonlinear material characteristics, equation is (3.133) is nonlinear. Hence, it is solved for some initial permeability in the beginning. Taking into account the obtained field distribution this way in step 3, the permeability matrix is updated (step 4). The iterative solution process continues until the maximal relative change of the permeability values in all mesh cells drops below the specified threshold $\delta$.

subsequent iterations drops below a specified threshold $\delta$ in all mesh cells (cf. also Fig. 3.12). Since the field solution and permeability values change only slightly in subsequent iterations, the preconditioner used for the solution of equation (3.133) can be kept for several steps. This reduces the total computation time drastically in practice.

Applying the Newton method to the solution of equation (3.133) instead could increase the convergence of the nonlinear iteration up to second order in the vicinity of the solution. The diagonal chord permittivity matrix on the left hand side of that equation would then have to be replaced with the non-diagonal differential permeability matrix [68]. Yet, since the solution of the magnetostatic field problem takes much less computation time than the subsequent computation of the eigenmodes anyway, the application of the Newton method is not further elaborated in this thesis. Besides, though the convergence rate of the successive substitution approach is usually significantly inferior compared to the Newton method, the former converges stably whereas the latter can easily suffer from instabilities [66].

For the update of the permeability matrix in each nonlinear iteration, the knowledge of the magnetic field inside every primary cell that contains nonlinear material is required. As the magnetic field components are allocated on dual edges in the FIT (cf. subsection 3.4.2), averaging of the magnetic field is required, which is discussed in the following. 


\section{Averaging of the magnetic field strength}

To begin with, the following notation is introduced. Let a magnetic voltage with additional superscript ( $i$ ) refer only to that part which is allocated (on the partial dual edge) in the primary cell with index $i$. For instance,

$$
\widehat{h}_{a}^{(i)}(n)=\int_{\widetilde{L}_{a}^{(i)}(n)} \vec{H} \cdot \mathrm{d} \vec{a}=: h_{a}^{(i)}(n) \widetilde{L}_{a}^{(i)}(n) \quad(a=x, y, z),
$$

where the mean magnetic field component on that partial dual edge $h_{a}^{(i)}(n)$ is defined by the above equation (cf. also Fig. 3.2(b)). Accordingly, the mean value for the magnetic field strength $\bar{H}(i)$ in the primary cell with index $i$ is calculated as

$$
\bar{H}(i)=\frac{1}{2} \sqrt{\left(h_{x}^{(i)}(i)+h_{x}^{(i)}\left(i+M_{x}\right)\right)^{2}+\left(h_{y}^{(i)}(i)+h_{y}^{(i)}\left(i+M_{y}\right)\right)^{2}+\left(h_{z}^{(i)}(i)+h_{z}^{(i)}\left(i+M_{z}\right)\right)^{2}} .
$$

It is thus the remaining task to determine the quantities $h_{a}^{(i)}(i)$ and $h_{a}^{(i)}\left(i+M_{a}\right)$ as follows [69]. Decomposing the magnetic voltage

$$
\widehat{h}_{a}(i)=\widehat{h}_{a}^{\left(i-M_{a}\right)}(i)+\widehat{h}_{a}^{(i)}(i)=h_{a}^{\left(i-M_{a}\right)}(i) \widetilde{L}_{a}^{\left(i-M_{a}\right)}(i)+h_{a}^{(i)}(i) \widetilde{L}_{a}^{(i)}(i)
$$

and employing the condition of continuity for the magnetic field

$$
h_{a}^{\left(i-M_{a}\right)}(i) \mu\left(i-M_{a}\right)=h_{a}^{(i)}(i) \mu(i)
$$

yields by inserting equation (3.137) into equation (3.136)

$$
h_{a}^{(i)}(i)=\frac{\widehat{h}_{a}(i)}{\frac{\mu(i)}{\mu\left(i-M_{a}\right)} \widetilde{L}_{a}^{\left(i-M_{a}\right)}(i)+\widetilde{L}_{a}^{(i)}(i)}=\frac{2 \widehat{h}_{a}(i)}{\frac{\mu(i)}{\mu\left(i-M_{a}\right)} L_{a}\left(i-M_{a}\right)+L_{a}(i)},
$$

where the relation (3.97) has been used. In case that the dual edge with index $i$ is located at the edge of the computation region towards small indices with respect to the orientation $a$, the expression simplifies to

$$
h_{a}^{(i)}(i)=h_{a}(i)=\frac{\widehat{h}_{a}(i)}{\widetilde{L}_{a}(i)} \quad \text { (at the edge of the computation region). }
$$

Analogously, one yields

$$
h_{a}^{(i)}\left(i+M_{a}\right)=\frac{\widehat{h}_{a}\left(i+M_{a}\right)}{\frac{\mu(i)}{\mu\left(i+M_{a}\right)} \widetilde{L}_{a}^{\left(i+M_{a}\right)}\left(i+M_{a}\right)+\widetilde{L}_{a}^{(i)}\left(i+M_{a}\right)}=\frac{2 \widehat{h}_{a}\left(i+M_{a}\right)}{\frac{\mu(i)}{\mu\left(i+M_{a}\right)} L_{a}\left(i+M_{a}\right)+L_{a}(i)} .
$$

Again, in the case that the dual edge with index $i$ is located at the edge of the computation region towards large indices with respect to the orientation $a$, this expression reduces to

$$
h_{a}^{(i)}\left(i+M_{a}\right)=h_{a}\left(i+M_{a}\right)=\frac{\widehat{h}_{a}\left(i+M_{a}\right)}{\widetilde{L}_{a}\left(i+M_{a}\right)} \quad \text { (at the edge of the computation region). }
$$

Having obtained the mean value of the magnetic field and hereby also immediately the permeability values inside each cell with nonlinear material, the updated inverse permeability matrix in the FIT can readily be calculated as explained in section 3.4.4. 
(a)

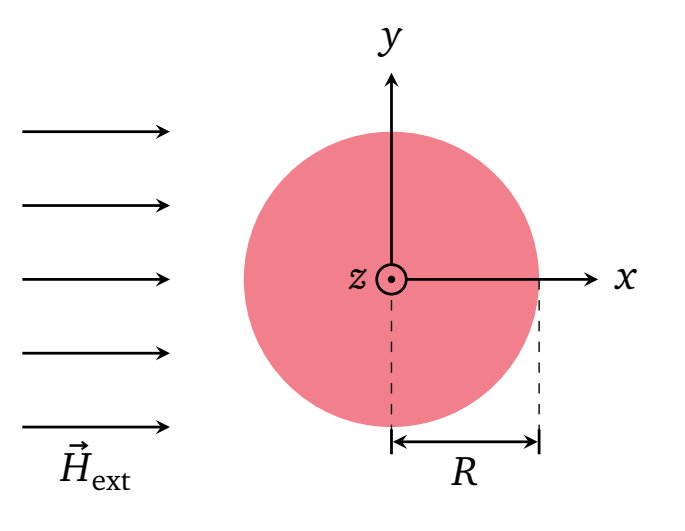

(b)

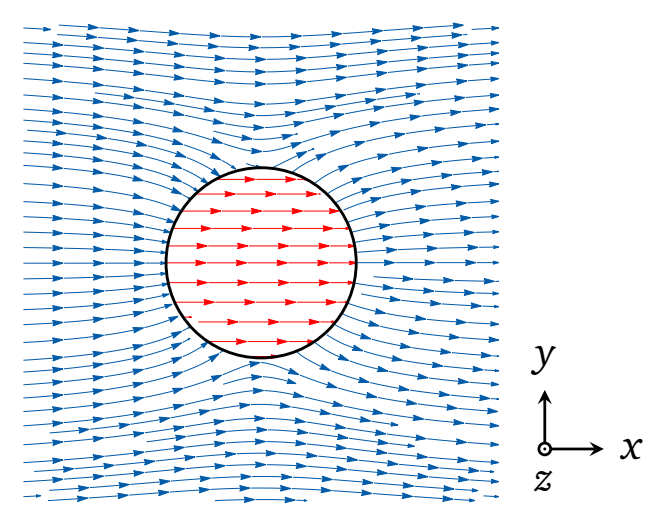

Figure 3.13.: (a) Cylinder with permeability $\mu_{r}$ subject to a homogeneous magnetic field $\vec{H}_{\text {ext }}=$ $H_{0} \cdot \vec{e}_{x}$. The cylinder axis is oriented in z-direction. (b) Field lines of the resulting magnetic field inside (red) and outside (blue) the cylinder for $\mu_{r}=5$ according to Eq. (3.142).

\subsubsection{Verification}

The correct implementation of the construction of the $H_{i}$-field (cf. subsection 3.4.11.2) can readily be verified by performing the $H_{i}$-algorithm for any given current distribution and thereafter checking the validity of the rotational equation (3.130b). For all tested current distributions, this condition is found to be fulfilled up to machine precision. Hence, it remains only to verify the solution of the divergence equation (3.133). To this end, the test model also considered in [70] is picked up. It consists of an infinitesimally long cylinder of radius $R$ and relative permeability $\mu_{r}$ with its axis oriented in z-direction and located in an external homogeneous magnetic field of strength $\vec{H}_{\text {ext }}=H_{0} \cdot \vec{e}_{x}$ (cf. Fig. 3.13(a)). The analytical solution for the resulting magnetic field $\vec{H}_{\text {res }}$ is given by:

$$
\begin{array}{ll}
\vec{H}_{\mathrm{res}}=H_{0} \frac{2}{\mu_{r}+1} \vec{e}_{x} & \text { for } r \leq R, \\
\vec{H}_{\mathrm{res}}=H_{0} \cdot \vec{e}_{x}+H_{0} \frac{R^{2}}{\left(x^{2}+y^{2}\right)^{2}} \times \frac{\mu_{r}-1}{\mu_{r}+1}\left(\left(x^{2}-y^{2}\right) \vec{e}_{x}+2 x y \cdot \vec{e}_{y}\right) & \text { for } r>R .
\end{array}
$$

The solution exhibits thus a homogeneous field parallel to the external field inside the cylinder (cf. Fig. 3.13(b)). To begin with, a cylinder filling with a linear material characteristic with $\mu_{r}=$ 10 and an external field strength of $H_{0}=10^{6} \mathrm{~A} / \mathrm{m}$ is assumed. According to equation (3.142a), this results in a field strength of $\left|\vec{H}_{\text {res }}\right|=181818 \mathrm{~A} / \mathrm{m}$ inside the cylinder. With those parameters for $\mu_{r}$ and $H_{0}$, the magnetostatic field problem is also solved with the new implementation of the solver described before. Electric boundary conditions are imposed in the $y$-and z-direction to enforce purely tangential magnetic field components at those boundaries, which allows for a discretization in z-direction with only one effective mesh cell. Moreover, surrounding free space with a distance of $19 \times R$ is added in lower and upper $x$ - and $y$-direction to reduce undesired effects due to the limited discretization domain. For the spatial discretization, the Perfect Boundary Approximation ${ }^{\circledR}$ (PBA) [48] is used for the linear material. To estimate the accuracy of the numerical solution, the energy norm of the difference, defined as [52]

$$
\left|\widehat{\mathbf{h}}_{\text {num }}-\widehat{\mathbf{h}}_{\text {analy }}\right|^{2}=\left(\widehat{\mathbf{h}}_{\text {num }}-\widehat{\mathbf{h}}_{\text {analy }}\right)^{T} \mathbf{M}_{\mu}\left(\widehat{\mathbf{h}}_{\text {num }}-\widehat{\mathbf{h}}_{\text {analy }}\right),
$$




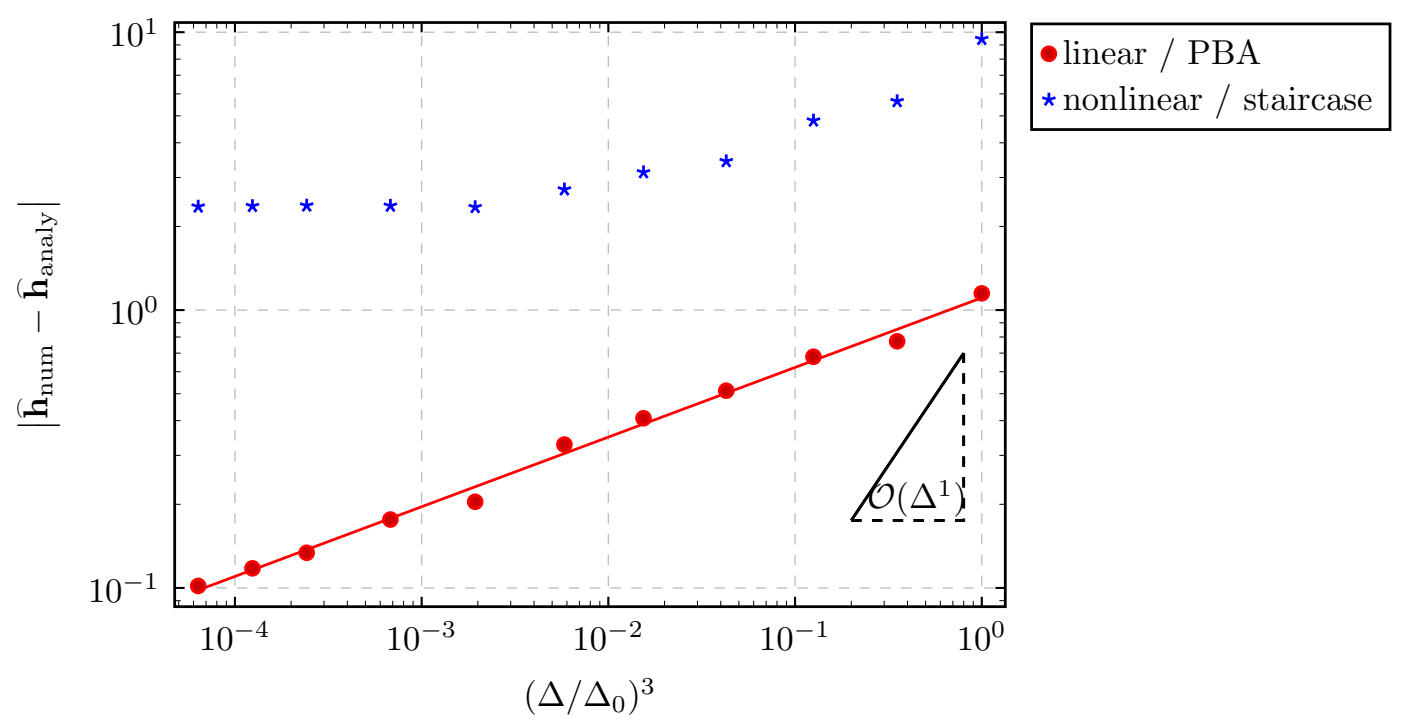

Figure 3.14.: Energy norm $\left|\widehat{\mathrm{h}}_{\text {num }}-\widehat{\mathrm{h}}_{\text {analy }}\right|$ of the difference between the numerically obtained magnetic field $\widehat{\mathbf{h}}_{\text {num }}$ and the analytical reference $\widehat{\mathbf{h}}_{\text {analy }}$ calculated on all dual edges that are located completely inside a cylinder with radius $0.8 \times R$ as a function of the normalized step size $\Delta / \Delta_{0}$ for the cylinder experiencing an external homogeneous magnetic field of strength $H_{0}=10^{6} \mathrm{~A} / \mathrm{m}$. The values are shown for the cylinder with linear material $\mu_{r}=10$ and a spatial discretization using PBA as well as for the cylinder filled with nonlinear iron material and a staircase filling.

where $\widehat{\mathbf{h}}_{\text {num }}$ and $\widehat{\mathbf{h}}_{\text {analy }}$ are the magnetic voltages of the numerical and analytical solution, respectively, is calculated on all dual edges that are located completely inside a cylinder with radius $0.8 \times R$. This is repeated for different mesh resolutions. As shown in figure 3.14, the numerical field solution converges towards the analytical one. Furthermore, the same test model is also treated for a cylinder filled with nonlinear iron material. The measurement data for pure iron can be found in $[48,70]$. Those data points are interpolated employing the method of "straight $B H$-lines" discussed in [70]. As the permeability in equation (3.142a) now depends on the magnetic field strength, the semi-analytical reference values are given by the roots of that equation as $\left|\vec{H}_{\text {res }}\right|=142976 \mathrm{~A} / \mathrm{m}$ and $\mu_{r}=12.9883$ inside the cylinder. Also the simulation is performed with iron filling. However, since a PBA-mesh is not supported by the implemented solver due to the averaging of the magnetic field strength and the construction of the permeability matrix, staircase filling is used for the nonlinear case. The obtained deviation from the reference values in terms of the energy norm are supplemented in figure 3.14. Obviously, stagnation is observed below a particular threshold of the mesh resolution, which depends on the radius inside which the field solutions are compared. In fact, local deviations from the analytical values at the surface of the cylinder even raise with decreasing step size of the mesh. This problem is intrinsic to the attempt of approximating a curved geometry with a staircase mesh and is known from literature $[67,70]$.

For the independent verification of the nonlinear algorithm, the following minimalist, effective one-dimensional test model is thus investigated. It consists of two (primary) mesh cells (cf. Fig. 3.15): the first cell of length $l_{1}$ contains a linear material with relative permeability $\mu_{1}$, the second one has length $l_{2}$ and is filled with a material with the nonlinear permeability $\mu_{2}(H)$. 


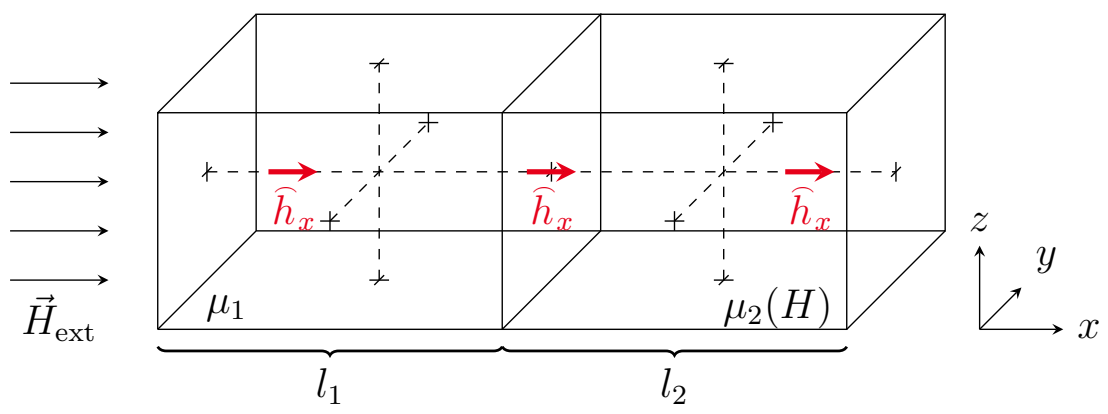

Figure 3.15.: Model for the verification of the nonlinear algorithm of the magnetostatic solver. The cell on the left of length $l_{1}$ contains a linear material with relative permeability $\mu_{1}$, the one on the right has length $l_{2}$ and is filled with a material with the nonlinear permeability $\mu_{2}(H)$. The cells are subject to a homogeneous magnetic field $\vec{H}_{\text {ext }}=$ $H_{0} \cdot \vec{e}_{x}$. Since the model is effectively one-dimensional, only the $\widehat{h}_{x}$-components are calculated.

Moreover, the cells are subject to an external homogeneous magnetic field of strength $H_{0}$ normal to the interface of the two cells. The same boundary conditions as for the cylinder are imposed. The analytical solution for the resulting magnetic field strengths $H_{1}$ and $H_{2}$ in the first and the second cell, respectively, for this setup takes the form:

$$
\begin{aligned}
& H_{1}=\frac{\mu_{2}\left(H_{2}\right)}{\mu_{1}} H_{2}, \\
& H_{2}=\frac{l_{1}+l_{2}}{l_{1} \mu_{2}\left(H_{2}\right) / \mu_{1}+l_{2}} H_{0} .
\end{aligned}
$$

If this field problem is solved numerically employing the averaging of the magnetic field strength as explained in subsection 3.4.11.3, there is no error due to spatial discretization at all regardless of the number of mesh cells, even for a non-equidistant grid. That is to say, the only contribution to the uncertainty in the numerical solution is due to the iterative solution of the nonlinear characteristic equation. This is confirmed by numerical studies using different length ratios $l_{1} /$ $l_{2}$ as well as models for the nonlinear permeability, including, e.g., iron using the interpolation method "straight $\mathrm{BH}$-lines". It is notable that the error due to spatial discretization is identically zero only because the condition of continuity of the magnetic field is taken into account for the averaging of the magnetic field (cf. subsection 3.4.11.3).

\subsection{Numerical methods for the computation of eigenvectors}

\subsubsection{General remarks}

The eigenvalue problem for a square matrix $A$ is defined by the equation

$$
A \vec{v}=\lambda \vec{v},
$$

where $\vec{v}$ with norm $\|\vec{v}\| \neq 0$ is called an eigenvector to the associated eigenvalue $\lambda$. The eigenvalues of $A$ are the roots of the characteristic equation

$$
\operatorname{det}(A-\lambda \mathbb{1})=0,
$$


whereas the eigenvectors are the nontrivial solutions of

$$
(A-\lambda \mathbb{1}) \vec{v}=0 \text {. }
$$

Since according to the Abel-Ruffini theorem [71] polynomial equations with degree five or higher do not have general algebraic solutions, numerical approaches have to be employed in practice to solve eigenvalue problems. A variety of numerical methods have been developed. Which one is best-suited for a particular problem depends on many criteria like the number of wanted eigenvectors, the location of the eigenvalues in the spectrum, the dimension of the system matrix or whether or not also associated eigenvectors are desired. Among the algorithms for small dense matrices are the power iteration method, the inverse power iteration method, and the Rayleigh quotient iteration. Since some knowledge about these single vector iterations is useful for a better understanding of more sophisticated approaches for large sparse systems, they are briefly introduced in the next section. After that, a deflation technique is treated in subsection 3.5.3 followed by some remarks on the QR algorithm in subsection 3.5.4. In subsection 3.5.5 fundamental principles related to the concept of subspace iterations are discussed. Finally, relevant details about the actual implementation are highlighted and verification examples are presented.

\subsubsection{Single vector iterations}

\subsubsection{Power method}

The simplest method to compute an eigenpair associated with the eigenvalue of largest modulus is the power iteration method [72]. The algorithm is as follows:

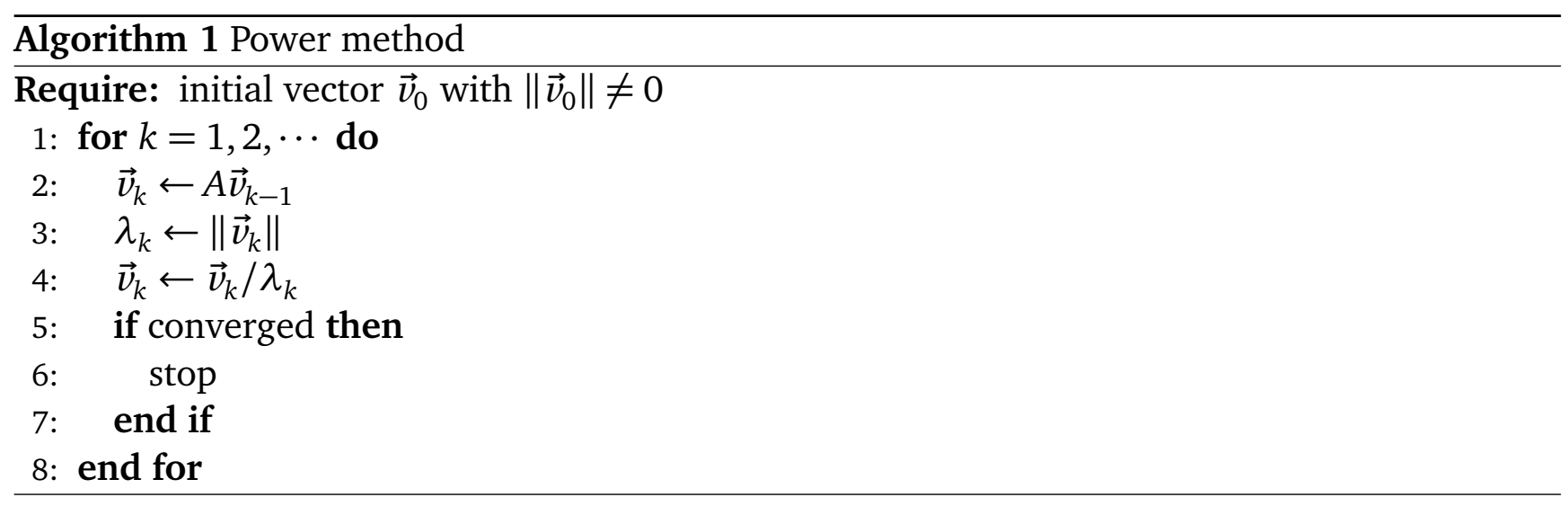

On convergence, $\vec{v}_{k}$ is an eigenvector of $A$ associated with the (dominant) eigenvalue $\lambda_{k}$. The power method is known to converge linearly with convergence factor $\left|\lambda_{\text {subdominant }}\right| /\left|\lambda_{\text {dominant }}\right|$ [72], where $\lambda_{\text {dominant }}$ and $\lambda_{\text {subdominant }}$ are the eigenvalues with largest and second largest modulus, respectively.

\subsubsection{Inverse iteration}

If one eigenvector associated with an eigenvalue inside the spectrum near some target value $\sigma$ is desired, instead of $A$ the matrix $(A-\sigma \mathbb{1})^{-1}$ can be used to iterate, which results in the algorithm: 


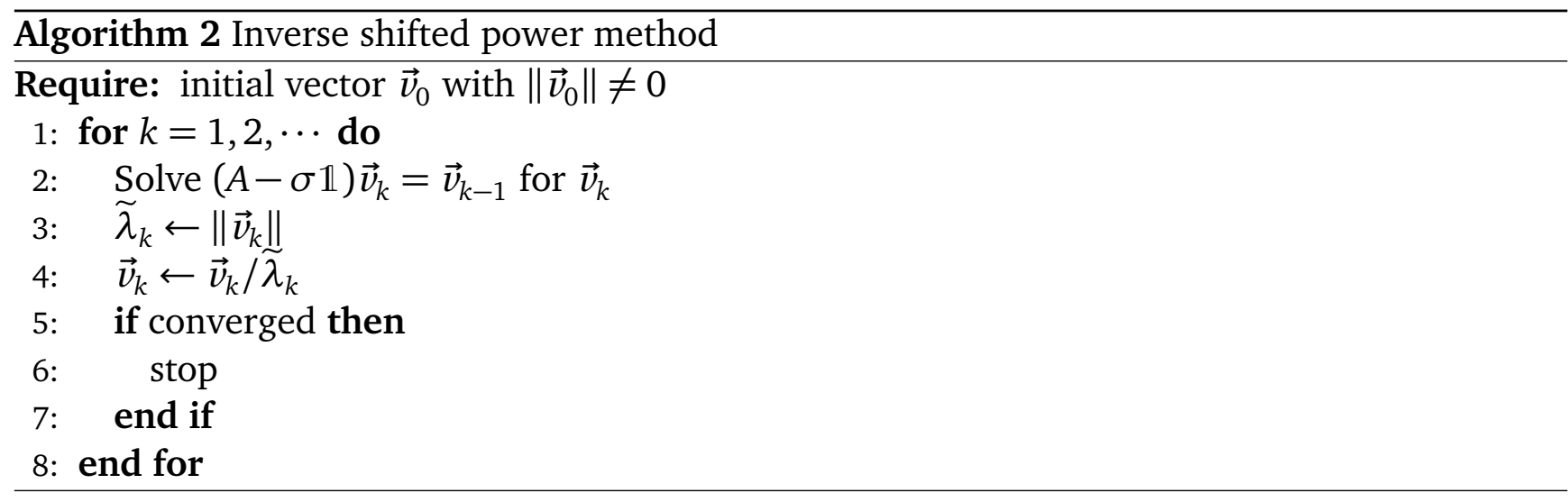

On convergence, $\vec{\nu}_{k}$ is an eigenvector of $A$ associated with the eigenvalue $\lambda_{k}=1 / \tilde{\lambda}_{k}+\sigma$. Since the inverse iteration is effectively a power method, linear convergence with rate $\mid \lambda_{\text {closest }}-$ $\sigma|/| \lambda_{2 \text { ndclosest }}-\sigma \mid$ is observed [72], where $\lambda_{\text {closest }}$ is the closest eigenvalue to $\sigma$ and $\lambda_{2 \text { ndclosest }}$ the next closest one.

\subsubsection{Rayleigh quotient iteration}

The Rayleigh quotient iteration may be regarded as an extension of the inverse iteration. Whereas in the latter the shift $\sigma$ remains unchanged throughout all iterations, here the Rayleigh quotient, defined as

$$
R Q=\frac{\vec{v}^{*} A \vec{v}}{\vec{v}^{*} \vec{v}}
$$

where $\vec{v}$ is an approximation of an eigenvector of $A$, is taken as the new shift in the following iteration. The algorithm thus takes the form:

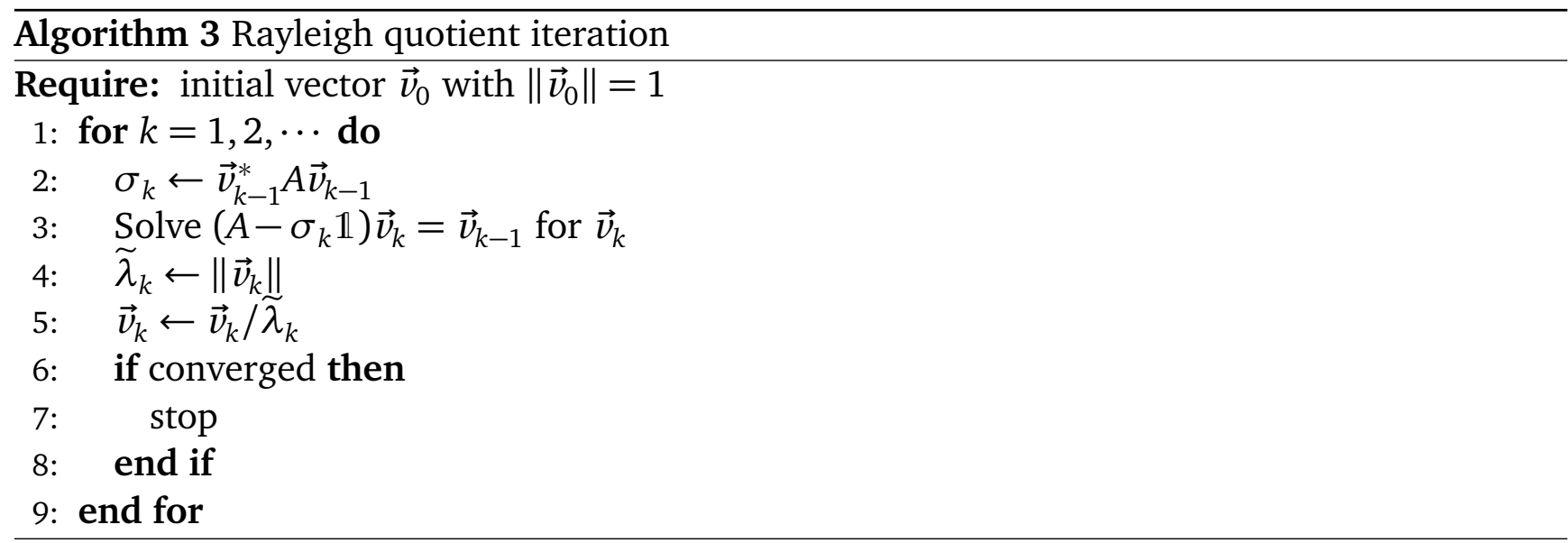

Though the Rayleigh quotient iteration features quadratic convergence and even cubic convergence for Hermitian matrices (provided it converges at all) [73], it suffers from the high cost for the solution of the equation in line 3 with a different shift in each iteration.

\subsubsection{Deflation}

The methods introduced in the previous section deliver only one eigenpair on convergence. For the computation of additional ones, components of the already obtained eigenvectors have to be 
removed by deflation techniques. One useful deflation method for Hermitian matrices is briefly introduced in the following subsection, the non-Hermitian case is addressed afterwards.

\subsubsection{Deflation for Hermitian eigenvalue problems}

Since it is possible to find an orthogonal basis for eigenvectors of Hermitian matrices, the matrix $A^{\perp}$, the restriction of the matrix $A$ to the invariant subspace orthogonal to the subspace spanned by the already obtained eigenvectors $Q=\operatorname{span}\left(\vec{v}_{i}\right)$, can be used for the computation of further eigenvalues [73]. The deflated matrix then takes the form [74]

$$
A^{\perp}=\left(\mathbb{1}-Q Q^{*}\right) A\left(\mathbb{1}-Q Q^{*}\right)
$$

\subsubsection{Deflation for non-Hermitian eigenvalue problems}

In contrast, for non-Hermitian matrices this deflation scheme is not immediately suitable due to the non-orthogonality of the eigenvectors. However, if the partial Schur-composition is available, the orthonormal Schur-vectors associated with the eigenvalues computed so far are useful for the construction of the deflated matrix according to equation (3.149) [74]. The relation between the Schur-form and the eigenpairs will be covered in more detail when discussing the $\mathrm{QR}$ algorithm in the following section.

\subsubsection{QR algorithm}

For the simultaneous computation of all eigenvalues of a matrix, the QR algorithm is particularly well suited. Based on the QR factorization, a sequence of similarity transformations of the system matrix $A$ is produced, which (under certain conditions [75]) converges to its Schurform. The basic algorithm is given by [73]:

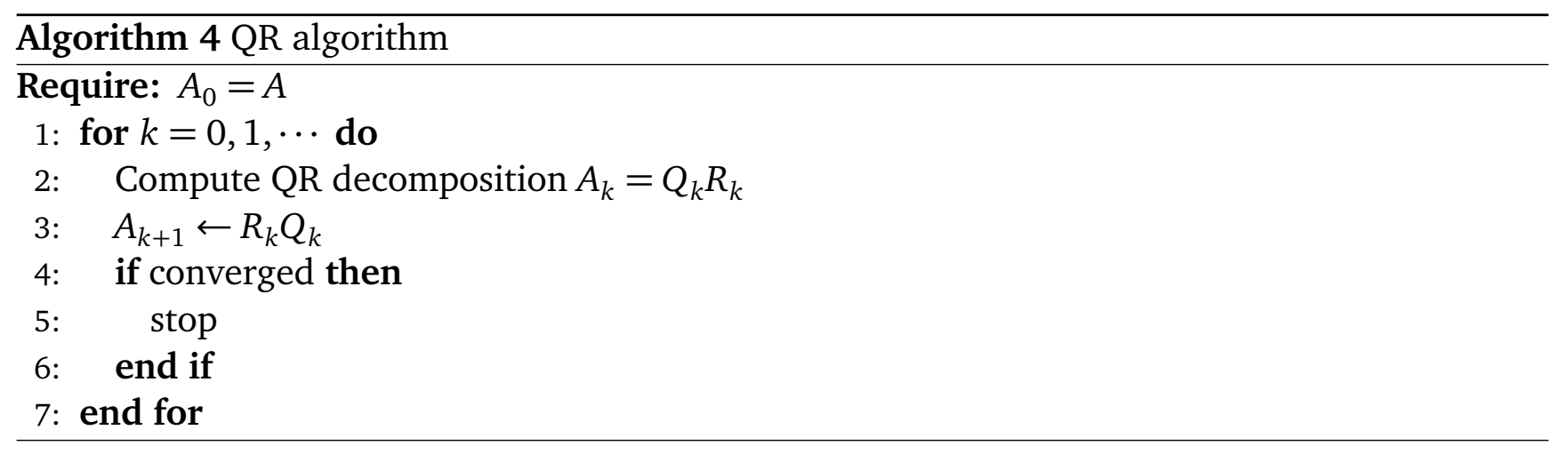

On convergence, matrix $A_{k}$ approaches the Schur-form of $A$, i.e. $A_{k}=\widetilde{Q}_{k}^{*} A \widetilde{Q}_{k}=U T U^{*}$, where $\widetilde{Q}_{k}=\prod_{i=0}^{k-1} Q_{i}, U$ is a unitary matrix and $T$ an upper triangular matrix. The eigenvalues of $A$ are found on the diagonal of $T$ since the updated matrix $A_{k+1}=R_{k} Q_{k}=Q_{k}^{*} Q_{k} R_{k} Q_{k}=Q_{k}^{*} A_{k} Q_{k}$ is in fact obtained by a similarity transformation out of the original matrix $A$. Moreover, if $A$ is a normal matrix ${ }^{3}, T$ is a diagonal matrix and the columns of $U$ are the eigenvectors of $A$. Yet,

3 A complex square matrix is normal if $A^{*} A=A A^{*}$ [76]. Particularly, a Hermitian matrix is normal. 
in the general case of a non-normal matrix only the eigenvector associated with the eigenvalue on the top left position of $T$, entry $T_{1,1}$, is immediately available as the first column of $U$. If desired, the remaining eigenvectors can be obtained one after another by reordering the Schurform such that the eigenvalue of the wanted eigenpair appears on the top left position of $T$ [77]. Due to its close relation to the power iteration, the QR algorithm features (at least) linear convergence of the eigenvalues provided that all the eigenvalues have different modulus [78]. The convergence can significantly be accelerated by applying spectral transformations [78].

Variants of the QR algorithm are for example implemented in the simple driver LAPACKsubroutines with endings -EV and -ES [79].

\subsubsection{Subspace methods with projection}

Some of the most successful methods for the extraction of eigenvalues of large sparse systems are based on the principle of subspace iteration. Its main characteristics are the projection onto a small system using a set of vectors spanning a subspace, extracting best possible approximations of the eigensolutions in that subspace and modifying the subspace in some manner with the aim of obtaining better approximations in the next iteration. A variety of different methods have been evolved, which can be categorized by the distinct approaches for the subspace extraction, that is, how the approximate eigensolutions are obtained from the subspace, and the subspace expansion. Regarding the first aspect, the Rayleigh-Ritz method and the harmonic Ritz method are introduced in subsection 3.5.5.1. After that, two of the most important classes of subspaces, the Krylov subspaces and those used in the Jacobi-Davidson method, are discussed in subsection 3.5.5.2.

\subsubsection{Extracting eigensolutions from subspaces}

This subsection addresses the computation of an approximation of an eigenpair of a system matrix $A$ from a given subspace $\mathscr{V}=\operatorname{span}\left(\vec{v}_{1}, \vec{v}_{2}, \cdots, \vec{v}_{m}\right)$, which is spanned by the orthonormal columns $\vec{v}_{i}(i=1,2, \cdots, m)$ of a matrix $V$. Different methods are available to serve this purpose. Which one is favorable depends mostly on the location of the desired eigenvalues in the spectrum. Whereas Ritz values are well suited for exterior eigenvalues, the usage of harmonic Ritz values is preferable for the interior ones. Some aspects about the two approaches are discussed in the following two paragraphs.

\section{The Rayleigh-Ritz method}

For the computation of extremal eigenvalues, the well-known Rayleigh-Ritz approach [51, 80, 81] is recommendable. Imposing the Galerkin-condition

$$
A V \vec{s}-\theta V \vec{s} \perp \mathscr{V}
$$

one obtains the projected eigenvalue problem

$$
V^{*} A V \vec{s}-\theta \vec{s}=0 .
$$

with the $m$ eigenpairs $\left(\theta_{i}, \vec{s}_{i}\right)$ as its solutions. Then $\theta_{i}$ and $\vec{y}_{i}=V \vec{s}_{i}$ can be taken as approximations of eigenvalues and eigenvectors of $A$ and are referred to as Ritz values and Ritz vectors, respectively, in literature. They have the following properties: 
- The Ritz values $\theta_{i}$ are optimal approximations of the eigenvalues of $A$ in the given subspace [73].

- The Ritz interval $\left[\theta_{i}-\left\|\vec{r}_{i}\right\|, \theta_{i}+\left\|\vec{r}_{i}\right\|\right]$ with the residual vector $\vec{r}_{i}=A \vec{y}_{i}-\vec{y}_{i} \theta_{i}$ contains an eigenvalue of $A$ [73]. Note, however, that overlapping intervals may contain the same eigenvalue [73].

- The full set of all Ritz pairs $\left\{\left(\theta_{i}, \vec{y}_{i}\right)\right\}$ provide the best possible approximations to the eigenpairs of $A$ which can be obtained from the subspace $\mathscr{V}$ alone [73]. Yet, in general, one single Ritz vector alone is not the best approximation of any of the eigenvectors of $A$ in $\mathscr{V}$ for $\operatorname{dim} \mathscr{V}>1$ [73]. In fact, the error bound $\|A \vec{y}-\vec{y} \theta\| /\|\vec{y}\|$ is not minimized by any of the Ritz vectors (for $\operatorname{dim} \mathscr{V}>1$ ) [73].

- There is no error bound on Ritz vectors [73]. Nonetheless, if the residual norm $\left\|\vec{r}_{i}\right\|$ approaches zero, convergence of the Ritz vector to an eigenvector is ensured [81].

\section{The harmonic Ritz method}

The standard Rayleigh-Ritz method as previously introduced may yield disappointing results for the eigenvalues located in the interior of the spectrum as the Ritz values tend to converge to exterior eigenvalues $[74,82]$. Hence, if one wants to compute interior eigenvalues close to a target $\tau$, the Harmonic Ritz method [83] might be favorable. Here the Galerkin-condition

$$
(A-\tau \mathbb{1}) V \vec{s}-\theta V \vec{s} \perp \mathscr{W}
$$

is imposed, where the subspace $\mathscr{W}$ is chosen as $\mathscr{W}:=(A-\tau \mathbb{1}) \mathscr{V}$ and is spanned by the matrix $W=(A-\tau \mathbb{1}) V$. This condition leads to the generalized eigenvalue problem

$$
V^{*}(A-\tau \mathbb{1})^{*}(A-\tau \mathbb{1}) V \vec{s}=(\theta-\tau) V^{*}(A-\tau \mathbb{1})^{*} V \vec{s} .
$$

By requiring $W$ to be an orthonormal system, it can be reduced to the standard eigenvalue problem [74]

$$
W^{*}(A-\tau \mathbb{1})^{-1} W \vec{s}=(\theta-\tau)^{-1} \vec{s} .
$$

The $m$ eigensolutions $\left(\theta_{i}, \vec{s}_{i}\right)$ are referred to as harmonic Ritz values and harmonic Ritz vectors, respectively. The obtained equation (3.154) can be interpreted as an orthogonally projected eigenvalue problem for the matrix $(A-\tau \mathbb{1})^{-1}$ (cf. Eq. (3.151)) [74]. Thus, the harmonic Ritz values are identified as Ritz values for $(A-\tau \mathbb{1})^{-1}$, which (under certain conditions, see [84]) converge towards eigenvalues of $A$ near the target $\tau$. Note also that it is not necessary to explicitly invert the matrix $(A-\tau \mathbb{1})$ as one can compute the system matrix of the small projected problem from the right hand side of equation (3.153) provided that the orthogonal transformations performed on $W$ are also applied to $V$ in order to maintain the relation $W=(A-\tau \mathbb{1}) V$ [74].

\subsubsection{Expansion of subspaces}

Obviously, the quality of the approximation depends on the vectors spanning the subspace $\mathscr{V}$. One can only expect to be able to extract accurate approximations of an eigenvector if the subspace contains strong components in the direction of this eigenvector. Consequently, the aim is to expand the subspace such a way that these components are amplified. Two important methods are introduced in the following. 


\section{Krylov subspace}

The Krylov subspace is defined as [72]

$$
\mathscr{K}_{m}(A, \vec{v})=\left\{\vec{v}, A \vec{v}, A^{2} \vec{v}, \cdots, A^{m-1} \vec{v}\right\} .
$$

That is, the subspace is spanned by powers of the system matrix acting on a starting vector $\vec{v}$.

\section{Jacobi-Davidson subspace}

Alternatively, the subspace can be expanded by the solution $\vec{t}$ of a correction equation, which is discussed in the following. Suppose $\vec{u}$ is a given approximation of an eigenvector of the system matrix $A$ with associated eigenvalue $\lambda$. Following the idea of Jacobi [85], we want to find a correction $\vec{t}$ to this approximation that is orthogonal to $\vec{u}$ and satisfies

$$
A(\vec{u}+\vec{t})=\lambda(\vec{u}+\vec{t}) .
$$

As we are only interested in corrections $\vec{t} \perp \vec{u}$, the matrix $A$ can be replaced by its orthogonal projection onto the subspace orthogonal to $\vec{u}$, which takes the form

$$
A^{\perp}=\left(\mathbb{1}-\vec{u} \vec{u}^{*}\right) A\left(\mathbb{1}-\vec{u} \vec{u}^{*}\right) .
$$

Moreover, since the exact eigenvalue $\lambda$ is usually not available in practice, it is obviously substituted with its currently best approximation $\theta$. Then, equation (3.156) can be written as

$$
\left(\mathbb{1}-\vec{u} \vec{u}^{*}\right)(A-\theta \mathbb{1})\left(\mathbb{1}-\vec{u} \vec{u}^{*}\right) \vec{t}=\vec{r},
$$

with the residual

$$
\vec{r} \equiv(A-\theta \mathbb{1}) \vec{u}
$$

Equation (3.158) is known as the Jacobi-Davidson correction equation.

\subsubsection{The Arnoldi and Lanczos algorithms}

Arnoldi and Lanczos type iterations combine the Rayleigh-Ritz method (cf. subsection 3.5.5.1) with the construction of an orthonormal basis of the sequence of Krylov subspaces (cf. subsection 3.5.5.2). The Arnoldi procedure is as follows [72]:

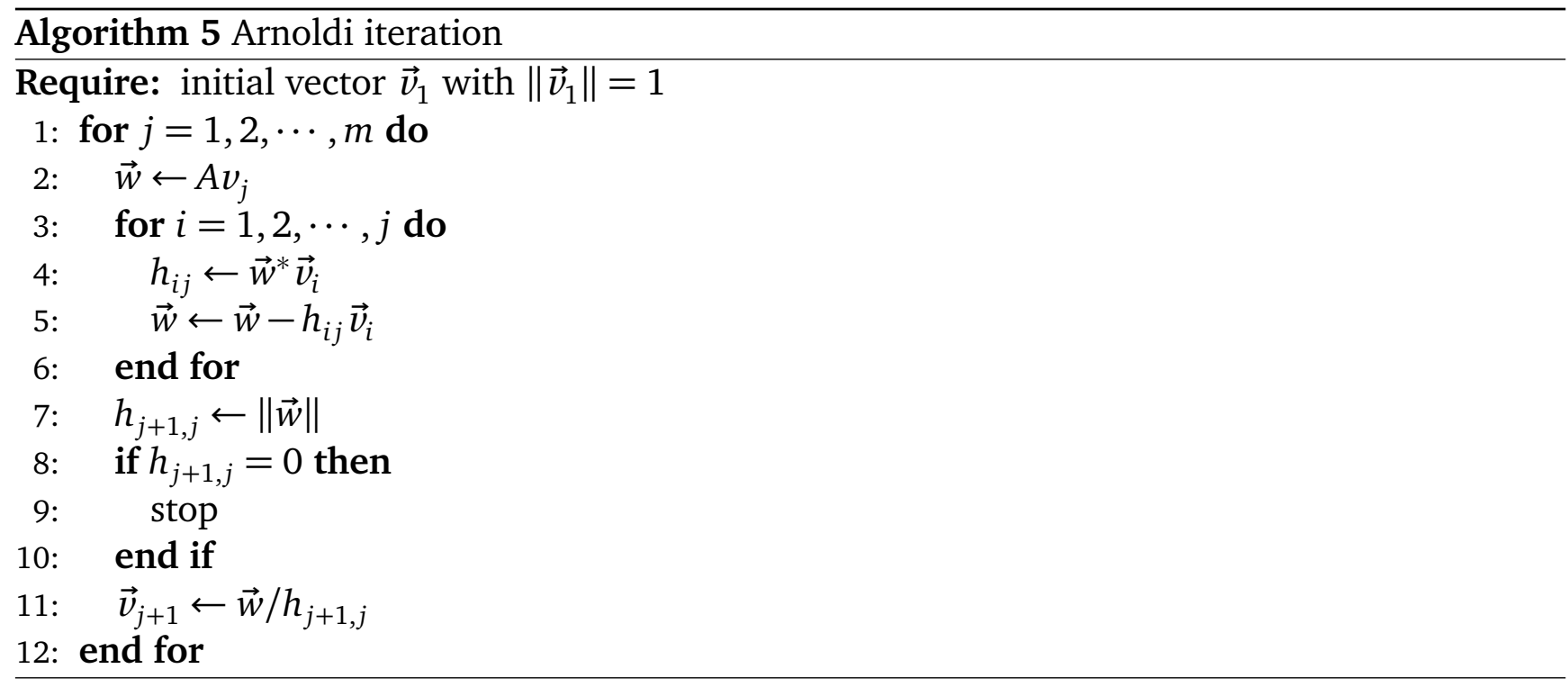


The generated vectors $\vec{v}_{i}(i=1,2, \cdots, m)$ build up an orthonormal basis of the Krylov subspace $\mathscr{K}_{m}\left(A, \vec{v}_{1}\right)$. Moreover, the projection of $A$ onto $\mathscr{K}_{m}\left(A, \vec{v}_{1}\right)$ with respect to $V_{m}=$ $\left\{\vec{v}_{1}, \vec{v}_{2}, \cdots, \vec{v}_{m}\right\}$ is represented by the matrix $H_{m}=V_{m}^{*} A V_{m}$, which is defined by the elements $h_{i, j}$. This matrix $H_{m}$ has upper Hessenberg form [72]. In the explicitly restarted Arnoldi method, after having performed the $m$ steps of algorithm 5 , the approximate eigenpair $(\vec{y}, \theta)$ for the rightmost eigenvalue is computed according to the Rayleigh Ritz approach. If its accuracy is not yet satisfactory, the Arnoldi iteration is repeated with the initial vector $\vec{v}_{1}=\vec{y}$.

In contrast, the Lanczos method generates a pair of biorthogonal bases of the Krylov subspaces $\mathscr{K}_{m}\left(A, \vec{v}_{1}\right)$ and $\mathscr{K}_{m}\left(A^{*}, \vec{w}_{1}\right)$. The biorthogonal projection of $A$ is then represented by a tridiagonal matrix $T_{m}=W_{m}^{*} A V_{m}$, with $W_{m}=\left\{\vec{w}_{1}, \vec{w}_{2}, \cdots, \vec{w}_{m}\right\}$. Note that for Hermitian eigenvalue problems, the two bases coincide for the choice $\vec{v}_{1}=\vec{w}_{1}$, whereas the Arnoldi matrix $H$ also reduces to tridiagonal form. Consequently, in that case the Lanczos and the Arnoldi methods are identical. For a detailed comparison of the two methods is referred to [86]. Finally, it should be noted that there is a variety of more sophisticated variants of both algorithms including deflation and implicit restart techniques available, which, for instance, are discussed in [72, 74].

\subsubsection{The Jacobi-Davidson method}

The Jacobi-Davidson (JD) method [87] is a powerful iterative approach for the computation of one or several eigenvalues and associated eigenvectors of a large sparse system. Though there are different variants of the JD-algorithm, which are adapted to the type of the eigenvalue problem under consideration, they all share the same principle regarding the expansion of the subspace by means of the JD-correction equation (3.158), which was introduced in subsection 3.5.5.2. In this subsection we limit ourselves to the discussion of the algorithm variants that have actually been implemented and tested as part of this thesis. Among those are JD-methods for the Hermitian eigenvalue problem employing either the Rayleigh-Ritz or the harmonic Ritz method as well as a version for non-Hermitian matrices involving partial Schur-forms combined with the Rayleigh-Ritz method. The following schemes are basically adopted from [74], where also complete templates suited for a direct implementation can be found.

\section{The Jacobi-Davidson method for Hermitian eigenvalue problems}

The JD algorithm for Hermitian eigenvalue problems using the Rayleigh-Ritz method for the extraction of eigenpairs including restart and deflation takes the form described in algorithm 6.

A simplified flowchart of the algorithm is also shown in figure 3.16. On convergence, the eigenvectors are available as the columns of $\widetilde{X}$, the eigenvectors as the $\widetilde{\lambda}_{k}$. Due to the convergence properties of the Ritz values (cf. subsection 3.5.5.1), this scheme is favorable for the computation of exterior eigenvalues. In contrast, the variant with harmonic Ritz values aims at the efficient computation of interior eigenvalues. The algorithm for the computation of the $k_{\max }$ eigenvalues at the right side nearest to the target value $\tau$ is described in algorithm 7 .

As both variants with the Rayleigh-Ritz method and the harmonic Ritz values for the extraction from the subspace have been implemented, comparisons were made in real applications for the computation of the lowest nonzero eigenmodes. Hence, the desired eigenvalues are located inside the spectrum albeit usually well separated from the static modes. To sum up, the following conclusions are drawn: In general, both approaches work well for the computation of the lowest eigenmodes exhibiting similar fast convergence. Under certain circumstances, however, 


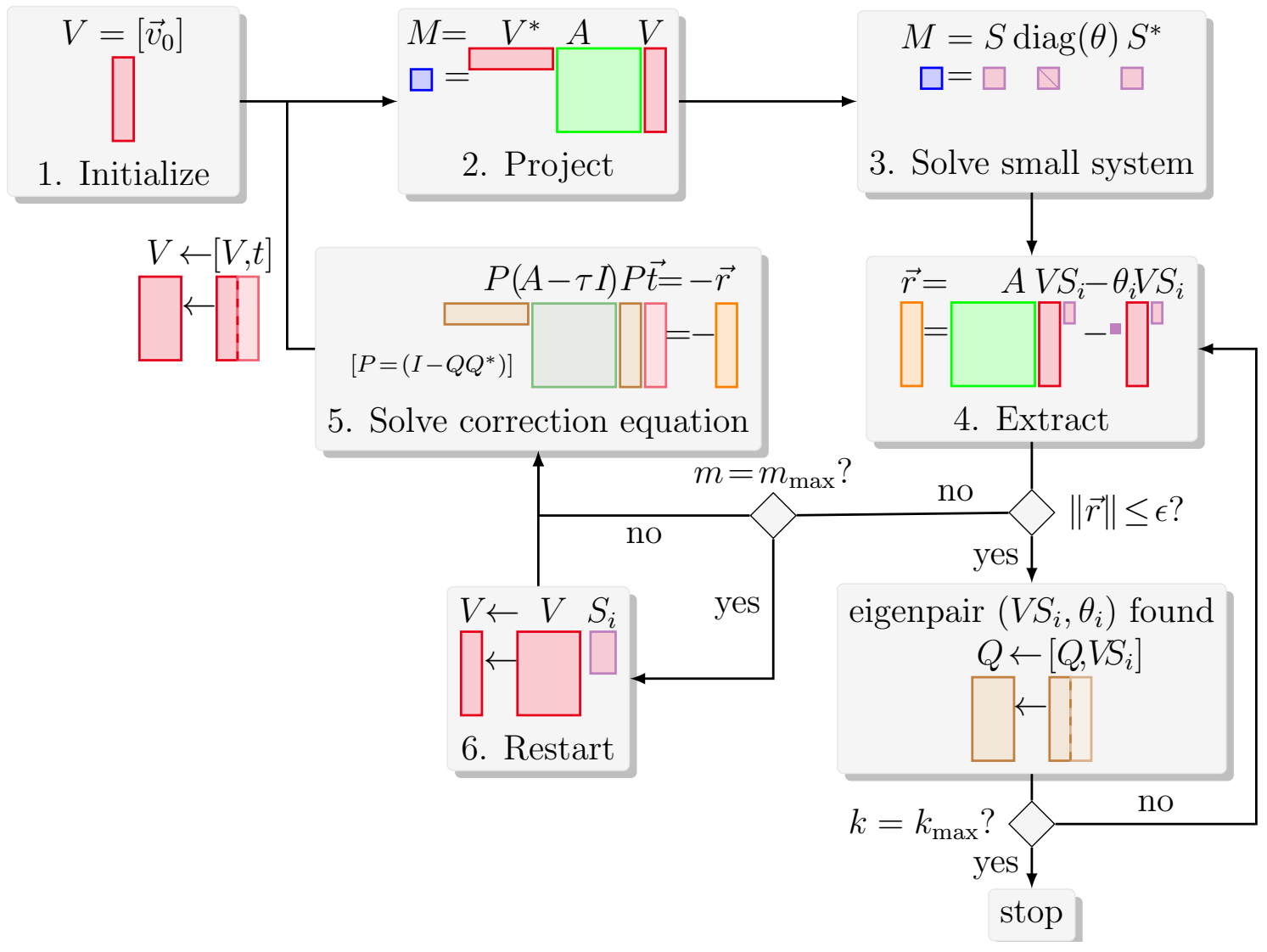

Figure 3.16.: Simplified flowchart of the JD-algorithm for Hermitian eigenvalue problems using the Rayleigh-Ritz method for the extraction of eigenpairs including restart and deflation. After initialization of the projection matrix $V$ (step 1), the main loop of the algorithm consists of projection of the system matrix $A$ to a small system $M$ (step 2), eigendecomposition of $M$ (step 3), Rayleigh-Ritz extraction with subsequent calculation of the residual (step 4) and the extension of $V$ with the solution vector $\vec{t}$ of the correction equation (step 5). If the maximal dimension $m_{\max }$ of the small subspace is reached, a restart is performed (step 6). 
Algorithm 6 Jacobi-Davidson method for the computation of $k_{\max }$ exterior eigenvalues for a Hermitian eigenvalue problem

Require: initial guess for eigenvector $\vec{\nu}_{0}$, target value $\tau$, number of desired eigenvalues $k_{\max } \geq 1$

1: $\vec{t} \leftarrow \vec{v}_{0}, k \leftarrow 0, m \leftarrow 0$

2: loop

3: $\quad$ orthonormalize $\vec{t}$ with respect to current search subspace $V=\left[\vec{v}_{1}, \cdots, \vec{v}_{m}\right]$

4: $\quad m \leftarrow m+1$

5: $\quad M \leftarrow V^{*} A V$

6: $\quad$ compute eigendecomposition $M=S \Theta S^{*}$ with the sorted eigenvalues: $\left|\theta_{1}-\tau\right| \leq\left|\theta_{2}-\tau\right| \leq \ldots$

7: $\quad$ apply Rayleigh-Ritz procedure and compute residuum: $\vec{r} \leftarrow A V \vec{s}_{1}-\theta_{1} V \vec{s}_{1}$

8: $\quad$ while $\|\vec{r}\| \leq \epsilon$ do

9: $\quad$ lock eigenpair: $\tilde{\lambda}_{k+1} \leftarrow \theta_{1}, \widetilde{X} \leftarrow\left[\tilde{X}, V \vec{s}_{1}\right], k \leftarrow k+1$

10: $\quad$ if $k=k_{\max }$ then

11: $\quad$ stop

12: $\quad$ end if

13: $\quad$ reduce search subspace: $V \leftarrow V\left[\vec{e}_{2}, \cdots, \vec{e}_{m}\right], S \leftarrow\left[\vec{s}_{2}, \cdots, \vec{s}_{m}\right], m \leftarrow m-1$

14: apply Rayleigh-Ritz procedure and compute residuum for next eigenpair: $\vec{r} \leftarrow A V \vec{s}_{1}-\theta_{1} V \vec{s}_{1}$

15: end while

16: $\quad$ if $m \geq m_{\max }$ then

17: $\quad$ restart: $V \leftarrow V\left[\vec{s}_{1}, \cdots, \vec{s}_{\text {min }}\right], m \leftarrow m_{\text {min }}$

18: end if

19: $\quad \theta \leftarrow \theta_{1}, Q \leftarrow[\tilde{X}, \vec{u}]$

20: $\quad$ solve $\vec{t} \perp Q$ (approximately) from: $\left(\mathbb{1}-Q Q^{*}\right)(A-\theta \mathbb{1})\left(\mathbb{1}-Q Q^{*}\right) \vec{t}=-\vec{r}$

21: end loop

stability issues are observed for the method involving the harmonic Ritz values. It may happen, possibly due to round-off errors, that the relation $W=(A-\tau \mathbb{1}) V$ is not satisfied with sufficient accuracy anymore, which may lead to stagnation of the JD-algorithm. What is more, in such a case the residuum is not calculated correctly in step (8) of algorithm 7. This may finally lead to the accepting of false eigenvectors, often with an eigenvalue near the target value. Those eigenvectors are, however, exposed as false later on in a verification test by using directly $(A-\tau \mathbb{1}) \mathrm{V}$ instead of $W$ for the computation of the residuum. Such stability problems are more severe when the target value is chosen close to the actual eigenvalue, which in turn normally means that the estimate for the eigenvalue is much more accurate than the estimate for the eigenvector. Similar issues are also know from literature [88]. The easiest way to circumvent those problems is to choose the target value further away from the eigenvalue to obtain convergence at all. This implies, however, that the speed of convergence decreases, too. In the end, the JD-algorithm employing the Rayleigh-Ritz method is the preferred choice for the application throughout this thesis.

\section{The Jacobi-Davidson method for non-Hermitian eigenvalue problems}

For the application of the JD-method for non-Hermitian eigenvalue problems obviously the non-Hermitian structure has to be taken into account for the construction of the reduced ma- 


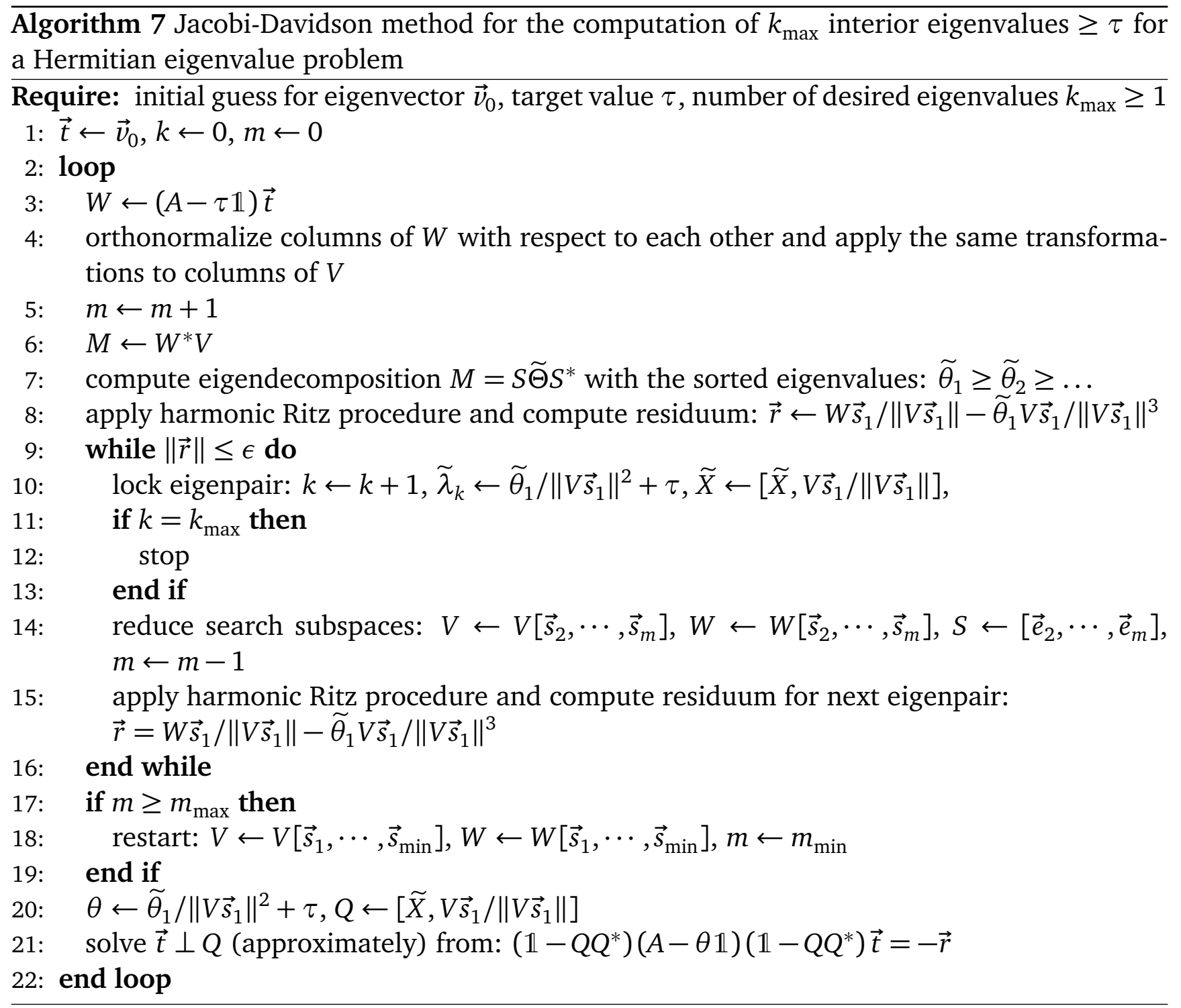

trix as well as for the computation of its eigenvectors. Moreover, restarts and deflation can be included by using an orthonormal set of Schur-vectors (cf. subsection 3.5.3.2). That is to say, instead of calculating the eigenvectors of the large system immediately, its partial Schurdecomposition is computed at first. On completion, the actual eigenvectors are obtained by reordering as explained in section 3.5.4. For this reason, this variant of the method is also referred to as JD-style QR algorithm [89]. As for the variant for Hermitian problems, it can be combined with the Rayleigh-Ritz or harmonic Ritz method for the extraction of the eigensolutions from the subspace. For the reasons discussed in the end of the last paragraph, here only the one using the Rayleigh-Ritz procedure is presented and later on implemented. It takes the form specified in algorithm 8.

\section{Convergence of the Jacobi-Davidson method}

The convergence of the JD-approach is governed by the expansion of the subspace by means of the JD correction equation. As is shown in [90], the effective expansion vector is $(A-\mathbb{1} \theta)^{-1} \vec{u}$. Hence, if the shift $\theta$ is chosen as the target value $\tau$, this vector is identical to the one of the inverse iteration (cf. subsection 3.5.2.2). Similarly, if $\theta$ is set to the Rayleigh quotient, the ex- 
Algorithm 8 Jacobi-Davidson method for the computation of $k_{\max }$ exterior eigenvalues for a non-Hermitian eigenvalue problem

Require: initial guess for eigenvector $\vec{v}_{0}$, target value $\tau$, number of desired eigenvalues $k_{\max } \geq 1$

1: $\vec{t} \leftarrow \vec{v}_{0}, k \leftarrow 0, m \leftarrow 0, Q \leftarrow[], R \leftarrow[]$

2: loop

3: $\quad$ orthonormalize $\vec{t}$ with respect to current search subspace $V=\left[\vec{v}_{1}, \cdots, \vec{v}_{m}\right]$

4: $\quad m \leftarrow m+1$

5: $\quad M \leftarrow V^{*} A V$

6: $\quad$ compute Schur decomposition $M=S T S^{*}$ with ordering: $\left|T_{i, i}-\tau\right| \leq\left|T_{i+1, i+1}-\tau\right| \leq \ldots$

7: $\quad$ apply Rayleigh-Ritz procedure and compute residuum: $\vec{r} \leftarrow\left(1-Q Q^{*}\right)\left(A V \vec{s}_{1}-T_{1,1} V \vec{s}_{1}\right)$

8: $\quad$ while $\|\vec{r}\| \leq \epsilon$ do

9: $\quad$ lock Schur vector: $\widetilde{a} \leftarrow Q^{*}\left(A V \vec{s}_{1}-T_{1,1} V \vec{s}_{1}\right), R \leftarrow\left[\begin{array}{cc}R & \widetilde{a} \\ 0 & T_{1,1}\end{array}\right], Q \leftarrow\left[Q, V \vec{s}_{1}\right], k \leftarrow k+1$

10: $\quad$ if $k=k_{\max }$ then

11: $\quad$ stop

12: $\quad$ end if

13: $\quad$ reduce search subspace: $V \leftarrow V\left[\vec{s}_{2}, \cdots, \vec{s}_{m}\right], S \leftarrow\left[\vec{e}_{2}, \cdots, \vec{e}_{m}\right]$,

$T \leftarrow$ lower $m \times m$ block of $T, m \leftarrow m-1$

14: $\quad$ apply Rayleigh-Ritz procedure and compute residuum: $\vec{r} \leftarrow\left(1-Q Q^{*}\right)\left(A V \vec{s}_{1}-T_{1,1} V \vec{s}_{1}\right)$

15: end while

16: $\quad$ if $m \geq m_{\max }$ then

17: $\quad$ restart: $V \leftarrow V\left[\vec{s}_{1}, \cdots, \vec{s}_{\min }\right], m \leftarrow m_{\min }$

18: end if

19: $\quad \theta \leftarrow T_{1,1}, \widetilde{Q} \leftarrow[\tilde{X}, \vec{u}]$

20: $\quad$ solve $\vec{t} \perp \widetilde{Q}$ (approximately) from: $\left(\mathbb{1}-\widetilde{Q} \widetilde{Q}^{*}\right)(A-\theta \mathbb{1})\left(\mathbb{1}-\widetilde{Q} \widetilde{Q}^{*}\right) \vec{t}=-\vec{r}$

21: end loop

pansion vector is the same as for the Rayleigh quotient iteration (RQI) (cf. subsection 3.5.2.3). Hence, depending on the choice of $\theta$, the JD procedure may be interpreted as a subspace accelerated inverse iteration or RQI [90]. This observation allows to draw the following conclusions: Firstly, it is sensible to perform the initial JD-iterations with $\theta=\tau$ in order to enforce the convergence towards the eigenvalue closest to $\tau$. After some iterations the shift should be updated with the current Rayleigh quotient to benefit from the fast convergence of the Rayleigh quotient iteration in the vicinity of the exact solution. Secondly, if the correction equation is solved exactly, the JD methods mimics the convergence of the inverse iteration or RQI, again depending on the selection of the shift. Hence, in the latter case it features an asymptotically quadratic and for Hermitian matrices even cubic convergence of the Ritz-values towards exterior eigenvalues of $A$ [74]. The same applies for convergence of Harmonic Ritz values towards eigenvalues $\lambda$ closest to the shift (as long as $\lambda \neq \tau$ ).

Taking this into account, in this implementation the shift is chosen as $\theta=\tau$ for the computation of the first eigenvector as long as the relative deviation between the current estimate for the eigenvalue and $\tau$ is greater than 0.05; for all subsequent eigenvalues the current best estimate is always chosen for $\theta$. Depending on the spectrum of the eigenvalues, it might be beneficial to work with different selection criteria. 


\section{Preconditioning}

In practice, the correction equation is solved only approximately. Since both the rate of the convergence of the eigenvalues strongly depends on the accuracy of its solution and the solution of the correction equation is by far the computationally most expensive part of the whole JDalgorithm, it is worthwhile to spend some effort on the choice of an appropriate preconditioner. Among the most common preconditioners suited for this purpose are the Jacobi preconditioner as well as the incomplete or complete LU decomposition [51]. For the Jacobi type just the main diagonal of the system matrix is taken as the preconditioner. Hence, this type can be constructed with very low computational effort and has very moderate memory requirements. The opposite is true for the LU decomposition. Here, the time spent for the decomposition amounts a significant part to the total computation time. Moreover, due to the usually large fill-in, the memory requirement may become prohibitively large. Thus, the incomplete LU decomposition with an adjustable fill-in might be a good compromise. Yet, as confirmed by own studies, it is not recommended in practice. The reason is that, on the one hand, the observed convergence is not significantly better than for simple Jacobi preconditioning for a low fill-in. On the other hand, due to the mature state of libraries available for the complete LU decomposition, already for a moderate fill-in the incomplete variant is not superior anymore to the complete one in terms of performance (cf. also [91]). As a rule of thumb, as long as sufficient main memory is available, it is thus recommendable to compute the LU decomposition of the system matrix at the first time the correction equation is to be solved. Yet, often satisfactory convergence is still observed for all subsequent steps when the same preconditioner is kept if only a few eigenvalues are requested. The LU factorization is only updated when convergence problems are encountered. In fact, the update is computed significantly faster than the first decomposition since the information for the symbolic factorization can be reused and no additional memory needs to be allocated. Nevertheless, alternatively also Jacobi preconditioning leads to convergence of the JD algorithm even for non-diagonal gyrotropic material matrices. It may thus be the method of choice for very large problems, which could not be solved anymore with LU preconditioning due to memory restrictions.

Besides, the preconditioner for gyroelectric materials with the permittivity tensor of definition (3.30) can be obtained in a more efficient way by employing the relation (as published in [59])

$$
\left(\mathbf{M}_{\overleftrightarrow{\varepsilon}}^{-1} \widetilde{\mathbf{C}} \mathbf{M}_{\stackrel{\leftrightarrow}{\leftrightarrow}} \mathbf{C}-\lambda \mathbb{1}\right)^{-1}=\left(\widetilde{\mathbf{C}} \mathbf{M}_{\stackrel{\leftrightarrow}{\leftrightarrow}} \mathbf{C}-\mathbf{M}_{\overleftrightarrow{\varepsilon}} \cdot \lambda \mathbb{1}\right)^{-1} \mathbf{M}_{\stackrel{\varepsilon}{\leftrightarrow}}
$$

This is advantageous as one can avoid the computation of the inverse of the expression on the left hand side, which itself includes the inverse of the permittivity matrix. Since $\mathbf{M}_{\overleftrightarrow{\varepsilon}}^{-1}$ has by far more nonzero elements than the directly constructed non-inverse permittivity matrix, the computation of the right hand side of equation (3.160) is much more efficient than the construction of the inverse according to the expression on the left hand side.

\section{Stopping criterion}

It is sufficient to solve the JD-correction equation with a low accuracy for the first few JDiterations but a more accurate solution is required with an improved estimate for the eigenpairs. Hence, a dynamically adaption of the stopping criterion for the JD-correction equation is favorable. Similar to [92], the stopping criterion

$$
\left\|\widetilde{r}_{j}\right\|<\gamma^{n}\left\|\widetilde{r}_{0}\right\| \quad \vee \quad n \geq \max \left(n_{\min }, n_{\text {inc }} \cdot n\right)
$$


is used, where $\left\|\widetilde{r}_{j}\right\|$ is the residual after the $j$-th step of the inner method for the solution of the correction equation, $n$ the current number of JD-iterations, and $0<\gamma<1, n_{\min }$ and $n_{\text {inc }}$ parameters to be set adequately. In this implementation, these values are chosen as $\gamma=0.1$, $n_{\min }=10$ and $n_{\text {inc }}=1$ if LU preconditioning is used and $\gamma=0.5, n_{\min }=100$ and $n_{\text {inc }}=5$ for Jacobi preconditioning.

\subsubsection{Implementation of the eigenvalue solver}

Of the different approaches for the computation of eigensystems introduced in the last subsection, the JD-type schemes appear to be best suited for the application in this thesis because we are interested in the computation of only a few complex eigenpairs of large sparse system matrices. Moreover, the JD-algorithm allows an implementation for an efficient parallel computation on a cluster with distributed memory machines. In concrete terms, all the three mentioned JD-variants, algorithms 6, 7 and 8, have been successfully implemented. Some details on the implementation, in particular including remarks on efficiency, are discussed in subsection 3.5.6.1. Furthermore, subsection 3.5.6.2 deals with the application to nonlinear eigenvalue problems. For verification the numerically computed eigenmodes are compared to reference solutions for a linear eigenproblem as well as for nonlinear ones including also gyrotropic materials in subsection 3.5.6.3.

\subsubsection{Implementation details}

\section{General}

The implementation in the programming language $\mathrm{C} / \mathrm{C}++$ is based on the PETSc suite $[46,47]$. Hence, built-in PETSc routines are employed for the solution of the JD-correction equation. Yet, the LU factorization as preconditioner is computed with the external package SuperLU_DIST [93], which is optimized for distributed memory machines. For alternative Jacobi preconditioning, an own implementation, which does not rely on PETSc's KSP-wrapper, is used. This leads to an approximately 5\% faster application of the preconditioner compared to PETSc's built-in Jacobi preconditioner due to reduced overhead. The eigen- or the Schur decompositions of the small projected problem are efficiently performed with adequate LAPACK-subroutines [79]. This requires that the data are provided in continuous chunks of memory, which is further discussed in the next paragraph.

\section{Memory management}

An appropriate memory management is the key for a high performance computation and thus deserves special attention. In the JD-algorithm, the size of the matrices for the subspaces and the small projected system and their associated vectors changes from iteration to iteration. It should, however, be refrained from allocating and freeing the required memory again and again. Instead, the maximal required memory is only allocated once in the very beginning. The large projection matrices are not created as PETSc-matrices but rather as arrays of (PETSc-) vectors, which allows to adjust the number of matrix columns very efficiently. What is more, the memory of each array of vectors is allocated as one continuous chunk of memory such that individual vector elements can also be accessed directly as for standard C-style two-dimensional arrays. This also enables to benefit from the direct use of BLAS Level 3 routines $[94,95]$ for example at 
the update of the subspace matrices at restart or reduction of the search subspace. Surprisingly, profiling revealed that the update function that makes use of the BLAS Level 3 routines is even slower than an own implementation of the update function with the same functionality by hand. It is speculated that this is by virtue of very good compiler optimizations, which might be more effective for the latter. Moreover, as already mentioned in the previous paragraph, the allocation of the memory for the small projected system as one continuous chunk is a requirement for the usage of LAPACK-subroutines.

\section{Matrix-free methods}

PETSc supports matrix-free methods, which do not require explicit storage of the matrices [47]. The implementation of the eigensolver makes wide use of this functionality. Firstly, the system matrix, whose eigenvalues are to be determined, is not stored as such but is regarded as the product of its individual submatrices (cf. Eq. (3.114)). Hence, every time a matrix-vector product is performed, it is calculated as the sequence of matrix-vector products, i.e. as

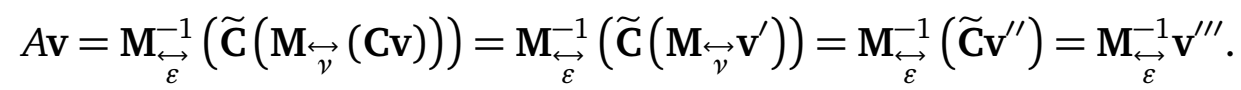

This is indeed more efficient than the multiplication with the explicitly assembled system matrix instead since the system matrix has much more nonzero elements than the sum of the nonzeros of the individual submatrices. The advantage of the matrix-free representation is even more evident for nonlinear eigenvalue problems where the system matrix would have to be re-assembled in each nonlinear iteration (cf. the following subsection 3.5.6.2). Secondly, all topological matrices (cf. subsection 3.4.5) are not stored as standard PETSc-matrices. Since the topological matrices are built up only of the elements \pm 1 but PETSc only supports the double (or complex) data type for matrices, this would be an enormous waste of memory. In the implementation used in this thesis, the following hybrid approach is pursued: Each topological matrix is stored as a (possibly MPI-type) sparse matrix. Yet, the information on the sign of the non-vanishing element is encoded in the sign of the column index of the sparse storage format (cf. section 3.3). Additionally, the function for the matrix-vector product is implemented such that relevant vector elements are just added or subtracted instead of additionally being multiplied with \pm 1 . Of course, one might also think of a completely matrix-free format for the topological matrices without explicit storage at all. One would then have to determine the indices of the \pm 1 elements at the time of matrix-vector multiplication. For the special case of the first ordering of the DOFs as in the standard FIT (cf. subsection 3.4.5.1) with no parallel computing, the indices are known immediately thanks to the clear band structure. However, in any other case, i.e. for a computation with more than one process, for ordering 2 (cf. subsection 3.4.5.2), if the zero structure of the topological matrices (cf. subsection 3.4.7.1) is taken into account, or for the reduced grid (cf. section 3.4.7), this involves some kind of computations. Clearly, the calculation of the indices "on the fly" would be significantly slower than the explicit storage of the elements. To conclude, the before-mentioned implementation is regarded as the best compromise between computational speed, memory requirements and ease of implementation.

\section{Efficiency considerations for gyromagnetic and gyroelectric materials}

The following considerations regarding peculiarities of an efficient eigenmode computation with both gyromagnetic and gyroelectric materials or only one of the two substances are also published in [59]. Since the inverse permittivity matrix enters in the system matrix of the curlcurl system (3.114) but only its non-inverse expression is available directly, a LU decomposition 
of $\mathbf{M}_{\stackrel{\varepsilon}{\leftrightarrow}}$ is performed. This leads to the unfortunate but for robustness sake still acceptable much larger computational effort as for models with only gyromagnetic materials. Using directly a material matrix for the inverse permittivity instead might seem to be a computationally cheap alternative. However, it is found that a simple construction of the inverse permittivity matrix analogous to definition (3.98) but with the components of the permittivity tensor replaced with its corresponding components of the inverse tensor, which is one of the schemes presented in [96] for the finite-difference time-domain method, does not lead to the correct eigenvalues in the limit of fine discretizations. Given that the continuity conditions of the electric field cannot immediately be fulfilled in the FIT using that definition, the discrepancy is, however, not surprising. An alternative approach could also be the formulation as a generalized eigenvalue problem, which can be obtained by multiplying equation (3.114) with $\mathbf{M}_{\stackrel{\varepsilon}{\leftrightarrow}}$ from the left. However, the solution of the generalized eigenproblem would require a modified algorithm. Yet, since cavities in real applications are filled with materials which exhibit only either gyromagnetic or gyroelectric characteristics, the problem can be circumvented also for purely gyroelectric substances. To this end, the allocation of the electric and magnetic DOFs in the FIT (cf. subsection 3.4.2) can be swapped. Then it is possible to construct a material matrix directly for the inverse permittivity in an analogous manner as for the inverse permeability for the standard allocation.

\subsubsection{Approach for nonlinear eigenvalue problems}

The JD-algorithms presented in subsection 3.5.5.4 are suited for the solution of standard linear eigenvalue problems. However, due to the frequency dependence of the material matrices of gyrotropic materials (cf. subsection 3.4.8), nonlinear eigenproblems arise for, e.g., ferriteloaded cavity resonators. Though JD-type algorithms modified for nonlinear problems have been developed [97], a simple iterative substitution scheme is used for this purpose throughout this thesis. In fact, this approach proves to be efficient in practice by reusing as much information as possible from preceding steps. Thus, previously computed eigenvectors are set as initial vectors for the JD-algorithm in subsequent nonlinear iterations. Consequently, a much smaller number of JD-iterations is required from the second nonlinear iteration on. Moreover, the preconditioner is kept for all nonlinear iterations. What is more, the iterative substitution approach requires to select the currently desired eigenmode out of all computed ones after each solution of a linear problem. To this end, it is worthwhile not only to compare the eigenvalues but also to calculate the inner products of current eigenvectors with already converged ones. In case of non-Hermitian eigenproblems, the (orthogonal) vectors of the computed partial Schur form are used instead to calculate the inner products. This way the solver is also capable of distinguishing different modes of degenerated eigenvalues both for Hermitian and non-Hermitian problems. Consequently, it is ensured that all eigenvectors associated with the same eigenvalue can be found even for nonlinear eigenvalue problems. 


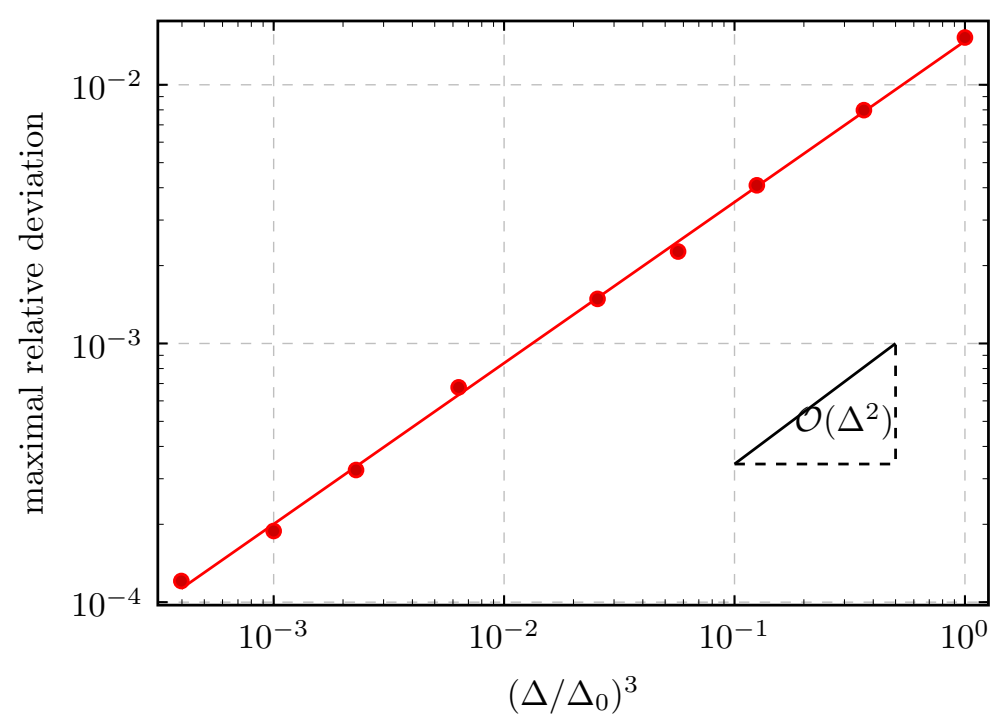

Figure 3.17.: Maximal relative frequency deviation between the numerical computation and the analytical value $f_{2,1}$ for any of the five degenerated modes of the second lowest resonance frequency as a function of the normalized step size $\Delta / \Delta_{0}$ for the spherical resonator.

\subsubsection{Verification}

\section{Verification of the linear eigensolver}

For the verification of the linear eigenvalue solver, a spherical cavity resonator with radius $r=1 \mathrm{~m}$ is considered. The eigenfrequencies $f_{n, m}$ are determined by the roots of the equation [98]

$$
\sqrt{x_{n, m}} J_{m+\frac{1}{2}}\left(x_{n, m}\right)=0,
$$

where $J_{n}$ is the Bessel function of the first kind of order $n$ and $x_{n, m}=k_{n, m} r=2 \pi f_{n, m} r / c_{0}$ the $m$-th zero of $J_{n}$ with the speed of light in vacuum $c_{0}$ and the (angular) wavenumber $k_{n, m}$. The lowest resonance frequency at $f_{1,1}=130.912 \mathrm{MHz}$ has a degree of degeneracy of three, whereas the second lowest one is found at $f_{2,1}=184.662 \mathrm{MHz}$ with a degree of degeneracy of five. Since no nonlinear or tensorial material is involved, the material matrices are directly imported to the own implementation of the eigenvalue solver from CST STUDIO SUITE® [48] with activated support of the Perfect Boundary Approximation ${ }^{\circledR}$ (PBA). Figure 3.17 shows the maximal relative frequency deviation between the numerical computation and the analytical value for any of the five degenerated modes of the second lowest resonance frequency as a function of the normalized step size. As expected for PBA-meshing, convergence towards the reference values with almost second order is observed.

\section{Verification of the nonlinear eigensolver with gyrotropic materials}

The nonlinear eigensolver is verified through the computation of eigenmodes of biased cavity resonators of cylindrical and rectangular shape, which are filled with gyrotropic material.

Cylinder resonator: Firstly, a cylindrical resonator (radius $r=1 \mathrm{~m}$, length $l=2 \mathrm{~m}$ ) longitudinally biased by a homogeneous static magnetic field is considered. The eigensolutions can be calculated semi-analytically as briefly outlined in the following. The basic theory is due to Kales 
[99], who formulated an analytical characteristic equation for cylindrical waveguides filled with media which is gyromagnetic only. This procedure was then modified for the case of cylinder resonators by Chinn et al. [100]. Moreover, Trier generalized Kales' theory to waveguides containing material with both gyromagnetic and gyroelectric properties [101]. His results are the basis for the calculation of the eigenmodes for the setup described above for a permeability tensor of the form (2.22) and a permittivity tensor as given in equation (2.42). Following the notation of [100], modes which become TE (TM) modes in the limit of vanishing $\kappa$ and $\varepsilon_{2}$ are denoted as $\mathrm{HE}(\mathrm{EH})$ modes. The eigenfrequencies of $\mathrm{HE}$ and $\mathrm{EH}$ modes are the roots of the characteristic equation given in [101, p. 337], which can be calculated by standard numerical algorithms. Furthermore, the ones of the TM modes are implicitly determined by

$$
\omega_{n, m, 0}=\frac{x_{n, m}}{r} \times\left(\varepsilon_{3}\left(\omega_{n, m, 0}\right) \frac{\left(\mu_{\text {diag }}\left(\omega_{n, m, 0}\right)\right)^{2}-\left(\kappa\left(\omega_{n, m, 0}\right)\right)^{2}}{\left(\mu_{\text {diag }}\left(\omega_{n, m, 0}\right)\right)^{2}}\right)^{-1 / 2},
$$

where $x_{n, m}$ represents the $m$-th zero of the Bessel function of order $n$.

To begin with, the cylinder is supposed to be filled with a lossless, purely gyromagnetic material whose magnetic properties can be described by the Polder tensor (cf. Eq. (2.22)) and whose relative permittivity is $\varepsilon_{r}=1$. The bias magnetic field strength is chosen as $H_{0}=2750 \mathrm{~A} / \mathrm{m}$, the saturation magnetization as $M_{\mathrm{sat}}=20 \times 10^{3} \mathrm{~A} / \mathrm{m}$. Furthermore, the Landé $g$-factor occurring in the gyromagnetic ratio is set to $g=2.1$. With these parameters, the 10 lowest eigenmodes, which are computed numerically with the new implementation of the nonlinear eigensolver described before, are compared to the semi-analytically calculated values, which are listed in table 3.4. Furthermore, to ensure a reliable verification of the eigenmodes, the field distributions have been investigated additionally. Figure 3.18(a) shows the convergence of the numerically computed eigenvalues towards the semi-analytical ones with order 1 as is expected for the approximation of rounded geometries with a staircase filling.

To further test the construction of the permeability tensor for an arbitrarily oriented magnetic bias field, the numerical computation is additionally carried out for different orientations of the cylinder axis to the coordinate axes keeping the external magnetic field aligned with the cylinder axis. Note that for the rotated cylinder it is particularly crucial to assign the modes correctly for a reliable verification. If one sorts the eigenmodes simply in ascending order with respect to the eigenvalues, by comparing the field solutions it shows that the order of the modes is different compared to the semi-analytical calculation. This is, however, only the case for discretizations on a rather coarse mesh. By a proper refinement of the grid, the expected order is retained. Taking this into account, good accordance of the numerical values with the analytical results is observed for all tested orientations. This is shown as an example in figure 3.18(b) for the orientation as depicted therein.

In the next verification example, the same setup with the cylinder axis in $z$-direction is picked up again. Yet, it is further assumed that the cylinder material exhibits both gyromagnetic and gyroelectric properties of the form given in subsection 2.2.4. In this example, the (angular) plasma frequency is set to $\omega_{\mathrm{p}}=2 \pi \times 15 \mathrm{MHz}$, whereas the (angular) cyclotron frequency $\omega_{\mathrm{b}}=B_{0} e / m_{e}$ is chosen as $425 \mathrm{MHz}$. Again, no losses are included so far, which means that the collision frequency $v_{c}$ vanishes. The results of this example are also published in [59]. Table 3.5 lists the semi-analytically calculated values for the 10 lowest eigenfrequencies for the above parameters. Figure 3.19 shows the convergence of the numerically computed eigenvalues towards the semi-analytical ones with order 1 as expected for the staircase mesh for a cylinder geometry. 
Table 3.4.: Semi-analytically calculated eigenfrequencies $f_{\text {analy }}=\omega_{\text {analy }} /(2 \pi)$ for the 10 lowest modes of the biased cylinder resonator filled with lossless, gyromagnetic material.

\begin{tabular}{c|c|c||c|c|c}
\hline \hline$\#$ & mode & $f_{\text {analy }} / \mathrm{MHz}$ & $\#$ & mode & $f_{\text {analy }} / \mathrm{MHz}$ \\
\hline 1 & $\mathrm{TM}_{0,1,0}$ & 39.57 & 6 & $\mathrm{HE}_{1,1,1}^{-}$ & 66.67 \\
2 & $\mathrm{EH}_{0,1,1}$ & 47.04 & 7 & $\mathrm{HE}_{1,1,1}^{+}$ & 66.72 \\
3 & $\mathrm{TM}_{1,1,0}^{+}$ & 62.28 & 8 & $\mathrm{HE}_{1,1,2}^{-}$ & 76.47 \\
4 & $\mathrm{TM}_{1,1,0}^{-}$ & 62.28 & 9 & $\mathrm{HE}_{1,1,2}^{+}$ & 77.57 \\
5 & $\mathrm{EH}_{0,1,2}$ & 63.56 & 10 & $\mathrm{EH}_{0,1,3}$ & 81.17 \\
\hline \hline
\end{tabular}

(a)
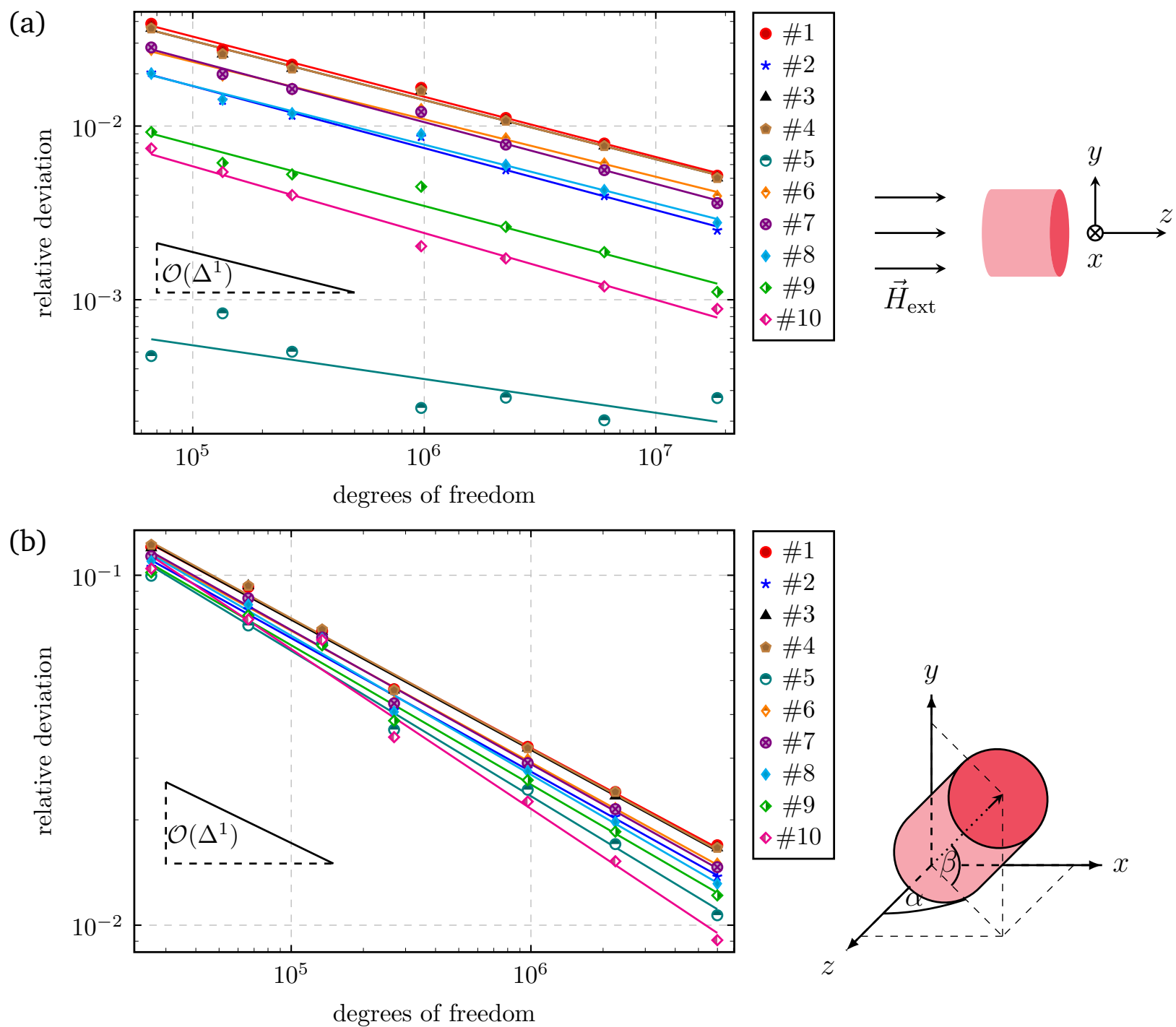

Figure 3.18.: Relative deviation of the numerically obtained value $\omega$ to the analytical result $\omega_{\text {analy }}$ as a function of the DOFs for the 10 lowest eigenfrequencies for a lossless, longitudinally biased, cylindrical resonator filled with gyromagnetic material. The cylinder is aligned with the z-axis (a) or oriented as shown on the right (b), where the cosine of $\beta$, i.e. the angle between the cylinder axis and the $x-z$-plane, is $\sqrt{2 / 3}$ and the angle $\alpha$ between the projection of the cylinder axis onto this plane and the $z$-axis is $45^{\circ}$. 
Table 3.5.: Semi-analytically calculated eigenfrequencies $f_{\text {analy }}=\omega_{\text {analy }} /(2 \pi)$ for the 10 lowest modes of the biased cylinder resonator filled with a material with both gyromagnetic and gyroelectric properties. [59]

\begin{tabular}{c|c|c||c|c|c}
\hline \hline$\#$ & mode & $f_{\text {analy }} / \mathrm{MHz}$ & $\#$ & mode & $f_{\text {analy }} / \mathrm{MHz}$ \\
\hline 1 & $\mathrm{TM}_{0,1,0}$ & 42.27 & 6 & $\mathrm{HE}_{1,1,1}^{-}$ & 68.02 \\
2 & $\mathrm{EH}_{0,1,1}$ & 48.69 & 7 & $\mathrm{HE}_{1,1,1}^{+}$ & 68.07 \\
3 & $\mathrm{TM}_{1,1,0}^{+}$ & 63.99 & 8 & $\mathrm{HE}_{1,1,2}^{-}$ & 77.17 \\
4 & $\mathrm{TM}_{1,1,0}^{-}$ & 63.99 & 9 & $\mathrm{HE}_{1,1,2}^{+}$ & 78.33 \\
5 & $\mathrm{EH}_{0,1,2}$ & 64.19 & 10 & $\mathrm{EH}_{0,1,3}$ & 81.43 \\
\hline \hline
\end{tabular}

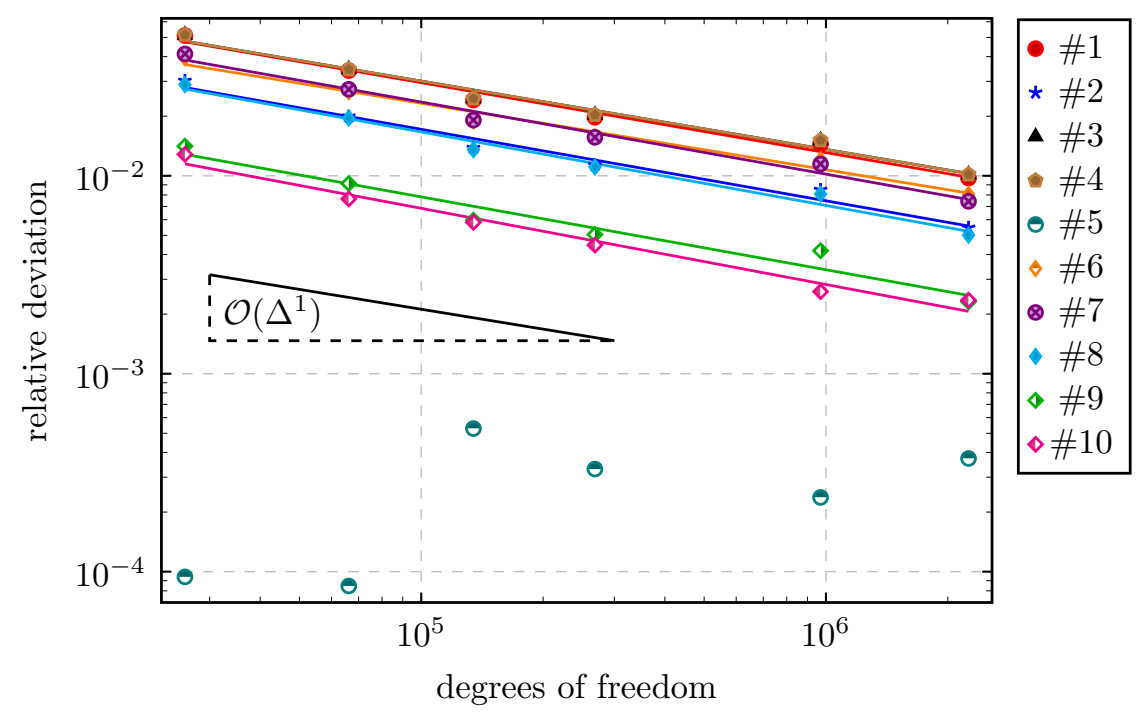

Figure 3.19.: Relative deviation $\left|\omega-\omega_{\text {analy }}\right| / \omega_{\text {analy }}$ of the numerically obtained value $\omega$ to the (semi-)analytical reference value $\omega_{\text {analy }}$ as a function of the DOFs for the 10 lowest modes for a biased, lossless, cylindrical cavity resonator filled with both gyromagnetic and gyroelectric material. [59]

The fifth eigenmode still seems to be in a range below asymptotic convergence and features an unexpected small relative deviation already on a rather coarse mesh.

Rectangular resonator: The second considered resonator is of rectangular shape with dimensions $l_{x}=3.142 \mathrm{~m}, l_{y}=2.718 \mathrm{~m}$ and $l_{z}=0.100 \mathrm{~m}$ in $x$-, $y$ - and $z$-direction, respectively. This verification example is also published in [59]. As before, the homogeneous bias magnetic field oriented in z-direction has a strength of $H_{0}=2750 \mathrm{~A} / \mathrm{m}$. Moreover, the resonator is filled with a material with the same properties as for the last example with the cylinder filled with a material with both gyromagnetic and gyroelectric properties. The only difference is that now also material losses are included by setting $\alpha=0.2$ and $\mathrm{v}_{\mathrm{c}}=10 \mathrm{MHz}$. The geometric dimensions are chosen such that there are only TM modes among the lowest eigenmodes. Their resonance frequencies are identical to the ones of a waveguide with cross-section $l_{x} \times l_{y}$ and can be computed semi-analytically according to the implicit equation

$$
\omega_{n, m, 0}=\frac{k_{x}^{2}+k_{y}^{2}}{\varepsilon_{3}\left(\omega_{n, m, 0}\right)} \times \frac{\left(\mu_{\mathrm{diag}}\left(\omega_{n, m, 0}\right)\right)^{2}}{\left(\mu_{\mathrm{diag}}\left(\omega_{n, m, 0}\right)\right)^{2}-\left(\kappa\left(\omega_{n, m, 0}\right)\right)^{2}}
$$


with the (angular) wavenumbers

$$
k_{x}=\frac{n \pi}{l_{x}}, \quad k_{y}=\frac{m \pi}{l_{y}} \quad\left(n, m \in \mathbb{N}_{0}\right) .
$$

The 10 lowest complex eigenfrequencies as obtained from equation (3.165) are given in table 3.6. As can be seen from figure 3.20, second order convergence of the numerically computed eigenvalues towards the reference values is evident. In fact, both the real part and the imaginary part converge independently with the same convergence order. With the aim of testing also TE modes, the computations are repeated for the same geometry and material but with magnetic boundary conditions instead of electric ones (cf. subsection 3.4.6). For this boundary value problem the eigenfrequencies are implicitly given by

$$
\omega_{n, m, 0}=\frac{k_{x}^{2}+k_{y}^{2}}{\mu_{z}} \times \frac{\left(\varepsilon_{1}\left(\omega_{n, m, 0}\right)\right)^{2}}{\left(\varepsilon_{1}\left(\omega_{n, m, 0}\right)\right)^{2}-\left(\varepsilon_{2}\left(\omega_{n, m, 0}\right)\right)^{2}} .
$$

The 10 lowest modes can also be found in table 3.6. A comparison with numerically computed modes reveals again second order convergence and thus indicates agreement with the semianalytical solution. 
Table 3.6.: Semi-analytically calculated eigenfrequencies $f_{\text {analy }}=\omega_{\text {analy }} /(2 \pi)$ for the 10 lowest modes of the biased rectangular resonator filled with a material with both gyromagnetic and gyroelectric properties. [59]

\begin{tabular}{c|c|c||c|r}
\hline \hline & \multicolumn{2}{|c||}{ electr. boundary cond. } & \multicolumn{2}{c}{ magnetic boundary cond. } \\
$\#$ & mode & $f_{\text {analy }} / \mathrm{MHz}$ & mode & $f_{\text {analy }} / \mathrm{MHz}$ \\
\hline 1 & $\mathrm{TM}_{1,1,0}$ & $29.35+1.03 \mathrm{i}$ & $\mathrm{TE}_{1,1,0}$ & $72.9+403 \times 10^{-6} \mathrm{i}$ \\
2 & $\mathrm{TM}_{1,2,0}$ & $40.87+1.60 \mathrm{i}$ & $\mathrm{TE}_{1,2,0}$ & $110.2+422 \times 10^{-6} \mathrm{i}$ \\
3 & $\mathrm{TM}_{2,1,0}$ & $44.03+1.82 \mathrm{i}$ & $\mathrm{TE}_{2,1,0}$ & $120.1+428 \times 10^{-6} \mathrm{i}$ \\
4 & $\mathrm{TM}_{2,2,0}$ & $52.26+2.52 \mathrm{i}$ & $\mathrm{TE}_{2,2,0}$ & $145.8+448 \times 10^{-6} \mathrm{i}$ \\
5 & $\mathrm{TM}_{1,3,0}$ & $54.69+2.76 \mathrm{i}$ & $\mathrm{TE}_{1,3,0}$ & $153.4+455 \times 10^{-6} \mathrm{i}$ \\
6 & $\mathrm{TM}_{3,1,0}$ & $60.72+3.40 \mathrm{i}$ & $\mathrm{TE}_{3,1,0}$ & $172.1+474 \times 10^{-6} \mathrm{i}$ \\
7 & $\mathrm{TM}_{2,3,0}$ & $63.46+3.72 \mathrm{i}$ & $\mathrm{TE}_{2,3,0}$ & $180.7+484 \times 10^{-6} \mathrm{i}$ \\
8 & $\mathrm{TM}_{3,2,0}$ & $66.74+4.13 \mathrm{i}$ & $\mathrm{TE}_{3,2,0}$ & $190.9+496 \times 10^{-6} \mathrm{i}$ \\
9 & $\mathrm{TM}_{1,4,0}$ & $69.19+4.45 \mathrm{i}$ & $\mathrm{TE}_{1,4,0}$ & $198.6+506 \times 10^{-6} \mathrm{i}$ \\
10 & $\mathrm{TM}_{3,3,0}$ & $75.56+5.36 \mathrm{i}$ & $\mathrm{TE}_{3,3,0}$ & $218.7+536 \times 10^{-6} \mathrm{i}$ \\
\hline \hline
\end{tabular}

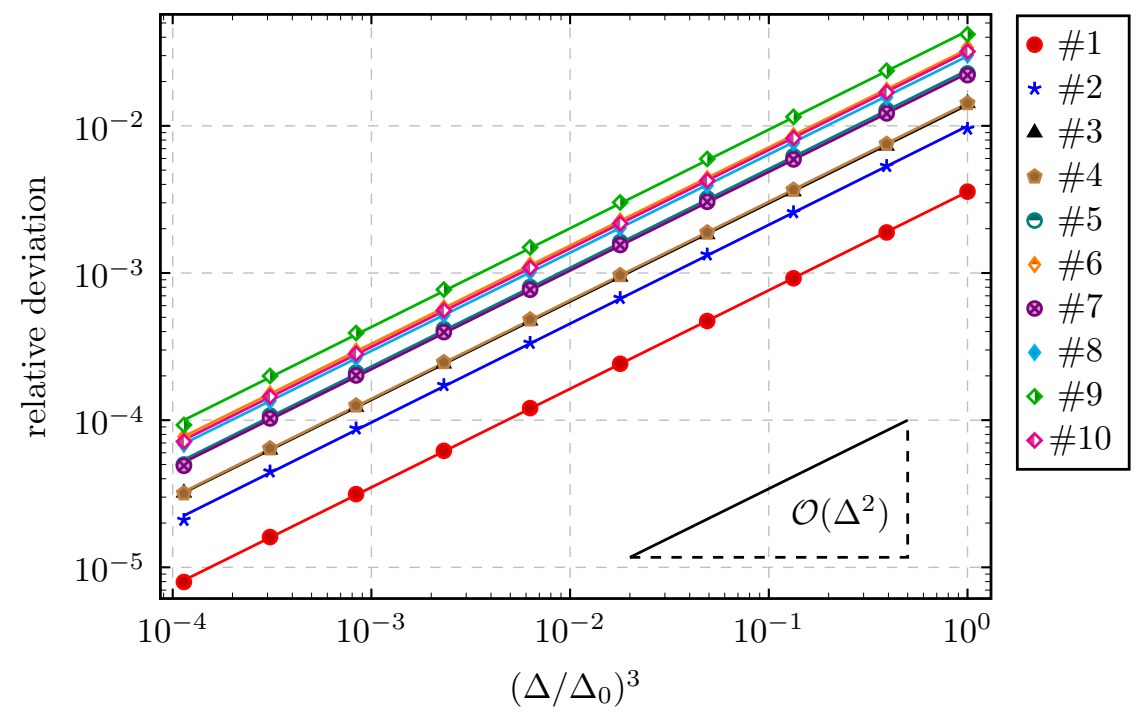

Figure 3.20.: Relative deviation ||$\omega|-| \omega_{\text {analy }}|| /\left|\omega_{\text {analy }}\right|$ of the absolute value of the numerically obtained eigenfrequency $|\omega|$ to the (semi-)analytical reference value $\left|\omega_{\text {analy }}\right|$ as a function of the normalized step size $\Delta / \Delta_{0}$ for the 10 lowest TM-modes for a biased, lossy, rectangular cavity resonator filled with both gyromagnetic and gyroelectric material with electric boundary conditions. [59] 


\section{Applications}

In this chapter, the newly developed solver mentioned in chapter 3 is employed for the simulation of two different cavities loaded with the Ferroxcube $8 \mathrm{C} 12 \mathrm{~m}$ material taking into account the measured magnetic characteristics (cf. section 2.3). The first one is the setup used for the reflection measurement described in section 2.3. Since precise measurement data for the resonance frequency for different bias currents have been recorded, the comparison with simulation results serves as a further verification. Thereafter, a realistic model of the GSI SIS 18 cavity is considered. Obtained simulations for the fundamental mode are compared to available measured data and to results from calculations employing nonlinear equivalent circuit models. Moreover, to show the further potential of the solver, also higher-order modes are investigated and an outlook on potential 2-directional bias schemes is given.

Since the resonators of both application examples are filled with same ferrite material, the common prerequisites for the incorporation of the material data are given in section 4.1. The simulation of the two cavities is then discussed in sections 4.2 and 4.3. The main parts presented in this chapter are also published in [30].

\subsection{Prerequisites for the material modeling}

According to the computational model introduced in section 3.2, the field distribution of the magnetic field excited by the bias current is calculated in a first step. Here, the nonlinear characteristic equation (2.73) with the set of parameters given in subsection 2.3.7 as obtained from measurements is taken into account. Though the solver for the subsequent solution of the eigenvalue problem is capable of handling gyrotropic materials, the characteristics of the ferrite material are only modeled with a complex scalar isotropic permeability. This is due to the restriction that only information regarding the $\mu_{z}$-component of the permeability tensor can be retrieved from the measurement data. The local values of the $\mu_{z}$-component in each mesh cell are calculated by linear interpolation from a two-dimensional lookup table, which contains all the smoothed permeability values acquired from the analysis of the reflection measurement as a function of the bias magnetic field and frequency. The relative permittivity of the ferrite is assumed to be 25 , which is the estimated value for a frequency of $1 \mathrm{MHz}$ stated in the data sheet [41]. In fact, as is confirmed by simulations, its precise value is not of importance for the investigated fundamental eigenmode since it exhibits a very small electric field strength in the ferrite material.

\subsection{Simulation of the reflection measurement setup}

The computer-aided design (CAD) model for the simulation of the reflection measurement setup, which is described in subsection 2.3, is shown in figure 4.1. In order to accommodate for the additional parallel capacitance, a lumped element capacitor is inserted in the center, which is connected with thin perfectly conducting wires to the upper and lower plate of the cavity. 


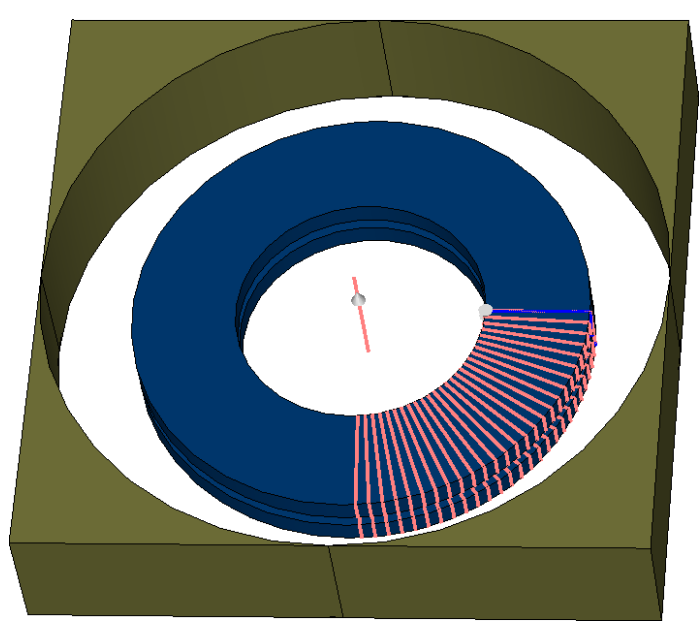

Figure 4.1.: CAD-model for the simulation of the reflection measurement setup. The ferrite toroids (blue) are located inside a PEC cavity (ocher). The perfectly conducting wire including the lumped element capacitor is seen in the center. Moreover, the bias current windings are only shown in one quarter.

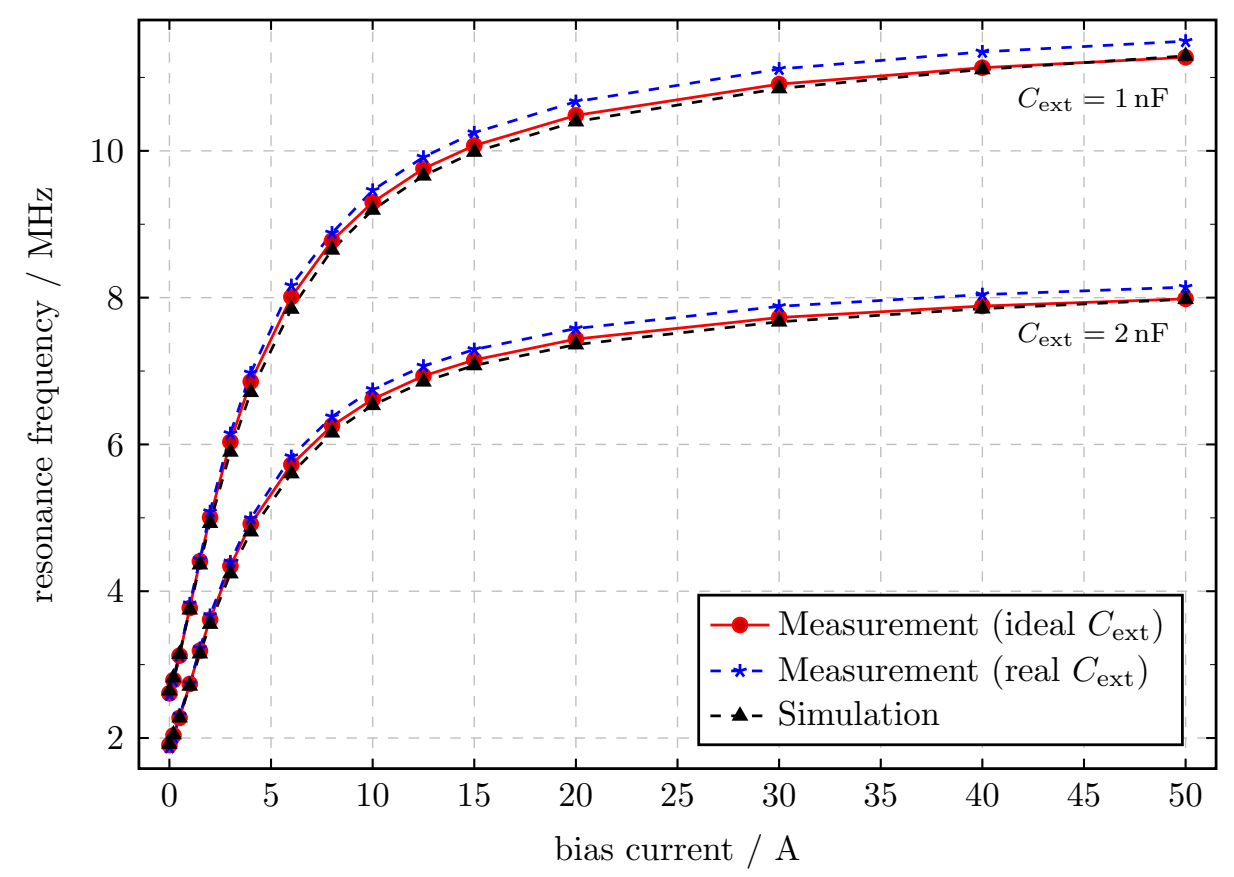

Figure 4.2.: Resonance frequency as a function of the bias current for the reflection measurement setup with external capacitors of $C_{\text {ext }}=1 \mathrm{nF}$ and $C_{\text {ext }}=2 \mathrm{nF}$, respectively. The values are shown as obtained from the measurement with a capacitor installed in parallel including the correction for its slight nonlinearity (real $C_{\text {ext }}$ ) and as obtained from the measurement data without external capacitor, which is modified as if an ideal capacitor was installed in parallel (ideal $C_{\text {ext }}$ ). Moreover, results obtained from the eigenmode simulation are included. 


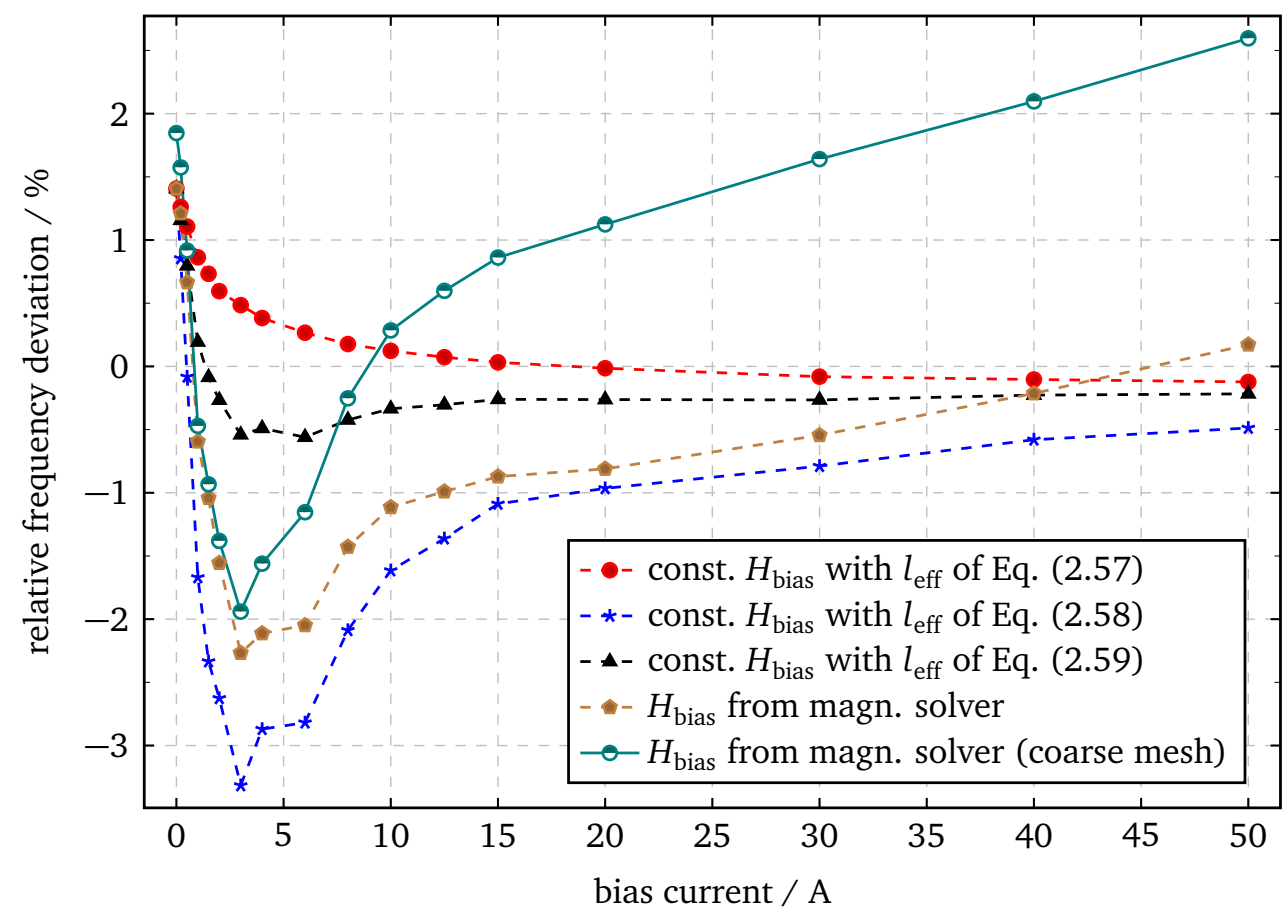

Figure 4.3.: Relative deviation of the resonance frequencies computed with the eigenmode simulation with different assumed bias magnetic field distributions compared to the resonance frequencies obtained from measurement modified for an ideal capacitor of $C_{\text {ext }}=1 \mathrm{nF}$. The following simulation results are compared: Eigenmode computations with a constant effective bias magnetic field assuming an effective magnetic path length given in equations (2.57), (2.58) and (2.59) as well as one where the field distribution is calculated with the magnetostatic solver (for all with $1.1 \times 10^{6}$ DOFs for one quarter). To estimate the general accuracy of the numerical calculation also the relative deviation of the simulation with the magnetostatic solver for $0.42 \times 10^{6}$ DOFs for one quarter is included.

Besides this exception for the centric wire all dimensions of the cavity and the ring cores are set to the measured values (cf. Fig. 2.7). Due to the symmetry of the setup and the electromagnetic field distribution of the fundamental mode, the field solutions are only calculated in one quarter of the setup using $1.1 \times 10^{6}$ DOFs. The numerical computation is performed for external parallel capacitors with capacitances of $C_{\text {ext }}=1 \mathrm{nF}$ and $C_{\text {ext }}=2 \mathrm{nF}$, for which also measurements were performed. The calculated eigenfrequencies are compared to the measured lowest resonances, which are found at those frequencies where the susceptance, i.e. the imaginary part of $Y$, vanishes. For a more reliable comparison with the simulation results, the measured data are corrected for the slightly nonlinear frequency characteristic of the capacitors as explained in subsection 2.3.2.1. Despite that correction there is a significant deviation between the measured values and the simulation (cf. measurement with real $C_{\text {ext }}$ in Fig. 4.2). Hence, the values are also compared with data measured without external capacitor and thereafter modified in a way as if an ideal capacitance of $C_{\text {ext }}=1 \mathrm{nF}$ and $C_{\text {ext }}=2 \mathrm{nF}$, respectively, was installed. This can simply be achieved by subtracting i $\omega C_{\text {ext }}$ from the measured admittance. Figure 4.2 shows the dependence of the resonance frequency on the bias magnetic field strength as obtained from measurement and numerical computation for the two capacitances. 


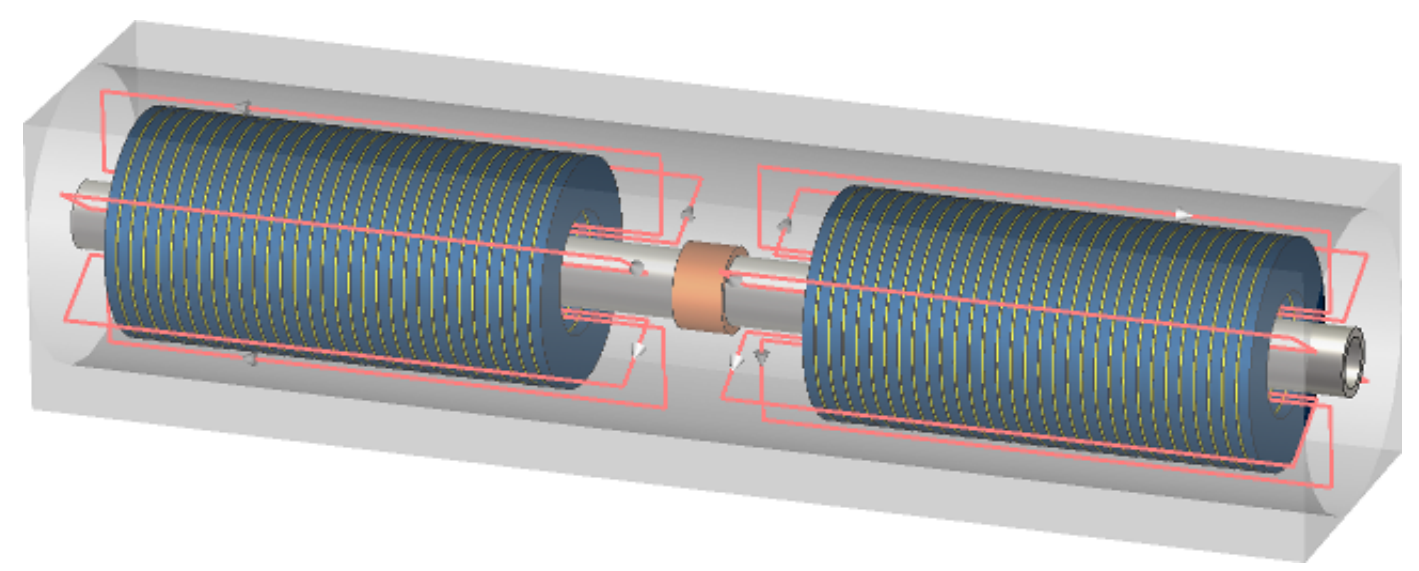

Figure 4.4.: CAD-model used for the numerical simulation of the GSI SIS 18 cavity. The ferrite ring cores are shown in blue, the copper discs in yellow. The lumped element gap capacitors are not shown. [30]

As mentioned in subsection 2.3.2.1, different approaches for the calculation of the effective magnetic path length, which enters in the spatially constant effective bias magnetic field strength (2.56), have been established. With the help of the eigenmode simulation, a comparison of the three different choices with the measurement result on the one hand and with the simulation where the bias magnetic field is obtained from the solution of the magnetostatic field problem on the other hand is made. The effective magnetic path lengths for the dimensions of the ring cores are: $l_{\text {eff }}=1.139 \mathrm{~m}$ for equation (2.57), $l_{\text {eff }}=1.210 \mathrm{~m}$ for equation (2.58) and $l_{\text {eff }}=1.156 \mathrm{~m}$ for equation (2.59). Since the values of $l_{\text {eff }}$ differ only slightly from each other, this is also the case for the computed eigenfrequencies as shown in figure 4.3.

\subsection{Simulation of the GSI SIS 18 ferrite cavity}

The model used in the numerical simulation of the GSI SIS 18 cavity is depicted in figure 4.4. On each side of the accelerating gap 32 ferrite ring cores are installed. Between these ferrite cores, copper cooling discs are present, which are modeled as PEC material. Additionally, the aluminium-oxide ceramic around the gap is taken into account with a relative permittivity of 9.5 and an electrical loss factor of $10^{-4}$. Moreover, four gap capacitors are included as lumped element capacitors. Adopting the results of the analysis in [64], their total capacitance is set to $C_{\text {tot }}=640 \mathrm{pF}$. Note, however, that this value is subject to unknown, potentially large uncertainties due to the difficulties in estimating the distributed capacitances of the resonator system.

\subsubsection{Analysis of the fundamental mode}

The fundamental mode exhibits electric field components longitudinal to the beam axis in the gap area and can be used for acceleration of the charged particles. The magnetic induction is accordingly oriented azimuthally in the ferrite ring cores and hence parallel to the bias field. Employing the symmetry of the CAD-model and of the fundamental mode, it is sufficient to perform the simulation in only one quarter of the cavity. The results of the eigenmode simulation presented in the following were computed with $2.5 \times 10^{6} \mathrm{DOFs}$ for the remaining quarter of the 


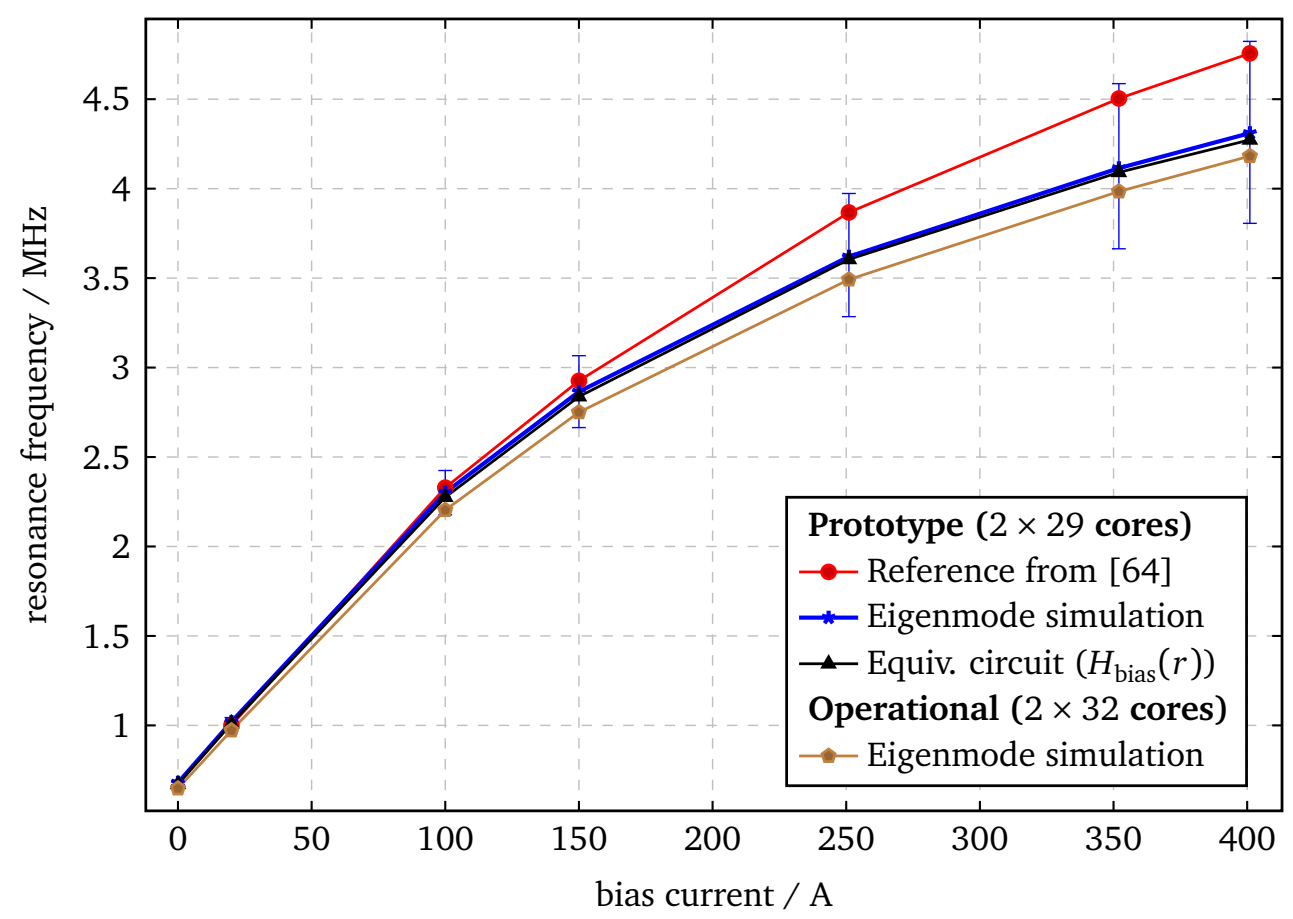

Figure 4.5.: Resonance frequency as a function of the bias current for the prototype cavity with 29 cores on each side of the gap for small RF-levels. The reference values are taken from table 3.3 of [64]. The simulation results are shown for the values of the permeability as obtained from the reflection measurements including error bars, which are computed in separate computations for $\mu \pm 0.5 \times \Delta \mu$, where $\Delta \mu$ is the uncertainty as estimated from the error analysis of the measurement data. Moreover, resonance frequencies calculated with the equivalent circuit model taking into account the radial dependence of the bias field are included. For completeness, the results of the eigenmode simulation are also added for the operational cavity with two times 32 toroids. [30]

computational model. The dependence of the resonance frequency on the bias current is shown in figure 4.5. For the estimation of its uncertainty, the computations were repeated for values of the permeability of $\mu \pm 0.5 \times \Delta \mu$, where $\mu$ is the best estimate and $\Delta \mu$ the estimated uncertainty of the permeability (cf. subsection 2.3.3). Furthermore, to estimate the discretization error, simulations were performed on different meshes. A refinement from $\approx 0.3 \times 10^{6} \mathrm{DOFs}$ to $2.5 \times 10^{6}$ DOFs revealed relative deviations in the order of $1 \%$. It is hence concluded that the discretization error is negligible compared to the material uncertainty. What is more, since reference values are only available for a special setup of the SIS 18 prototype cavity, which effectively contains only 29 ferrite toroids on each side of the gap, the simulations are also performed for the reduced number of ring cores to allow a more reliable comparison. As is evident from figure 4.5 and 4.6, the simulation results are in accordance with measurement data [64] within an error range of approximately $10 \%$ albeit the latter were recorded for moderate RF-levels. Furthermore, the obtained quality factor $Q=\operatorname{Re}(\omega) /(2 \operatorname{Im}(\omega))$ for the complex resonance frequency $\omega$ as a function of the bias current is depicted in figure 4.7, again for small RF-levels. Since the numerical simulation reveals the unloaded quality factor, a few available reference values for the loaded cavity and tetrode are thus accordingly transformed [64]. Nevertheless, those results are only comparable to a limited extent. 


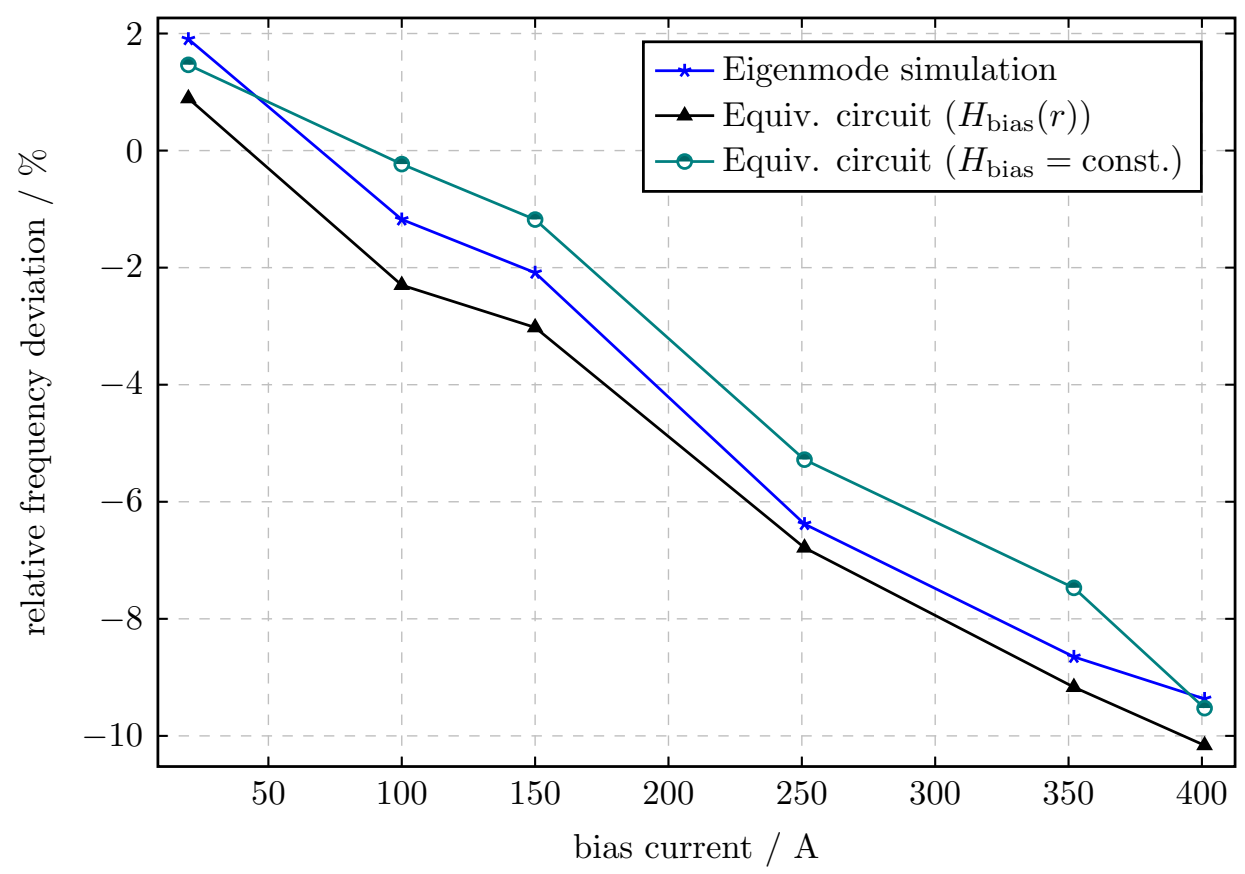

Figure 4.6.: Relative deviation of the resonance frequency obtained from simulation to the reference values as a function of the bias current for the prototype cavity with 29 cores on each side of the gap. The comparison includes values from the eigenmode simulation and the calculation using the equivalent circuit model for the simplified assumption of an effective mean magnetic bias field according to equations (2.56) and (2.57) as well as the model in which the radial dependence of the bias field is taken into account. [30] 


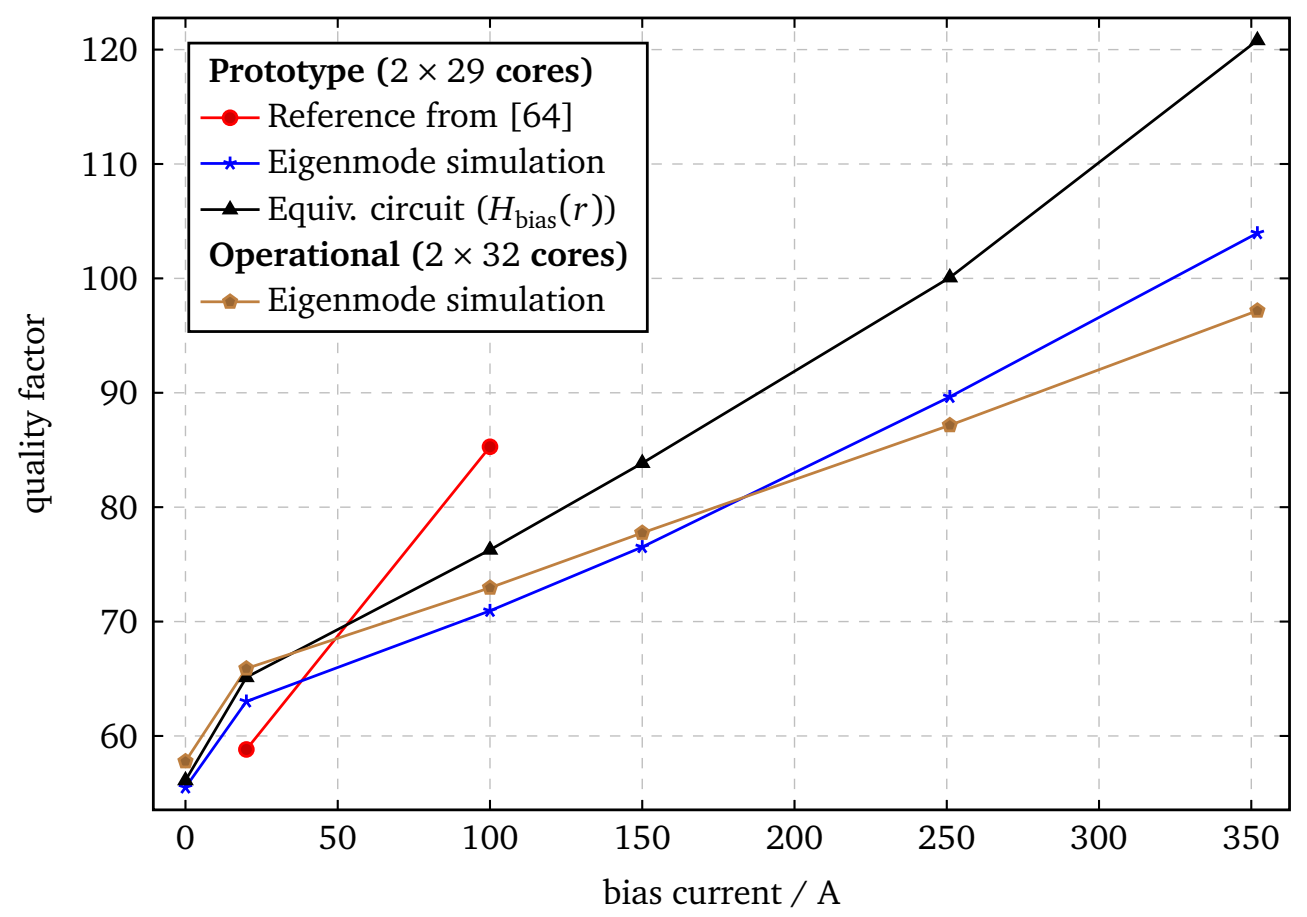

Figure 4.7.: Quality factor as a function of the bias current for the prototype cavity with 29 cores on each side of the gap for small RF-levels. The reference values are calculated from [64]. The simulation results are shown for the values of the permeability as obtained from the reflection measurements. Moreover, the quality factor based on an equivalent circuit model taking into account the radial dependence of the bias field is also included. For completeness, the results of the eigenmode simulation are supplementary added for the operational cavity with two times 32 toroids. [30]

Finally, it is confirmed by simulations that the effect of the copper cooling discs is fully negligible with respect to the resonance frequency for the fundamental mode. The same results are even observed when the ferrite ring cores are modeled as only one contiguous toroid with the thickness of the sum of the individual cores. This could be exploited to reduce the DOFs and thereby further speed up the computation.

\subsubsection{Comparison with nonlinear equivalent circuit model}

The calculation of the resonance frequency and quality factor for the fundamental mode is also performed with the help of an equivalent circuit model as depicted in figure 4.8. The resonance frequency of this circuit is implicitly given by

$$
f_{\text {res }}=\frac{1}{2 \pi} \sqrt{\frac{1}{\left(C_{\text {tot }}+C_{\text {gap }}\right) L_{\mathrm{s}}\left(f_{\text {res }}, I_{\text {bias }}\right)}-\left[\frac{R_{\mathrm{s}}\left(f_{\text {res }}, I_{\text {bias }}\right)}{L_{\mathrm{s}}\left(f_{\text {res }}, I_{\text {bias }}\right)}\right]^{2}} .
$$




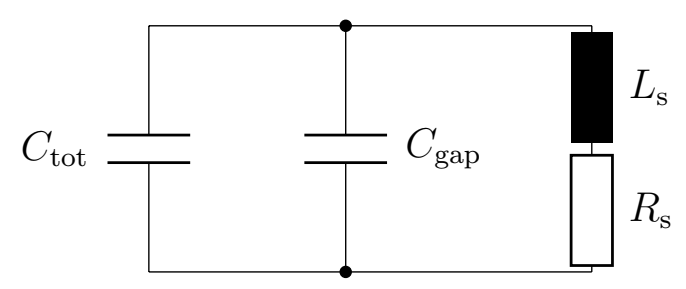

Figure 4.8.: Equivalent circuit model for the calculation of the fundamental mode of the GSI SIS 18 cavity setup. In addition to the total capacitance $C_{\text {tot }}=640 \mathrm{nF}$, which is also included in the eigenmode computation as lumped element capacitors, the gap capacitance $C_{\text {gap }}=30 \mathrm{nF}$ is installed in parallel. The ferrite ring cores are modeled as a series inductance $L_{\mathrm{s}}$ and series resistance $R_{\mathrm{s}}$. [30]

To account for the nonlinear dependence of the permeability on the frequency and on the bias magnetic field strength, the series inductance $L_{\mathrm{s}}$ and series resistance $R_{\mathrm{s}}$ are calculated according to the relations (cf. subsection 2.3.2.1)

$$
\begin{aligned}
& L_{\mathrm{s}}\left(f, I_{\text {bias }}\right)=N_{\text {cores }} h_{\text {core }} \int_{r_{\text {in }}}^{r_{\text {out }}} \frac{\mu_{\mathrm{s}}^{\prime}\left[f, H\left(I_{\text {bias }}\right)\right]}{2 \pi r} \mathrm{~d} r+L_{\text {air }}, \\
& R_{\mathrm{s}}\left(f, I_{\text {bias }}\right)=2 \pi f N_{\text {cores }} h_{\text {core }} \int_{r_{\text {in }}}^{r_{\text {out }}} \frac{\mu_{\mathrm{s}}^{\prime \prime}\left[f, H\left(I_{\text {bias }}\right)\right]}{2 \pi r} \mathrm{~d} r
\end{aligned}
$$

with the inside length of the cavity $l_{\text {cav }}=2.85 \mathrm{~m}$, the number of ring cores $N_{\text {cores }}$ and the inductance of the empty cavity including the copper discs of height $h_{\text {copper }}$

$$
L_{\text {air }}=\frac{1}{2 \pi} l_{\text {cav }} \mu_{0} \ln \frac{r_{\text {cav,out }}}{r_{\text {cav,in }}}-\frac{1}{2 \pi} 2\left(N_{\text {cores }}-1\right) h_{\text {copper }} \mu_{0} \ln \frac{r_{\text {out }}}{r_{\text {in }}}=793 \mathrm{nH} .
$$

As for the eigenmode computation, the values of the real and imaginary part of the permeability are obtained with linear interpolation from the two-dimensional lookup table. For the bias magnetic field strength $H\left(I_{\text {bias }}\right)$ either the expression involving the effective magnetic path length (2.56) or the assumed analytical dependence

$$
H\left(I_{\text {bias }}\right)=\frac{N_{\text {bias }} I_{\text {bias }}}{2 \pi r}
$$

is inserted. In the former case, the integral in equation (4.2) can be evaluated analytically; otherwise it is solved by numerical integration. In both cases the roots of equation (4.1) are found by standard numerical algorithms without difficulties. The obtained results for the resonance frequency as well as the quality factor of the fundamental mode of the prototype cavity are included in figure 4.5, 4.6 and 4.7.

\subsubsection{Analysis of higher-order modes}

Unlike the simple equivalent circuit model, the eigenmode computation also allows for the analysis of higher-order modes (HOMs). To ensure that no HOM is omitted, the whole cavity 
is discretized without usage of any symmetry plane. Three different types of HOMs are found: The type with lowest frequencies is characterized by a perpendicular electric field between the beam pipe and the ferrite cores. The frequency strongly depends on the permeability of the toroids. Thus, the lowest resonance frequency of this type is found at $\approx 3 \mathrm{MHz}$ in the remanence state and increases up to $\approx 23 \mathrm{MHz}$ for $I_{\text {bias }}=400 \mathrm{~A}$. For the second type, the electric field resides inside the ferrite toroids between the copper discs. In fact, there is a whole spectrum of such modes for all possible combinations of relative orientations of the electric field from one core to adjacent ones. Besides from the dependence on the permittivity of the ferrite, also its permeability plays a crucial role for their resonance frequencies. Consequently, HOMs of this type are found with frequencies above $\approx 7 \mathrm{MHz}$ for a bias current of $I_{\text {bias }}=20 \mathrm{~A}$ but do not show up below $\approx 30 \mathrm{MHz}$ for $I_{\text {bias }}>400 \mathrm{~A}$. Of course, the stated resonance frequencies must be perceived as rough estimates only, due to the very high uncertainty of the permeability for large frequencies. Finally, the third type represents a dipole mode with strong electric field components in the accelerating gap. Its resonance frequency at $\approx 39 \mathrm{MHz}$ is far above the fundamental mode. Moreover, this mode is independent of the permeability of the ferrite rings and thus independent of the bias current. The three types have in common that they exhibit mainly a magnetic field parallel to the bias field inside the ferrite cores. It is recalled that the material data are available only for such parallel biasing. Thus, it cannot be excluded from the performed analysis that there are additionally HOMs perpendicular to the magnetic bias field within the investigated frequency range.

\subsubsection{2-directional bias scheme}

With the aim of showing the further potential of the eigenmode solver, possible schemes with a superposition of a parallel and a perpendicular bias magnetic field, so-called 2-directional bias schemes, are examined. As expected from theory [102], such bias schemes might lead to a higher quality factor while sustaining the same tuning range. This fact has been successfully confirmed in recent experiments [39]. A numerical analysis of a 2-directional bias scheme requires the knowledge of all components of the permeability tensor (2.22) for all magnetization states. As, however, only the $\mu_{z}$-component is available from measurements, a theoretical model is applied to estimate the missing components utilizing the parameters $\alpha$ and $H_{a}$. These parameters have been found in section 2.3.6.1 with the help of a proper fit to the available $\mu_{z}$-component. Two different models are tested. In the first one, it is assumed that the diagonal component $\widetilde{\mu}_{\text {diag }}$ can be described by equation (2.28a) and the off-diagonal component by [21]

$$
\widetilde{\kappa}=\kappa \frac{M_{z}}{M_{\text {sat }}} \times \frac{\widetilde{\mu}_{\text {diag }}}{\mu_{\text {diag }}}
$$

with $\mu_{\text {diag }}$ and $\kappa$ from the fully magnetized state (cf. Eq. (2.23)). The second model is the one of Igarashi and Naito (cf. subsection 2.2.3.3), i.e. with the tensor as given in equation (2.31). From this model also the relation for $\tilde{\mu}_{z}$ was obtained (cf. subsection 2.3.6.1). For consistency of the main diagonal elements, the expression for $\widetilde{\mu}_{\text {diag }}$ is, however, modified in the same way as it was done for $\widetilde{\mu}_{z}$. It then takes the form:

$$
\tilde{\mu}_{\text {diag }}=1+\frac{1}{2}(\hat{\mu}-1)\left(1+\left(\frac{M_{z}}{M_{\text {sat }}}\right)^{1.15}\right) .
$$


resonance frequency / $\mathrm{MHz}$

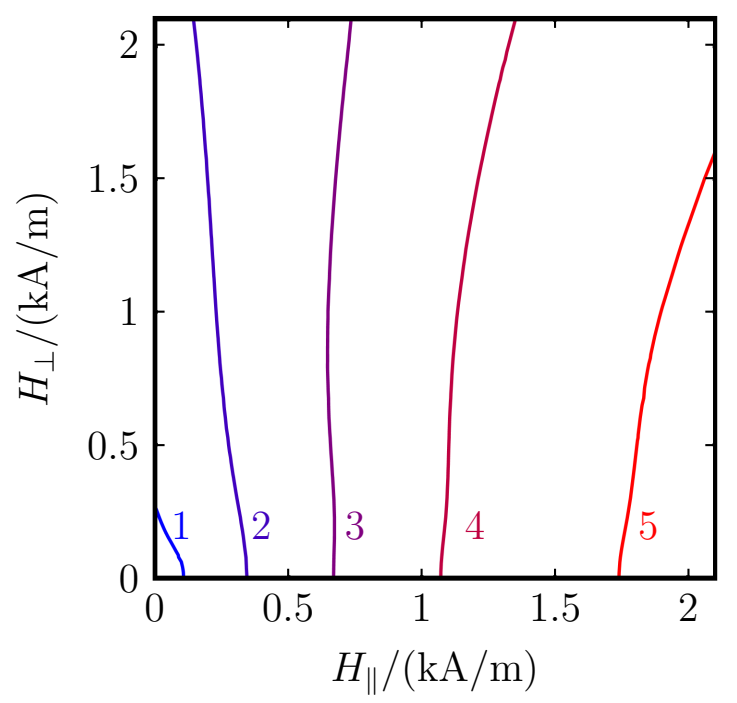

quality factor

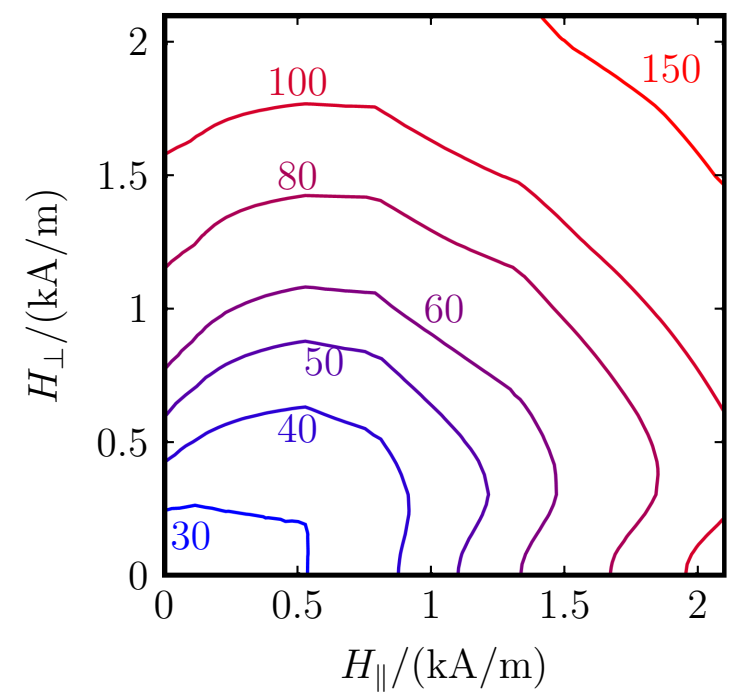

Figure 4.9.: Resonance frequency (left) and quality factor (right) of the fundamental mode obtained from numerical simulations with the modified material model as specified in equation (2.70) and (4.6) as a function of the parallel and perpendicular bias magnetic field strength $H_{\|}$and $H_{\perp}$, respectively. [30]

It is further assumed that the anisotropy field $H_{a}$ acts in the direction of parallel bias. Assuming these characteristics, eigenmode computations of the fundamental mode of the GSI SIS 18 cavity are performed. Here, a sweep over different values for the parallel and perpendicular bias magnetic field strength is performed. The parallel bias field is obtained from the solution of the magnetostatic problem as for the standard SIS 18 cavity whereas for the perpendicular one a spatially constant homogeneous field is added. Since it was found for purely parallel biasing that the copper discs do not significantly affect the results (cf. subsection 4.3.1), the following simulations are accomplished without copper discs for efficiency. Whereas there is no indication for a possible loss reduction by means of a 2-directional bias-scheme for the first choice of parameters, promising results are obtained for the second material model. Here, the additional perpendicular field hardly changes the resonance frequency in a wide range. Yet, the quality factor strongly depends on both field components. Consequently, this model is compliant with the experimental finding that a 2-directional bias scheme is beneficial [39]. The results for the resonance frequency and quality factor are summarized in figure 4.9.

Clearly, several assumptions led to the approximation of the properties of the Ferroxcube $8 \mathrm{C} 12 \mathrm{~m}$ ferrite with the above terms. Hence, further measurements to determine all components of the permeability tensor are desirable. 


\section{Summary and outlook}

To sum up, the eigenmode computation of cavity resonators filled with biased gyrotropic materials has been elaborated in this thesis. In this regard, the following aspects have been discussed.

Eigenvalue problems with gyromagnetic substances emerge in the field of accelerator physics for the analysis of ferrite-loaded cavities. As reviewed in the thesis at hand, the fundamental magnetic properties of ferrites are similar to those of ferromagnetic materials including the presence of a spontaneous magnetization with all its consequences such as the development of magnetic domains and a nonlinear $B-H$ dependence. What is more, the gyromagnetic characteristics of ferrites can be described by a permeability tensor whose components are a function of the frequency of the superimposed RF as well as the bias magnetic field. Due to the lack of material data for the Ferroxcube $8 \mathrm{C} 12 \mathrm{~m}$ ferrite, which are required for the eigenmode simulation of the GSI SIS 18 cavities, goal-oriented measurements were performed at the GSI facility. The complex permeability is determined in two different approaches, one in a reflection and one in a transmission setup. The obtained values for low RF-levels are fully compatible with each other within their estimated error margins up to moderate frequencies. Beyond that the transmission approach is not applicable anymore due to $L C$-resonances. Moreover, an analytical relation for the real and imaginary part of the permeability is found empirically, which properly describes the dependence on the bias magnetic field strength and the frequency particularly in the low frequency range. Moreover, also the $B-H$ curve which is enclosed in a reduced working cycle of the cavity was recorded. Though ferrites do not exhibit gyroelectric properties, also the permittivity tensor, which is relevant for the description of gyroelectric substances such as magnetized plasmas, has been presented.

As pointed out, the need for a dedicated solver for the calculation of eigenmodes of resonators filled with gyrotropic substances arises from their challenging material properties. According to the proposed computational model, the field problem is thus divided into two parts: firstly, a magnetostatic problem for the calculation of the magnetic field excited by the bias current to be able to evaluate the material properties locally at the specified working point; secondly, a nonlinear and, if material losses are taken into account, also non-Hermitian eigenvalue problem. For their numerical solution, the FIT with a hexaedral staircase filling is employed in this work. To this end, the standard formulation has been extended to gyromagnetic as well as gyroelectric materials in frequency domain. The derived expressions for the non-diagonal inverse permeability and permittivity matrices reduce to diagonal form of the standard FIT if no gyrotropic materials are present. Furthermore, due to the averaging of the material properties by taking into account the partial length or area of the dual edges and facets, respectively, the material matrices feature a manifestly Hermitian structure in the absence of material losses, even in the case of non-equidistant grids. In the description of the FIT in this thesis, the focus is particularly put on different ordering schemes of the FIT-DOFs including also remarks on a fully general ordering. The actual implementation is based on a scheme which is well suited for a distributed computation. Moreover, an extensive discussion on the reduced grid, in which all a priori vanishing DOFs are completely removed, is given. Thanks to these and other elaborated aspects affecting efficiency, the implemented solver is capable of solving gyrotropic eigenprob- 
lems discretized with several millions of mesh cells in a reasonable time. To confirm the validity of the numerically obtained results, the nonlinear magnetostatic solver and the component for the solution of nonlinear, non-Hermitian eigenproblems is confirmed by thorough comparisons with (semi-)analytical calculations.

To demonstrate that the newly developed solver can be used in practical applications, the data for the scalar, isotropic permeability retrieved from the mentioned measurements are incorporated into the computational model. For its verification, the measurement setup is successfully simulated at first. After that, a realistic model of the GSI SIS 18 cavity is considered. The obtained values for the resonance frequency and quality factor for the fundamental mode are in accordance with available measurement data. Moreover, it is revealed that the analysis of the fundamental mode of ferrite-loaded cavity resonators by means of a nonlinear equivalent circuit model provides almost identical results as the computationally by far more expensive eigenmode simulation. However, provided that material data of the full permeability tensor are available, it is shown that the eigenmode computation can also help to investigate possibly advantageous 2-directional bias schemes.

In conclusion, the developed solver provides means to efficiently analyze cavities filled with biased gyrotropic materials taking into account aspects such as the dependence of the material on frequency and bias field as well as material losses. Currently, the accuracy of the obtained results is only limited by the quality of available material data. Hence, further measurements to precisely determine all components of the material tensors are desirable. Regarding the numerical solution, it might be interesting to extend the presented expressions for the gyrotropic material matrices to the Conformal Finite Integration Technique [103], as accomplished for diagonal matrices in [67], to overcome the restriction of staircase fillings. 


\section{A Proofs}

\section{A.1 Proof of the properties of the topological matrices for arbitrary ordering schemes}

In subsection 3.4.5.4 relations between topological matrices of the primary and dual grid are discussed, which are directly deduced there only for the special cases of ordering scheme 1 (cf. subsection 3.4.5.1) and 2 (cf. subsection 3.4.5.2). In this section the missing proof that these properties still hold for any arbitrary ordering scheme is supplied. Only the proof for the relation between the matrices $\mathbf{C}$ and $\widetilde{\mathbf{C}}$ (cf. Eq. (3.72)) is given explicitly since the ones for all other relations are completely analogous.

Any general ordering of the FIT-DOFs can be obtained out of a given ordering, say, e.g., ordering 1 , by permuting the elements of the given scheme. More precisely, given a vector $\mathbf{v}_{1}$ with the DOFs arranged as in ordering scheme 1 , the vector $\mathbf{v}_{A}$ for any general ordering scheme $A$ is the result of the product of a permutation matrix $P_{\pi}$ with the vector $\mathbf{v}_{1}$. Moreover, since any permutation can be decomposed into the product of transpositions ${ }^{1} \mathscr{P}$, the vector of ordering $A$ can be calculated as

$$
\mathbf{v}_{1} \rightarrow \mathbf{v}_{A}=P_{\pi} \mathbf{v}_{1}=\prod_{i=1}^{k} \mathscr{P}_{i} \mathbf{v}_{1}=\mathscr{P}_{1} \mathscr{P}_{2} \ldots \mathscr{P}_{k} \mathbf{v}_{1}
$$

As pointed out in section 3.4.5, also the structure of the topological matrices has to be adjusted. Since the rows of the matrix $\mathbf{C}$ behave as $E$-field-like vectors and columns as $H$-field-like vectors and vice versa for the matrix $\widetilde{\mathrm{C}}$ (cf. subsection 3.4.7.1), their rows and columns transform accordingly. Hence, starting from the given matrices $\mathbf{C}_{1}$ and $\widetilde{\mathbf{C}}_{1}$ for ordering one, the transformed matrices take the form

$$
\begin{aligned}
\mathrm{C}_{1} & \rightarrow \mathrm{C}_{A}=\mathscr{P}_{1}^{\mathrm{E}} \mathscr{P}_{2}^{\mathrm{E}} \ldots \mathscr{P}_{l-1}^{\mathrm{E}} \mathscr{P}_{k}^{\mathrm{E}} \mathbf{C}_{1} \mathscr{P}_{1}^{\mathrm{H}} \mathscr{P}_{2}^{\mathrm{H}} \ldots \mathscr{P}_{l-1}^{\mathrm{H}} \mathscr{P}_{l}^{\mathrm{H}}, \\
\widetilde{\mathbf{C}}_{1} & \rightarrow \widetilde{\mathbf{C}}_{A}=\mathscr{P}_{l}^{\mathrm{H}} \mathscr{P}_{l-1}^{\mathrm{H}} \ldots \mathscr{P}_{2}^{\mathrm{H}} \mathscr{P}_{1}^{\mathrm{H}} \widetilde{\mathbf{C}}_{1} \mathscr{P}_{k}^{\mathrm{E}} \mathscr{P}_{k-1}^{\mathrm{E}} \ldots \mathscr{P}_{2}^{\mathrm{E}} \quad \mathscr{P}_{1}^{\mathrm{E}},
\end{aligned}
$$

where the permutations for $H$-field- and $E$-field-like vectors are the composition of the transpositions $\mathscr{P}_{i}^{\mathrm{H}}$ and $\mathscr{P}_{i}^{\mathrm{E}}$, respectively. Since transpositions are symmetric, i.e.

$$
\mathscr{P}_{i}=\mathscr{P}_{i}^{T},
$$

from equations (A.2) the desired result

$$
\begin{aligned}
& \mathbf{C}_{A}^{T}=\left(\mathscr{P}_{1}^{\mathrm{H}} \mathscr{P}_{2}^{\mathrm{H}} \ldots \mathscr{P}_{l-1}^{\mathrm{H}} \mathscr{P}_{l}^{\mathrm{H}}\right)^{T} \mathbf{C}_{1}^{T}\left(\mathscr{P}_{1}^{\mathrm{E}} \mathscr{P}_{2}^{\mathrm{E}} \ldots \mathscr{P}_{k-1}^{\mathrm{E}} \mathscr{P}_{k}^{\mathrm{E}}\right)^{T}= \\
& =\mathscr{P}_{l}^{\mathrm{H}} \mathscr{P}_{l-1}^{\mathrm{H}} \ldots \mathscr{P}_{2}^{\mathrm{H}} \mathscr{P}_{1}^{\mathrm{H}} \widetilde{\mathbf{C}}_{1} \mathscr{P}_{k}^{\mathrm{E}} \mathscr{P}_{k-1}^{\mathrm{E}} \ldots \mathscr{P}_{2}^{\mathrm{E}} \mathscr{P}_{1}^{\mathrm{E}}=\widetilde{\mathbf{C}}_{A}
\end{aligned}
$$

follows immediately provided that $\mathbf{C}_{1}^{T}=\widetilde{\mathbf{C}}_{1}$.

$\overline{1}$ A transposition is a permutation which swaps exactly two elements whereas all others stay unchanged. 

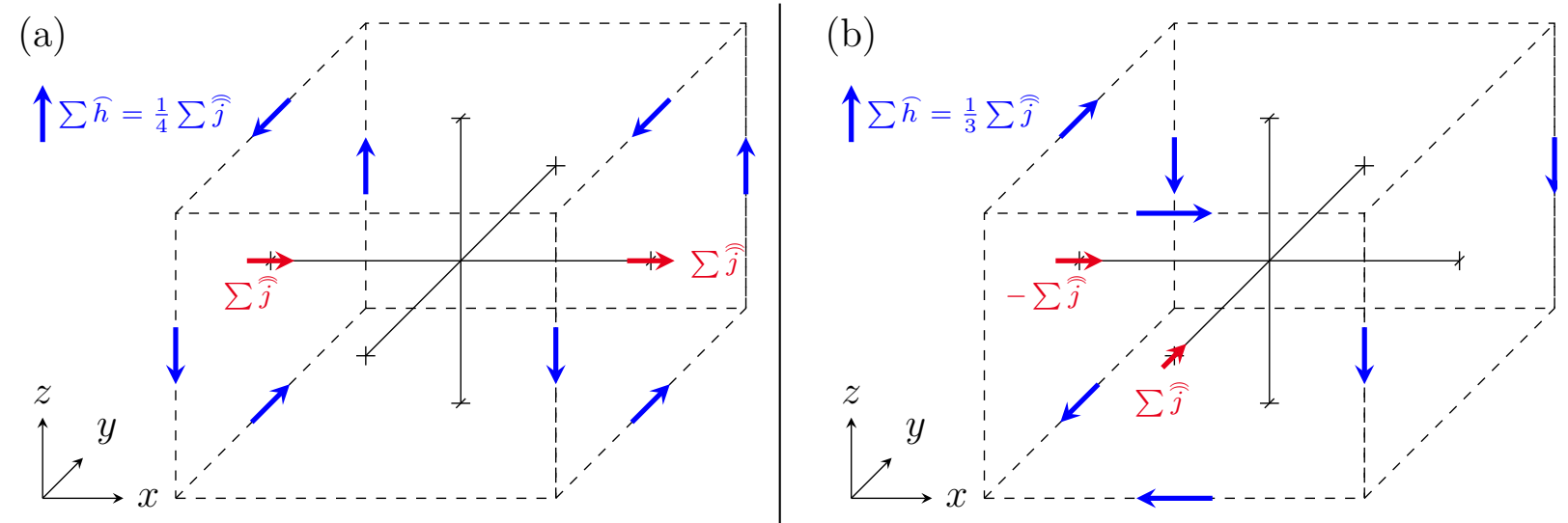

Figure A.1.: Elements of the $\widehat{\mathbf{h}}_{i}$-field due to a symmetric current going in $x$-direction (a) and an asymmetric current entering in positive $y$-direction and leaving in negative $x$ direction (b). The sum of the $\widehat{h}_{i}$-components along a dual edge is set with the sign as indicated by the direction of the arrow. On edges where no arrow is present, no values are set.

\section{A.2 $H_{i}$-algorithm}

In this subsection, a proof is given that the $H_{i}$-algorithm, which is presented in subsection 3.4.11.2, results in a magnetic field $\widehat{\mathbf{h}}_{i}$ which satisfies the rotational equation (3.130b). In the first subsection, the proof is provided only for the edges along the surfaces of the initial volume, i.e. for step 2 of the algorithm. In the subsequent subsection, it is shown that the way how the components of the $\widehat{\mathbf{h}}_{i}$-field are determined after each subdivision in step 3 ensures that the rotational equation is fulfilled everywhere, eventually.

\section{A.2.1 Initialization of the $H_{i}$-components on the surface}

In the special case of closed current paths inside the computation domain, which do not go through its surface, the rotational equation can be fulfilled trivially on the surface by setting all $\widehat{\mathbf{h}}_{i}$-components on the surface edges to zero. If, however, current flows through the surface the following strategy can be pursued (for notation is referred to Fig. 3.11): First of all, according to the continuity equation the sum of currents that enter the volume must equal the sum of currents that leave the volume, i.e.

$$
\sum \widehat{\hat{j}}_{a}+\sum \widehat{\hat{j}}_{e}+\sum \widehat{\hat{j}}_{c}-\sum \widehat{\hat{j}}_{b}-\sum \widehat{\hat{j}}_{d}-\sum \widehat{\hat{j}}_{f}=0 .
$$

The idea is then to decompose the surface currents into symmetric and asymmetric contributions. In this context the symmetric part is understood as current which enters and leaves the volume in the same coordinate direction; the asymmetric one enters and leaves the volume in a changed direction. As is shown in figure A.1, the field components can readily be found for such individual contributions. Employing this, firstly the $\widehat{\mathbf{h}}_{i}$-components due to a symmetric current 
$\sum \widehat{\bar{j}}_{b}$ both through the left and right surface in $x$-direction (cf. Fig. A.1(a)) are set, that is to say, the components are set as

$$
\begin{array}{ll}
\sum \widehat{h}_{n}=+\frac{1}{4} \sum \widehat{\hat{j}}_{b} & n=1,2,9,10 \\
\sum \widehat{h}_{n}=-\frac{1}{4} \sum \widehat{\hat{j}}_{b} & n=3,4,11,12 .
\end{array}
$$

Having done this, the current through the left surface must be set to $\widehat{\hat{j}}_{\Delta x}=\sum \widehat{\hat{j}}_{a}-\sum \widehat{\hat{j}}_{b}$ to correct for the made error, whereas no so far unconsidered current through the right surface remains. Secondly, the symmetric current in z-direction is considered. The $\widehat{\mathbf{h}}_{i}$-components due to current $\sum \widehat{\hat{j}}_{d}$ through both the lower and upper surface are set as

$$
\begin{array}{ll}
\sum \widehat{h}_{n}=+\frac{1}{4} \sum \widehat{\hat{j}}_{d} & n=5,7,9,11 \\
\sum \widehat{h}_{n}=-\frac{1}{4} \sum \widehat{\hat{j}}_{d} & n=1,3,6,8 .
\end{array}
$$

Analogously, the current through the lower surface must then be set to $\widehat{\hat{j}}_{\Delta z}=\sum \widehat{\hat{j}}_{c}-\sum \widehat{\hat{j}}_{d}$ to correct for the made error, whereas no current through the upper surface remains. Thirdly, the symmetric contribution due to a current $\sum \widehat{\hat{j}}_{f}$ in $y$-direction through the front and back surface is considered by setting the $\widehat{h}_{i}$-components as

$$
\begin{array}{ll}
\sum \widehat{h}_{n}=+\frac{1}{4} \sum \widehat{\hat{j}}_{f} & n=2,4,7,8 \\
\sum \widehat{h}_{n}=-\frac{1}{4} \sum \widehat{j}_{f} & n=12,5,10,6 .
\end{array}
$$

Again, the current through the front surface must then be set to $\widehat{\hat{j}}_{\Delta y}=\sum \widehat{\bar{j}}_{e}-\sum \widehat{\hat{j}}_{f}$ to correct for the made error. Yet, no current through the back surface is left unconsidered. Up to this point, only the asymmetric current contributions remain. Hence, fourthly, the $\widehat{\mathbf{h}}_{i}$-components due to an asymmetric current $\widehat{j}_{\Delta y}$ through the left and front surface in $x$ - and $y$-direction are set as (cf. Fig. A.1(b))

$$
\begin{array}{ll}
\sum \widehat{h}_{n}=+\frac{1}{3} \widehat{\hat{j}}_{\Delta y} & n=3,7 \\
\sum \widehat{h}_{n}=-\frac{1}{3} \widehat{\bar{j}}_{\Delta y} & n=1,2,5,10,12 .
\end{array}
$$

Afterwards, the current through the left surface must be set to $\widehat{\hat{j}}_{\Delta x, y}=\widehat{\hat{j}}_{\Delta x}+\widehat{\hat{j}}_{\Delta y}$ to correct for the made error, whereas no current through the front surface remains. Lastly, the $\widehat{\mathbf{h}}_{i}$-components due to an asymmetric current $\widehat{\hat{j}}_{\Delta z}$ both through the left and lower surface in $x$ - and z-direction are set as

$$
\begin{array}{ll}
\sum \widehat{h}_{n}=+\frac{1}{3} \widehat{\bar{j}}_{\Delta z} & n=3,4,5,9,11 \\
\sum \widehat{h}_{n}=-\frac{1}{3} \widehat{\hat{j}}_{\Delta z} & n=2,6 .
\end{array}
$$


After that, all current contributions have been considered since $\widehat{\hat{j}}_{\Delta x, y}+\widehat{\widehat{j}}_{\Delta z}=\widehat{\widehat{j}}_{\Delta x}+\widehat{\widehat{j}}_{\Delta y}+\widehat{\hat{j}}_{\Delta z}=$ 0 by virtue of the continuity equation (A.5). Thus, also no current through the left surface remains.

To sum up, all contributions due to the currents through the surfaces have been taken into account while satisfying the rotational equation (3.130b) by construction. The total of the set contributions on all twelve surface edges of the volume was already summarized in subsection 3.4.11.2. Of course, the above-mentioned solution is only one out of many possibilities.

\section{A.2.2 Determination of the $H_{i}$-components after each subdivision}

The proof that the way how the components of the $\widehat{\mathbf{h}}_{i}$-field are determined after each subdivision in subsection 3.4.11.2 finally generates a $\widehat{\mathbf{h}}_{i}$-field that fulfills the rotational equation (3.130b) is by induction. The aim is to show that, provided the rotational equation is already satisfied on all surfaces of a subvolume, it will be satisfied after the subdivision also on all new divided surfaces. It is sufficient to consider only one subdivision in $x$-direction without loss of generality. All relevant quantities referred to in the following are illustrated in figure 3.10.

The basis of the induction is that equation (3.130b) is already fulfilled on all the six surfaces of the subvolume. That is to say, the following holds:

$$
\begin{array}{rr}
\text { [front] } & \sum \widehat{h}_{9}+\sum \widehat{h}_{9}^{\prime}-\sum \widehat{h}_{5}-\sum \widehat{h}_{1}^{\prime}-\sum \widehat{h}_{1}+\sum \widehat{h}_{3}=\sum \widehat{\hat{j}}_{b}+\sum \widehat{\hat{j}}_{g} \\
\text { [bottom] } & \sum \widehat{h}_{1}+\sum \widehat{h}_{1}^{\prime}+\sum \widehat{h}_{4}-\sum \widehat{h}_{6}^{\prime}-\sum \widehat{h}_{6}-\sum \widehat{h}_{2}=\sum \widehat{j}_{a}+\sum \widehat{j}_{f} \\
\text { [left] } & \sum \widehat{h}_{7}-\sum \widehat{h}_{10}-\sum \widehat{h}_{3}+\sum \widehat{h}_{2}=\sum \widehat{\hat{j}}_{c} \\
\text { [right] } & \sum \widehat{h}_{8}-\sum \widehat{h}_{11}-\sum \widehat{h}_{5}+\sum \widehat{h}_{4}=\sum \widehat{j}_{j} \\
\text { [top] } & \sum \widehat{h}_{9}+\sum \widehat{h}_{9}^{\prime}+\sum \widehat{h}_{11}-\sum \widehat{h}_{12}^{\prime}-\sum \widehat{h}_{12}-\sum \widehat{h}_{10}=\sum \widehat{\widehat{j}}_{e}+\sum \widehat{\hat{j}}_{h} \\
\text { [back] } \sum \widehat{h}_{12}+\sum \widehat{h}_{12}^{\prime}-\sum \widehat{h}_{8}-\sum \widehat{h}_{6}^{\prime}-\sum \widehat{h}_{6}+\sum \widehat{h}_{7}=\sum \widehat{j}_{d}+\sum \widehat{j}_{i} .
\end{array}
$$

It is ensured in step 2 of the $H_{i}$-algorithm that the above assumptions hold true.

In the induction step, the subdivision in $x$-direction is performed. According to step 3 of the $H_{i}$-algorithm, the so far unknown $\widehat{h}_{i}$-components along the edges $13,14,15$ and 16 are determined as follows:

$\begin{aligned} \text { [front] } & \sum \widehat{h}_{14}=\sum \widehat{h}_{9}-\sum \widehat{h}_{1}+\sum \widehat{h}_{3}-\sum \widehat{j}_{b} \\ \text { [bottom] } & \sum \widehat{h}_{13}=-\sum \widehat{h}_{1}+\sum \widehat{h}_{6}+\sum \widehat{h}_{2}+\sum \widehat{\hat{j}}_{a} \\ \text { [top] } & \sum \widehat{h}_{16}=-\sum \widehat{h}_{9}+\sum \widehat{h}_{12}+\sum \widehat{h}_{10}+\sum \widehat{\hat{j}}_{e} \\ \text { [back] } & \sum \widehat{h}_{15}=\sum \widehat{h}_{12}-\sum \widehat{h}_{6}+\sum \widehat{h}_{7}-\sum \widehat{\hat{j}}_{d} .\end{aligned}$

One has to show now that with these values for $\widehat{h}_{i}$ the rotational equation (3.130b) is automatically satisfied on all remaining surfaces:

- Equations (A.16c) and (A.16d) for the left and right surface still hold true as before as they remain unchanged. 
- The rotational equation is fulfilled on the left parts of the front, upper, lower and back surfaces by construction. Thus, only the right parts of these surfaces still need to be considered. For symmetry reasons, it is sufficient to consider only one surface. Here the one in front is selected without loss of generality. The contour integral around this facet takes the form

$$
\begin{aligned}
& \sum \widehat{h}_{9}^{\prime}-\sum \widehat{h}_{5}-\sum \widehat{h}_{1}^{\prime}+\sum \widehat{h}_{14} \\
& \stackrel{\text { (A.17a) }}{=} \sum \widehat{h}_{9}^{\prime}-\sum \widehat{h}_{5}-\sum \widehat{h}_{1}^{\prime}+\sum \widehat{h}_{9}-\sum \widehat{h}_{1}+\sum \widehat{h}_{3}-\sum \widehat{j}_{b} \\
& \stackrel{\text { (A.16a) }}{=} \sum \widehat{\hat{j}}_{g} .
\end{aligned}
$$

Equation (3.130b) is thus satisfied on this surface.

- The last facet to be considered is the one in the middle, in the plane of the subdivision. The contour integral around this facet is given by

$$
\begin{aligned}
& \sum \widehat{h}_{15}-\sum \widehat{h}_{16}-\sum \widehat{h}_{14}+\sum \widehat{h}_{13} \\
& \stackrel{\text { (A.17) }}{=} \sum \widehat{h}_{7}-\sum \widehat{h}_{10}-\sum \widehat{h}_{3}+\sum \widehat{h}_{2}+\sum \widehat{\hat{j}}_{a}+\sum \widehat{\hat{j}}_{b}-\sum \widehat{\hat{j}}_{d}-\sum \widehat{\hat{j}}_{e} \\
& \stackrel{\text { (A.16c) }}{=} \sum \widehat{\hat{j}}_{a}+\sum \widehat{\hat{j}}_{b}+\sum \widehat{\hat{j}}_{c}-\sum \widehat{\hat{j}}_{d}-\sum \widehat{\hat{j}}_{e}=\sum \widehat{\hat{j}}_{k} \text {. }
\end{aligned}
$$

Hence, the rotational equation is fulfilled on the middle facet.

To sum up, it has been shown that equation (3.130b) is satisfied on all surfaces after each subdivision. 


\section{B Simplified class diagram of the combined magnetostatic and eigenvalue solver}

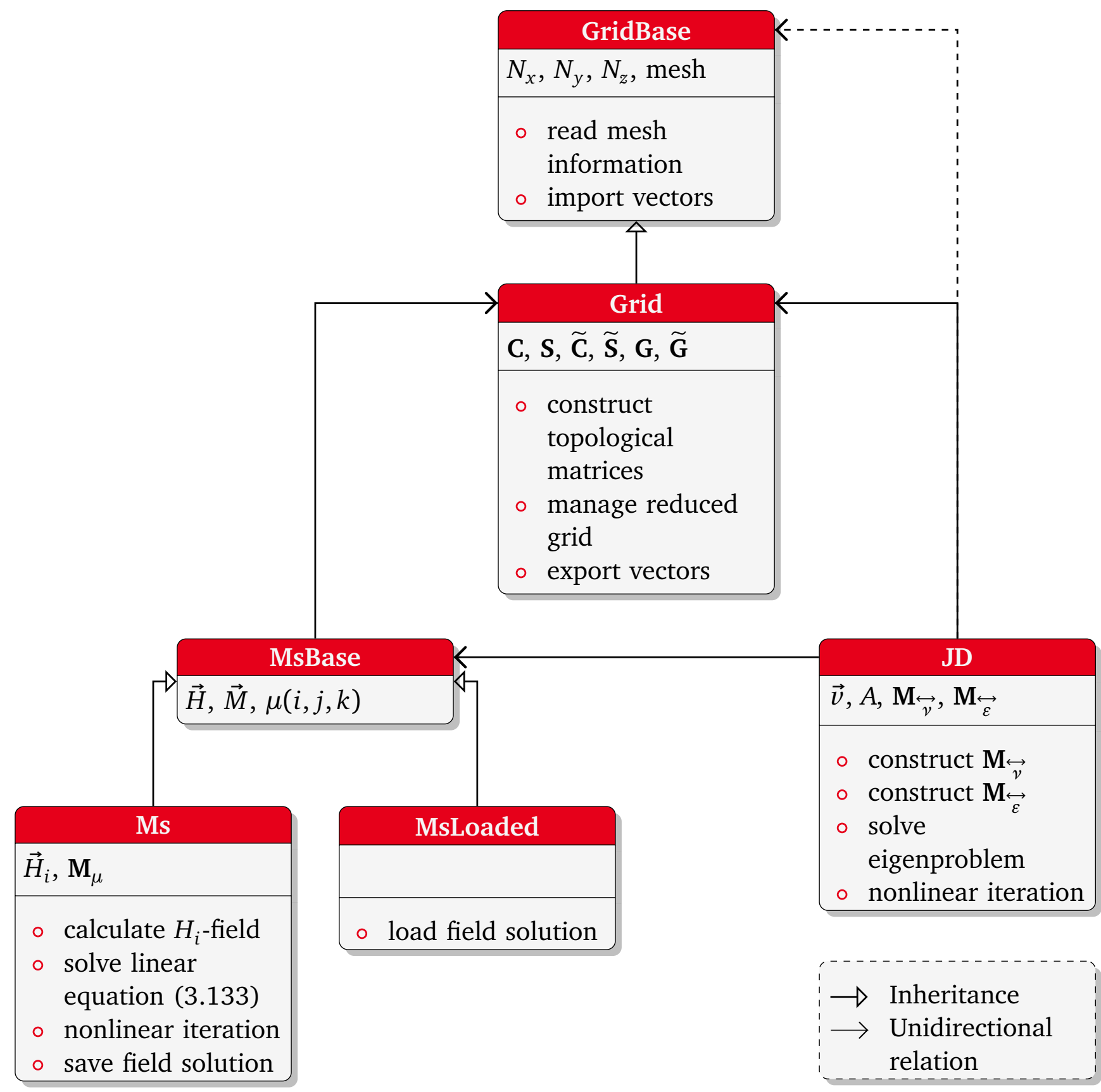

The cell-averaged values of the static magnetic field as well as the magnetization and the values of the permeability in each primary cell are saved after calculation by the $M s$-class before they are provided to the $J D$-class for the eigenmode computation. The saved magnetostatic solution can also be loaded by the MsLoaded-class. Moreover, the $J D$-class can optionally set imported field vectors as start vectors for the JD-iteration. 


\title{
List of abbreviations and symbols
}

\author{
Abbreviations \\ BLAS Basic Linear Algebra Subprograms \\ CAD Computer-aided design \\ CSR Compressed Sparse Row \\ DC Direct current \\ DOF Degrees of freedom \\ DUT Device under test \\ EH Mode which becomes a TM mode in the limit of vanishing $\kappa$ and $\varepsilon_{2}$ \\ FAIR Facility for Antiproton and Ion Research [3] \\ FIT Finite Integration Technique \\ GSI Institute for heavy-ion research (German: Gesellschaft für Schwerionenforschung) [1] \\ HE Mode which becomes a TE mode in the limit of vanishing $\kappa$ and $\varepsilon_{2}$ \\ JD Jacobi Davidson
}

LAPACK Linear Algebra Package [79]

MPI Message Passing Interface $[49,50]$

PEC Perfect electric conductor

PETSc Portable, Extensible Toolkit for Scientific Computation [46, 47]

RF Radio frequency

RQI Rayleigh quotient iteration

SIS Heavy-ion synchrotron (German: Schwerionensynchrotron)

TE Transversal electric

TM Transversal magnetic

VNA Vector network analyzer

General math

* Complex conjugate / conjugate transpose 
$1 \quad$ Unit vector / matrix

$\arg (a)$ Argument of $a$

$\partial \Omega \quad$ Domain boundary

det Determinant

$\vec{e}_{a} \quad$ Unit vector in direction $a$

i Imaginary unit

Im Imaginary part

$\vec{n} \quad$ Normal vector

$\nabla \quad$ Nabla operator

$\Phi \quad$ Azimuthal angle, cf. Fig. (2.4)

$\theta \quad$ Polar angle, cf. Fig. (2.4)

$\mathscr{O}$ Complexity class (computational complexity theory)

$\Omega \quad$ Domain

$\mathscr{P} \quad$ Transposition matrix

$A^{\perp} \quad$ Orthogonal projection of matrix $A$ on a subspace

$P_{\pi} \quad$ Permutation matrix

Re Real part

T Transpose

$J_{n} \quad$ Bessel function of the first kind of order $n$

$x_{n, m} \quad m$-th zero of $J_{n}$

\section{Eigensystems}

A System matrix

$\epsilon \quad$ Stopping criterion for eigenvalue iteration

$f_{\text {analy }}$ Analytical eigenfrequency

$k \quad$ Number of iterations (outer iteration)

$\mathscr{K} \quad$ Krylov subspace, cf. Eq. (3.155)

L $\quad$ Lower triangular matrix

U Upper triangular matrix

$\lambda \quad$ Eigenvalue 
M System matrix of projected system

$m \quad$ Number of iterations (inner iteration) / dimension of $\mathscr{V}$

$\omega_{\text {analy }}$ Analytical (circular) eigenfrequency

Q Orthogonal matrix

$\vec{r} \quad$ Residual vector

$R \quad$ Upper triangular matrix

$\vec{s} \quad$ Eigenvector of projected system

$\sigma \quad$ Shift / target value

$\vec{t} \quad$ Solution vector of JD correction equation

$T \quad$ Diagonal matrix

$\tau \quad$ Target value

$\theta \quad$ Approximation for eigenvalue / Ritz value

$\vec{u} \quad$ Approximation of eigenvector

$\vec{v} \quad$ Eigenvector

$V \quad$ Matrix of orthonormal columns which span subspace $\mathscr{V}$

$\mathscr{V} \quad$ Subspace spanned by columns of $V$

W Matrix of orthonormal columns which span subspace $\mathscr{W}$

$\mathscr{W} \quad$ Subspace spanned by columns of $W$

$\vec{y} \quad$ Ritz vector

\section{Finite Integration Technique}

A Classification of vector: $E$-field, $H$-field, normal node or dual node

A Primary facet / area of primary facet

$a_{\max } a$-coordinate $(a=x, y, z)$ at the border of the computation domain towards large indices

$a_{\text {min }} a$-coordinate $(a=x, y, z)$ at the border of the computation domain towards small indices

$\widetilde{A} \quad$ Dual facet / area of dual facet

$\widetilde{A}_{a}^{(n)}(i, j, k)$ Partial area of the the dual facet $\widetilde{A}_{a}(i, j, k)$ inside the normal cell $n$, cf. Fig. 3.3(b)
a $\quad$ Vector potential
$\widehat{\widehat{b}} \quad$ Magnetic flux
$\widehat{\mathrm{b}} \quad$ Vector collecting all magnetic fluxes 
C Discrete curl operator at the primary grid

$\mathscr{C}_{n} \quad$ Capacitance of a lumped element allocated on the primary edge / dual facet with index $n$

$\widetilde{\mathbf{C}} \quad$ Discrete curl operator at the dual grid

$\widehat{\widehat{d}} \quad$ Electric flux

$\widehat{\mathrm{d}} \quad$ Vector collecting all electric fluxes

$\mathbf{D}_{0}^{A} \quad$ Diagonal matrix for $A$-like vectors: $\left[\mathbf{D}_{0}^{A}\right]_{p, p}=1$ for ordinary components; $\left[\mathbf{D}_{0}^{A}\right]_{p, p}=0$ for elements associated with a pseudo-DOF

$\mathbf{D}_{A} \quad$ (Diagonal) vector collecting the areas of all primary facets

$\mathbf{D}_{\varepsilon} \quad$ (Diagonal) vector collecting the averaged values of the permittivity

$\mathbf{D}_{L} \quad$ (Diagonal) vector collecting the lengths of all primary edges

$\mathbf{D}_{v} \quad$ (Diagonal) vector collecting the averaged values of the inverse permeability

$\widetilde{\mathbf{D}}_{A} \quad$ (Diagonal) vector collecting the areas of all dual facets

$\widetilde{\mathbf{D}}_{L} \quad$ (Diagonal) vector collecting the lengths of all dual edges

$\Delta \quad$ Step size

$\delta \quad$ Threshold for magnetostatic solver

$\Delta_{a} \quad$ Change in the index when going from the $x$-component of one selected cell to the $a$ component $(a=x, y, z)$ of the same cell

$\widehat{e} \quad$ Electric voltage

e Vector collecting all electric voltages

$\bar{\varepsilon} \quad$ Average permittivity, cf. Eq. (3.29)

$E_{t} \quad$ Tangential component of the electric field

G Discrete gradient operator at the primary grid

$\widetilde{G} \quad$ Discrete gradient operator at the dual grid

$\widehat{\mathbf{h}} \quad$ Vector collecting all magnetic voltages

$\widehat{h} \quad$ Magnetic voltage

$\widehat{\mathbf{h}}_{\text {analy }}$ Magnetic voltages of the analytical solution

$\widehat{\mathbf{h}}_{h} \quad$ Irrotational magnetic field, cf. Eq. (3.130c)

$\widehat{\mathbf{h}}_{i} \quad$ Solenoidal magnetic field, cf. Eq. (3.130b)

$\bar{H}(n)$ Mean value for the magnetic field strength in the normal cell with index $n$

$\widehat{\mathbf{h}}_{\text {num }}$ Magnetic voltages of the numerical solution 
$\widehat{h}_{a}^{(i)}(n)$ Partial magnetic voltage that is allocated on the partial dual edge in the normal cell with index $i$, cf. Eq. (3.134)

$\vec{H}_{\text {res }} \quad$ Resulting magnetic field

$i \quad$ Cells number in direction $x$

j Cells number in direction $y$

$\widehat{\hat{j}} \quad$ (Electric) current

$\widehat{\mathbf{j}} \quad$ Vector collecting all (electric) currents

$\widehat{\hat{j}}_{C} \quad$ Current due to a lumped element capacitor

$\widehat{\hat{j}}_{R} \quad$ Current due to a lumped element resistor

$k \quad$ Cells number in direction $z$

$L \quad$ Primary edge / length of primary edge

$\widetilde{L} \quad$ Dual edge / length of dual edge

$\widetilde{L}_{a}^{(n)}(i, j, k)$ Length of the dual edge $\widetilde{L}_{a}(i, j, k)$ only of the part inside the normal cell $n$, cf. Fig. 3.2(b)

$\mathbf{M}_{\overline{0}} \quad$ Topological matrix without consideration of vanishing elements due to pseudo DOFs

$M_{a} \quad$ Number by which the position of a vector component / global cell index $n$ increases if one proceeds one cell in direction $a$

$[\mathbf{M}]_{(a, p),(b, q)}$ Element with row index $(a, p)$, i.e. index associated with vector component of cell with index $p$ in direction $a$, and column index $q$, i.e. index associated with vector component of cell with index $q$ in direction $b$, of matrix $\mathbf{M}$

$M_{a}^{\prime} \quad$ Number by which the position of a vector component $n$ increases if one proceeds one cell in direction $a$ for ordering scheme 1, cf. Eq. (3.37)

$M_{a}^{\prime \prime} \quad$ Number by which the position of a vector component $n$ increases if one proceeds one cell in direction $a$ for ordering scheme 2, cf. Eq. (3.50)

$\mathbf{M}_{C} \quad$ (Diagonal) lumped element capacitance matrix

$\mathbf{M}_{\varepsilon} \quad$ (Diagonal) permittivity matrix

$\mathbf{M}_{\stackrel{\varepsilon}{\leftrightarrow}} \quad$ Permittivity tensor

$\mathbf{M}_{v} \quad$ (Diagonal) inverse permeability matrix

$\mathbf{M}_{\overleftrightarrow{v}} \quad$ Inverse permeability tensor

$[\mathbf{M}]_{p, q}$ Element with row index $p$ and column index $q$ of matrix $\mathbf{M}$

$\mathbf{M}_{R} \quad$ (Diagonal) lumped element resistance matrix, cf. Eq. (3.123) 
$n \quad$ Position of a vector component / global cell index

$N_{a} \quad$ Number of cells in direction $a$

$N_{\mathrm{BC}}^{A} \quad$ Number of DOFs to be zeroed due to the imposed boundary conditions for A-like vectors

$N_{\text {cells }}$ Number of all (primary) grid cells

$N_{\text {DOF }}$ Number of DOFs (for the solution vector)

$N_{\text {DOF }}^{A}$ Number of DOFs for $A$-like vectors

$N_{\mathrm{NZ}} \quad$ Number of nonzeros

$N_{\text {outside }}^{A}$ Number of DOFs outside the computation domain for A-like vectors

$N_{\text {PEC }}^{A} \quad$ Number of DOFs to be zeroed due to elements inside PEC-cells or -wires for A-like vectors $N_{\text {pseudo-DOFs }}^{A}$ Number of pseudo-DOFs for $A$-like vectors

$N_{\text {removed }}$ Number of pseudo-DOFs that have already been removed before a particular element

$\bar{v} \quad$ Average inverse permeability, cf. Eq. (3.25)

p Row index (of a matrix)

$\mathbf{P}_{a} \quad$ Differential submatrix at the primary grid (discrete differential with respect to direction a) for ordering scheme 1, cf. Eq. (3.39)

${ }^{M} \mathbf{P}_{b}^{(a)}$ Differential matrix for topological matrices of type $M(M=\mathbf{C}, \S, \mathbf{G})$ at the primary grid for a general ordering scheme: Discrete differential with respect to direction $b$ acting on the $a$-components of the vector on which the operator is applied

$\Phi \quad$ Scalar potential, cf. Eq. (3.130c)

$\widetilde{\mathbf{P}}_{a} \quad$ Differential submatrix at the dual grid (discrete differential with respect to direction $a$ ) for ordering scheme 1, cf. Eq. (3.43)

${ }^{\mathrm{A}} \widetilde{\mathbf{P}}_{b}^{(a)}$ Differential matrix for topological matrices of type $A(A=\mathbf{C}, \S, \mathbf{G})$ at the dual grid for a general ordering scheme: Discrete differential with respect to direction $b$ acting on the $a$-components of the vector on which the operator is applied

$q \quad$ Column index (of a matrix)

$q \quad$ Electric charge

q Vector collecting all electric charges

$q_{\mathrm{m}} \quad$ Pseudo magnetic charge

$\mathbf{q}_{\mathrm{m}} \quad$ Vector collecting all pseudo magnetic charges

$R_{n} \quad$ Ohmic resistance of a lumped element allocated on the primary edge with index $n$

$\S \quad$ Discrete divergence operator at the primary grid 
$\widetilde{\mathbf{S}}$ Discrete divergence operator at the dual grid

$\sum \widehat{h}$ Sum of all $\widehat{\mathbf{h}}_{i}$-components along a (dual) edge

$\sum \widehat{\hat{j}}$ Sum of all currents through a (dual) surface

v Generic vector

V Primary volume

$\widetilde{V} \quad$ Dual volume

General constants and field quantities

Unit

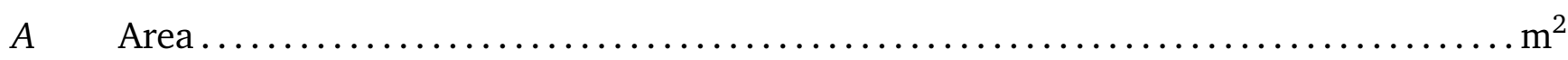

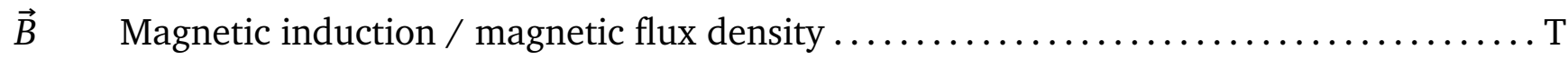

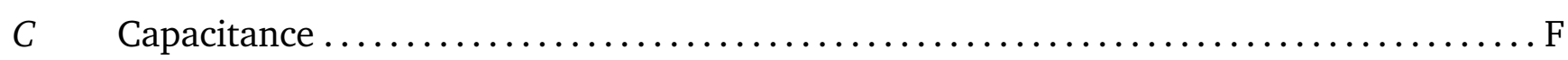

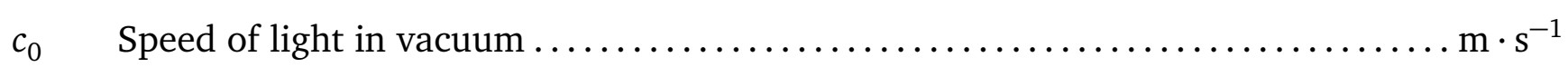

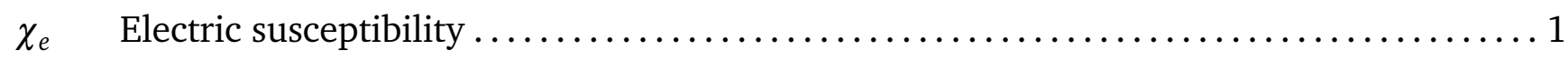

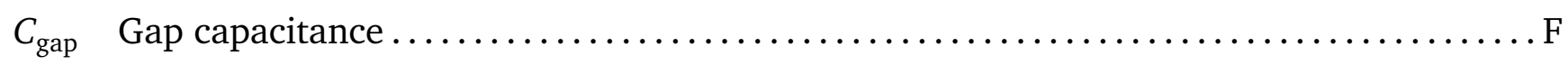

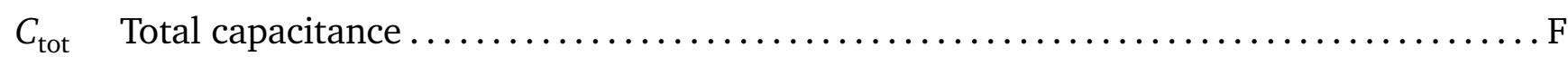

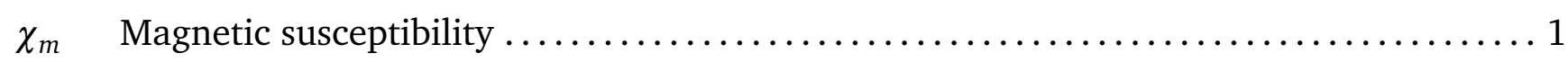

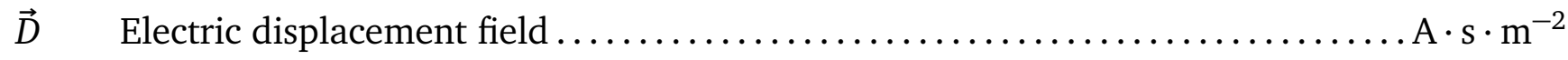

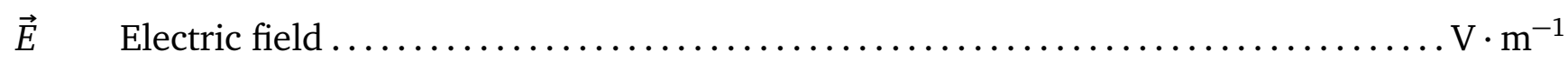

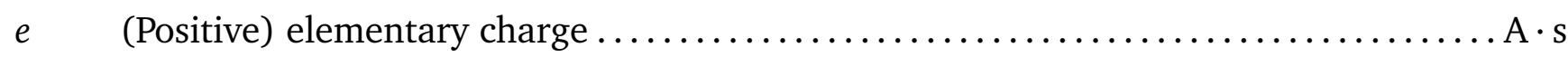

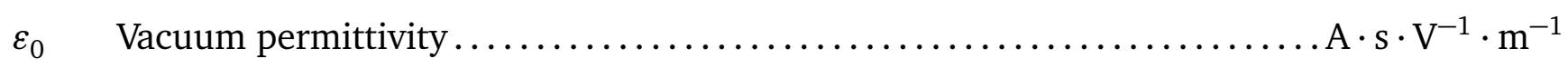

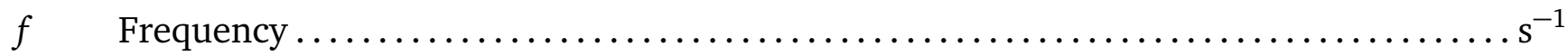

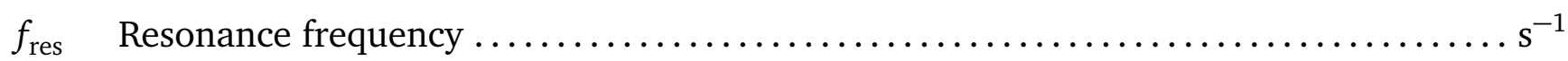

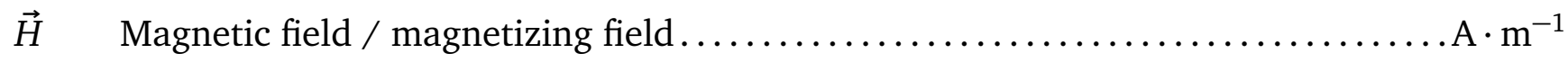

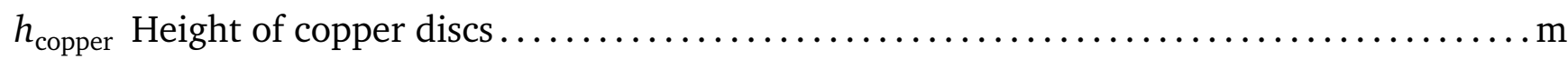

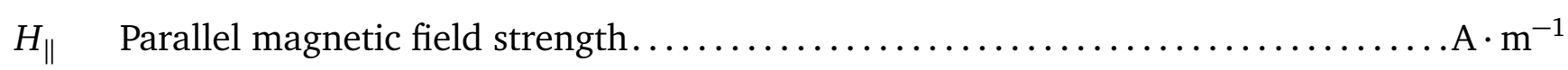

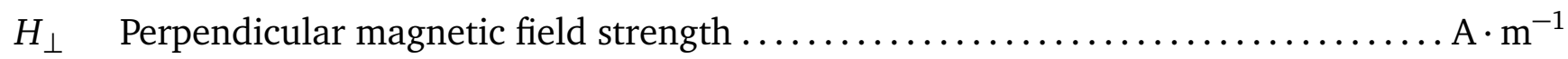

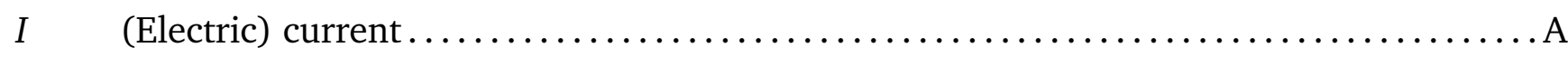

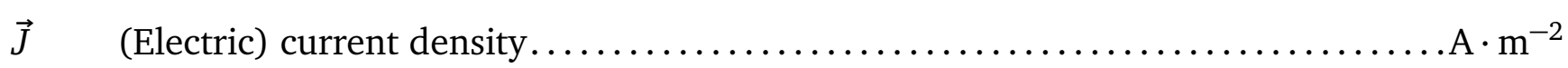

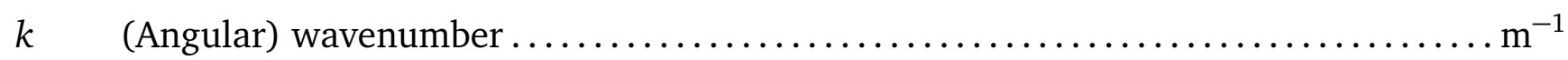

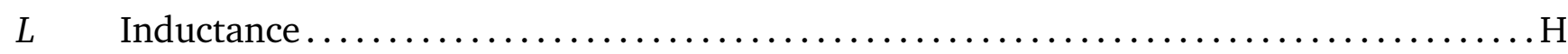


l Length. . $\mathrm{m}$

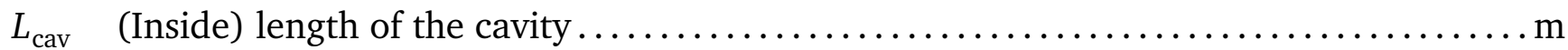

$\vec{M} \quad$ (Magnetic) polarization / magnetization field ...................... A $\mathrm{m}^{-1}$

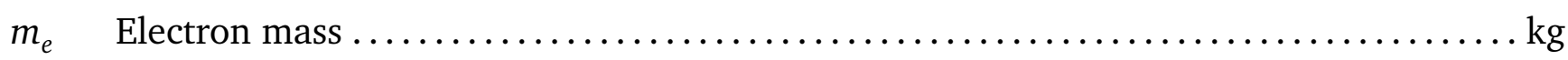

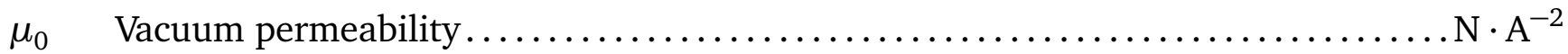

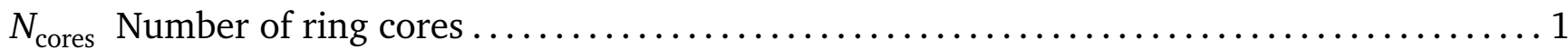

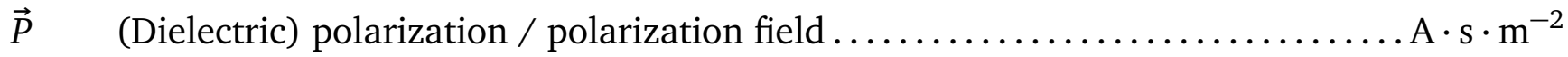

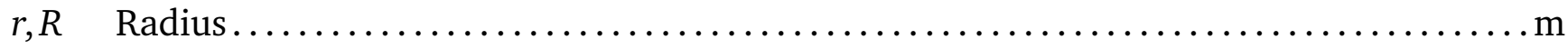

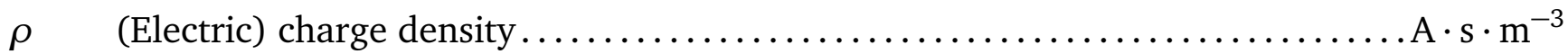

$\rho \quad$ Electric charge density $\ldots \ldots \ldots \ldots \ldots \ldots \ldots \ldots \ldots \ldots \ldots \ldots \ldots \ldots \ldots \ldots \ldots \ldots \ldots, \mathrm{m}^{-3}$

$\rho_{m} \quad$ Pseudo magnetic charge density $\ldots \ldots \ldots \ldots \ldots \ldots \ldots \ldots \ldots \ldots \ldots \ldots \ldots \ldots \ldots \ldots \ldots \ldots, \mathrm{m}^{-3}$

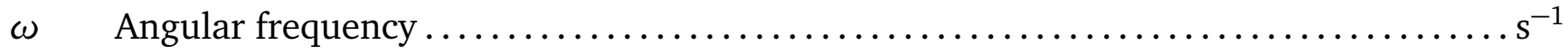

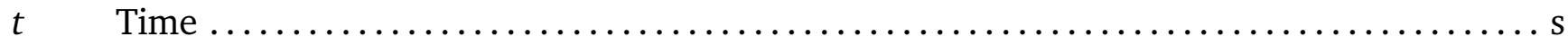

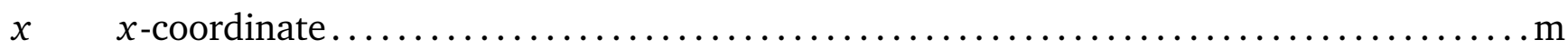

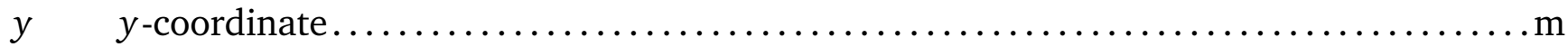

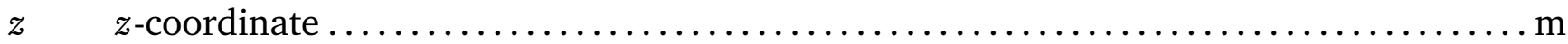

Material modeling and measurement Unit

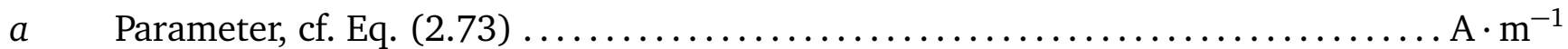

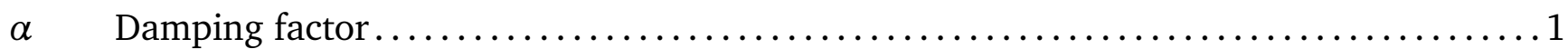

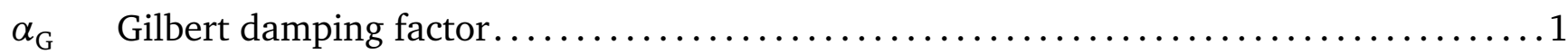

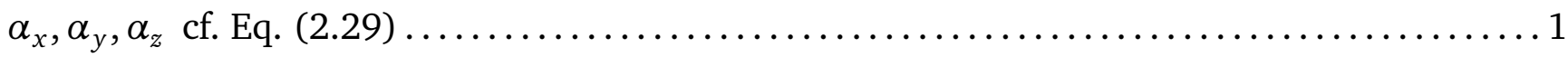

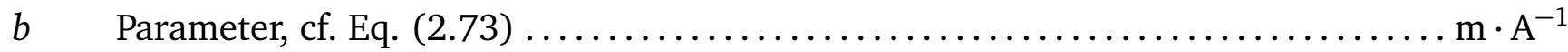

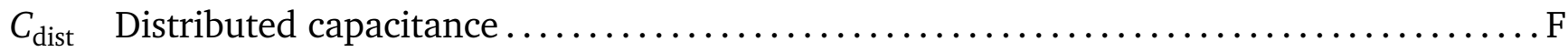

$C_{\text {ext }} \quad$ External capacitance..............................................

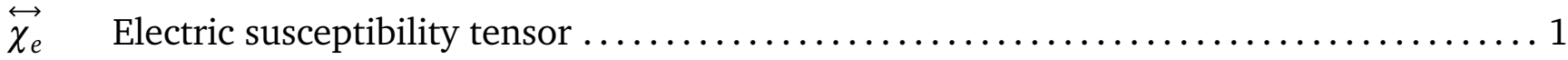

$\overleftrightarrow{\chi_{m}} \quad$ Magnetic susceptibility tensor.......................................

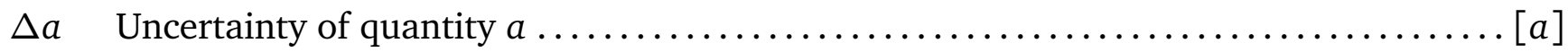

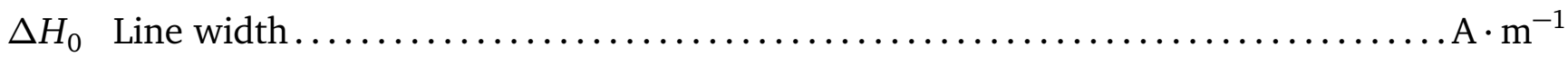

$\varepsilon_{1} \quad$ Main diagonal element of the permittivity tensor, cf. Eq. $(2.43) \ldots \ldots \ldots \ldots \ldots \ldots \ldots$ 
$\varepsilon_{2} \quad$ Off-diagonal element of the permittivity tensor, cf. Eq. $(2.43) \ldots \ldots \ldots \ldots \ldots \ldots \ldots$

$\varepsilon_{3} \quad Z$-component of the permittivity tensor, cf. Eq. $(2.43) \ldots \ldots \ldots \ldots \ldots \ldots \ldots \ldots \ldots$

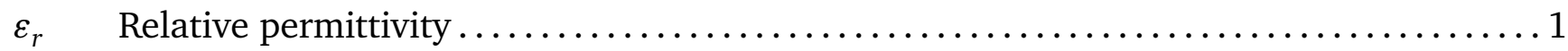

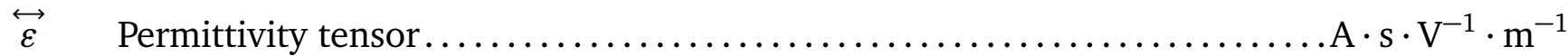

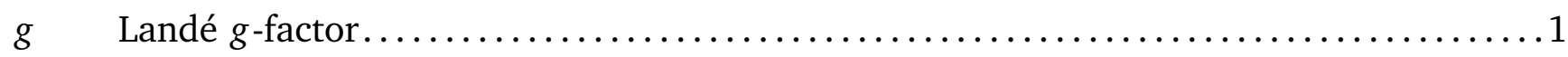

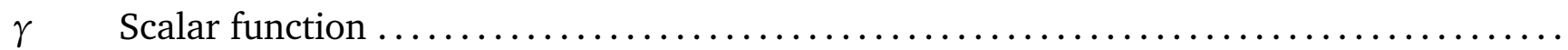

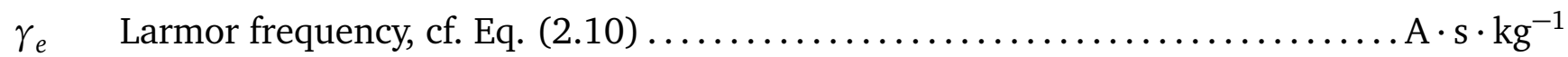

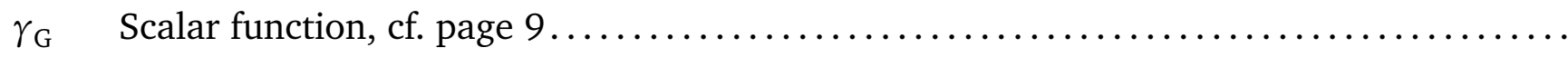

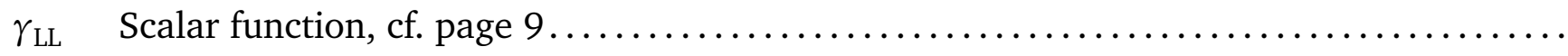

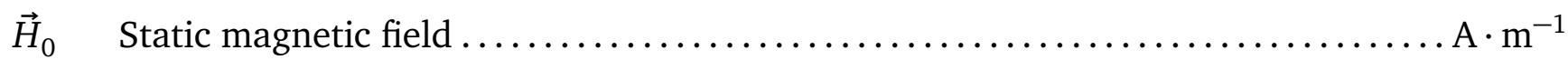

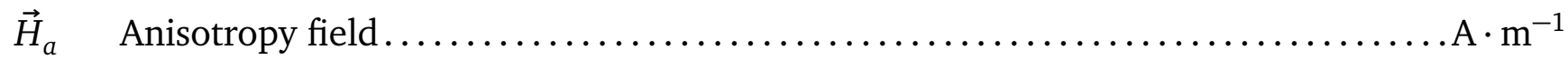

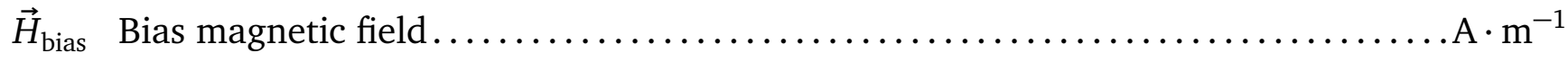

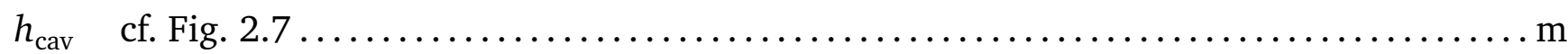

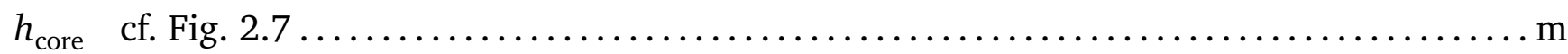

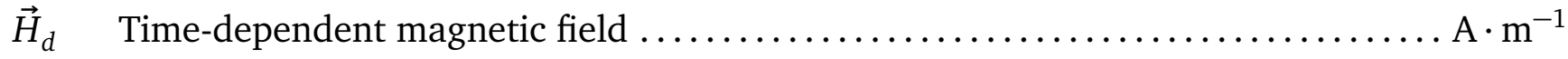

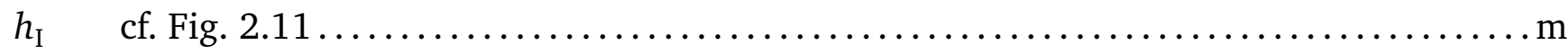

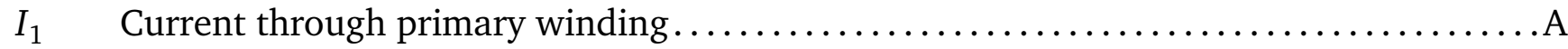

$I_{2} \quad$ Current through secondary winding $\ldots \ldots \ldots \ldots \ldots \ldots \ldots \ldots \ldots \ldots \ldots \ldots \ldots \ldots \ldots \ldots$

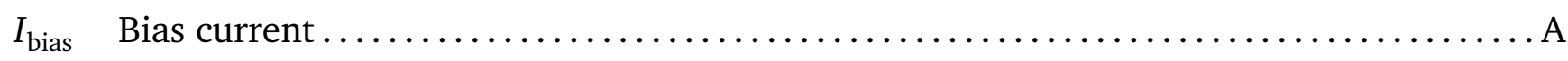

$\kappa \quad$ Off-diagonal element of permeability tensor (fully magnetized state), cf. Eq. (2.23) ... 1

$\widetilde{\kappa} \quad$ Off-diagonal element of permeability tensor (partially magnetized state) $\ldots \ldots \ldots \ldots 1$

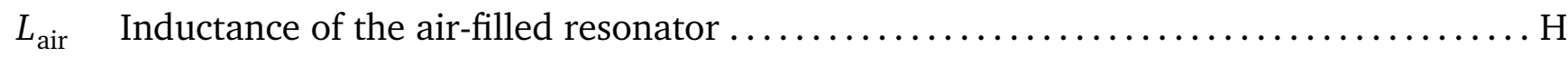

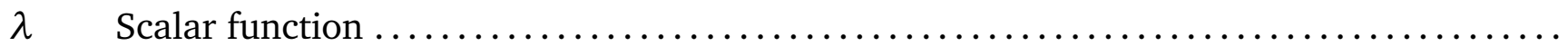

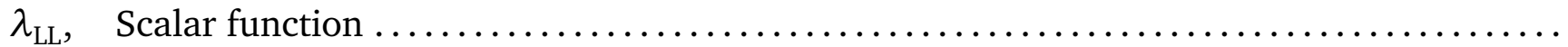

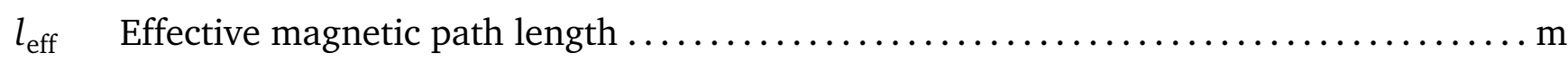

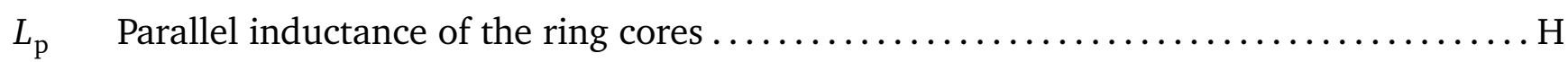

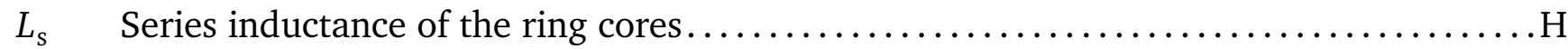

$m \quad$ Reduced magnetization $m=M / M_{\text {sat }}$ (partially magnetized state) $\ldots \ldots \ldots \ldots \ldots \ldots 1$

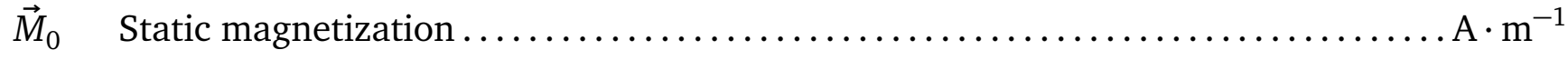


$\vec{M}_{d} \quad$ Time-dependent magnetization $A \cdot m^{-1}$

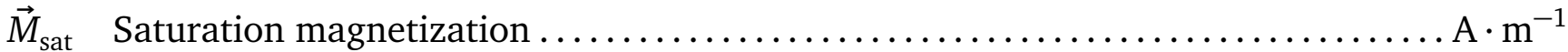

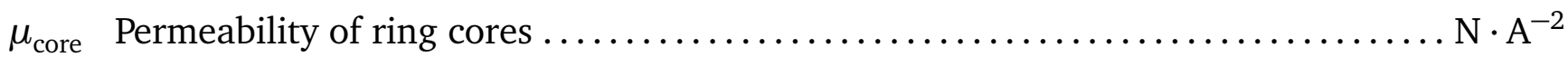

$\mu_{\text {diag }}$ Main diagonal element of permeability tensor (fully magnetized state), cf. Eq. (2.23) . . 1

$\tilde{\mu}_{\text {diag }}$ Main diagonal element of permeability tensor (partially magnetized state) ..........1

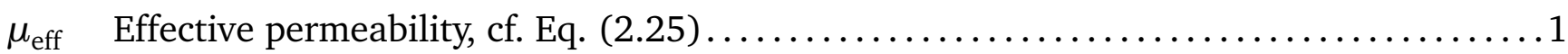

$\hat{\mu} \quad$ Permeability of the fully demagnetized state, cf. Eq. $(2.26) \ldots \ldots \ldots \ldots \ldots \ldots \ldots \ldots$

$\mu_{\mathrm{p}} \quad$ Permeability in the parallel equivalent circuit representation $\mu_{\mathrm{p}}^{-1}=\mu_{\mathrm{p}}^{\prime-1}+\mathrm{i} \mu_{\mathrm{p}}^{\prime \prime-1} \cdot \mathrm{N} \cdot \mathrm{A}^{-2}$

$\mu_{\mathrm{p}}^{\prime} \quad$ Real component of the permeability in the parallel equivalent circuit representation $\mathrm{N} \cdot \mathrm{A}^{-2}$

$\mu_{\mathrm{p}}^{\prime \prime} \quad$ Imaginary component of the permeability in the parallel equivalent circuit representation $\mathrm{N} \cdot \mathrm{A}^{-2}$

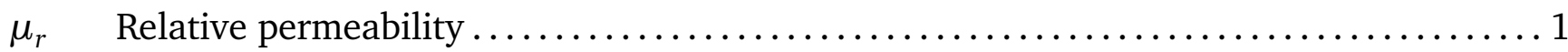

$\mu_{\mathrm{s}} \quad$ Permeability in the series equivalent circuit representation $\mu_{\mathrm{s}}=\mu_{\mathrm{s}}^{\prime}-\mathrm{i} \mu_{\mathrm{s}}^{\prime \prime} \ldots \ldots \mathrm{N} \cdot \mathrm{A}^{-2}$

$\mu_{\mathrm{s}}^{\prime} \quad$ Real component of the permeability in the series equivalent circuit representation $\mathrm{N} \cdot \mathrm{A}^{-2}$

$\mu_{\mathrm{s}}^{\prime \prime} \quad$ Imaginary component of the permeability in the series equivalent circuit representation $\mathrm{N} \cdot \mathrm{A}^{-2}$

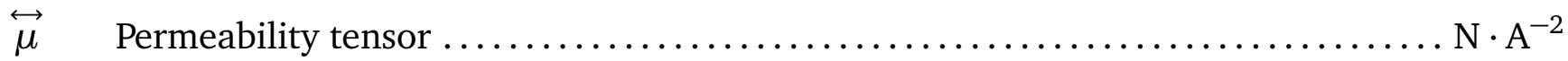

$\mu_{z} \quad Z$-component of permeability tensor (fully magnetized state), cf. Eq. (2.23).........1

$\tilde{\mu}_{z} \quad Z$-component of the permeability tensor (partially magnetized state) $\ldots \ldots \ldots \ldots \ldots 1$

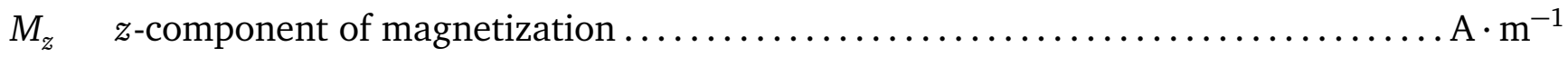

$N_{1} \quad$ Number of turns of primary winding $\ldots \ldots \ldots \ldots \ldots \ldots \ldots \ldots \ldots \ldots \ldots \ldots \ldots \ldots \ldots \ldots \ldots \ldots \ldots$

$N_{2} \quad$ Number of turns of secondary / sense winding $\ldots \ldots \ldots \ldots \ldots \ldots \ldots \ldots \ldots \ldots \ldots \ldots$

$N_{\text {bias }}$ Number of turns of bias current winding $\ldots \ldots \ldots \ldots \ldots \ldots \ldots \ldots \ldots \ldots \ldots \ldots \ldots \ldots \ldots \ldots \ldots$

$N_{e} \quad$ Number of electrons per unit volume $\ldots \ldots \ldots \ldots \ldots \ldots \ldots \ldots \ldots \ldots \ldots \ldots \ldots \ldots \ldots \ldots$

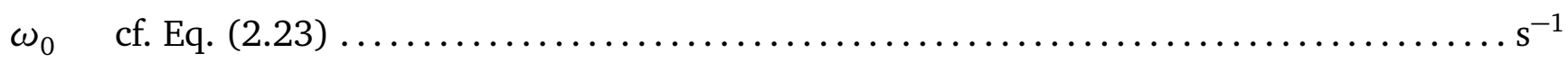

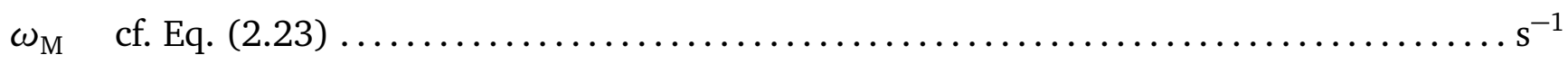

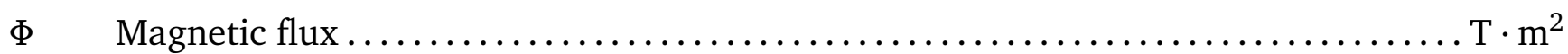

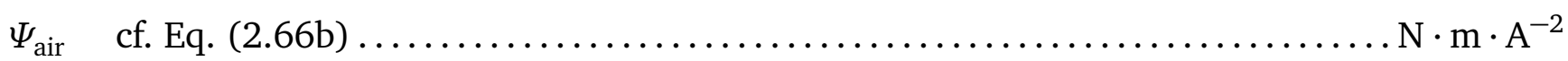

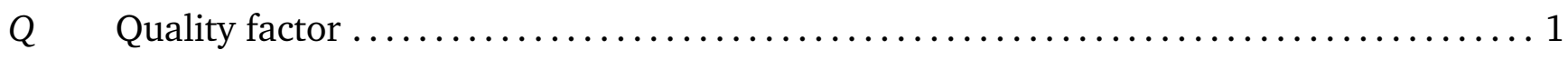




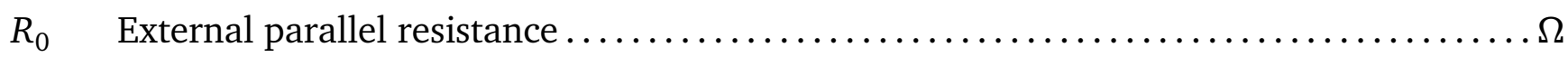

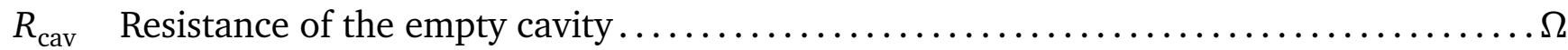

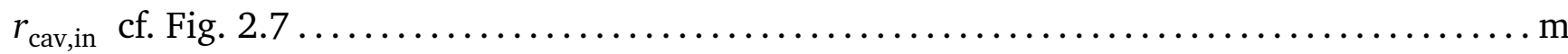

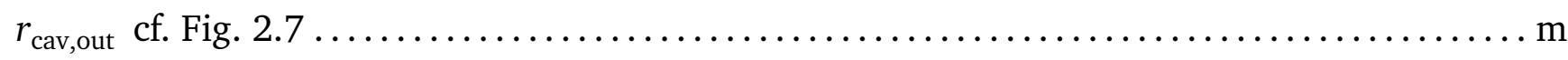

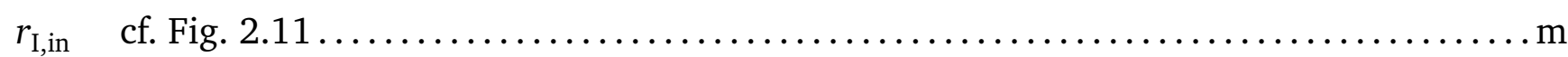

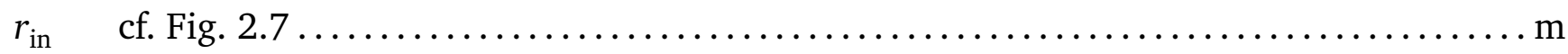

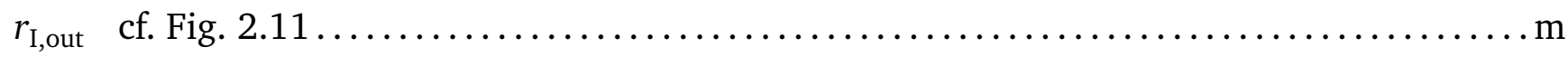

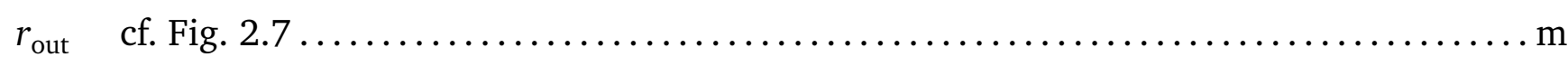

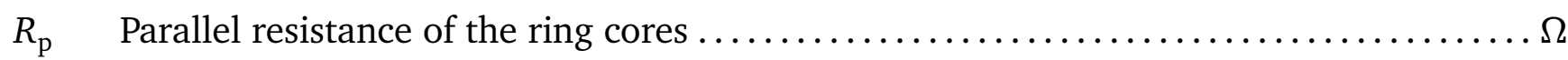

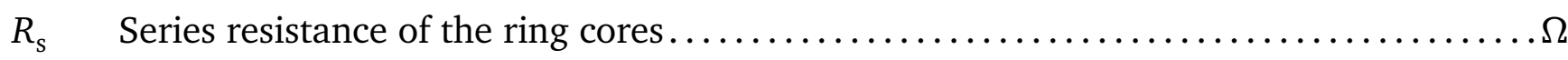

$S_{11} \quad$ Input port reflection coefficient $\ldots \ldots \ldots \ldots \ldots \ldots \ldots \ldots \ldots \ldots \ldots \ldots \ldots \ldots \ldots \ldots \ldots \ldots \ldots \ldots \ldots \ldots$

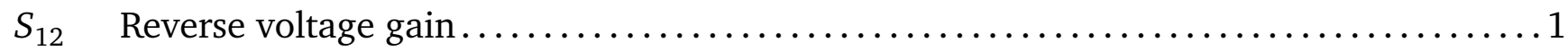

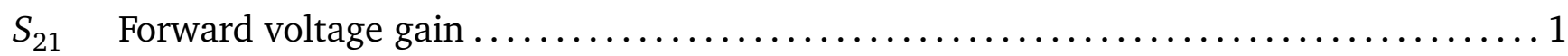

$S_{22} \quad$ Output port voltage reflection coefficient $\ldots \ldots \ldots \ldots \ldots \ldots \ldots \ldots \ldots \ldots \ldots \ldots \ldots \ldots$

$T_{1} \quad$ Relaxation time of the longitudinal magnetization, cf. Eq. $(2.15) \ldots \ldots \ldots \ldots \ldots \ldots$ s

$T_{2} \quad$ Relaxation time of the transversal magnetization, cf. Eq. $(2.15) \ldots \ldots \ldots \ldots \ldots$. . .

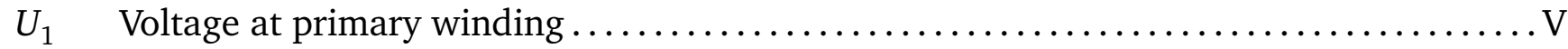

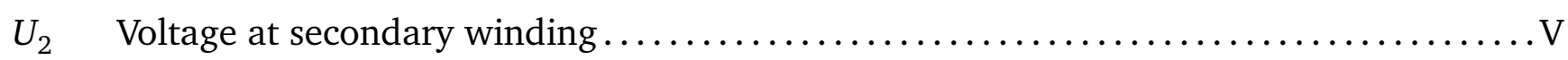

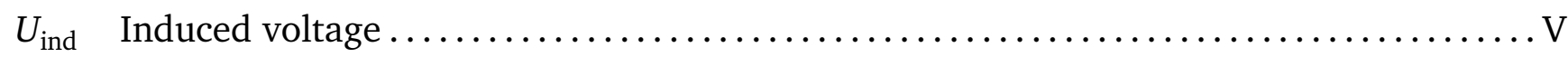

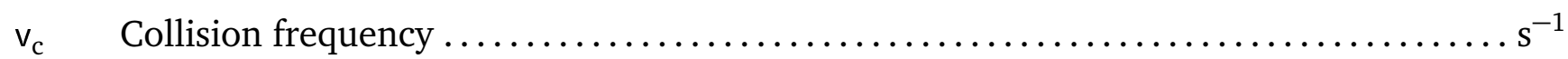

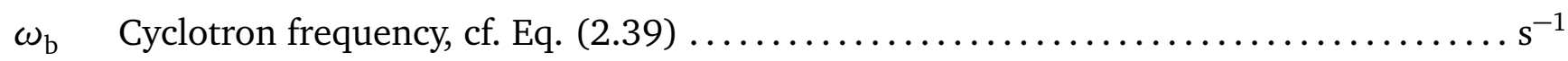

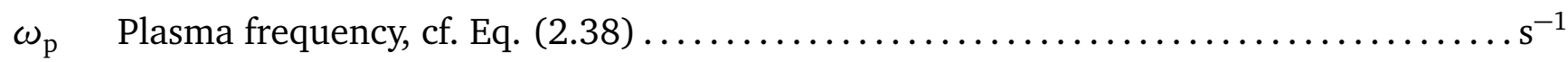

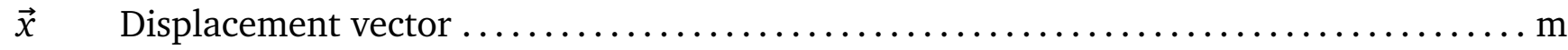

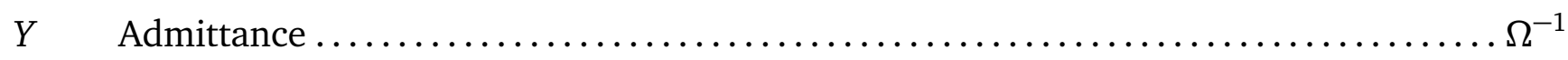

$Y_{0} \quad$ Admittance without DUT, cf. Eq. $(2.47) \ldots \ldots \ldots \ldots \ldots \ldots \ldots \ldots \ldots \ldots \ldots \ldots \ldots \ldots \ldots$

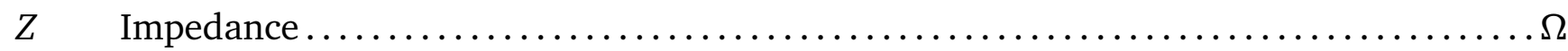

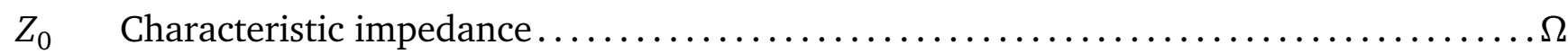

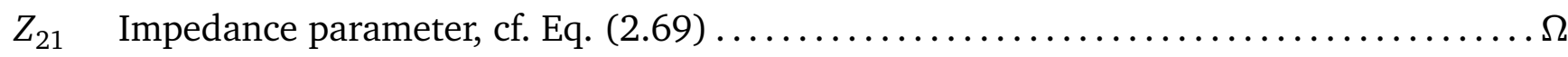




\section{Bibliography}

[1] GSI Helmholtzzentrum für Schwerionenforschung GmbH, homepage. Web page: https: //www.gsi.de/.

[2] H. Klingbeil. Ferrite cavities. CERN Yellow Report CERN-2011-007, pp. 299-317, 2012. arXiv: 1201.1154 .

[3] FAIR - Facility for Antiproton and Ion Research in Europe GmbH, homepage. Web page: http://www . fair-center. de.

[4] John David Jackson. Classical Electrodynamics. John Wiley \& Sons, New Jersey, 3rd edition, 1999.

[5] Dieter Meschede. Gerthsen Physik. Springer, Berlin, 23rd edition, 2006.

[6] Ronald F. Soohoo. Theory and application of ferrites. Prentice Hall, Englewood Cliffs, 1960.

[7] R.P. Feynmann, R.B. Leighton, and M. Sands. The Feynmann Lecture on Physics, volume I. Addison-Wesley Publishing Company, Reading, 1977.

[8] Herbert B. Callen. A ferromagnetic dynamical equation. J. Phys. Chem. Solids, 4(4):256 - 270, 1958. doi : 10.1016/0022-3697(58)90077-5.

[9] L. D. Landau and E. M. Lifshitz. Theory of the dispersion of magnetic permeability in ferromagnetic bodies. Phys. Z. Sowjetunion, 8(135), 1935.

[10] W. M. Saslow. Landau-Lifshitz or Gilbert damping? That is the question. J. Appl. Phys., 105(7), 2009. doi : 10.1063/1.3077204.

[11] T.L. Gilbert. A phenomenological theory of damping in ferromagnetic materials. IEEE Trans. Magn., 40(6):3443-3449, Nov 2004. doi : 10.1109/TMAG. 2004.836740.

[12] Janis Kliava. Electron Magnetic Resonance of Nanoparticles: Superparamagnetic Resonance, pages 255-302. Wiley, Weinheim, 2009. doi : 10.1002/9783527627561.ch7.

[13] F. Bloch. Nuclear Induction. Phys. Rev., 70:460-474, Oct 1946. doi : 10.1103/PhysRev. 70.460 .

[14] N. Bloembergen. On the Ferromagnetic Resonance in Nickel and Supermalloy. Phys. Rev., 78:572-580, June 1950. doi : 10.1103/PhysRev . 78 . 572.

[15] B. Kaviraj and S. K. Ghatak. Stress - and Magneto-Impedance in Co71-xFexCr7Si8B14 (x $=0,2)$ amorphous ribbons, 2006. arXiv: cond-mat/0604438.

[16] D. Polder. On the theory of ferromagnetic resonance. Philos. Mag. 7, 40(300):99-115, 1949. doi : $10.1080 / 14786444908561215$. 
[17] D. Bariou, Patrick Queffelec, Philippe Gelin, and M. Le Floc'h. Extension of the effective medium approximation for determination of the permeability tensor of unsaturated polycrystalline ferrites. IEEE Trans. Magn., 37(6):3885-3891, Nov 2001. doi: 10.1109/20.966123.

[18] Ernst Schlömann. Microwave Behavior of Partially Magnetized Ferrites. J. Appl. Phys., 41(1):204-214, 1970. doi : 10.1063/1.1658322.

[19] M. Igarashi and Yoshiyuki Naito. Tensor permeability of partially magnetized ferrites. IEEE Trans. Magn., 13(5):1664-1668, Sep 1977. doi : 10.1109/TMAG. 1977. 1059669.

[20] Philippe Gelin and Patrick Queffelec. Generalized Permeability Tensor Model: Application to Barium Hexaferrite in a Remanent State for Self-Biased Circulators. IEEE Trans. Magn., 44(1):24-31, Jan 2008. doi : 10.1109/TMAG. 2007. 909561.

[21] J. P. Bouchaud and P. G. Zérah. Spontaneous resonances and universal behavior in ferrimagnets: Effective-medium theory. Phys. Rev. Lett., 63:1000-1003, Aug 1989. doi : $10.1103 /$ PhysRevLett . 63.1000 .

[22] George T. Rado. Theory of the Microwave Permeability Tensor and Faraday Effect in Nonsaturated Ferromagnetic Materials. Phys. Rev., 89:529-529, Jan 1953. doi : 10.1103/ PhysRev. 89.529.

[23] Jerome J. Green and Frank Sandy. Microwave Characterization of Partially Magnetized Ferrites. IEEE Trans. Microw. Theory Techn., 22(6):641-645, Jun 1974. doi:10.1109/ TMTT . 1974.1128306.

[24] M. Igarashi and Y. Naito. Parallel Component mu_z of Partially Magnetized Microwave Ferrites. IEEE Trans. Microw. Theory Techn., 29(6):568-571, Jun 1981. doi:10.1109/ TMTT . 1981.1130394.

[25] P. Gelin. New consistent model for ferrite permeability tensor with arbitrary magnetization state. IEEE Trans. Microw. Theory Techn., 45(8):1185-1192, Aug 1997. doi : $10.1109 / 22.618406$.

[26] D. Polder and J. Smit. Resonance Phenomena in Ferrites. Rev. Mod. Phys., 25:89-90, Jan 1953. doi : 10.1103/RevModPhys . 25.89.

[27] E. C. Stoner and E. P. Wohlfarth. A mechanism of magnetic hysteresis in heterogeneous alloys. IEEE Trans. Magn., 27(4):3475-3518, Jul 1991. doi : 10. 1109/TMAG. 1991 . 1183750.

[28] G. Tyras. The Permeability Matrix for a Ferrite Medium Magnetized at an Arbitrary Direction and its Eigenvalues (Correspondence). IRE Trans. Microw. Theory Tech., 7(1):176177, January 1959. doi : 10.1109/TMTT . 1959. 1124645.

[29] V. L. Ginzburg. The propagation of electromagnetic waves in plasmas. Pergammon Press, Oxford, 1964.

[30] Klaus Klopfer, Uwe Niedermayer, Harald Klingbeil, Wolfgang Ackermann, Hans Günther König, and Thomas Weiland. Measurement of the magnetic material properties and simulation and modeling of ferrite-loaded cavities. submitted for publication, 2014. 
[31] Delta Elektronika Data sheet SM3000-series, 2012.

[32] R\&S ZNB Vector Network Analyzers User Manual, 2012.

[33] Fluke 62 Mini Infrared Thermometer Manual. Web page: http://www.fluke.com/ fluke/usen/electrical-test-tools/thermometers/fluke-62.htm?PID=56096.

[34] Qin Yu, T.W. Holmes, and K. Naishadham. RF equivalent circuit modeling of ferritecore inductors and characterization of core materials. IEEE Trans. Electromagn. Compat., 44(1):258-262, Feb 2002. doi : 10.1109/15 . 990733.

[35] Agilent Solutions for Measuring Permittivity and Permeability with LCR Meters and Impedance Analyzers, 2008. Application Note 1369-1.

[36] Jing Shenhui and Jiang Quanxing. An alternative method to determine the initial permeability of ferrite core using network analyzer. IEEE Trans. Electromagn. Compat., 47(3):651-657, Aug 2005. doi : 10.1109/TEMC. 2005.853169.

[37] Accurate Measurement of Ferrite Garnets to be used for Fast-tuned Ferrite Loaded Cavities in the Range of 20-40 MHz, Proceedings, 3rd International Particle Accelerator Conference (IPAC 2012), 2012. THPPC020.

[38] V.J. Thottuvelil, T.G. Wilson, and Jr. Owen, H.A. High-frequency measurement techniques for magnetic cores. IEEE Trans. Power Electron., 5(1):41-53, Jan 1990. doi:10.1109/ 63.45998.

[39] C. Vollinger, F. Caspers, and E. Jensen. The Effect of 2-Directional Magnetic Biasing Used for Tuning of a Ferrite-Loaded Re-entrant Cavity. IEEE Trans. Nucl. Sci., 60(3):2170-2174, June 2013. doi : 10.1109/TNS. 2013.2256148.

[40] David M. Pozar. Microwave Engineering. John Wiley \& Sons, New Jersey, 3rd edition, 2005.

[41] Soft Ferrites and Accessories, 2009.

[42] TDK EMC TECHNOLOGY-GUIDE BOOK FOR EMC, 2011. Web page: http://www.tdk. co.jp/emc_guide_e/.

[43] R\&S ZNB Vector Network Analyzers Specifications, 2013. version 06.00.

[44] F. G. Brockman, H. van der Heide, and M. W. Louwerse. Ferroxcube für Protonensynchrotrons. Philips Technische Rundschau, 1969/70.

[45] Anders Bergqvist. Magnetic vector hysteresis model with dry friction-like pinning. Physica B: Condensed Matter, 233(4):342 - 347, 1997. doi : 10 .1016/S0921-4526(97)00319-0.

[46] Satish Balay, Jed Brown, Kris Buschelman, William D. Gropp, Dinesh Kaushik, Matthew G. Knepley, Lois Curfman McInnes, Barry F. Smith, and Hong Zhang. PETSc Web page, 2012. Web page: http://www.mcs.anl.gov/petsc. 
[47] Satish Balay, Shrirang Abhyankar, Mark F. Adams, Jed Brown, Peter Brune, Kris Buschelman, Victor Eijkhout, William D. Gropp, Dinesh Kaushik, Matthew G. Knepley, Lois Curfman McInnes, Karl Rupp, Barry F. Smith, and Hong Zhang. PETSc users manual. Technical Report ANL-95/11 - Revision 3.4, Argonne National Laboratory, 2012. Web page: http://www.mcs.anl.gov/petsc.

[48] CST STUDIO SUITE®. CST Computer Simulation Technology AG, Bad Nauheimer Str. 19, Darmstadt, Germany. Web page: https://www.cst.com.

[49] The MPI Forum. MPI: A Message Passing Interface, 1993.

[50] MPI: A message-passing interface standard. Int J Supercomput Appl High Perform Comput, 8(3/4):159-416, 1994.

[51] Yousef Saad. Iterative Methods for Sparse Linear Systems. Society for Industrial and Applied Mathematics, 2nd edition, 2003. Web page: http://www-users.cs.umn.edu/ $\sim$ saad/books.html.

[52] Thomas Weiland. Verfahren und Anwendungen der Feldsimulation I, 2012. lecture notes.

[53] Thomas Weiland. Eine Methode zur Lösung der Maxwellschen Gleichungen für sechskomponentige Felder auf diskreter Basis. Electronics and Communications (AË̈), 31(3):116-120, 31977.

[54] Paul E. Black. binary search. Dictionary of Algorithms and Data Structures, 2013. National Institute of Standards and Technology (NIST). Web page: http://xlinux.nist. gov/dads//HTML/binarySearch.html.

[55] Holger Spachmann, Stefan Gutschling, Holm Krüger, and Thomas Weiland. FITformulation for non-linear dispersive media. Int. J. Numer. Model., 12(1-2):81-92, 1999. doi : 10 . 1002/(SICI) 1099-1204(199901/04) 12:1/2<81: :AID- JNM324>3 . 0 . C0;2-L.

[56] S. Gutschling, H. Krüger, and T. Weiland. Time-domain simulation of dispersive media with the finite integration technique. Int. J. Numer. Model., 13(4):329-348, 2000. doi : 10. 1002/1099-1204(200007/08) 13:4<329: : AID- JNM383>3 . 0. C0;2-C.

[57] Holm Krüger. Zur numerischen Berechnung transienter elektromagnetischer Felder in gyrotropen Materialien. PhD thesis, Darmstadt Univ. of Technol., 2000.

[58] S. Feigh, M. Clemens, R. Schuhmann, and T. Weiland. Eigenmode simulation of electromagnetic resonator cavities with gyrotropic materials. IEEE Trans. Magn., 40(2):647-650, 2004.

[59] Klaus Klopfer, Wolfgang Ackermann, and Thomas Weiland. Computation of Complex Eigenmodes for Resonators Filled With Gyrotropic Materials. accepted for publication, 2014. doi : 10.1109/TMAG . 2014.2338275.

[60] R. Holland. Finite-Difference Solution of Maxwell's Equations in Generalized Nonorthogonal Coordinates. IEEE Trans. Nucl. Sci., 30(6):4589-4591, Dec 1983. doi:10.1109/ TNS. 1983.4333176. 
[61] R. Schuhmann and T. Weiland. A stable interpolation technique for FDTD on nonorthogonal grids. Int. J. Numer. Model., 11(6):299-306, 1998. doi:10.1002/(SICI) 1099-1204 (199811/12) 11:6<299: :AID- JNM314>3 . 0 . CO;2-A.

[62] Wenquan Sui, Douglas A. Christensen, and C.H. Durney. Extending the two-dimensional FDTD method to hybrid electromagnetic systems with active and passive lumped elements. IRE Trans. Microw. Theory Tech., 40(4):724-730, Apr 1992. doi:10.1109/22. 127522 .

[63] Jason Mix, Jonathan Dixon, Zoya Popovic, and Melinda Piket-May. Incorporating nonlinear lumped elements in FDTD: the equivalent source method. Int. J. Numer. Model., 12(1-2):157-170, 1999. doi:10.1002/(SICI) 1099-1204(199901/04) 12:1/2<157: : AID- JNM323>3.0.CO; $2-\mathrm{V}$.

[64] Uta Hartel. Modellierung des Regelungs- und Steuerungssystems einer Beschleunigungseinheit für Synchrotrons. Diploma thesis, Darmstadt Univ. of Technol., 2011.

[65] George B. Arfken, Hans J. Weber, and Frank E. Harris. Mathematical Methods for Physicists. Academic Press, San Diego, 4th edition, 1995.

[66] E. Dlala and A. Arkkio. Analysis of the Convergence of the Fixed-Point Method Used for Solving Nonlinear Rotational Magnetic Field Problems. IEEE Trans. Magn., 44(4):473478, April 2008. doi : 10.1109/TMAG.2007.914888.

[67] Martin Schauer. Ein Beitrag zur Simulation statischer und niederfrequenter Felder in nichtlinearen und hochpermeablen Materialien. PhD thesis, Darmstadt Univ. of Technol., 2005.

[68] H. De Gersem, I. Munteanu, and T. Weiland. Construction of Differential Material Matrices for the Orthogonal Finite-Integration Technique With Nonlinear Materials. IEEE Trans. Magn., 44(6):710-713, June 2008. doi : 10. 1109/TMAG . 2007 . 915819.

[69] Herbert De Gersem, 2011. private communication.

[70] Bernhard Wagner. Numerische Simulation von elektromagnetischen Feldern in nichtlinearen Materialien. PhD thesis, Darmstadt Univ. of Technol., 1996.

[71] N.H. Abel. Beweis der Unmöglichkeit, algebraische Gleichungen von höheren Graden als dem vierten allgemein aufzulösen. J. Reine Angew. Math., 1:65-84, 1826. Web page: http: //eudml .org/doc/183015.

[72] Yousef Saad. Numerical Methods for Large Eigenvalue Problems. Society for Industrial and Applied Mathematics, 2nd edition, 2011. Web page: http://www-users.cs.umn.edu/ $\sim$ saad/books . html.

[73] Beresford N. Parlett. The Symmetric Eigenvalue Problem. Society for Industrial and Applied Mathematics, Philadelphia, 1997.

[74] Z. Bai, J. Demmel, J. Dongarra, A. Ruhe, and H. van der Vorst, editors. Templates for the Solution of Algebraic Eigenvalue Problems: A Practical Guide. Society for Industrial and Applied Mathematics, Philadelphia, 2000. Web page: http://web.eecs.utk.edu/ dongarra/etemplates/book . html. 
[75] Gene H. Golub and Charles F. van Van Loan. Matrix Computations. Johns Hopkins University Press, 3rd edition, 1996.

[76] I. N. Bronstein and K. A. Semendjajew. Taschenbuch der Mathematik. Harri Deutsch, Thun, 6th edition, 2006.

[77] R. Granat, B. Kågström, and D. Kressner. Parallel eigenvalue reordering in real Schur forms. CONCURR COMP-PRACT E, 21(9):1225-1250, 2009. doi : 10. 1002/cpe . 1386.

[78] HansRudolf Schwarz and Norbert Köckler. Eigenwertprobleme. In Numerische Mathematik, pages 218-273. Vieweg+Teubner Verlag, Wiesbaden, 2011. doi:10.1007/ 978-3-8348-8166-3_6.

[79] E. Anderson, Z. Bai, C. Bischof, S. Blackford, J. Demmel, J. Dongarra, J. Du Croz, A. Greenbaum, S. Hammarling, A. McKenney, and D. Sorensen. LAPACK Users' Guide. Society for Industrial and Applied Mathematics, Philadelphia, 3rd edition, 1999. Web page: http://www . netlib.org/lapack/lug/.

[80] Walter Ritz. Über eine neue Methode zur Lösung gewisser Variationsprobleme der mathematischen Physik. J. Reine Angew. Math., 135(1):1-61, 1909. Web page: http: //gdz.sub. uni-goettingen . de/no_cache/dms/load/img/?IDDOC=261182.

[81] Zhongxiao Jia and G. W. Stewart. On the Convergence of Ritz Values, Ritz Vectors, and Refined Ritz Vectors. Technical report, Institute for Advanced Computer Studies, University of Maryland, 1999.

[82] Michiel E. Hochstenbach. Generalizations of harmonic and refined rayleigh-ritz. Electron. Trans. Numer. Anal., 20:235-252, 2005. Web page: http://eudml.org/doc/128022.

[83] Chris C. Paige, Beresford N. Parlett, and Henk A. van der Vorst. Approximate solutions and eigenvalue bounds from Krylov subspaces. Numer. Linear Algebra Appl., 2(2):115133, 1995. doi : 10.1002/nla. 1680020205.

[84] Zhongxiao Jia. The convergence of harmonic Ritz values, harmonic Ritz vectors, and refined harmonic Ritz vectors. Math. Comp., 74(251):1441-1456 (electronic), 2005. doi : $10.1090 /$ S0025-5718-04-01684-9.

[85] Zhongxiao Jia. Über ein leichtes Verfahren die in der Theorie der Säcularstörungen vorkommenden Gleichungen numerisch aufzulösen. J. Reine Angew. Math., 1846(30):51-94, Jan 1846. doi : 10.1515/crll .1846.30.51.

[86] Jane Cullum. Arnoldi versus nonsymmetric lanczos algorithms for solving matrix eigenvalue problems. BIT Numer. Math., 36(3):470-493, 1996. doi : 10.1007/BF01731928.

[87] Gerard L. G. Sleijpen and Henk A. Van der Vorst. A Jacobi-Davidson Iteration Method for Linear Eigenvalue Problems. SIAM Rev., 42(2):267-293, June 2000. doi : 10.1137/ S0036144599363084.

[88] Eric de Sturler. Improving the Convergence of the Jacobi-Davidson Algorithm. Technical report, University of Illinois at Urbana-Champaign, 2000. UIUCDCS-R2000-2173. Web page: http://www.cse.uiuc.edu/ sturler/Public/tech-reports/ UIUCDCS-R-2000-2173.ps. 
[89] D. Fokkema, G. Sleijpen, and H. Van der Vorst. Jacobi-Davidson Style QR and QZ Algorithms for the Reduction of Matrix Pencils. SIAM J. Sci. Comput., 20(1):94-125, 1998. doi:10.1137/S1064827596300073.

[90] M.E. Hochstenbach and Y. Notay. The Jacobi-Davidson method. GAMM-Mitteilungen, 29(2):368-382, 2006. doi : 10.1002/gamm. 201490038.

[91] Gerard L.G. Sleijpen, Henk A. van der Horst, and Ellen Meijerbrink. Efficient expansion of subspaces in the Jacobi-Davidson method for standard and generalized eigenproblems. Electron. Trans. Numer. Anal., 7:75-89, 1998. Web page: http://eudml.org/doc/ 119748.

[92] D. Fokkema, G. Sleijpen, and H. Van der Vorst. Jacobi-Davidson Style QR and QZ Algorithms for the Reduction of Matrix Pencils. SIAM J. Sci. Comput., 20(1):94-125, 1998. doi : $10.1137 /$ S1064827596300073.

[93] Xiaoye S. Li and James W. Demmel. SuperLU_DIST: A Scalable Distributed-memory Sparse Direct Solver for Unsymmetric Linear Systems. ACM Trans. Math. Softw., 29(2):110-140, June 2003. doi : 10.1145/779359.779361.

[94] C. L. Lawson, R. J. Hanson, D. R. Kincaid, and F. T. Krogh. Basic Linear Algebra Subprograms for Fortran Usage. ACM Trans. Math. Softw., 5(3):308-323, Sept 1979. doi : $10.1145 / 355841.355847$.

[95] J. J. Dongarra, Jeremy Du Croz, Sven Hammarling, and I. S. Duff. A Set of Level 3 Basic Linear Algebra Subprograms. ACM Trans. Math. Softw., 16(1):1-17, March 1990. doi : $10.1145 / 77626.79170$.

[96] Gregory R. Werner and John R. Cary. A stable FDTD algorithm for non-diagonal, anisotropic dielectrics. J. Comput. Phys., 226(1):1085 - 1101, 2007. doi:10.1016/ j.jcp. 2007.05 .008 .

[97] H. Voss. A Jacobi-Davidson method for nonlinear and nonsymmetric eigenproblems. Comput. Struct., 85(17-18):1284 - 1292, 2007. Computational Structures Technology. doi : $10.1016 /$ j . compstruc. 2006.08 .088 .

[98] Jin Au Kong. Electromagnetic Wave Theory. Wiley \& Sons, New York, 1986.

[99] M. L. Kales. Modes in Wave Guides Containing Ferrites. J. Appl. Phys., 24(5):604-608, 1953. doi : $10.1063 / 1.1721335$.

[100] G.C. Chinn, L.W. Epp, and G.M. Wilkins. Determination of the eigenfrequencies of a ferrite-filled cylindrical cavity resonator using the finite element method. IEEE Trans. Microw. Theory Techn., 43(5):1207-1209, 1995. doi : 10.1109/22 . 382089.

[101] A.A.Th.M Trier. Guided electromagnetic waves in anisotropic media. Appl. sci Res., Section B, 3(1):305-371, 1954. doi : 10.1007/BF02919911.

[102] W. R. Smythe. Reducing Ferrite Tuner Power Loss by Bias Field Rotation. IEEE Trans. Nucl. Sci., 30(4):2173-2175, Aug 1983. doi : 10.1109/TNS. 1983.4332751.

[103] M. Clemens and T. Weiland. Magnetic field simulation using conformal FIT formulations. IEEE Trans. Magn., 38(2):389-392, Mar 2002. doi : 10.1109/20.996104. 
Reports, conference proceedings and journal articles within these doctoral studies

[a] Klaus Klopfer, Wolfgang Ackermann, and Thomas Weiland. Eigenmode Computation for Ferrite-Loaded Cavity Resonators, GSI Scientific Report 2011, 2012.

[b] Klaus Klopfer, Wolfgang Ackermann, and Thomas Weiland. Eigenmode Computation for Ferrite-Loaded Cavity Resonators, Proceedings, 3rd International Particle Accelerator Conference (IPAC 2012), 2012. MOPPC058.

[c] Klaus Klopfer, Wolfgang Ackermann, and Thomas Weiland. Eigenmode Computation for Ferrite-Loaded Cavity Resonators, Proceedings, 11th International Computational Accelerator Physics Conference (ICAP 2012), 2012. THACC2.

[d] Klaus Klopfer, Wolfgang Ackermann, and Thomas Weiland. Eigenmode Computation for Ferrite-Loaded Cavity Resonators, GSI Scientific Report 2012, 2013.

[e] Klaus Klopfer, Wolfgang Ackermann, and Thomas Weiland. Eigenmode Computation for the GSI SIS 18 Ferrite Cavity, Proceedings, 4th International Particle Accelerator Conference (IPAC 2013), 2013. MOPWO006.

[f] Klaus Klopfer, Uwe Niedermayer, Harald Klingbeil, Wolfgang Ackermann, Hans Günther König, and Thomas Weiland. Measurement of the magnetic properties of the Ferroxcube $8 C 12$ material, GSI Scientific Report 2013, 2014.

[g] Klaus Klopfer, Uwe Niedermayer, Harald Klingbeil, Wolfgang Ackermann, Hans Günther König, and Thomas Weiland. Measurement of the magnetic material properties and simulation and modeling of ferrite-loaded cavities. Submitted for publication in Phys. Rev. ST Accel. Beams, 2014.

[h] Klaus Klopfer, Wolfgang Ackermann, and Thomas Weiland. Computation of Complex Eigenmodes for Resonators Filled With Gyrotropic Materials. Accepted for publication in IEEE Trans. Magn., 2014. doi:10.1109/TMAG. 2014.2338275. 


\section{Acknowledgments}

At the end of this thesis, I would like to express my gratitude to everyone who helped and supported me during my doctoral studies. Particularly, I would like to thank...

- GSI for financial support as well as the graduate school HGS-HIRe for the opportunity to participate in several lecture weeks and soft skill courses.

- Prof. Dr. Thomas Weiland for scientific supervision and the opportunity to work on an interesting topic at the institute, which provides excellent resources for an academic research.

- Prof. Dr. Harald Klingbeil for agreeing to act as second examiner. Moreover, I am deeply grateful for helpful discussions and support regarding the material measurements and related simulations.

- Prof. Dr. Herbert De Gersem for helpful advice regarding the magnetostatic solver.

- Prof. Dr. Oliver Boine-Frankenheim for enriching the PhD-committee meetings as external supervisor with his expertise.

- Dr. Wolfgang Ackermann for lots of helpful discussions and useful advice on a variety of subjects. I also acknowledge thorough reviewing of manuscripts for publications.

- Dr. Hans Günter König for providing support for the measurements at GSI.

- my colleague Uwe for valuable support and his never-ending enthusiasm for the measurements at GSI.

- my office colleagues for bearing nice company, in particular...

... Ulrich for always having the time to answer questions no matter how trivial or difficult the problem may look like.

... Cong for the great time we had on accelerator conferences all over the world, not to mention the conference dinners.

... Laura for occasionally joining the afternoon break in the park.

- the other members of the TEMF cinema organization team (Uli and in the beginning Laura) for helping to have a couple of great TEMF cinema evenings.

- my colleagues Jens and Vladimir for making the stay on the 2nd floor more enjoyable.

- my colleague Todorka for lots of conversations, no matter if they were about eigenvectors, grammar, applications or other things.

- Hai Van for sometimes bearing company in the kitchen at lunch time.

- Joel Alain and Konstantinos for occasionally joining the afternoon break.

- the "park group" for joining on the way back through the park after mensa (and dismissing the "coffee group").

- Heike from "K-Stone" for her kind support regarding administration.

- all other colleagues from TEMF who have been keeping a comfortable atmosphere and made us have an enjoyable time.

- my father and sisters, for their support during my studies and interest in what I have been working on. My heartfelt thanks for everything are addressed to you, dear Mama. 


\section{Curriculum Vitae}

The Curriculum Vitae is not included in this version for data protection. 